Les excitations dans la matière condensée 
Collection SFN 10 (2010) 3-136

(C) Owned by the authors, published by EDP Sciences, 2010

DOI: $10.1051 / \mathrm{sfn} / 2010001$

\title{
Les excitations dans la matière condensée : vibrations et phonons
}

\author{
H. Schober ${ }^{1,2}$ and S. Rols ${ }^{1}$ \\ ${ }^{1}$ Institut Laue Langevin, 6 rue Jules Horowitz, 38042 Grenoble Cedex 9, France \\ 2 I'Université Joseph Fourier, UFR de Physique, 38041 Grenoble Cedex 9, France
}

\begin{abstract}
Résumé. La fonctionnalité d'un matériau est fortement liée à sa capacité à subir des changements en réponse aux variations de l'environnement. Dans la plupart des cas, il suffit d'étudier la réponse du matériau au premier ordre, c'est-à-dire en régime linéaire. La réponse linéaire aux sollicitations extérieures est étroitement connectée aux fluctuations du système à l'état d'équilibre. Il est donc possible d'étudier les phénomènes thermodynamiques et même les phénomènes de transport par l'intermédiaire des fluctuations. Comme nous allons le montrer, la compréhension des phénomènes physiques passe par l'étude des fluctuations. Cette approche vaut pour la chaleur spécifique, la supraconductivité, en passant par l'expansion thermique, la conductivité thermique ou électrique ainsi que pour les transitions de phase. Dans la matière condensée, les fluctuations concernent à la fois les ions et les électrons. Nous allons montrer dans quelles conditions il est possible de découpler les deux systèmes (approximation adiabatique ou de BornOppenheimer) pour ensuite déterminer la nature des excitations ioniques dans un système dit harmonique. On introduira à cette occasion les concepts de phonon, fréquence et vecteur propre, ainsi que de surface de dispersion. Ayant établi la notion d'harmonicité, nous pourrons aborder les déviations du système par rapport à celle-ci. L'anharmonicité est responsable de phénomènes tels que l'expansion et la conductivité thermique. Nous allons conclure en revenant sur l'approximation adiabatique par l'intermédiaire du couplage des phonons aux électrons. Ce formalisme ouvrira la voie à la supraconductivité classique.
\end{abstract}

1 Motivation : la relation étroite entre fluctuations et fonctionnalité

2 Prélude : notions de bases $\quad 6$

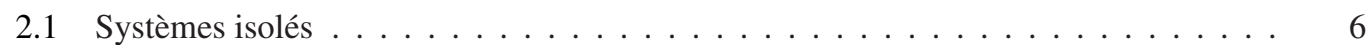

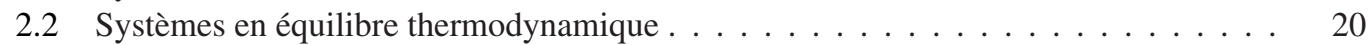

2.3 Occupation des niveaux à une particule . . . . . . . . . . . . . . . . . . 22

2.4 Fluctuations, corrélations et réponse linéaire . . . . . . . . . . . . . . . . 29

3 Dynamique des réseaux $\quad 41$

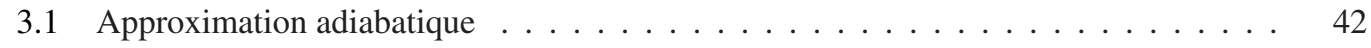

3.2 Approximation harmonique . . . . . . . . . . . . . . . . . . . 44

3.3 Interlude: chaîne linéaire . . . . . . . . . . . . . . . . . . . . . . . 45

3.4 La matrice dynamique dans l'espace direct . . . . . . . . . . . . . . . . . 68

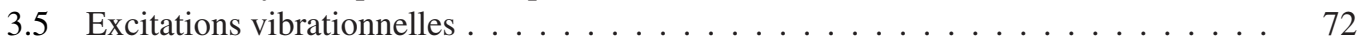

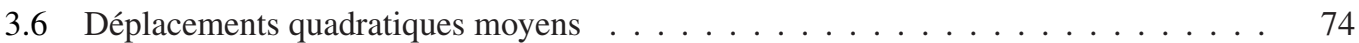

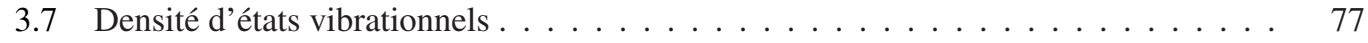

3.8 Fonctions thermodynamiques . . . . . . . . . . . . . . . . . . . 77

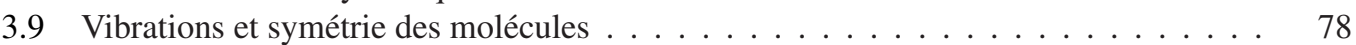

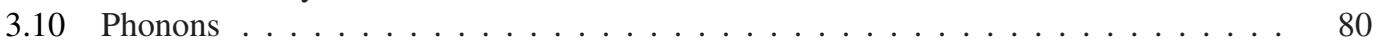

This is an Open Access article distributed under the terms of the Creative Commons Attribution-Noncommercial License 3.0, which permits unrestricted use, distribution, and reproduction in any noncommercial medium, provided the original work is properly cited. 
3.11 Localisation . . . . . . . . . . . . . . . . . . . . . . 92

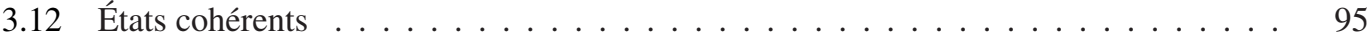

3.13 L'analogie avec les électrons de Bloch . . . . . . . . . . . . . . . . . . . . . . . . . . . . . . . . . . . . .

3.14 Calculs des éléments de la matrice dynamique . . . . . . . . . . . . . . . . . . . . . 97

3.15 Exemple concret : les phonons du quartz . . . . . . . . . . . . . . . . . . . . . . . . . 99

3.16 Exemple concret : la dynamique des fullerènes $\mathrm{C}_{60} \ldots \ldots \ldots$

4 Au-delà de l'approximation harmonique $\quad 107$

4.1 Expansion thermique et approximation quasi-harmonique . . . . . . . . . . . . 107

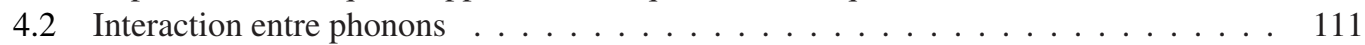

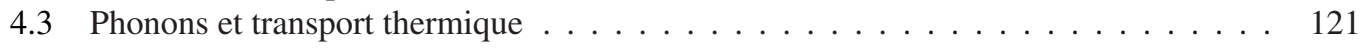

4.4 Couplage électron-phonon . . . . . . . . . . . . . . . . . . . . . 129

Références

\section{MOTIVATION : LA RELATION ÉTROITE ENTRE FLUCTUATIONS ET FONCTIONNALITÉ}

La propriété microscopique la plus remarquable d'un matériau est certainement sa structure atomique. Elle est décrite par les corrélations qui existent entre les positions des atomes moyennées dans le temps. Etant donné que la structure est le reflet direct des interactions entre atomes, elle permet souvent de prédire correctement certaines propriétés physiques d'un matériau. L'étude de la dynamique s'intéresse aux déviations des paramètres du système par rapport à la moyenne. Il est donc par définition impossible de s'attaquer à la dynamique sans une solide connaissance de la structure.

Néanmoins, la connaissance d'un matériau reste très incomplète sans l'apport de la dynamique. Les effets dynamiques deviennent, en général, de plus en plus importants avec l'augmentation de la température. Ainsi, elle mène à la transformation du matériau quand les fluctuations d'énergie atteignent le niveau de l'énergie de formation. Cette énergie dépend fortement des unités structurales qui constituent le système. Elle est par exemple beaucoup plus élevée pour des liaisons covalentes entre atomes que pour des liaisons de van der Waals entre molécules. Dans le cas d'unités structurales secondaires très complexes, qu'on rencontre par exemple en biologie, l'intervalle de stabilité peut être très petit et dépendre de manière cruciale de la présence des fluctuations elles-mêmes. Quand il s'agit du système des électrons ou des spins, l'échelle d'énergie est fixée par la température de mise en ordre de ceux-ci. Dans le cas de la supraconductivité classique, cette mise en ordre des électrons est induite par les fluctuations au sein du système des ions. Ce phénomène "d'état fondamental dynamique" est également rencontré dans les solides quantiques comme 1' ${ }^{4} \mathrm{He}$ superfluide.

Dans le détail, le rôle des fluctuations est beaucoup plus nuancé. Cela vaut en particulier pour les matériaux fonctionnels auxquels nous voudrions consacrer ici une attention toute particulière, étant donné que la mise au point de nouveaux dispositifs de haute technologie repose en grande partie sur l'exploitation de propriétés spécifiques des matériaux. Un matériau fonctionnel est très différent d'un matériau structural. Ses propriétés physiques et chimiques sont sensibles aux variations de l'environnement comme la température, la pression, les champs électromagnétiques, le $\mathrm{pH}$, la présence de molécules adsorbées, etc.... Parmi les matériaux fonctionnels classiques, on trouve les conducteurs thermiques, électriques et ioniques, les matériaux magnétiques, piezzo - et ferroélectriques, les luminescents et les photoréactifs. Cette liste se trouve constamment rallongée par des matériaux modernes comme les photochromes (qui changent de couleur en fonction de l'illumination), les thermochromes (qui changent de couleur avec la température), les multi-ferroïques ainsi que les matériaux pour l'optique non-linéaire.

La complexité de la fonctionnalité n'est pas la même dans tous les cas. La fonction d'un conducteur électrique consiste à assurer un transport de charge proportionnel à la tension appliquée. Le paramètre physique qui décrit cette fonctionnalité simple est la conductivité électrique ou son inverse, la résistance. 


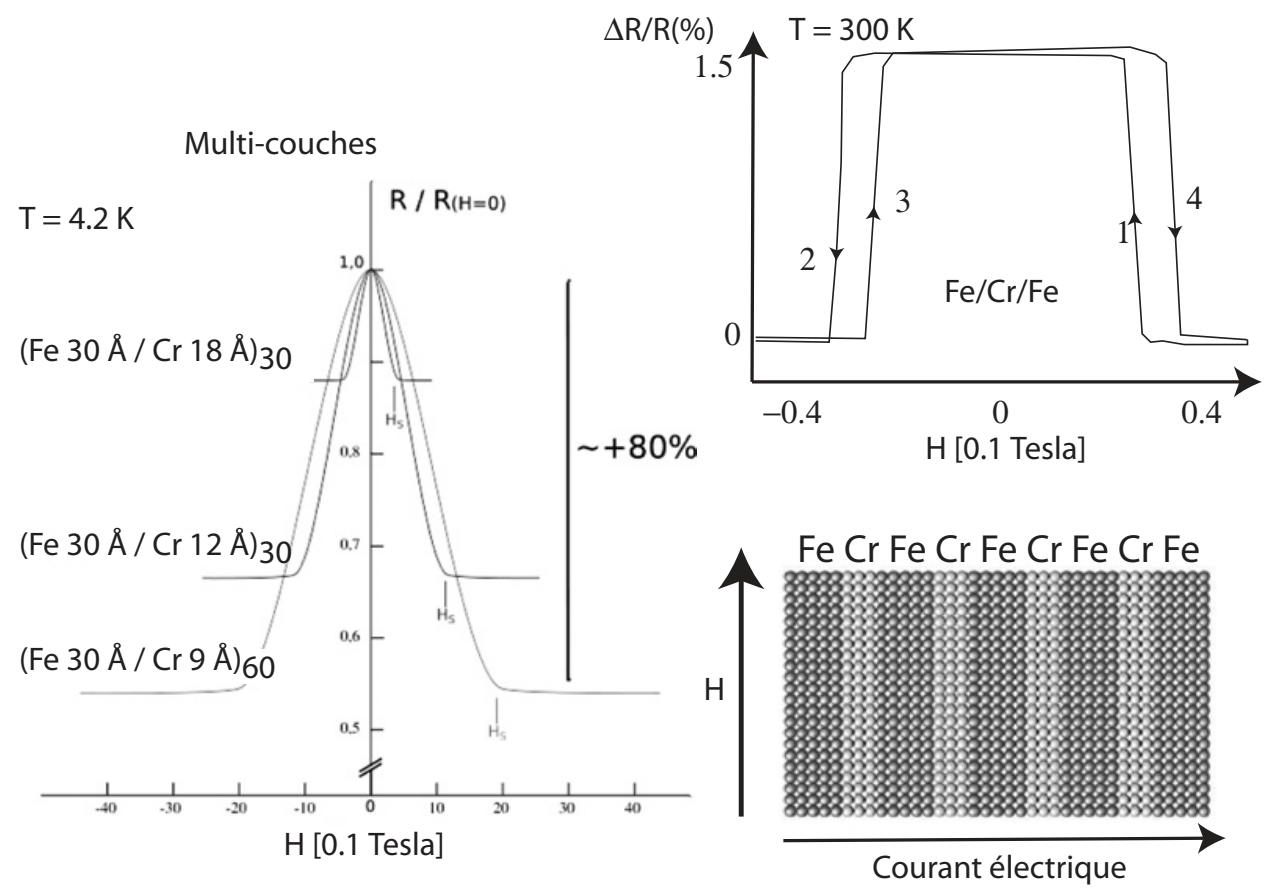

Figure 1. Magnétorésistance géante. Au moins deux couches ferromagnétiques sont séparées par un film ultramince (environ 1 nanomètre) de métal non ferromagnétique (par exemple, deux couches de fer séparées par du chrome : $\mathrm{Fe} / \mathrm{Cr} / \mathrm{Fe}$ ). Le champ magnétique est appliqué parallèlement aux couches et la résistance est mesurée perpendiculairement aux couches. Si les épaisseurs sont choisies correctement, les deux couches ferromagnétiques adjacentes présentent une aimantation antiparallèle à champ nul. Le champ magnétique externe induit un renversement d'aimantation: les aimantations respectives des deux couches s'alignent et la résistance de la multicouche décroît. Les deux diagrammes montrent schématiquement les résultats des groupes d' Albert Fert et de Peter Grünberg, pour lesquels ces deux chercheurs ont obtenu le prix Nobel de physique en 2007. Fert a travaillé avec des multi-couches alors que Grünberg a utilisé des films minces de trois couches $\mathrm{Fe} / \mathrm{Cr} / \mathrm{Fe}$ seulement. Dans le système de Fert, à $4.2 \mathrm{~K}$ la résistance électrique varie de plus de $80 \%$ avec le champ magnétique appliqué. A la température ambiante et avec trois couches, l'effet est toujours de quelques pourcents. Ainsi le champ magnétique permet de piloter activement la résistance de ces matériaux fonctionnels et, à l'inverse, de détecter des champs magnétiques. Ces dispositifs ont trouvé leur application dans les têtes de lecture de nos disques durs d'ordinateur.

Dans les applications de base, la résistance n'est pas pilotée et sa variation en fonction d'autres paramètres externes, comme par exemple la température, est plutôt considérée comme une nuisance qui complique le dimensionnement des circuits. Ce point de vue change dès que le contrôle de la résistance devient le but même de la fonctionnalité du composant, comme c'est le cas des têtes de lecture magnétiques dans nos disques durs d'ordinateur, et qui sont basées sur le principe de la magnétorésistance géante. En appliquant des champs magnétiques assez faibles, il est possible de réduire la résistance d'une multicouche magnétique proprement dimensionnée de plusieurs dizaines de pourcents à température ambiante (voir figure 1). Dans ce cas, comme dans tous les autres, où une ou plusieurs propriétés ne sont pas seulement sensibles mais peuvent directement être contrôlées par l'application d'une stimulation extérieure, on parle de matériaux actifs ou intelligents. Le plus haut dégré de complexité est atteint avec des systèmes biologiques qui assurent leur propre reproduction.

Le défi technologique réside dans l'obligation de passer des propriétés intrinsèques de la matière au matériau mis en forme et intégré dans un dispositif fonctionnel. Ainsi la partie sensible d'un bio-capteur doit produire un signal électrique détectable, suite à la présence de molécules biologiques spécifiques adsorbées sur sa surface. Une des difficultés principales de l'architecture fonctionnelle est la nécessité d'obtenir un contrôle à la fois facile (sensible) et stable. Ces propriétés sont toutes les deux liées à 
l'énergie nécessaire pour déclencher l'événement qu'on a l'intention de piloter. Un seuil énergétique élevé rendra le contrôle stable mais aussi très gourmand en amplitude de signal. Inversement, un seuil faible assurera certainement un pilotage à partir de signaux faibles mais malheureusement exposerait le dispositif au danger de variations spontanées provoquées par les fluctuations naturelles du système de contrôle. La situation est rendue encore plus complexe par le fait que les fluctuations elles-mêmes peuvent contribuer à la stabilisation du système par l'intermédiaire des forces entropiques. En résumé, stabilité et sensibilité sont des exigences a priori contradictoires. Elles doivent être judicieusement équilibrées pour optimiser la fonctionnalité. Ceci explique que des systèmes très complexes, avec des fonctionnalités très précises, ont souvent des plages de fonctionnement assez limitées. Ce fait est bien connu en biologie.

Il s'en suit que l'optimisation technologique d'un dispositif fonctionnel passe impérativement par la connaissance des fluctuations dans la matière qui le compose. Ceci relève de la physique de la matière condensée. Tout d'abord, l'étude des fluctuations à l'état d'équilibre va nous renseigner directement sur la réponse aux sollicitations extérieures, pourvu que nous nous limitions au régime linéaire. Il est même possible d'étudier les phénomènes de transport par l'intermédiaire des fluctuations. Etant donné que les dimensions des matériaux mis en œuvre sont de plus en plus petites, il est évident que les investigations doivent prendre en compte la structure microscopique de la matière, et donc que les fluctuations doivent être étudiées à l'échelle atomique. Cela nécessite une sonde sensible aux énergies mises en œuvre lors des fluctuations à l'échelle de l'Ångström. Les neutrons sont une sonde idéale pour ce genre d'investigation.

Nous voudrions terminer cette section par quelques nuances. Tous les systèmes pour lesquels nous étudierons les fluctuations seront des systèmes en équilibre thermodynamique. Les fonctions de réponses obtenues à partir des fluctuations correspondront aux réponses d'un système proche de son état d'équilibre. Par conséquent, le formalisme ne se prêtera pas à la description de systèmes métastables proches d'une instabilité. L'instabilité peut être déclenchée soit par une fluctuation interne, soit par un événement externe. Un exemple typique de telles instabilités est la cristallisation d'un amorphe ou d'un liquide surfondu, soit spontanée, soit induite par un germe. Beaucoup de mécanismes de contrôles technologiques impliquent des systèmes métastables. C'est le cas de toutes les vannes qui ont comme rôle le contrôle de réservoirs métastables. Les vannes de spins sont un exemple de vanne physique. Le contrôle de sous-ensembles métastables est même le fondement de tout organisme biologique.

\section{PRÉLUDE : NOTIONS DE BASES}

Dans cette partie, nous allons introduire quelques notions de base de la mécanique quantique, indispensables pour la suite. Il est particulièrement important de préciser la terminologie utilisée et de définir ce que nous entendons par excitation, fluctuation et dynamique d'un système. Dans cette perspective, nous commencerons par décrire un système quantique à l'échelle atomique, pour finir avec la réponse d'un système thermodynamique à une perturbation. Dans tous les cas, nous nous appuierons sur des exemples simples. Il est évident que la place allouée dans ce livre à cette introduction ne nous permettra que d'effleurer la surface de ce sujet très varié et scientifiquement fascinant que constitue la physique des solides. Le lecteur intéressé trouvera des informations exhaustives dans les textes classiques de la physique des solides comme par exemple dans le Ashcroft-Mermin [1], le Kittel [2] ou le livre plus récent de M.T. Dove [3].

\subsection{Systèmes isolés}

\subsubsection{Fonction d'onde et niveaux d'énergie d'une particule libre}

A l'échelle atomique, tout matériau est décrit par un opérateur de Hamilton $\mathbf{H} .{ }^{1}$ C'est l'opérateur de l'énergie. Il inclut l'énergie cinétique de toutes les particules ainsi que toutes les interactions entre

\footnotetext{
${ }^{1}$ Nous indiquerons les opérateurs quantiques soit par des caractères gras, soit par un accent circonflexe $(\mathbf{H}$ ou $\hat{H})$.
} 
elles. Si un système n'est pas soumis à des perturbations temporelles, son énergie est conservée. Par conséquent les états quantiques ${ }^{2}\left|\psi_{n}\right\rangle$ décrivant ce système doivent ${ }^{3}$ être des états propres de l'Hamiltonien $\mathbf{H}$

$$
\mathbf{H}\left|\psi_{n}\right\rangle=E_{n}\left|\psi_{n}\right\rangle .
$$

L'indice $n$ dénombre les états propres, également appelés stationnaires d'énergie $E_{n}$. Les fonctions propres ne sont pas des observables physiques. L'évolution d'une fonction d'onde stationnaire dans le temps est donnée par l'expression

$$
\left|\psi_{n}(t)\right\rangle=\left|\psi_{n}(t=0)\right\rangle e^{-i \frac{E_{n}}{\hbar} t} .
$$

Pour une particule libre ${ }^{4}$ l'opérateur de Hamilton correspond au seul terme d'énergie cinétique ${ }^{5}$

$$
\begin{gathered}
\mathbf{H}\left|\psi_{n}\right\rangle=\frac{p^{2}}{2 m}\left|\psi_{n}\right\rangle \\
\equiv-\frac{\hbar^{2}}{2 m}\left(\frac{\partial^{2}}{\partial x^{2}}+\frac{\partial^{2}}{\partial y^{2}}+\frac{\partial^{2}}{\partial z^{2}}\right) \psi_{n}(x, y, z) \equiv-\frac{\hbar^{2}}{2 m} \vec{\nabla} \cdot \vec{\nabla} \psi_{n}(\vec{r}) \equiv-\frac{\hbar^{2}}{2 m} \Delta \psi_{n}(\vec{r}) .
\end{gathered}
$$

Les solutions de l'équation 2.1 dans ce cas spécifique sont des ondes planes

$$
\psi_{\vec{k}}(\vec{r})=\frac{1}{\sqrt{V}} \exp (i \vec{k} \cdot \vec{r})=\frac{1}{\sqrt{V}}(\cos (\vec{k} \cdot \vec{r})+i \sin (\vec{k} \cdot \vec{r}))
$$

caractérisées par le vecteur d'onde $\vec{k}$. Le spectre en énergie est relié aux vecteurs d'onde par l'expression

$$
E(k)=\frac{\hbar^{2} k^{2}}{2 m} .
$$

Cette solution peut être directement transposée à un système de $N$ particules indépendantes, c'est-àdire à des particules qui n'interagissent pas les unes avec les autres. Chaque particule indépendante occupera un niveau d'énergie donné par l'éq. 2.5 correspondant à une fonction d'onde plane donnée par l'éq. 2.4. Pour des fermions, comme par exemple les électrons, cette occupation sera unique pour un spin donné. Pour des bosons, une occupation multiple est possible. La probabilité de rencontrer les particules $l=1 \ldots N$ dans les volumes $V_{l}$, quand le système se trouve dans l'état $\left|\psi_{n}\right\rangle$, est donnée par l'expression

$$
\int_{V_{1}} \mathrm{~d}^{3} r_{1} \ldots \int_{V_{N}} \mathrm{~d}^{3} r_{N} \psi \psi_{n}^{*}\left(\vec{r}_{1} \ldots \vec{r}_{N}\right) \psi_{n}\left(\vec{r}_{1} \ldots \vec{r}_{N}\right) .
$$

Dans l'équation 2.4, $V$ est donc le volume de normalisation pour des particules indépendantes. Il est important de distinguer la notion d'état du système de la notion de niveau occupé par une particule faisant partie du système.

En résolvant l'équation differentielle 2.1, nous avons implicitement supposé que le sytème est périodique, c'est-à-dire que le système remplit les conditions aux limites périodiques ${ }^{6}$

$$
\begin{aligned}
& \psi\left(x, y, z+L_{z}\right)=\psi(x, y, z), \\
& \psi\left(x, y+L_{y}, z\right)=\psi(x, y, z), \\
& \psi\left(x+L_{x}, y, z\right)=\psi(x, y, z),
\end{aligned}
$$

\footnotetext{
${ }^{2}$ Pour $N$ particules la fonction d'onde dépend de $N$ positions $\vec{r}_{i}, i=1 \ldots N$. En adoptant une écriture généralisée de la forme $\left\langle\psi_{n}\right\rangle \equiv \psi_{n}\left(\vec{r}_{1} \ldots \vec{r}_{N}\right)$, nous nous affranchissons de la représentation des fonctions d'ondes dans l'espace réel.

3 On trouvera des introductions très complètes à la mécanique quantique dans les livres dédiés à ce sujet, comme par exemple celui de C. Cohen-Tannoudji, B. Diu, et F. Lalö̈ [4].

4 Nous donnerons un exemple concret pour un système quantique en section 2.1.2, où nous présenterons l'oscillateur harmonique qui constitue la référence pour toute étude des excitations structurales.

5 Nous ne traiterons pas le spin explicitement dans ce chapitre.

6 Et de même pour la première dérivée de la fonction d'onde.
} 


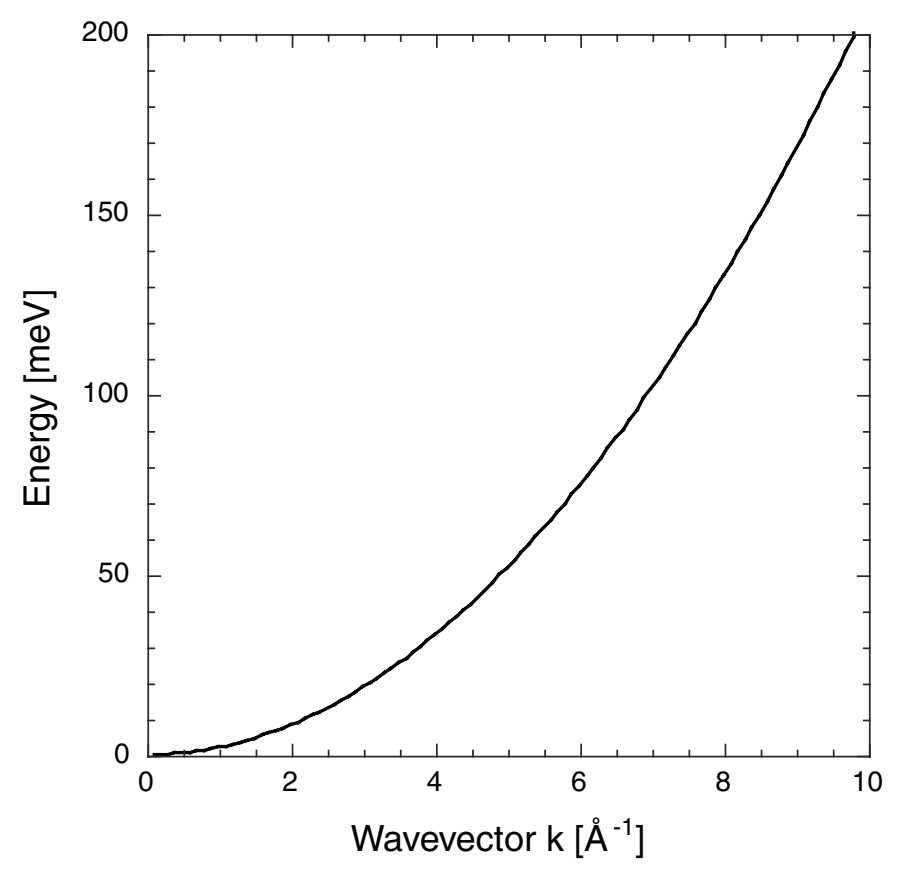

Figure 2. Spectre d'énergie pour une particule libre selon éq. 2.5. La masse $m$ a été choisie de sorte que le spectre corresponde à celui du neutron libre.

aussi appelées conditions aux limites de Born-Von Karman. ${ }^{7}$ Le volume de normalisation est donné par $V=L_{x} \cdot L_{y} \cdot L_{z}$. La probabilité de trouver une particule quelque part dans $V$ est donc égale à l'unité. La périodicité induit que les vecteurs d'onde ne peuvent prendre que les valeurs discrètes

$$
\vec{k}=\left(k_{x}, k_{y}, k_{z}\right)=\left(n_{x} \frac{2 \pi}{L_{x}}, n_{y} \frac{2 \pi}{L_{x}}, n_{z} \frac{2 \pi}{L_{x}}\right), \quad n_{x}, n_{y}, n_{z}=\ldots-2,-1,0,1,2, \ldots,
$$

donc dénombrables. Le fait que les états soient dénombrables est inhérent à la mécanique quantique et découle plus généralement de la nécessité de normaliser la fonction d'onde. Cette normalisation est requise pour toute particule réelle étant donné que la probabilité de détecter la particule selon l'éq. 2.6 doit être bien établie.

La densité des niveaux ${ }^{8}$, c'est-à-dire la densité des vecteurs $\vec{k}$, est donnée par l'expression

$$
\rho(\vec{k})=\frac{V}{8 \pi^{3}},
$$

ou en passant à la limite continue, et en profitant de l'isotropie d'une distribution dense des états $\vec{k}$

$$
g(k) d k=\frac{V}{8 \pi^{3}} 4 \pi k^{2} d k=\frac{V k^{2}}{2 \pi^{2}} d k .
$$

La fonction $g(k)$, appelée densité d'états, donne la probabilité de trouver un état dans une couche sphérique de rayon $\mathrm{k}$ et d'épaisseur dk dans l'espace des $\vec{k}$.

\footnotetext{
${ }^{7}$ Le choix des conditions est dicté purement par convenance mathématique. Il n'est donc pas unique. Il s'avérera néanmoins très adapté à l'étude des systèmes cristallins.

8 Ou encore le nombre de vecteurs $\vec{k}$ par unité de volume de l'espace des vecteurs d'onde.
} 
partie reélle

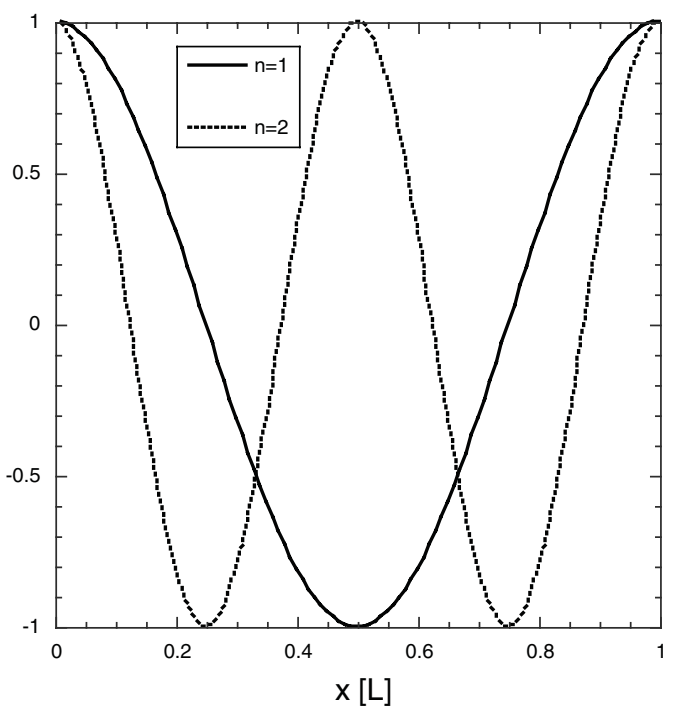

partie imaginaire

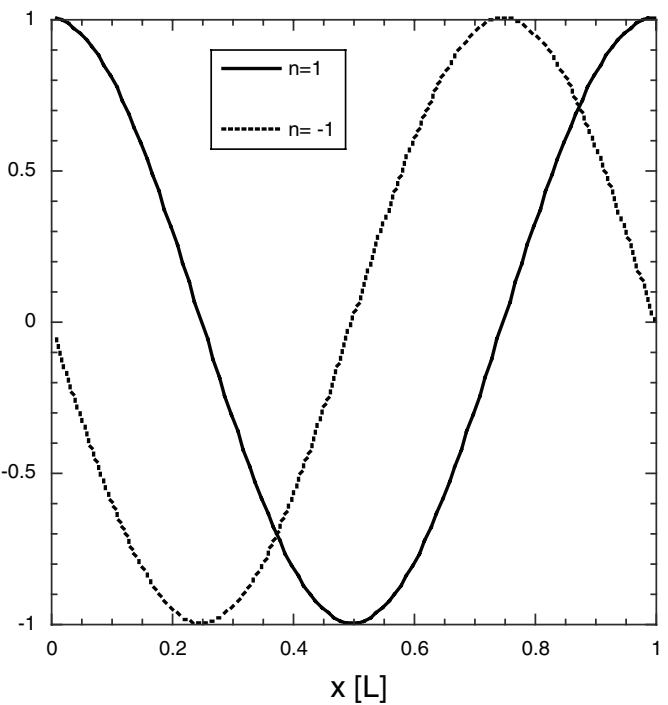

Figure 3. Exemples de fonctions d'onde d'une particule libre soumise à des conditions aux limites périodiques selon l'éq. 2.8 pour une dimension. Les fonctions d'onde sont intrinsèquement complexes. Les fonctions pour $n$ et $-n$ diffèrent dans leur partie imaginaire.

De même que les vecteurs $\vec{k}$, le spectre en énergie est discret et la différence entre niveaux est donnée par

$$
\Delta E=\frac{2 \pi^{2} \hbar^{2}}{m} \frac{1}{L^{2}}
$$

où, sans perte de généralité, nous avons choisi $L_{x}=L_{y}=L_{z}=L$.

Nous pouvons constater, à partir des équations 2.4 et 2.8 , que plusieurs fonctions d'onde $\psi_{\vec{k}}$ se partagent la même énergie $E_{n}$. Le niveau est dit dégénéré. Il est facile de démontrer que des fonctions propres partageant la même valeur propre peuvent être combinées linéairement pour donner d'autres fonctions propres. Ainsi les fonctions $\psi_{\text {paire }}(\vec{r})$ et $\psi_{\text {impaire }}(\vec{r})$ définies par

$$
\begin{gathered}
\psi_{\text {paire }}(\vec{r})=\frac{1}{\sqrt{2}}\left(\psi_{\vec{k}}(\vec{r})+\psi_{-\vec{k}}(\vec{r})\right)=\sqrt{\frac{2}{V}} \cos (\vec{k} \cdot \vec{r}), \\
\psi_{\text {impaire }}(\vec{r})=\frac{-i}{\sqrt{2}}\left(\psi_{\vec{k}}(\vec{r})-\psi_{-\vec{k}}(\vec{r})\right)=\sqrt{\frac{2}{V}} \sin (\vec{k} \cdot \vec{r})
\end{gathered}
$$

peuvent se substituer aux ondes planes $\psi_{\vec{k}}$ et $\psi_{-\vec{k}}$. Les fonctions de ce type, c'est-à-dire avec une parité bien définie, sont très utiles pour décrire les états dans des systèmes ayant une symétrie d'inversion. On notera que ces fonctions peuvent être choisies réelles.

\subsubsection{Particule confinée dans un puits harmonique}

Dans le cas des particules libres, il nous est possible de travailler avec un volume $V$ arbitrairement grand. Par conséquent les niveaux peuvent être rendus arbitrairement denses (éq. 2.9) et leur nature discrète pourrait n'être considerée que comme un problème académique. Par contre, dans un système dont les particules sont réellement confinées dans un volume $V$, comme par exemple des électrons du nuage atomique ou dans un puits quantique, le fait que les valeurs propres de l'Hamiltonien soient 
discrètes a des conséquences importantes et facilement observables. L'effet photoélectrique est un exemple particulièrement illustratif de ces effets de confinement sur le spectre des électrons. D'une manière plus générale, le caractère discret des niveaux est même primordial pour la compréhension de la physique des cristaux. Comme nous verrons dans les sections ultérieures, les états d'une particule dans un potentiel périodique peuvent être ramenés aux états de cette particule dans un potentiel confiné avec conditions aux limites périodiques. Le volume qui pilote la séparation des niveaux (pour un vecteur d'onde $\vec{k}$ donné) est dans ce cas celui de la maille primitive. On peut dire de manière générale que la distance énergétique entre niveaux augmente avec le confinement, c'est-à-dire qu'elle décroît avec le volume de la maille.

Le fait que les états soient discrets permet de les distinguer clairement. Nous pouvons ainsi parler de l'état de plus basse énergie, ou d'état fondamental. Les états d'énergie plus élevée sont appelés états excités. Le passage d'une particule d'un niveau bas à un niveau élevé se fait par excitation, l'inverse par désexcitation. Quelquefois le langage est imprécis et les états excités eux-mêmes sont appelés excitations.

Pour les vibrations, le potentiel le plus pertinent est le potentiel harmonique. Nous allons traiter ici de manière plus explicite le cas à une dimension. L'Hamiltonien s'écrit comme

$$
\mathbf{H}=\frac{\mathbf{p}^{2}}{2 m}+\frac{m \omega^{2}}{2} \mathbf{x}^{2}
$$

et en introduisant la constante

$$
\alpha=\sqrt{\left(\frac{m \omega}{\hbar}\right)}
$$

cette expression devient

$$
\mathbf{H}=\frac{\mathbf{p}^{2}}{2 m}+\frac{1}{2} \hbar \omega(\mathbf{x} \alpha)^{2} .
$$

Les valeurs propres sont données par

$$
E=\left(n+\frac{1}{2}\right) \hbar \omega, \quad n=0,1,2, \ldots
$$

Les fonctions propres $u_{n}(x)$ de l'oscillateur peuvent être déterminées exactement. Elle s'écrivent

$$
u_{n}(x)=\left(\frac{\alpha}{2^{n} n ! \sqrt{\pi}}\right) e^{-\frac{(\alpha x)^{2}}{2}} H_{n}(\alpha x)
$$

où les $H_{n}(\xi)$ représentent les fonctions d'Hermite

$$
\begin{gathered}
H_{0}(\xi)=1, \\
H_{1}(\xi)=2 \xi, \\
H_{2}(\xi)=4 \xi^{2}-2, \\
H_{3}(\xi)=8 \xi^{3}-12 \xi,
\end{gathered}
$$

Ces fonctions d'ondes $u_{n}(x)$ sont le produit d'une gaussienne de largeur $\alpha^{-1}$ et d'une fonction polynomiale d'ordre $n$. Elles possèdent donc une parité bien définie. Comme on peut le constater sur la figure 4, la particule se trouve centrée au milieu du puits harmonique dans son état fondamental $(n=0)$ et sa fonction d'onde possède une extension donnée par $\alpha^{-1}$. La longueur $\alpha^{-1}$ joue donc le rôle de longueur de confinement. Plus les flancs du puits sont raides, c'est-à-dire plus la fréquence $\omega$ est 
fonctions d'onde $u_{n}(x)$

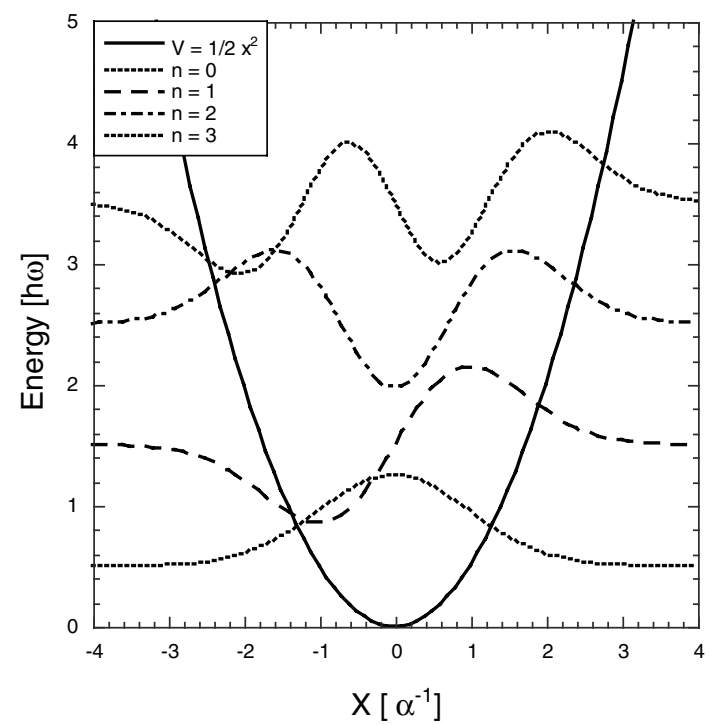

normes des fonctions d'onde $u_{n}(x) u_{n}{ }^{*}(x)$

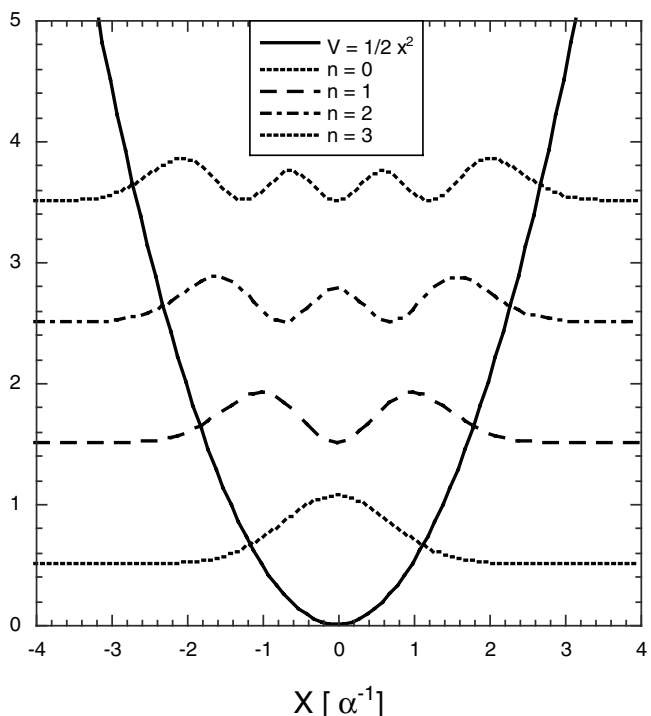

Figure 4. Fonctions d'onde et leurs normes pour une particule confinée dans un puits harmonique. La probabilité de trouver la particule se déplace du centre vers les bords de la parabole lorsque $n$ augmente. C'est le comportement attendu du point de vue de la mécanique classique : la particule passe plus de temps aux points de retournement (où sa vitesse s'annule) pendant une oscillation. Plus le potentiel est raide, plus les niveaux d'énergie sont espacés. La longueur de confinement intrinsèque du puits est donnée par l'expression $l_{\text {conf }}=2 \sqrt{2 \ln (2)} \alpha^{-1}=$ $2 \sqrt{2 \ln (2)} \sqrt{(\hbar / m \omega)}$.

grande, plus la particule est confinée. La distance entre niveaux, égale à $\hbar \omega$, s'agrandit. En d'autres termes, le confinement rend le spectre plus discret.

Il est utile pour la discussion des excitations du réseau de regarder de plus près la hiérarchie qui existe entre les niveaux de l'Hamiltonien harmonique. Dans cette optique nous reformulons le problème en introduisant de nouveaux opérateurs :

$$
\begin{aligned}
& \hat{\xi}=\alpha \mathbf{x}, \\
& \hat{\pi}=\frac{1}{\alpha \hbar} \mathbf{p},
\end{aligned}
$$

de sorte que

$$
\mathbf{H}=\frac{\hbar \omega}{2}\left(\hat{\xi}^{2}+\hat{\pi}^{2}\right)
$$

Nous introduisons

$$
\begin{aligned}
\hat{a} & =\frac{1}{\sqrt{2}}(\hat{\xi}+i \hat{\pi}), \\
\hat{a}^{+} & =\frac{1}{\sqrt{2}}(\hat{\xi}-i \hat{\pi}),
\end{aligned}
$$

et

$$
\hat{N}=\hat{a}^{+} \hat{a} .
$$


Pour faciliter la notation nous allons utiliser les symboles introduits par Dirac pour décrire un état de notre système :

$$
\begin{aligned}
|n\rangle & \equiv \psi_{n}(x) \\
\langle n| & \equiv \psi^{*}(x) \\
\left\langle n \mid n^{\prime}\right\rangle & =\int_{V} d x \psi(x) \psi^{*}(x) .
\end{aligned}
$$

On peut démontrer, soit de manière directe en faisant agir ces opérateurs sur les fonctions d'ondes $u_{n}(x)$, soit de manière générale en exploitant le fait que le commutateur

$$
\left[\hat{a}, \hat{a}^{+}\right]=1,
$$

que l'action de ces opérateurs sur les fonctions d'ondes de l'oscillateur harmonique est la suivante :

$$
\begin{aligned}
\hat{a}|n\rangle & =\sqrt{n}|n-1\rangle, \\
\hat{a}^{+}|n\rangle & =\sqrt{n+1}|n+1\rangle, \\
\hat{N}|n\rangle & =n|n\rangle .
\end{aligned}
$$

Nous constatons que l'opérateur $\hat{a}^{+}$fait passer l'état d'excitation du système du niveau $n$ au niveau $n+1$. On l'appelle ainsi opérateur de création. Son opérateur adjoint $\hat{a}$ abaisse l'état d'excitation et ramène le système du niveau $n+1$ au niveau $n$. On l'appelle donc opérateur d'annihilation. L'opérateur $\hat{N}$ donne le nombre $n$ d'excitations et s'appelle logiquement opérateur de nombre. Ensemble, $\hat{a}$ et $\hat{a}^{+}$ forment l'ensemble des opérateurs d'échelle.

En l'exprimant en fonction de ces opérateurs, l'Hamiltonien prend la forme très simple

$$
\mathbf{H}=\hbar \omega\left(\hat{N}+\frac{1}{2}\right) .
$$

L'équation de Schrödinger stationnaire s'écrit alors

$$
\mathbf{H}|n\rangle=\hbar \omega\left(\hat{N}+\frac{1}{2}\right)|n\rangle=\left(n+\frac{1}{2}\right) \hbar \omega|n\rangle,
$$

et tout état excité peut être construit à partir de l'état fondamental en remontant l'échelle

$$
|n\rangle=\frac{\left(\hat{a}^{+}\right)^{n}}{\sqrt{n !}}|0\rangle
$$

\subsubsection{Valeur moyenne d'une observable}

Toute observable physique $\mathcal{A}$ est reliée au système quantique par l'intermédiaire de la notion de valeur moyenne de l'opérateur A qui est associée à cette observable? .

Soit $\psi\left(\vec{r}_{1} \ldots \vec{r}_{N}, t\right)$ la fonction d'onde qui décrit l'état du système au temps t. La valeur moyenne d'une observable $\mathbf{A}$ au temps t est définie par

$$
\langle\mathbf{A}\rangle(t)=\int_{V_{1}} \mathrm{~d}^{3} r_{1} \ldots \int_{V_{N}} \mathrm{~d}^{3} r_{N} \psi^{*}\left(\vec{r}_{1} \ldots \vec{r}_{N}, t\right) \mathbf{A} \psi\left(\vec{r}_{1} \ldots \vec{r}_{N}, t\right) .
$$

\footnotetext{
${ }^{9}$ Nous parlons ici de valeur moyenne quantique qui donnerait la valeur de la mesure de l'observable $\mathcal{A}$ si tous les états stationnaires de l'opérateur A étaient équiprobables. La dissymétrie de la probabilité induite par la température modifie la valeur moyenne de $\langle\mathbf{A}\rangle(t)$ en fonction de la température du système et est traitée en physique statistique.
} 
Ainsi l'impulsion moyenne de la particule $l$ dans l'état $|\psi\rangle$ se calcule comme

$$
\left\langle\overrightarrow{\mathbf{p}}_{l}\right\rangle=\left\langle\psi\left|\overrightarrow{\mathbf{p}}_{l}\right| \psi\right\rangle=-i \hbar \int \mathrm{d}^{3} r_{1} \ldots \int \mathrm{d}^{3} r_{N} \psi^{*}\left(\vec{r}_{1} \ldots \vec{r}_{N}\right) \vec{\nabla}_{l} \psi\left(\vec{r}_{1} \ldots \vec{r}_{N}\right),
$$

ce qui donne pour les fonctions $\psi_{\vec{k}}(\vec{r})$

$$
\langle\overrightarrow{\mathbf{p}}\rangle=\hbar \vec{k}
$$

Pour les particules libres décrites par des fonctions d'onde $\psi_{\vec{k}}(\vec{r})$, les vecteurs d'onde peuvent donc être identifiés avec l'impulsion. Comme on le verra plus loin, ce n'est plus le cas pour des particules soumises à des potentiels périodiques. Ce n'est pas non plus correct pour les états $\psi_{\text {paire }}(\vec{r})$ et $\psi_{\text {impaire }}(\vec{r})$ (éq. 2.13). Il ne faut pas s'en étonner : une particule avec une impulsion finie change de direction sous l'effet de l'inversion.

Comme nous avons vu précédemment il est possible de décrire les états dans la base de l'espace de Hilbert, que constituent des fonctions propres d'une observable. En particulier

$$
|\Psi\rangle=\sum_{n}\left|\psi_{n}\right\rangle\left\langle\psi_{n} \mid \Psi\right\rangle=\sum_{n} c_{n}\left|\psi_{n}\right\rangle
$$

avec

$$
\mathbf{A}\left|\psi_{n}\right\rangle=a_{n}\left|\psi_{n}\right\rangle
$$

Les coefficients complexes du développement s'obtiennent à partir de la projection de la fonction $|\Psi\rangle$ sur les fonctions $\left|\psi_{n}\right\rangle$ à $t=0$. Ils sont donc fixés par les conditions initiales ${ }^{10}$. Formellement

$$
c_{n}=\left\langle\psi_{n}(t=0) \mid \Psi(t=0)\right\rangle=\int_{V_{1}} \mathrm{~d}^{3} r_{1} \ldots \int_{V_{N}} \mathrm{~d}^{3} r_{N} \psi_{n}^{*}\left(\vec{r}_{1} \ldots \vec{r}_{N}, t=0\right) \Psi\left(\vec{r}_{1} \ldots \vec{r}_{N}, t=0\right) .
$$

La forme 2.38 est l'expression la plus générale pour un état dynamique du système. Si les fonctions propres de l'opérateur A peuvent être choisies de sorte qu'elles soient aussi des fonctions propres de l'Hamiltonien, alors celles-ci sont des fonctions d'onde stationnaires dont nous connaissons bien la dépendance temporelle. C'est le cas si et seulement si cet opérateur A commute avec l'Hamiltonien $\mathbf{H}^{11}$. La valeur moyenne de A se calcule dans cette représentation stationnaire comme

$$
\langle\mathbf{A}\rangle(t)=\sum_{n, m} c_{m}^{*} c_{n}\left\langle\psi_{m}(t=0)|\mathbf{A}| \psi_{n}(t=0)\right\rangle e^{-i\left(\left(E_{n}-E_{m}\right) / \hbar\right) t}=\sum_{n} a_{n}\left|c_{n}\right|^{2} .
$$

Elle est donc indépendante du temps.

Un autre cas très intéressant est la valeur moyenne d'une observable quelconque quand le système se trouve dans un état stationnaire. La particule libre est un cas du genre. Partant de l'expression 2.35

\footnotetext{
${ }^{10}$ Dans un système classique, l'état initial est fixé par la connaissance au temps initial des positions et des vitesses de toutes les particules. Ces $6 N$ paramètres réels correspondent à $3 N$ coefficients complexes dans le système quantique. Nous donnerons un exemple concret dans la section dédiée aux paquets d'onde.

${ }^{11}$ La démonstration est aisée pour des valeurs propres non dégénerées : si l'opérateur $\mathbf{A}$ commute avec $\mathbf{H}$ alors on a

$$
[\mathbf{H}, \mathbf{A}]=\mathbf{H A}-\mathbf{A H}=0,
$$

et si $|\psi\rangle$ est une fonction propre de $\mathbf{H}$ alors

Il suit que

$$
\mathbf{H}|\psi\rangle=E|\psi\rangle .
$$

$$
\mathbf{H}(\mathbf{A}|\psi\rangle)=\mathbf{A} \mathbf{H}|\psi\rangle=E(\mathbf{A}|\psi\rangle) \text {. }
$$

La fonction $\mathbf{A}|\psi\rangle$ est donc une fonction propre de $\mathbf{H}$ avec valeur propre $E$. Etant donné que la valeur propre $E$ n'est pas dégénerée $\mathbf{A}|\psi\rangle$ doit être collinéaire à $|\psi\rangle$ et donc $\mathbf{A}|\psi\rangle=a|\psi\rangle$. La preuve est un peu plus complexe en cas de dégénerecence de $E$, mais on la trouve dans tous les livres de mécanique quantique.
} 
on obtient

$$
\begin{aligned}
\langle\mathbf{A}\rangle(t) & =\int_{V_{1}} \mathrm{~d}^{3} r_{1} \ldots \int_{V_{N}} \mathrm{~d}^{3} r_{N} e^{i(E / \hbar) t} \psi^{*}\left(\vec{r}_{1} \ldots \vec{r}_{N}, t=0\right) \mathbf{A} \psi\left(\vec{r}_{1} \ldots \vec{r}_{N}, t=0\right) e^{-i(E / \hbar) t} \\
& =\langle\mathbf{A}\rangle(t=0) .
\end{aligned}
$$

Pour un état stationnaire la valeur moyenne de toute observable physique est donc indépendante du temps. C'est cette propriété qui justifie l'adjectif stationnaire.

De façon générale, les fonctions d'onde stationnaires peuvent être classifiées selon les valeurs propres d'opérateurs quantiques A qui commutent avec l'Hamiltonien $\mathbf{H}$. Dans le cas où certaines valeurs propres sont dégénérées, la connaissance d'une valeur propre ne suffit pas à caractériser de façon unique l'état du système. On a alors besoin de connaître les valeurs propres associées à une (ou plusieurs) autre observable qui commute avec l'ensemble $(\mathbf{H}, \mathbf{A})$ pour caractériser l'état. Un ensemble complet d'observables qui commutent (ECOC) est un ensemble d'opérateurs qui commutent 2 à 2 et dont la connaissance des valeurs propres permet de caractériser l'état du système de façon univoque. Cet ECOC n'est en général pas unique : par exemple, les particules libres peuvent être caractérisées soit par leur moment cinétique $\vec{p}$, soit par leur moment angulaire $\left(\mathbf{l}^{2}, \mathbf{l}_{z}\right)$ par rapport à un point d'origine arbitraire. Un état du système est défini si toutes les valeurs propres de l'ECOC sont connues. Pour une particule libre dans l'ECOC de $\left(\mathbf{p}_{x}, \mathbf{p}_{y}, \mathbf{p}_{z}\right)$, ce sont les trois composantes de l'impulsion $\left(p_{x}, p_{y}, p_{z}\right)$ qui permettent de caractériser parfaitement un état. Pour l'électron de l'atome d'hydrogène, ce sont les nombres quantiques $(n, l, m)$ se référant aux opérateurs $\left(\mathbf{H}, \mathbf{l}^{2}, \mathbf{l}_{z}\right)$ qui permettent la caractérisation des états.

\subsubsection{Paquet d'onde}

Les fonctions d'onde trouvées en section 2.1.1 pour l'Hamiltonien d'une particule libre sont complètement délocalisées dans l'espace. Ceci n'est pas vraiment étonnant étant donné que ces fonctions sont des fonctions propres de l'opérateur d'impulsion et que cet opérateur ne commute pas avec l'opérateur position

$$
[\vec{r}, \vec{p}]=[\vec{r},-i \hbar \vec{\nabla}]=i \hbar
$$

L'opérateur position ne commute pas non plus avec l'Hamiltonien. La valeur moyenne de la position, si elle était bien définie, devrait donc changer avec le temps, ce qui est incompatible avec le caractère stationnaire des états à impulsion fixe. L'interprétation à donner à ces fonctions est celle d'un flux continu et normalisé de particules. Cette approche est identique à celle utilisée en optique ou en mécanique des ondes classiques. Si nous voulons décrire une particule plus ou moins localisée dans l'espace, nous devons recourir au concept de paquet d'onde, que nous traitons à une dimension pour simplifier. Selon l'expression 2.38 sa forme la plus générale est donnée par

$$
\psi(x, t)=\frac{1}{\sqrt{2 \pi}} \int w(k) e^{i(k x-\omega(k) t)} d k,
$$

où, pour une particule libre, la dispersion est donnée par

$$
w(k)=\frac{\hbar}{2 m} k^{2} .
$$

La fonction $w(k)$ décrit la distribution du paquet d'onde dans l'espace des vecteurs d'onde $\{k\}$ à l'instant $t=0$. La forme la plus appropriée pour cette fonction est une gaussienne

$$
w(k)=\left(\frac{1}{2 \pi(\Delta k)_{0}^{2}}\right)^{1 / 4} \exp \left[-i\left(k-k_{0}\right) x_{0}\right] \quad \exp \left[-\frac{\left(k-k_{o}\right)^{2}}{4(\Delta k)_{0}^{2}}\right]
$$



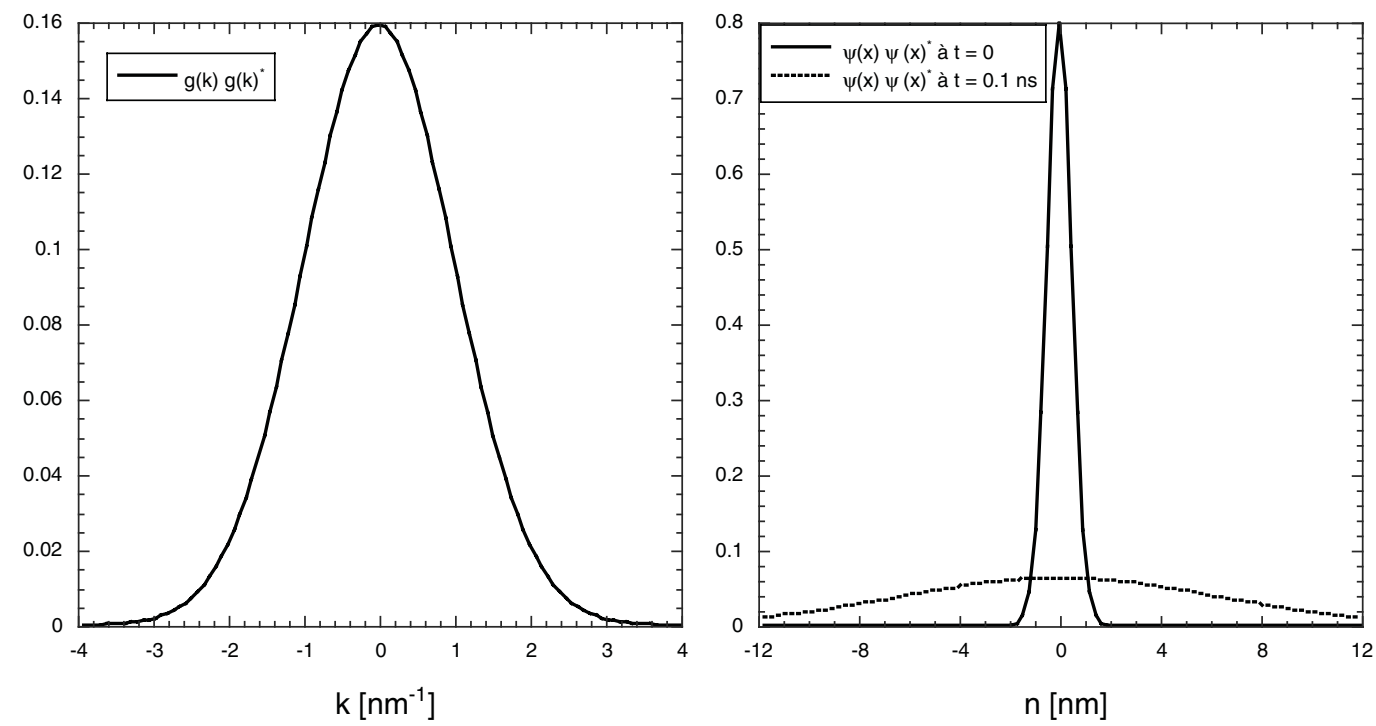

Figure 5. Paquet d'onde. La figure de gauche montre une distribution gaussienne en fonction du vecteur d'onde $k$. Cette distribution est stationnaire dans le temps. L'enveloppe de la fonction d'onde correspondante $\psi(x) \psi(x)^{*}$ est gaussienne et évolue avec le temps. La figure de droite montre l'étalement de l'enveloppe pour une particule de la masse d'un neutron avec la distribution $g(k)$ de gauche.

où le facteur

$$
\exp \left[-i\left(k-k_{0}\right) x_{0}\right]
$$

assure la phase correcte à $t=0$ et

$$
\left(\frac{1}{2 \pi(\Delta k)_{0}^{2}}\right)^{1 / 4}
$$

assure la normalisation. $x_{0}$ et $k_{0}$ représentent respectivement la position et le vecteur d'onde central à $t=0$. Si nous nous contentons d'un paquet au repos à l'origine $t=0$ nous pouvons mettre

$$
x_{0}=0, \quad k_{0}=0
$$

ce qui facilite considérablement la notation. Nous obtenons pour la fonction d'onde à $t=0$ une gaussienne de largeur

$$
(\Delta x)_{0}^{2}=\frac{1}{4} \frac{1}{(\Delta k)_{0}^{2}} .
$$

C'est la valeur minimale permise par la relation d'incertitude de Heisenberg

$$
\Delta x \cdot \Delta k \geq \frac{1}{2} .
$$

L'enveloppe $\psi(x, t) \psi(x, t)^{*}$ du paquet d'onde est une gaussienne. Si nous suivons l'évolution de cette enveloppe avec le temps, nous obtenons pour sa largeur

$$
(\Delta x)^{2}=(\Delta x)_{0}^{2}+\frac{\hbar^{2}}{4 m^{2}(\Delta x)_{0}^{2}} t^{2}=(\Delta x)_{0}^{2}+\frac{(\Delta p)_{0}^{2}}{m^{2}} t^{2}=(\Delta x)_{0}^{2}+(\Delta v)_{0}^{2} t^{2} .
$$


La fonction d'onde s'étale donc dans l'espace (voir figure 5) comme un ensemble de particules classiques avec une distribution de vitesse gaussienne de largeur $(\Delta v)_{0}$. Pour un neutron de masse $m=1.6749 \times 10^{-27} \mathrm{~kg}$ on obtient

$$
\frac{\hbar^{2}}{4 m^{2}}=9.9106 \cdot 10^{-16} \mathrm{~m}^{4} \mathrm{~s}^{-2}
$$

Donc, si le neutron a été confiné au début sur une échelle d'un nanomètre, il sera étalé sur près de 30 nanomètres après une nanoseconde.

On peut montrer que le maximum du paquet d'onde se déplace selon l'équation

$$
x_{m}=\left(\frac{\partial \omega}{\partial k}\right)_{k_{0}}=\frac{\hbar k_{0}}{m} t .
$$

C'est le résultat bien connu de la mécanique des ondes classiques selon lequel un paquet d'onde possède la vitesse de groupe

$$
\vec{v}_{g}=\frac{1}{\hbar}\left(\frac{\partial E(\vec{k})}{\partial \vec{k}}\right)_{\vec{k}_{0}}=\frac{1}{\hbar}\left(\frac{\partial E(\vec{k})}{\partial k_{x}}, \frac{\partial E(\vec{k})}{\partial k_{y}}, \frac{\partial E(\vec{k})}{\partial k_{z}}\right)_{\vec{k}_{0}}
$$

$E(\vec{k})$ décrit la dépendance de l'énergie en fonction de $\vec{k}$. Dans le cas d'une particule libre $v_{g}=\hbar k_{0} / m$. Le concept de paquet d'onde est très précieux dans la physique des solides partout où il faut décrire des processus de transport. Il nous permet de traiter dans beaucoup de cas de manière classique le mouvement entre collisions, c'est-à-dire que nous pouvons décrire les trajectoires dans des champs électromagnétiques externes ${ }^{12}$ en utilisant les équations du mouvement

$$
\frac{\partial \vec{r}}{\partial t}=\vec{v}_{g}(\vec{k})=\frac{1}{\hbar}\left(\frac{\partial E(\vec{k})}{\partial \vec{k}}\right)
$$

et

$$
\frac{\hbar \partial \vec{k}}{\partial t}=-e\left[\vec{E}(\vec{r}, t)+\frac{1}{c} \vec{v}_{g} \times \vec{H}(\vec{r}, t)\right]
$$

\subsubsection{Couplage et modes normaux}

Un des problèmes fondamentaux de la physique est le couplage entre les particules d'un système. Ce couplage par interaction est inhérent au système. Il faut le distinguer du couplage thermodynamique du système dans son intégralité à son environnement qui fera l'objet de la section suivante. Nous nous appliquerons ici à donner un exemple simple pour le couplage des particules par interaction directe. ${ }^{13}$ Cela nous permettra d'introduire le concept de modes normaux, qui plus tard sera essentiel pour décrire la dynamique du réseau.

Soient $x_{1}$ et $x_{2}$ les positions de deux atomes de masse $m$ assujetis à bouger dans une direction. Les atomes sont couplés aux murs et entre eux par l'intermédiaire de potentiels harmoniques, qui classiquement peuvent être décrits par des ressorts (voir figure 6). L'Hamiltonien de ce système s'écrit

$$
\mathbf{H}=\frac{\mathbf{p}_{1}^{2}}{2 m}+\frac{\mathbf{p}_{2}^{2}}{2 m}+\frac{1}{2} m \omega_{0}\left[\mathbf{x}_{1}^{2}+\mathbf{x}_{2}^{2}+\left(\mathbf{x}_{1}-\mathbf{x}_{2}\right)^{2}\right] .
$$

\footnotetext{
12 Ces champs doivent varier peu à l'échelle de l'extension du paquet d'onde. Le paquet d'onde lui-même doit être plus étendu que la maille cristalline.

13 Une présentation très pédagogique est donnée dans l'article de Johnson et Gutierrez [10].
} 

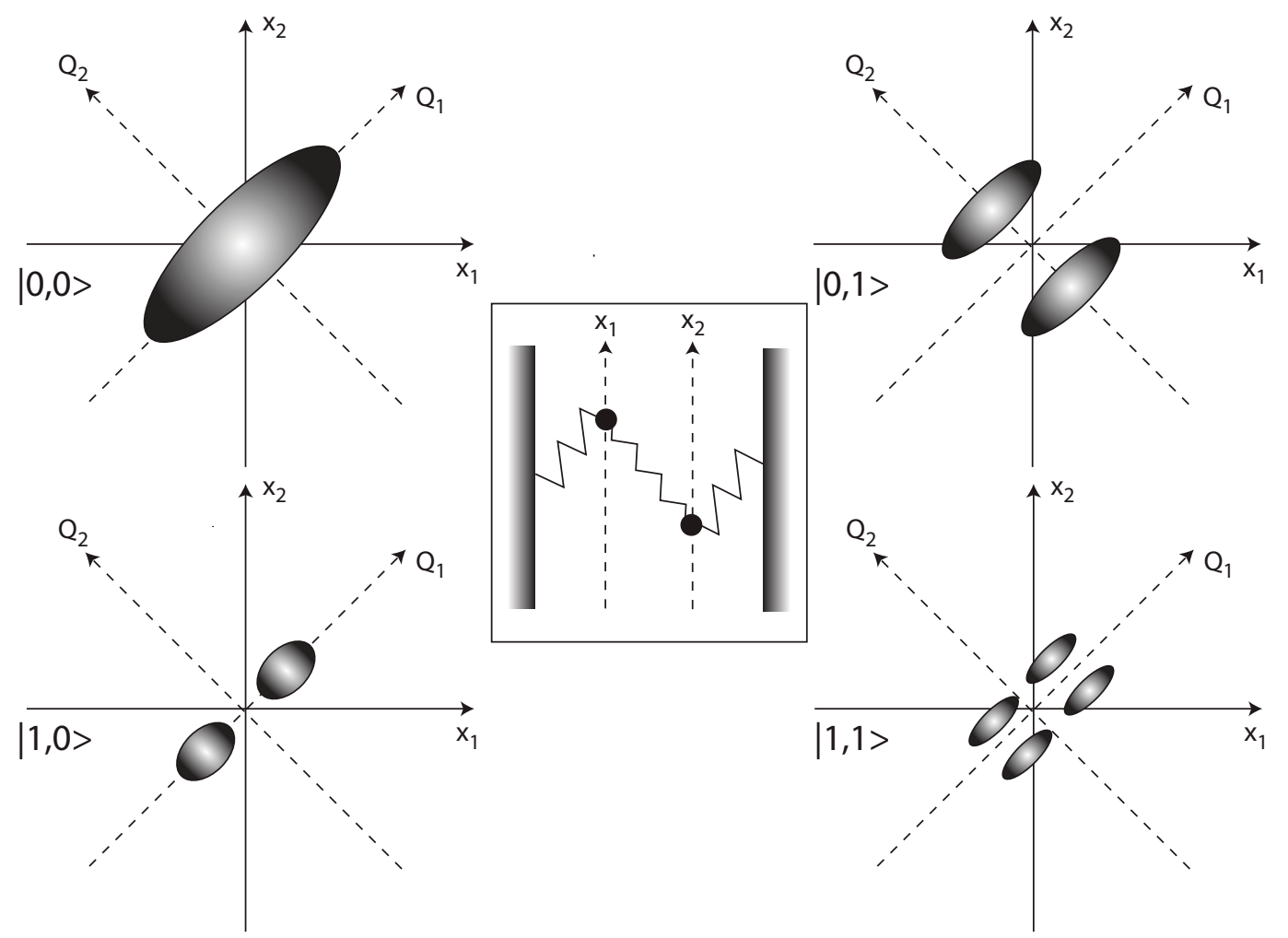

Figure 6. Probabilité de rencontrer les deux particules couplées entre elles et aux murs par une force harmonique. Le mouvement est limité à la direction $x$. $Q_{1}$ est la coordonnée normale du mouvement du centre de gravité, $Q_{2}$ est la coordonnée normale du mouvement relatif. Dans l'état fondamental la distribution est gaussienne avec une largeur qui est 3 fois plus important le long de $Q_{1}$. Pour les états excités, on reconnaît la périodicité des fonctions de Hermite 2.19, si on reste dans la base des $\left(Q_{1}, Q_{2}\right)$. Dans les coordonnées d'origine $\left(x_{1}, x_{2}\right)$, les fonctions n'ont plus de ressemblance avec les fonctions propres de l'oscillateur harmonique.

A cause du terme de couplage $\left(\mathbf{x}_{1}-\mathbf{x}_{2}\right)^{2}$, il ne peut pas s'écrire comme la somme de deux Hamiltoniens indépendants. Nous effectuons un changement de coordonnées en introduisant

$$
\begin{aligned}
& \mathbf{Q}_{1}=\frac{1}{\sqrt{2}}\left(\mathbf{x}_{1}+\mathbf{x}_{2}\right), \\
& \mathbf{Q}_{2}=\frac{1}{\sqrt{2}}\left(\mathbf{x}_{1}-\mathbf{x}_{2}\right),
\end{aligned}
$$

ainsi que

$$
\begin{aligned}
& \mathbf{P}_{1}=\frac{1}{\sqrt{2}}\left(\mathbf{p}_{1}+\mathbf{p}_{2}\right), \\
& \mathbf{P}_{2}=\frac{1}{\sqrt{2}}\left(\mathbf{p}_{1}-\mathbf{p}_{2}\right) .
\end{aligned}
$$

Ce changement de coordonnées peut aussi s'ecrire sous la forme plus compacte

$$
\left(\mathbf{Q}_{1}, \mathbf{Q}_{2}\right)=\tilde{S}\left(\begin{array}{l}
\mathbf{x}_{1} \\
\mathbf{x}_{2}
\end{array}\right),
$$




$$
\left(\mathbf{P}_{1}, \mathbf{P}_{2}\right)=\tilde{S}\left(\begin{array}{l}
\mathbf{p}_{1} \\
\mathbf{p}_{2}
\end{array}\right)
$$

avec la matrice de transformation

$$
\tilde{S}=\frac{1}{2}\left(\begin{array}{cc}
\sqrt{2}, & \sqrt{2} \\
\sqrt{2}, & -\sqrt{2}
\end{array}\right) .
$$

Il est facile de vérifier que l'Hamiltonien 2.61 se transforme en

$$
\mathbf{H}=\frac{\mathbf{P}_{1}^{2}}{2 m}+\frac{\mathbf{P}_{2}^{2}}{2 m}+\frac{1}{2} m \omega_{1}^{2} \mathbf{Q}_{1}^{2}+\frac{1}{2} m \omega_{2}^{2} \mathbf{Q}_{2}^{2},
$$

avec

$$
\begin{aligned}
& \omega_{1}=\omega_{0}, \\
& \omega_{2}=3 \omega_{0} .
\end{aligned}
$$

L'Hamiltonien 2.69 décrit deux oscillateurs indépendants dans les variables $\mathbf{Q}_{1}$ et $\mathbf{Q}_{2}$. Se réferrant à la mécanique classique, on se rend tout de suite compte que $\mathbf{Q}_{1}$ est l'opérateur qui correspond à la coordonnée du mouvement du centre de masse et $\mathbf{Q}_{2}$ l'opérateur qui correspond à la coordonnée du mouvement relatif entre les particules. On appelle par conséquent ces opérateurs, opérateurs des modes normaux. La matrice $\tilde{S}$ est orthogonale, c'est-à-dire ses vecteurs colonnes sont orthogonaux entre eux et de norme 1. Pour une matrice orthogonale la matrice inverse existe et est égale à sa transposée

$$
\tilde{S}^{-1}=\tilde{S}^{t} .
$$

On peut donc facilement invertir les expressions et obtenir les coordonnées originales à partir des coordonnées normales

$$
\begin{aligned}
& \left(\mathbf{x}_{1}, \mathbf{x}_{2}\right)=\tilde{S}^{t}\left(\begin{array}{l}
\mathbf{Q}_{1} \\
\mathbf{Q}_{2}
\end{array}\right), \\
& \left(\mathbf{p}_{1}, \mathbf{p}_{2}\right)=\tilde{S}^{t}\left(\begin{array}{l}
\mathbf{P}_{1} \\
\mathbf{P}_{2}
\end{array}\right) .
\end{aligned}
$$

Les fonctions d'ondes du système couplé sont de simples produits de fonction à une variable dans l'espace des modes normaux. Il peuvent s'exprimer comme

$$
\psi\left(Q_{1}, Q_{2}\right)=\sum_{m, n} c_{m n} \psi_{m}\left(Q_{1}\right) \psi_{n}\left(Q_{2}\right),
$$

dans la base des fonctions propres de l'Hamiltonien, avec

$$
\begin{aligned}
& {\left[\frac{\mathbf{P}_{1}^{2}}{2 m}+\frac{1}{2} m \omega_{1}^{2} \mathbf{Q}_{1}^{2}\right] \psi_{m}\left(Q_{1}\right)=\hbar \omega_{1}\left(m+\frac{1}{2}\right) \psi_{m}\left(Q_{1}\right),} \\
& {\left[\frac{\mathbf{P}_{2}^{2}}{2 m}+\frac{1}{2} m \omega_{2}^{2} \mathbf{Q}_{1}^{2}\right] \psi_{n}\left(Q_{2}\right)=\hbar \omega_{2}\left(n+\frac{1}{2}\right) \psi_{n}\left(Q_{2}\right) .}
\end{aligned}
$$

En particulier l'état fondamental est donné par

$$
\psi_{0}\left(Q_{1}, Q_{2}\right)=\psi_{0}\left(Q_{1}\right) \psi_{0}\left(Q_{2}\right)
$$


avec

$$
\begin{aligned}
& \psi_{0}\left(Q_{1}\right)=\left(\frac{\alpha}{\sqrt{\pi}}\right) e^{-\frac{\left(\alpha Q_{1}\right)^{2}}{2}}, \\
& \psi_{0}\left(Q_{1}\right)=\left(\frac{\alpha^{\prime}}{\sqrt{\pi}}\right) e^{-\frac{\left(\alpha^{\prime} Q_{2}\right)^{2}}{2}},
\end{aligned}
$$

et

$$
\begin{gathered}
\alpha=\sqrt{\left(\frac{m \omega_{0}}{\hbar}\right)}, \\
\alpha^{\prime}=\sqrt{\left(\frac{3 m \omega_{0}}{\hbar}\right)} .
\end{gathered}
$$

Les probabilités de présence des particules sont étalées selon les deux coordonnées normales. La distribution pour le centre de masse est 3 fois plus large que celle pour le mouvement relatif. Ce résultat confirme l'intuition selon laquelle le potentiel pour le mouvement du centre de masse est moins raide.

On retrouve toute la puissance des opérateurs d'échelle introduits dans la section 2.1.2 quand il s'agit de calculer les états excités des deux oscillateurs couplés. Par analogie avec les expressions 2.23, on définit

$$
\begin{array}{ll}
\hat{a}_{i}=\frac{1}{\sqrt{2}}\left(\hat{\xi}_{i}+i \hat{\pi}_{i}\right), & i=1,2, \\
\hat{a}_{i}^{+}=\frac{1}{\sqrt{2}}\left(\hat{\xi}_{i}-i \hat{\pi}_{i}\right), \quad i=1,2,
\end{array}
$$

avec

$$
\begin{aligned}
\hat{\xi}_{i} & =\alpha_{i} \mathbf{Q}_{i}, \\
\hat{\pi}_{i} & =\frac{1}{\alpha_{i} \hbar} \mathbf{P}_{i} .
\end{aligned}
$$

La fonction d'onde 2.79 des états excités se détermine à partir de la fonction de l'état fondamental en appliquant les deux opérateurs de création.

$$
\left|\psi_{m n}\right\rangle \equiv|m, n\rangle=\frac{\left(\hat{a}_{1}^{+}\right)^{m}}{\sqrt{m !}} \frac{\left(\hat{a}_{2}^{+}\right)^{n}}{\sqrt{n !}}|0,0\rangle .
$$

On va donc pouvoir décrire les états excités dans la représentation des modes normaux à l'aide des fonctions

$$
u_{n}\left(Q_{i}\right)=\left(\frac{\alpha_{i}}{2^{n} n ! \sqrt{\pi}}\right) e^{-\frac{\left(\alpha_{i} Q_{i}\right)^{2}}{2}} H_{n}\left(\alpha_{i} Q_{i}\right) .
$$

Mais attention, prises comme fonctions des positions des particules $\left(x_{1}, x_{2}\right)$, les fonctions d'onde n'ont plus grande chose à voir avec les fonctions d'onde de l'oscillateur harmonique (voir figure 6).

On peut démontrer mathématiquement que n'importe quel système qui ne comporte que des couplages du deuxième ordre dans les variables de position, peut être décomposé en modes normaux. En particulier, il est possible de caractériser les vibrations des molécules selon leurs modes normaux. En exemple nous montrons les modes normaux de vibration des molécules $\mathrm{CO}_{2}$ et $\mathrm{H}_{2} \mathrm{O}$ sur la figure 7 . Etant donné que des molécules isolées ne sont pas retenues par des forces dans l'espace (contrairement aux deux particules dans l'exemple précédant, les 6 modes normaux ${ }^{14}$ qui déplacent ou font tourner

143 translations selon $x, y$ et $z$ plus 3 rotations autour d'axes indépendants. 
la molécule dans son ensemble sans solliciter les liaisons entre les atomes ont des fréquences nulles. La fonction d'onde qui y correspond est uniforme dans tout l'espace, ce qui pose le problème de la normalisation, comme nous l'avons déjà vu précédemment. En général on peut négliger ces modes dits externes et se concentrer sur les modes dits internes de la molécule qui sont au nombre de $3 N-6$ et qui mettent en jeu des déformations de liaisons ( $N$ étant le nombre d'atomes composant la molécule).

\subsection{Systèmes en équilibre thermodynamique}

Dans la pratique, les systèmes qu'on étudie ne peuvent pas être considérés isolés de leur environnement. Même dans le cas extrême d'un atome seul dans l'espace, les fluctuations du vide du champ électromagnétique feront en sorte qu'un état excité $\left(E_{n}>E_{0}\right)$ n'aura pas une durée de vie infinie, et ne sera donc pas vraiment stationnaire. Du point de vue conceptuel, l'exemple le plus parlant est celui d'un système couplé à un bain thermique. ${ }^{15}$ Ce couplage laissera le système en équilibre thermique à une température $T$ permettant, soit le seul échange d'énergie (ensemble canonique) soit l'échange d'énergie et de particules (ensemble grand-canonique) avec le bain. Le fait que le système puisse échanger de l'énergie avec le réservoir implique que son énergie est amenée à fluctuer. Dans un ensemble canonique, le poids statistique d'un état stationnaire $\left|\psi_{n}\right\rangle$ d'énergie $E_{n}$ est donné par le facteur de MaxwellBoltzmann ${ }^{16}$

$$
p_{n}=\frac{1}{Z} \exp \left(-\frac{E_{n}}{k_{\mathrm{B}} T}\right)
$$

avec la fonction de partition canonique $Z$ définie par

$$
Z=\sum_{n} \exp \left(-\frac{E_{n}}{k_{\mathrm{B}} T}\right)=\sum_{n}\left\langle n\left|\exp \left(-\frac{\mathbf{H}}{k_{\mathrm{B}} T}\right)\right| n\right\rangle=\operatorname{Tr}\left[\exp \left(-\frac{\mathbf{H}}{k_{\mathrm{B}} T}\right)\right]
$$

et la constante de Boltzmann

$$
k_{\mathrm{B}}=0.08617 \mathrm{meV} / \mathrm{K} \quad \text { ou } \quad k_{\mathrm{B}}^{-1}=11.60 \mathrm{~K} / \mathrm{meV} .
$$

La somme dans l'équation 2.94 court sur tous les états possibles du système. La fonction de partition canonique $Z$ dépend directement de la température $T$ et indirectement du volume $V$ (par l'intermédiaire

\footnotetext{
15 On ne pourra ici que donner quelques idées sur la thermodynamique des solides. Le lecteur intéressé trouvera des informations plus vastes dans les livres. De bonnes introductions se trouvent par exemple dans Kittel et Kroemer [6] ou dans Blundell et Blundell [5].

16 Il n'est certainement pas exagéré d'affirmer que l'ensemble de la mécanique statistique est fondée sur ce résultat. Il peut être instructif de se rappeler l'origine du facteur de Maxwell-Boltzmann. Prenons un système $S$ (par exemple un oscillateur harmonique) couplé à un réservoir $R$ de manière à ce que le système puisse échanger de l'énergie avec le réservoir. Soit $U$ l'énergie de l'ensemble. Supposons que $S$ puisse se trouver dans des états $i$ et $j$ d'énergies respectives $E_{i}$ et $E_{j}$. Dans ce cas, la probabilité de trouver le système $S$ dans l'état $i$ est gouvernée par le nombre d'états du réservoir compatibles avec l'énergie $U-E_{i}$ (le raisonnement est identique pour l'état $j$ ). On obtient alors la relation
}

$$
\frac{P\left(E_{i}\right)}{P\left(E_{j}\right)}=\frac{\text { Nombre d'états accessibles au réservoir pour l'énergie } \quad\left(U-E_{i}\right)}{\text { Nombre d'états accessibles au réservoir pour l'énergie } \quad\left(U-E_{j}\right)}=\frac{\exp \left(S_{R}\left(U-E_{i}\right) / k_{\mathrm{B}}\right)}{\exp \left(S_{R}\left(U-E_{j}\right) / k_{\mathrm{B}}\right)}
$$

où nous avons introduit l'entropie du réservoir $S_{R}$ par l'intermédiaire du logarithme naturel de la dégénérescence des états. $E_{i}$ va toujours être très petite par rapport à $U$. En développant l'entropie en série et en se limitant au premier ordre en $E_{i}$ on obtient

$$
S_{R}\left(U-E_{i}\right)=S_{R}(U)-E_{i}\left(\frac{\partial S}{\partial U}\right)_{N, V}
$$

et en identifiant $(\partial S / \partial U)_{N, V}$ avec l'inverse de la température $T$ on obtient

$$
\frac{P\left(E_{i}\right)}{P\left(E_{j}\right)}=\frac{\exp -\left(E_{i} / k_{\mathrm{B}} T\right)}{\exp -\left(E_{j} / k_{\mathrm{B}} T\right)}
$$

ce qui mène directement à l'équation 2.93. 

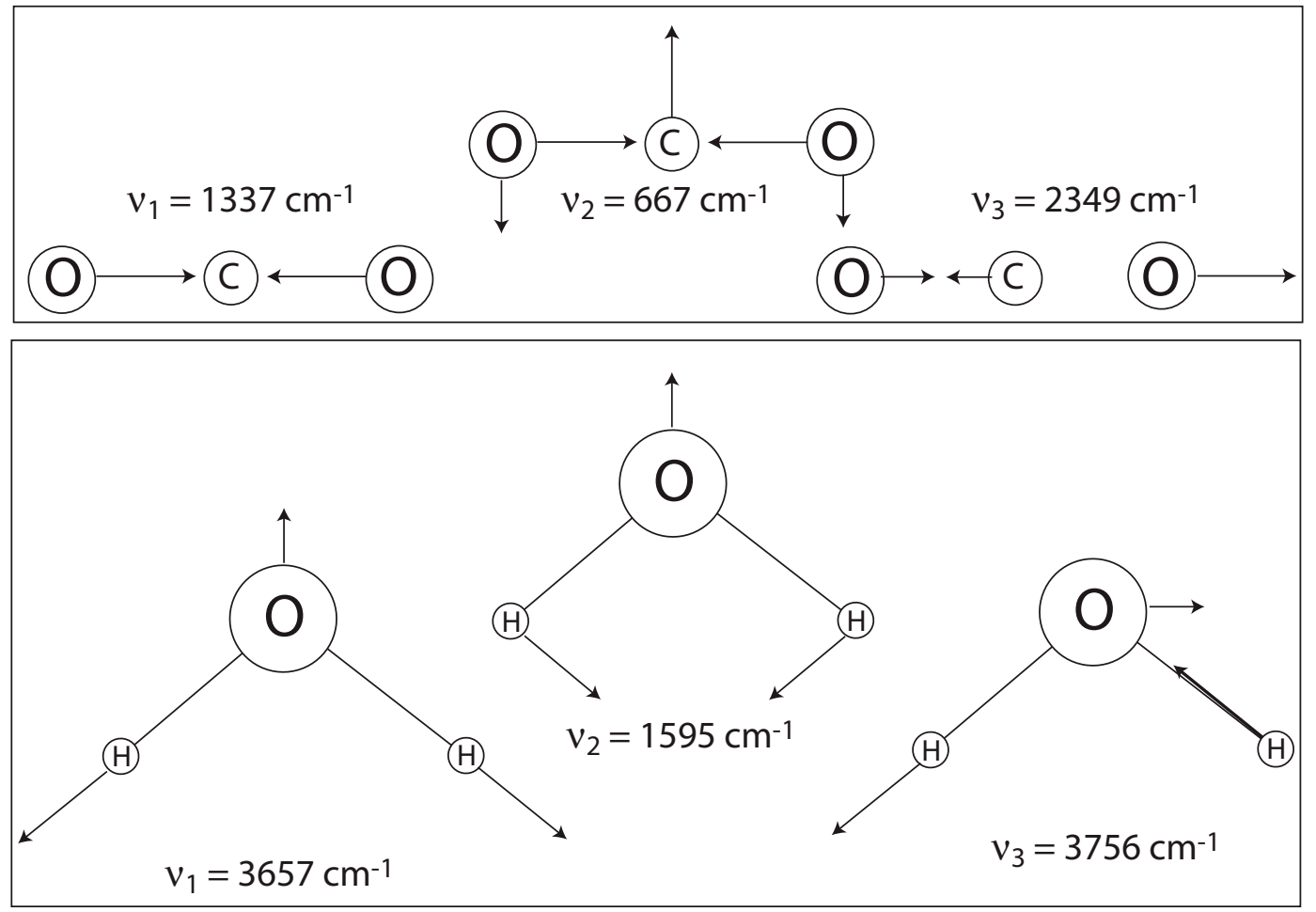

Figure 7. Modes normaux pour la molécule $\mathrm{CO}_{2}$ et pour la molécule d'eau. Les vibrations $v_{1}$ sont des modes symétriques qui sollicitent les liaisons en tension. Les vibrations $v_{2}$ sont des modes symétriques qui plient les angles de liaison. Les vibrations $v_{3}$ sont des modes asymétriques qui tendent les liaisons. Tous ces modes laissent le centre de gravité au repos.

des énergies $E_{n}$ et du nombre de particules $N$ ), ainsi que des champs extérieurs $\vec{E}$ et $\vec{B}$. Dans un ensemble grand-canonique, on introduit le potentiel chimique $\mu$ et la fonction de partition grandcanonique devient

$$
Z=\sum_{n} \exp \left(-\frac{E_{n}-\mu N_{n}}{k_{\mathrm{B}} T}\right),
$$

avec $N_{n}$ le nombre de particules appartenant à l'état $n$. Le potentiel chimique correspond à l'énergie requise pour passer d'un état à $N$ particules à un état à $N+1$ particules.

Pour introduire les probabilités thermiques $p_{n}$ dans le formalisme quantique, il est opportun de définir l'opérateur "densité" :

$$
\hat{\rho}=\frac{e^{-\frac{\mathrm{H}}{k_{\mathrm{B}} T}}}{\operatorname{Tr}\left[e^{-\frac{\mathrm{H}}{k_{\mathrm{B}} T}}\right]},
$$

qui est invariant dans le temps. En combinant la probabilité de la mécanique quantique avec celle de la distribution des états quantiques dans un ensemble thermodynamique (probabilité statistique), on peut exprimer les valeurs moyennes mesurables - c'est-à-dire toutes les fonctions d'état ${ }^{17}$ - sous la

17 Les fonctions d'état définissent l'état d'équilibre d'un système physico-chimique. Elles doivent, de ce fait, ne pas dépendre de l'histoire du système mais uniquement de son état actuel. Le volume $V$, la pression $p$, la température $T$, ainsi que tous les 
forme

$$
\langle A\rangle=\int_{0}^{\infty} p(E) A(E) d E=\sum_{n} p_{n}\left\langle\psi_{n}|\mathbf{A}| \psi_{n}\right\rangle=\operatorname{Tr}[\hat{\rho} \mathbf{A}] .
$$

En particulier, il est possible de déterminer n'importe quel potentiel thermodynamique à partir de la fonction de partition $Z$. Nous donnons ici quelques expressions utiles.

L'énergie interne s'écrit comme

$$
U=-\frac{1}{Z} \frac{\mathrm{d} Z}{\mathrm{~d} \beta}=-\frac{\mathrm{d} \ln Z}{\mathrm{~d} \beta} \quad \text { avec } \beta=\left(k_{\mathrm{B}} T\right)^{-1},
$$

l'énergie libre comme

$$
F=-k_{\mathrm{B}} T \ln Z
$$

l'entropie comme

$$
S=k_{\mathrm{B}} \ln Z+k_{\mathrm{B}} T\left(\frac{\partial \ln Z}{\partial T}\right)_{V},
$$

la pression comme

$$
p=\left(\frac{\partial F}{\partial V}\right)_{T}=k_{\mathrm{B}} T\left(\frac{\partial \ln Z}{\partial T}\right)_{V}
$$

et la chaleur spécifique comme

$$
C_{V}=\left(\frac{\partial U}{\partial T}\right)_{V}=k_{\mathrm{B}} T\left[2\left(\frac{\partial \ln Z}{\partial T}\right)_{V}+T\left(\frac{\partial^{2} \ln Z}{\partial T^{2}}\right)_{V}\right]
$$

La fonction de partition $Z$ condense ainsi toutes les informations requises pour décrire l'état thermodynamique d'un système physico-chimique quelconque. Pour évaluer $Z$ d'un point de vue microscopique on est malheureusement obligé de connaître la totalité du spectre $E_{n}$. Pour calculer des grandeurs physiques selon éq. 2.98 il est même nécessaire de connaître les fonctions d'ondes de tous les états excités. Cela démontre clairement l'intérêt de l'étude de la dynamique, c'est-à-dire des excitations, pour la compréhension de l'état solide.

\subsection{Occupation des niveaux à une particule}

Les probabilités $p_{n}$ exprimées par l'éq. 2.93 se réfèrent aux états stationnaires du système à $N$ particules. Déterminer de tels états et leurs énergies est en géneral un problème intraitable. Dans pratiquement tous les cas, la fonction d'onde du système à $N$ particules est ramenée à une fonction d'onde constituée de particules identiques peuplant des états (niveaux) à une particule. Partout où cela n'est pas possible, on rencontre les grands défis de la théorie de la physique de l'état solide (électrons fortement correlés, supraconductivité etc.). La théorie des bandes électroniques est un exemple typique de théorie qui réduit un problème de plusieurs particules couplées à un problème de particules indépendantes. En dépit du fait que les électrons interagissent fortement entre eux, l'état à $N$ électrons est construit en occupant les niveaux des bandes, en respectant le principe d'exclusion de Pauli, jusqu'au niveau de Fermi (à $T=0$ pour être précis). Les électrons de bande sont des quasi-particules qui incorporent déjà une grande partie des interactions.

potentiels thermodynamiques sont des fonctions d'état. La chaleur transmise à un système ou le travail effectué par un système font partie de son histoire mais ne sont pas des fonctions d'état. Une fonction d'état ne dépend pas du temps. Ceci se reflète dans le fait que l'opérateur densité $\rho$ est invariable dans le temps. 
Il est donc très important de déterminer la probabilité d'occupation d'un niveau à une particule en fonction de la température pour un ensemble thermodynamique. Il faut pour cela distinguer trois grandes classes de particules : les particules identiques discernables, les particules identiques indiscernables de type bosons et les particules identiques indiscernables de type fermions. La différence au niveau de la statistique devient évidente en considérant un système simple de deux particules (notées 1 et 2) qui peuvent occuper deux niveaux à une particule (notés $|0\rangle$ et $|1\rangle$ ). S'il s'agit de deux particules classiques donc discernables- nous avons 4 possibilités de les répartir sur les 2 niveaux $\left(|n\rangle_{i}\right.$ indique que la particule $i$ occupe le niveau $|n\rangle)$ :

$$
|0\rangle_{1}|0\rangle_{2}, \quad|1\rangle_{1}|0\rangle_{2}, \quad|0\rangle_{1}|1\rangle_{2}, \quad|1\rangle_{1}|1\rangle_{2}
$$

Si les particules sont classiques mais indiscernables, il reste trois possbilités

$$
|0\rangle_{1}|0\rangle_{2}, \quad|0\rangle_{1}|1\rangle_{2}, \quad|1\rangle_{1}|1\rangle_{2}
$$

Pour des fermions, la première et la dernière possibilité sont exclues parce qu'un niveau est occupé doublement. De plus, les deux particules étant indiscernables, la fonction est à anti-symétriser par rapport à l'échange des particules. Il reste une seule possibilité

$$
\frac{1}{\sqrt{2}}\left(|1\rangle_{1}|0\rangle_{2}-|0\rangle_{1}|1\rangle_{2}\right)
$$

Dans le cas des bosons, les fonctions sont à symétriser par rapport à l'échange des particules et la double occupation reste permise. On conserve donc trois possbilités.

$$
|0\rangle_{1}|0\rangle_{2}, \quad \frac{1}{\sqrt{2}}\left(|1\rangle_{1}|0\rangle_{2}+|0\rangle_{1}|1\rangle_{2}\right), \quad|1\rangle_{1}|1\rangle_{2} .
$$

Cet exercice peut être rigoureusement étendu à des systèmes de particules en nombre variable. On obtient pour l'occupation d'un niveau à une particule par un fermion, la distribution de Fermi-Dirac

$$
f(E)=\frac{1}{e^{\beta(E-\mu)}+1},
$$

pour les bosons, la fonction de Bose-Einstein

$$
f(E)=\frac{1}{e^{\beta(E-\mu)}-1},
$$

et pour des particules classiques, la distribution de Boltzmann

$$
f(E)=e^{-\beta(E-\mu)},
$$

avec comme avant

$$
\beta=\frac{1}{k_{\mathrm{B}} T}
$$

et $\mu$ le potentiel chimique. Ces fonctions sont représentées sur la figure 8 .

On observe dans les solides différents types d'excitations qui s'étendent sur une large gamme en énergie : des phonons de très basse fréquence -et donc de très faible énergie- aux excitations électroniques de plusieurs électronvolts. Néanmoins, compte tenu de ce qu'on a vu dans la section précédente, elles ne sont pas toutes activées (ou peuplées) à une température donnée. De ce fait elles ne contribuent pas toutes avec le même poids à la valeur moyenne des observables physiques. Pour mieux cerner cet aspect, nous allons regarder de plus près deux systèmes modèles choisis pour leur simplicité et leur pertinence dans l'étude des excitations de réseau : le gaz parfait et l'oscillateur harmonique quantique. 

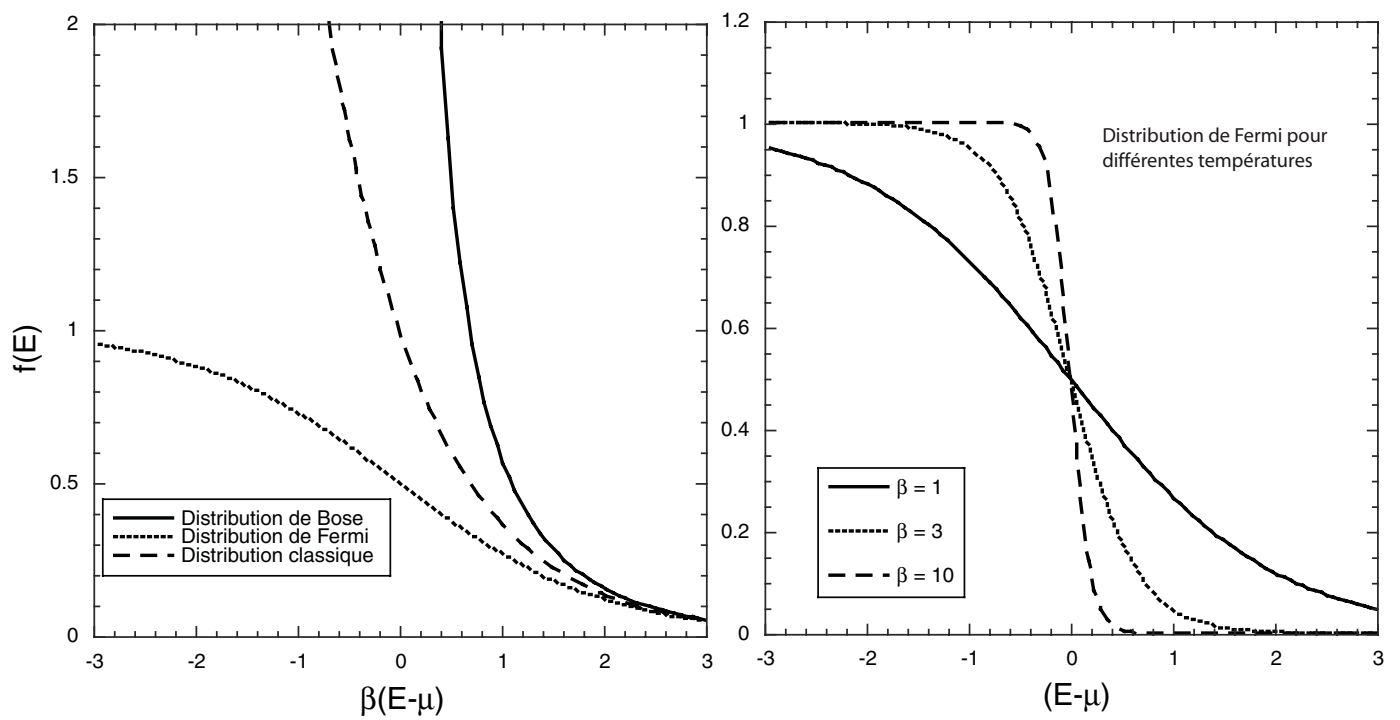

Figure 8. Les différentes fonctions de distribution importantes pour la physique des solides. Figure de gauche: fonction de Fermi, fonction de Bose et fonction de distribution classique. L'énergie est donnée par rapport au potentiel chimique $\mu$ en unité de $\beta^{-1}=k_{\mathrm{B}} T$. Figure de droite: fonction de Fermi pour trois températures différentes. L'unité d'énergie (et donc de $\beta^{-1}=k_{\mathrm{B}} T$ ) est $\mu$. La fonction de Fermi décrit l'occupation d'un niveau d'énergie $E$ pour des fermions. Elle se rapproche de 1 pour des énergies petites comparées au potentiel chimique. L'élargissement autour de $E=\mu$ est de l'ordre de $k_{\mathrm{B}} T$. Pour $T=0$ elle devient une fonction de Heaviside $(f(E)=1$ pour $E \leq \mu$ et $f(E)=1$ pour $E>\mu)$. En accord avec le principe d'exclusion, tous les niveaux en dessous de $\mu$ sont occupés et tous ceux au dessus sont vides. Pour les électrons, le potentiel chimique à $T=0$ est à identifier avec le niveau de Fermi $E_{f}$. La fonction de Bose diverge à $E=\mu$, c'est-à-dire que l'occupation des niveaux de très basse énergie augmente sans limite. Pour éviter cette divergence, il est nécessaire que le plus bas niveau à peupler ait une énergie plus grande que $\mu$. Pour les hautes énergies, les fonctions de distribution se rapprochent de la fonction classique dans les deux cas. Cela s'explique par le fait que pour des nombres d'occupation petits, les occupations multiples sont rares et donc le caractère des particules (exclusion ou non) joue peu sur la statistique.

\subsubsection{Le gaz parfait}

Les molécules qui composent un gaz parfait ne sont pas soumises à des potentiels attractifs. Ceci a pour conséquence que les positions moléculaires sont complètement aléatoires. Les molécules interagissent par collisions élastiques. La mécanique quantique peut introduire des corrélations entre les états occupés en dépit d'une absence d'interaction. Un fermion ne pourra pas occuper un niveau dans lequel se trouve déjà un autre fermion du même type et du même spin. À des températures suffisamment élevées, la probabilité d'une telle double occupation sera petite et nous pourrons négliger les effets quantiques. Dans cette limite, les atomes du gaz sont considerés comme des particules identiques qui peuvent être distinguées. La probabilité $f_{p}$ de trouver un atome de masse $m$ dans un volume $V$ et d'impulsion $\vec{p}$ de coordonnées $\left(p_{x}, p_{y}, p_{z}\right)$, est donc donnée par la distribution de Maxwell-Boltzmann

$$
f_{\vec{p}} d^{3} \vec{p}=f\left(p_{x}, p_{y}, p_{z}\right) d p_{x} d p_{y} d p_{z}=\sqrt{\left(\frac{1}{2 \pi m k_{\mathrm{B}} T}\right)^{3}} \exp \left(-\frac{p_{x}^{2}+p_{y}^{2}+p_{z}^{2}}{2 m k_{\mathrm{B}} T}\right) d^{3} \vec{p}
$$

qui suit directement de la distribution de Boltzmann 2.110 des niveaux. La constante de normalisation précédant l'exponentielle peut être obtenue par intégration (la probabilité de trouver la particule dans 
un état $\vec{p}$ quelconque doit être égale à 1). Cette constante peut être facilement reliée à la fonction de partition pour une particule du gaz parfait

$$
Z=\int_{0}^{\infty} e^{-\beta \hbar^{2} k^{2} / 2 m} g(k) d k=\int_{0}^{\infty} e^{-\beta \hbar^{2} k^{2} / 2 m} \frac{V k^{2}}{2 \pi^{2}} d k=\frac{V}{h^{3}} \sqrt{\left(2 \pi m k_{\mathrm{B}} T\right)^{3}},
$$

où nous avons décrit les particules par des fonctions d'ondes de particules libres comme dans la section 2.1.1, et utilisé la densité d'états exprimée par éq. 2.10. Z pour le gaz parfait s'écrit souvent sous la forme suggestive

$$
Z=\frac{V}{\lambda_{\mathrm{th}}^{3}}
$$

avec la longueur d'onde thermique

$$
\lambda_{\text {th }}=\frac{h}{\sqrt{2 \pi m k_{\mathrm{B}} T}} .
$$

Si nous nous intéressons seulement au module de la vitesse $v=|\vec{v}|$, la distribution devient

$$
f_{v}=4 \pi v^{2} f_{\vec{v}}=4 \pi \sqrt{\left(\frac{m}{2 \pi k_{\mathrm{B}} T}\right)^{3}} v^{2} \exp \left(-\frac{m v^{2}}{2 k_{\mathrm{B}} T}\right)
$$

ce qui nous amène en terme d'énergie cinétique à

$$
f_{E} \mathrm{~d} E=f_{p}\left(\frac{\mathrm{d} p}{\mathrm{~d} E}\right) \mathrm{d} E=\frac{2}{\sqrt{\pi}} \frac{\sqrt{E}}{\left(k_{\mathrm{B}} T\right)^{3 / 2}} \exp \left(-\frac{E}{k_{\mathrm{B}} T}\right) \mathrm{d} E .
$$

$f_{E}$ est représentée dans la figure 9. Le maximum de la distribution du module de la vitesse se situe à

$$
v_{\max }=\sqrt{\frac{2 k_{\mathrm{B}} T}{m}}
$$

La vitesse moyenne est

$$
\bar{v}=\sqrt{\int_{o}^{\infty} v^{2} f_{v} d v}=\sqrt{\frac{3 k_{\mathrm{B}} T}{m}},
$$

ce qui donne comme énergie moyenne

$$
\bar{E}=\frac{m}{2}(\bar{v})^{2}=\frac{3}{2} k_{\mathrm{B}} T .
$$

L'énergie totale d'un gaz parfait de $N$ atomes (ou $n$ moles) est donc donnée par

$$
\bar{E}=\frac{3}{2} N k_{\mathrm{B}} T=n R T,
$$

avec la constante du gaz parfait $R=8.314 \mathrm{~J} / \mathrm{mol} / \mathrm{K}$.

\subsubsection{Les neutrons modérés dans un bain thermique}

Il est ici opportun de faire un petit détour pour regarder de plus près la modération des neutrons. Cette parenthèse nous fera comprendre mieux la raison pour laquelle les neutrons sont des particules idéalement adaptées à l'étude des excitations dans la matière condensée. Les neutrons libres issus, soit de la fission, soit de la spallation de noyaux ont des énergies de plusieurs MeV. Ces neutrons ultrarapides doivent être considérablement "refroidis" (ou "ralentis") pour être utiles dans l'étude de la matière condensée. Ceci est accompli grâce aux modérateurs [7] qui sont constitués d'atomes ou de 


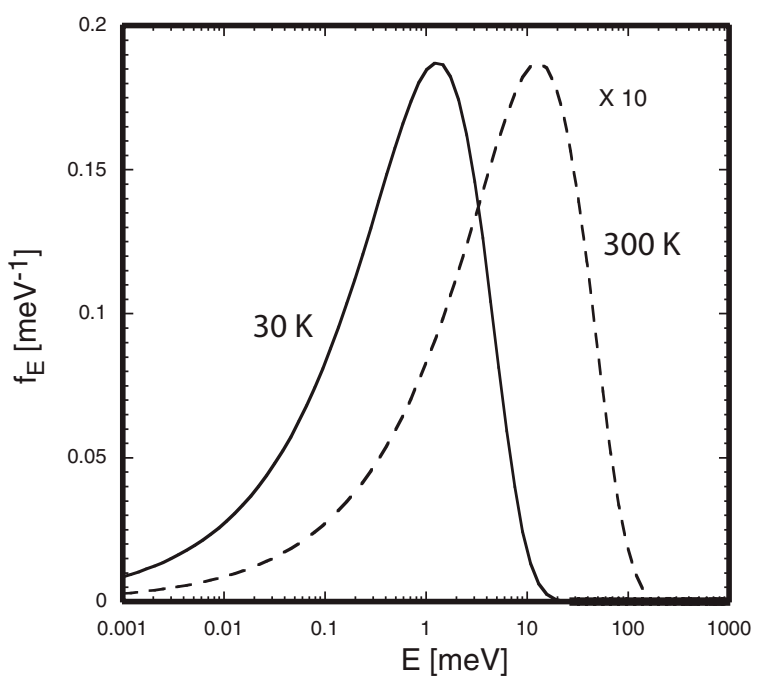

Figure 9. Distribution de Maxwell-Boltzmann pour deux températures, 30 et $300 \mathrm{~K}$, selon l'équation 2.117. La courbe pour $300 \mathrm{~K}$ a été multipliée par un facteur choisi pour permettre une comparaison aisée. Ces distributions correspondent très bien aux spectres de neutrons obtenus avec des modérateurs froids et thermiques.

molécules légers qui diffusent les neutrons sans les absorber. La densité des neutrons étant toujours faible par rapport à la matière condensée ${ }^{18}$, les neutrons peuvent être traités comme un gaz parfait. L'équilibre de ce gaz est ainsi atteint par collisions avec les atomes du modérateur. Par conséquent, le spectre des neutrons modérés est caractérisé par la distribution de Maxwell-Boltzmann à la température du modérateur (éq. 2.117 et figure 9). Le fait que les neutrons aient des énergies comparables aux excitations dans la matière n'est donc pas le fruit du hasard mais le reflet de leur modération dans un bain thermique. L'énergie des neutrons froids (1 à $10 \mathrm{meV})$ va naturellement être plus proche des excitations peuplées à basse température (moins de $100 \mathrm{~K}$ ), les neutrons thermiques (10 à $100 \mathrm{meV}$ ) se prêteront très bien aux études des excitations peuplées à l'ambiante et les neutrons chauds, dont l'énergie est encore plus élevée (100 à $500 \mathrm{meV}$ ), aux excitations peuplées à haute température (plus de $500 \mathrm{~K}$ ). A priori nous aurions dû traiter les neutrons comme des particules quantiques. La distribution de Maxwell-Boltzmann n'est plus appropriée dès que la longueur d'onde thermique $\lambda_{\text {th }}$ est de l'ordre de la séparation des particules. Comme on peut facilement le déduire de l'équation 2.119, la longueur d'onde thermique $\lambda_{\text {th }}$ est très proche de la longueur d'onde moyenne du gaz. Numériquement nous obtenons pour les neutrons

$$
\lambda_{\text {th }}[\AA] \approx \frac{17.4}{\sqrt{T[\mathrm{~K}]}} .
$$

Pour des températures de modération entre 10 et $1000 \mathrm{~K}$ on obtient donc des longueurs d'ondes entre 10 et $1 \AA$. Ces neutrons sont parfaits pour étudier les fluctuations à l'échelle des distances inter-atomiques. Ces longueurs d'onde assez petites impliquent, en outre, que dans tous les cas, la distance entre neutrons, ou mieux la distance parcourue par un neutron entre deux collisions avec des molécules du modérateur, est largement supérieure à $\lambda_{\text {th }}$. Il faudrait des hautes densités de neutrons ultra-froids pour voir naître la nécessité de passer à la distribution quantique de Fermi-Dirac.

\footnotetext{
18 Même pour les sources à très haut flux.
} 
Si on passe du gaz à un neutron spécifique, on obtient la relation entre l'énergie et la longueur d'onde

$$
E[\mathrm{meV}] \equiv 2.0725 k^{2}\left[k \text { in } \AA^{-1}\right] \equiv 81.8204 \lambda^{-2}[\lambda \text { in } \AA]
$$

à partir des relations de de Broglie.

\subsubsection{Thermodynamique de l'oscillateur harmonique}

En décrivant la thermodynamique du gaz parfait, nous n'avons pas vraiment exploité le formalisme de la fonction de partition. Nous allons remédier à cette lacune en traitant le cas de l'oscillateur harmonique. Ainsi que nous l'avons vu dans la section 2.1.2, le spectre de l'oscillateur harmonique quantique est donné par

$$
E_{n}=(n+1 / 2) \hbar \omega .
$$

En exploitant les propriétés des séries géométriques, nous pouvons directement calculer la fonction de partition correspondant à ce spectre

$$
Z=\sum_{n} e^{-\beta E_{n}}=\sum_{n=0}^{\infty} e^{-\beta(n+1 / 2) \hbar \omega}=e^{-(\beta / 2) \hbar \omega} \sum_{n=0}^{\infty} e^{-n \beta \hbar \omega}=\frac{e^{-(\beta / 2) \hbar \omega}}{1-e^{-\beta \hbar \omega}}
$$

Cela nous permet de calculer l'énergie interne en utilisant l'équation 2.99

$$
\begin{aligned}
U=-\frac{d \ln Z}{d \beta} & =\hbar \omega\left[\frac{1}{2}+\frac{1}{e^{\beta \hbar \omega}-1}\right] \\
& =\hbar \omega\left[\frac{1}{2}+f(\hbar \omega)\right],
\end{aligned}
$$

avec $f(\hbar \omega)$ la distribution de Bose-Einstein, qui donne l'occupation moyenne de l'oscillateur. Le terme $(1 / 2) \hbar \omega$ provient des fluctuations de point zéro.

La chaleur spécifique s'obtient en utilisant l'éq. 2.103

$$
C_{V}=\left(\frac{\partial U}{\partial T}\right)_{T}=k_{\mathrm{B}}(\beta \hbar \omega)^{2} \frac{e^{\beta \hbar \omega}}{\left(e^{\beta \hbar \omega}-1\right)^{2}},
$$

l'énergie libre en utilisant l'éq. 2.100

$$
F=-k_{\mathrm{B}} T \ln Z=\frac{\hbar \omega}{2}+k_{\mathrm{B}} T \ln \left(1-e^{-\beta \hbar \omega}\right),
$$

et en utilisant l'éq. 2.101 l'entropie

$$
S=\frac{U-F}{T}=k_{\mathrm{B}}\left(\frac{\beta \hbar \omega}{e^{\beta \hbar \omega}-1}-\ln \left(1-e^{-\beta \hbar \omega}\right)\right) .
$$

Les résultats sont présentés sur la figure 10.

\subsubsection{Equipartition}

Le résultat exprimé par l'éq. 2.121 est intéressant parce qu'il relie de manière directe l'énergie moyenne des atomes à la température. Il peut facilement être transposé à n'importe quel système à $\mathrm{N}$ degrés de liberté, pourvu que ce système puisse être décrit par les lois de la mécanique hamiltonienne. Pour cela on utilisera le théorème d'équipartition, qui stipule que

$$
\left\langle q_{i} \frac{\partial H}{\partial q_{j}}\right\rangle=\left\langle p_{i} \frac{\partial H}{\partial p_{j}}\right\rangle=\delta_{i j} k_{\mathrm{B}} T
$$



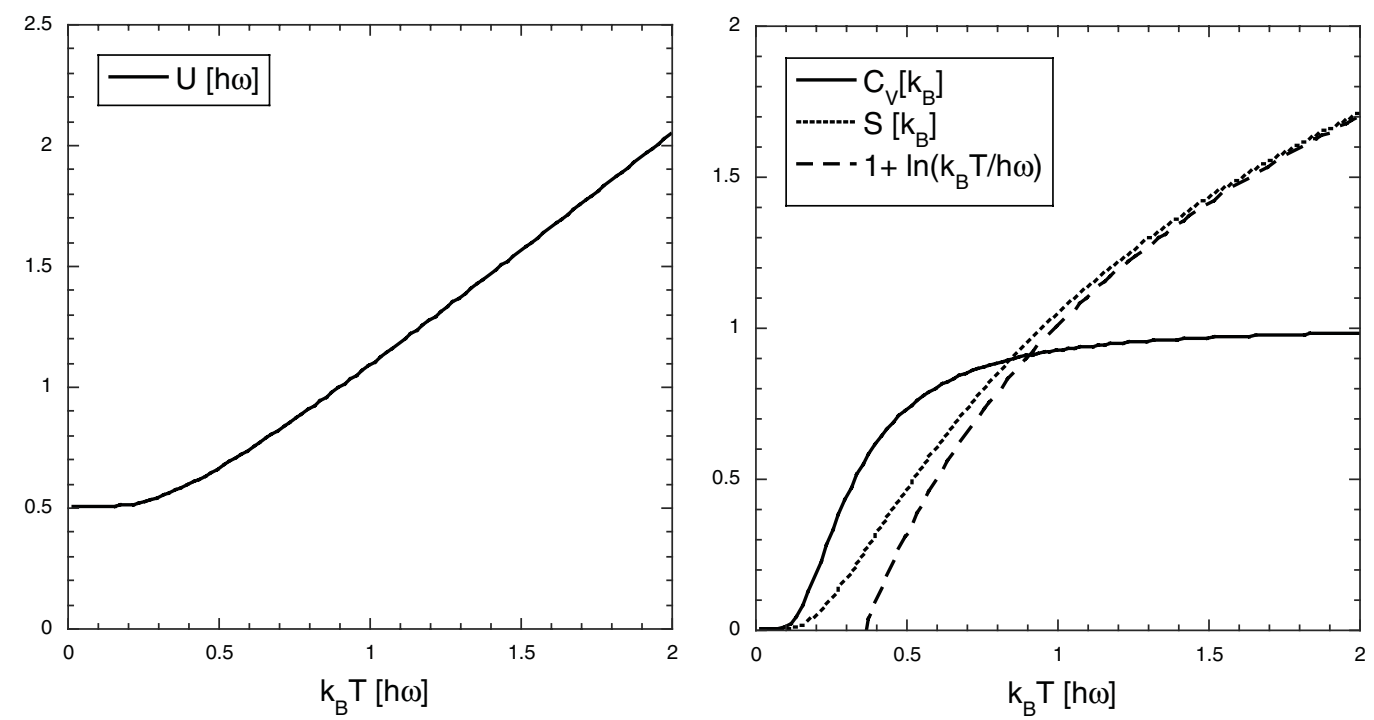

Figure 10. Fonctions thermodynamiques pour un oscillateur harmonique couplé à un bain thermique à la température $T$. L'énergie interne $U$ reste proche de $1 / 2 \hbar \omega$ pour les basses températures et croît de manière linéaire à haute température au fur et à mesure que les niveaux se peuplent. La chaleur spécifique est nulle à basse température et sature à $k_{\mathrm{B}}$ pour les hautes températures. L'entropie est nulle à basse température et augmente comme $1+\ln \left(k_{\mathrm{B}} T / \hbar \omega\right)$. Ceci est compréhensible, puisque l'énergie peut fluctuer d'autant plus que la densité des niveaux est grande $\left(\left(k_{\mathrm{B}} T / \hbar \omega\right)\right.$ pour l'oscillateur à une température donnée). En baissant la fréquence, on augmentera donc l'entropie d'un oscillateur.

avec $\left\{q_{i}\right\}, i=1 \ldots 3 N$ les coordonnées généralisées et $\left\{p_{i}\right\}, i=1 \ldots 3 N$ leurs moments conjugués. Pour des systèmes statiques $(\partial H / \partial t=0)$ l'Hamiltonien $H$ est égal à l'énergie totale $E$, elle-même étant égale à la somme de l'énergie cinétique $T$ et de l'énergie potentielle $V(H=E=T+V)$. Si on se limite à un système de $N$ particules identiques de masse $m$ interagissant par l'intermédiaire d'un potentiel de forme polynomiale

$$
V=\sum_{i} \sum_{s=2}^{\infty} C_{i s} q_{i}^{s}
$$

l'Hamiltonien s'écrit comme

$$
H=\sum_{i} \frac{1}{2 m} p_{i}^{2}+\sum_{i} \sum_{s=2}^{\infty} C_{i s} q_{i}^{s}
$$

Dans ce cas, le théorème d'équipartition appliqué aux moments conjugués permet de calculer la valeur moyenne de l'énergie cinétique ${ }^{19}$ par atome

$$
\left\langle E_{\mathrm{cin}}\right\rangle=\sum_{\alpha=x, y, z} \frac{\left\langle p_{\alpha}^{2}\right\rangle}{2 m}=\frac{1}{2} \sum_{\alpha=x, y, z}\left\langle p_{\alpha} \frac{\partial H}{\partial p_{\alpha}}\right\rangle=\frac{3}{2} k_{\mathrm{B}} T .
$$

\footnotetext{
${ }^{19} \mathrm{Si}$ on prenait des particules relativistes avec $T=c \sqrt{p_{x}^{2}+p_{y}^{2}+p_{z}^{2}}$ on obtiendrait le double $\left\langle E_{\text {cin }}\right\rangle=3 k_{\mathrm{B}} T$.
} 
L'expression qui découle du théorème d'équipartition appliqué aux coordonnées généralisées

$$
\sum_{i}\left\langle q_{i} \frac{\partial H}{\partial q_{i}}\right\rangle=\sum_{i} \sum_{s=2}^{\infty}\left\langle s \cdot C_{i s} q_{i}^{s}\right\rangle=\sum_{i} \sum_{s=2}^{\infty} s \cdot C_{i s}\left\langle q_{i}^{s}\right\rangle=3 N k_{\mathrm{B}} T
$$

n'est en général pas directement liée à l'énergie potentielle moyenne car

$$
\left\langle E_{\mathrm{pot}}\right\rangle=\sum_{i} \sum_{s=2}^{\infty} C_{i s}\left\langle q_{i}^{s}\right\rangle \neq \sum_{i} \sum_{s=2}^{\infty} s \cdot C_{i s}\left\langle q_{i}^{s}\right\rangle,
$$

sauf si le potentiel est de la forme d'une puissance $V=\sum_{i} C_{i r} q_{i}^{r}$. Dans ce cas on peut écrire que l'énergie potentielle moyenne par atome est

$$
\frac{\left\langle E_{\mathrm{pot}}\right\rangle}{N}=\frac{1}{N r} \sum_{i}\left\langle q_{i} \frac{\partial H}{\partial q_{i}}\right\rangle=\frac{1}{N r} \sum_{i} k_{\mathrm{B}} T=\frac{3}{r} k_{\mathrm{B}} T
$$

Le cas le plus important est celui du potentiel harmonique $(r=2)$. Dans ce cas -et seulement dans ce cas- l'énergie totale est partagée à parts égales entre l'énergie cinétique et l'énergie potentielle et chaque degré de liberté du système apporte $\frac{1}{2} k_{\mathrm{B}} T$ à l'énergie totale.

En résumé, et de façon indépendante de la complexité du système, les excitations qui nous intéressent à l'équilibre thermique ont des énergies de l'ordre de quelques $k_{\mathrm{B}} T$. Leur spectre s'échelonne donc entre zéro et quelques centaines de meV pour des températures comprise entre zéro et quelques milliers de Kelvin.

\subsection{Fluctuations, corrélations et réponse linéaire}

Nous avons constaté que toute observable physique en équilibre thermodynamique peut se calculer avec l'aide de l'équation 2.98, pourvu que nous connaissions les états excités du système. Nous avons présenté plusieurs exemples de telles fonctions d'état, qui décrivent le système en équilibre, et qui sont donc par définition invariantes dans le temps. Etant donné que le système est couplé à un réservoir thermique, il est clair que son état va fluctuer dans le temps. Plus le système est petit et plus ces fluctuations vont être prononcées. Il est donc essentiel d'étudier, non seulement la valeur moyenne d'une grandeur physico-chimique (c'est-à-dire à sa fonction d'état associée), mais aussi la distribution de probabilité de cette grandeur. Par exemple, nous pouvons nous demander de combien l'énergie des molécules d'un gaz s'écarte de sa valeur moyenne. Comme toujours en thermodynamique ${ }^{20}$, il est possible et même souhaitable de s'affranchir du temps en étudiant un ensemble représentatif du système.

Il est alors possible de caractériser les fluctuations d'un système $S$ de manière assez générale. Supposons $A$ une fonction d'état quelconque du système $S$ de valeur moyenne à l'équilibre $\langle A\rangle$. Nous cherchons à déterminer la probabilité conditionnelle

$$
p(A, E)
$$

pour que le système d'énergie $E$ se trouve dans un état microscopique compatible avec $A$. Cette probabilité est proportionnelle au nombre de ces états. Nous rappelons que l'entropie - conditionnelle dans ce cas $-S(A, E)$ est le logarithme de ce nombre. Donc

$$
p(A, E) \propto e^{-S(A, E) / k_{\mathrm{B}}} \text {. }
$$

Nous pouvons développer cette expression atour de la valeur moyenne de $A$ comme

$$
S(A, E)=S(\langle A\rangle, E)+\left(\frac{\partial S}{\partial A}\right)_{A=\langle A\rangle} \Delta A+\frac{1}{2}\left(\frac{\partial^{2} S}{\partial A^{2}}\right)_{A=\langle A\rangle}(\Delta A)^{2}+\ldots
$$

\footnotetext{
20 Si le système est ergodique, l'échantillonnage sur un ensemble sera identique à un échantillonnage dans le temps.
} 
avec $\Delta A=A-\langle A\rangle$. A l'équilibre

$$
\left(\frac{\partial S}{\partial A}\right)_{A=\langle A\rangle}=0
$$

et donc

$$
S(A, E)=S(\langle A\rangle, E)+\frac{1}{2}\left(\frac{\partial^{2} S}{\partial A^{2}}\right)_{A=\langle A\rangle}(\Delta A)^{2}+\ldots
$$

En utilisant ce résultat dans éq. 2.138, nous obtenons que

$$
p(A, E) \propto \exp \left(-\frac{(\Delta A)^{2}}{2\left\langle(\Delta A)^{2}\right\rangle}\right),
$$

avec

$$
\left\langle(\Delta A)^{2}\right\rangle^{-1}=\frac{1}{k_{\mathrm{B}}}\left(\frac{\partial^{2} S}{\partial A^{2}}\right)_{A=\langle A\rangle} .
$$

La distribution de $A$ est une gaussienne avec une largeur qui reflète la manière dont l'entropie change avec $A$. Une forte variation de l'entropie avec $A$ indique que le nombre d'états pouvant être parcourus dans l'ensemble diminue fortement dès que $A$ s'éloigne de sa valeur moyenne. Dans ce cas, le système aura tendance à rester proche de sa valeur centrale et la distribution de $A$ sera étroite. ${ }^{21}$

Il est important de souligner que la largeur de la distribution est elle-même une fonction d'état du système. Explicitons la distribution d'énergie interne $U$ d'un système à titre d'exemple. A volume constant, la température est donnée par

$$
\frac{1}{T}=\left(\frac{\partial S}{\partial U}\right)_{V}
$$

et donc

$$
\left(\frac{\partial T^{-1}}{\partial U}\right)_{V}=\left(\frac{\partial^{2} S}{\partial U^{2}}\right)_{V}
$$

Mais

$$
\left(\frac{\partial T^{-1}}{\partial U}\right)_{V}=-\frac{1}{T^{2} C_{V}} .
$$

Combinant l'éq. 2.145 avec l'éq. 2.146 et en utilisant la défnition de l'éq. 2.143, nous aboutissons à

$$
\left\langle(\Delta U)^{2}\right\rangle=k_{\mathrm{B}} T^{2} C_{V}
$$

Nous constatons alors que l'énergie interne du système fluctue. Les fluctuations sont plus prononcées à haute température, de même que pour des systèmes avec une importante chaleur spécifique $C_{V}$. Si nous augmentons la taille du système, la chaleur spécifique va croître de manière linéaire avec le volume. De

\footnotetext{
${ }^{21}$ Une hypothèse fondamentale sous-entendue dans beaucoup de modèles physiques est que les fluctuations peuvent être considérées petites par rapport à la moyenne en dehors du régime critique. C'est par exemple le cas pour un processus qui répond à une distribution de Poisson (voir 3.252). Pour une distribution de ce genre, la largeur est proportionnelle à la racine carrée de la valeur moyenne. La détection des neutrons en est un exemple. Un système de ce type revient vers un état homogène après perturbation. Si on veut rester avec l'exemple des neutrons, le taux de détection devient de plus en plus homogène, c'est-à-dire fiable, avec le temps. En particulier le bruit n'est pas amplifié dans de tels sytèmes mais est au contraire amorti. Il existe des processus qui ne sont pas conformes à ce principe et qui voient l'écart type augmenter avec le temps avec un bruit auto-amplifié. Une petite fluctuation dans un système initialement homogène peut alors produire des grandes inhomogénéités avec le temps. Dans la liste des exemples très intéressants que l'on trouve dans la nature, on trouve le processus d'aggrégation d'organismes microscopiques [8].
} 
ce fait

$$
\frac{\Delta U}{U} \propto \frac{1}{\sqrt{V}}
$$

Les fluctuations relatives vont donc tendre vers zéro pour des systèmes très grands. Ceci est vrai en dehors des transitons de phases pour lesquelles $C_{V} \rightarrow \infty$. Pour ces points critiques, les fluctuations de l'énergie divergent, même pour des systèmes macroscopiques.

\subsubsection{Corrélations}

Nous avons vu que les fluctuations dans un système thermodynamique dépendent de manière cruciale de la taille du système consideré. Nous pouvons franchir un pas de plus et nous demander comment des variables locales sont corrélées entre elles. Statistiquement, deux variables $A$ et $B$ sont corrélées ${ }^{22}$ si

$$
\langle A \cdot B\rangle \neq\langle A\rangle\langle B\rangle \text {. }
$$

Nous pouvons nous en inspirer pour définir

$$
\Gamma_{A, B}(\vec{\rho}, \tau)=\lim _{T \rightarrow \infty} \frac{1}{T} \frac{1}{V} \int_{0}^{T} d t \int_{V} d^{3} \vec{r}\left\langle A(\vec{r}, t) B^{*}(\vec{r}-\vec{\rho}, t-\tau)\right\rangle,
$$

où $A(\vec{r}, t)$ et $B(\vec{r}, t)$ sont des fonctions d'états qu'on pourra évaluer localement. Un exemple typique de telles fonctions est la densité ou le nombre de particules par unité de volume. Mais on pourra aussi choisir une grandeur physique plus complexe comme par exemple l'occupation des états d'un oscillateur harmonique. $\Gamma_{A, B}$ est appelée fonction de corrélation entre $A$ et $B$. Elle permet de comparer les deux fonctions sur une échelle d'espace $\vec{\rho}$ et une échelle de temps $\tau$, par exemple pour en détecter des variations communes. Les domaines d'intégration sur le temps et sur le volume sont importants. Ils assurent que la fonction de corrélation est évaluée en moyennant correctement sur tout le système thermodynamique. Nous rappelons que la valeur moyenne d'une variable $A$ est définie par

$$
\langle A\rangle=\lim _{T \rightarrow \infty} \frac{1}{T} \frac{1}{V} \int_{0}^{T} d t \int d^{3} \vec{r}\langle A(\vec{r}, t)\rangle .
$$

La corrélation dans $\Gamma_{A, B}(\vec{\rho}, \tau)$ est donc evaluée par rapport à un événement déclencheur à l'instant $t$ et à l'endroit $\vec{r}$. En intégrant sur $\vec{r}$ et $t$, nous balayons l'ensemble du système. La distance $\vec{\rho}$ et le temps $\tau$ qui sont les arguments de la fonction de corrélation se réfèrent à la distance et au temps écoulé par rapport à l'événement déclencheur. Dans la majorité des cas, nous nous intéressons aux corrélations d'une variable avec elle-même. La fonction dite d'auto-corrélaton devient ${ }^{23}$ alors

$$
\Gamma_{A, A}(\vec{\rho}, \tau)=\lim _{T \rightarrow \infty} \frac{1}{T} \frac{1}{V} \int_{0}^{T} d t \int d^{3} \vec{r}\left\langle A(\vec{r}, t) A^{*}(\vec{r}-\vec{\rho}, t-\tau)\right\rangle .
$$

Etant donné que nous cherchons à établir des corrélations par rapport à des évènements déclencheurs, à une certaine distance et après un certain laps de temps, il est souvent préférable de faire passer la

\footnotetext{
22 Nous allons reprendre cette discussion dans le chapitre II de cet ouvrage. Les fonctions de corrélations jouent un rôle important dans la théorie de la diffusion des neutrons.

23 On peut se poser la question pourquoi c'est la fonction décalée $A^{*}(\vec{r}-\vec{\rho}, t-\tau)$ qui doit être conjuguée. Dans les faits la conjugaison correspond à une inversion du temps et de l'espace. Comme on peut démontrer par simple changement de variable d'intégration, l'auto-corrélation d'une fonction $f(t)$ peut être calculée de deux façon identiques
}

$$
\begin{aligned}
f \star f & \equiv \int_{-\infty}^{\infty} d t f(t) f^{*}(t-\tau) \\
& =\int_{-\infty}^{\infty} d t f^{*}(t) f(\tau+t) .
\end{aligned}
$$


fonction de corrélation par un filtre qui ne laisse passer que des modulations de longueur d'onde et de fréquence données. Mathématiquement, ce filtrage s'effectue avec l'aide d'une transformation de Fourier dans l'espace et dans le temps. Nous obtenons alors

$$
\Gamma_{A, A}(\vec{Q}, \omega)=\int_{V} d^{3} \vec{r} e^{-i \vec{Q} \cdot \vec{r}} \int_{-\infty}^{\infty} d t e^{-i \omega t} \Gamma_{A, A}(\vec{r}, t) .
$$

Si nous regardons la densité $\rho$ d'un système, la fonction d'auto-corrélation nous renseigne sur la probabilité de trouver une particule à l'endroit $\vec{r}$ au moment $t$, sachant qu'il y avait une particule à l'origine à l'instant $t=0$. Cette probabilité est moyennée sur le temps et sur toutes les particules. Comme nous le verrons plus tard, les neutrons sont une sonde qui permet de mesurer directement cette fonction de corrélation (se référer au chapitre II). Ils sont donc capables de nous renseigner sur la structure et la dynamique de notre système. Le changement de vecteur d'onde et d'énergie définissent les longueurs et les fréquences sondées.

Rien ne nous oblige à nous arrêter à des fonctions de corrélations de deux variables. Pour connaître par exemple la structure d'un liquide, la fonction d'auto-corrélation de la densité est insuffisante. Il faut aussi connaître les probabilités de trouver une particule à l'endroit $\vec{r}$ au moment $t$ étant donné que des molécules se trouvaient aux endroit $\vec{r}_{1}$ et $\vec{r}_{2}$ aux temps $t_{1}$ et $t_{2}$.

\subsubsection{Réponse linéaire}

Dans l'introduction, nous avons montré que le but de toute étude des excitations dans un solide est de comprendre ses fonctionnalités. Jusqu'ici nous avons montré comment les propriétés thermodynamiques peuvent être déduites du spectre des excitations par l'intermédiaire de la fonction de partition. Nous avons inclus dans ces propriétés les fluctuations en fonction du temps et de l'espace. Nous pouvons donc atteindre notre but si nous réussissons à relier la fonctionnalité, c'est-à-dire la réponse d'un système à une perturbation extérieure, aux propriétés thermodynamiques en équilibre, c'est-à-dire aux fluctuations. La base mathématique qui nous permet de franchir ce pas, est la théorie de la réponse linéaire. En partant des principes de la mécanique statistique, elle relie les fonctions de corrélation des observables d'un système à ses propriétés de réponse linéaire [9].

Nous ne pouvons ici que donner un aperçu de cette théorie. Si la perturbation $F$ est petite, le changement induit dans la variable $\hat{A}$ va être en général linéaire par rapport à cette perturbation, c'està-dire qu'il sera proportionnel à l'amplitude et à la durée de la perturbation. Nous pouvons supposer de plus que les perturbations non-simultanées agiront de manière indépendante. La réaction du système à une perturbation ne dépendra de ce fait que du temps écoulé depuis son déclenchement.

Le changement d'une observable physique, exprimée par un opérateur $\hat{A}$, pourra donc formellement être décrit à l'aide d'une fonction de réponse $\phi$ de la manière suivante

$$
\Delta\langle\hat{A}(\vec{r}, t)\rangle=\langle\hat{A}(\vec{r}, t)\rangle-\left\langle\hat{A}_{0}\right\rangle=\int_{-\infty}^{t} d t^{\prime} \int d^{3} \vec{r}^{\prime} \phi\left(\vec{r}, \vec{r}^{\prime} ; t-t^{\prime}\right) F\left(\vec{r}^{\prime}, t^{\prime}\right)
$$

pourvu que les perturbations extérieures $F(\vec{r}, t)$ ne soient pas trop importantes et que le système reste proche de son équilibre thermodynamique. La fonction $\phi\left(\vec{r}, \vec{r}^{\prime} ; t-t^{\prime}\right)$ décrit l'influence sur $\hat{A}$ qu'on ressent à l'endoit $\vec{r}$ au temps $t$, si le champ de $F$ est présent à l'endroit $\vec{r}^{\prime}$ au temps $t^{\prime}$. La réaction est donc en général ni locale dans l'espace ni locale dans le temps, étant donné qu'une perturbation à l'endroit $\vec{r}$ peut toujours entraîner une réponse décalée à un endroit différent. Le principe de causalité requiert néanmoins qu'aucune influence ne puisse être exercée avant $t$, ce qui explique les limites d'intégration dans le temps dans l'équation 2.155.

On peut considérablement simplifier la notation en se limitant à des champs homogènes dans l'espace, ainsi qu'à des observables

$$
\hat{A} \equiv \frac{1}{V} \int d^{3} \vec{r} \hat{a}(\vec{r})
$$


moyennées sur le volume $V$. On obtient une relation purement temporelle de la forme

$$
\Delta\langle\hat{A}(t)\rangle=\int_{-\infty}^{t} \mathrm{~d} t^{\prime} \phi\left(t-t^{\prime}\right) F\left(t^{\prime}\right)
$$

avec

$$
\phi\left(t-t^{\prime}\right)=\frac{1}{V} \int d^{3} \vec{r} \int d^{3} \vec{r}^{\prime} \phi\left(\vec{r}, \vec{r}^{\prime} ; t-t^{\prime}\right) .
$$

On vérifie facilement que pour une perturbation

$$
F\left(t^{\prime}\right)=F_{0} \Delta\left(t^{\prime}\right),
$$

qui est ponctuelle dans le temps, le changement de l'observable est directement donné par la fonction de réponse

$$
\Delta\langle\hat{A}(t)\rangle=F_{0} \phi(t) .
$$

La fonction $\phi(t)$ doit donc mathématiquement être assimilée à la réponse du système à une impulsion.

Pour des systèmes dynamiques linéaires, il est souvent pratique de travailler avec des fonctions périodiques dans le temps, car on peut espérer qu'ainsi, les intégrations -qui ont la forme d'une convolution- se convertissent en simples multiplications. C'est aussi le cas dans le contexte de la réponse linéaire. On doit néanmoins prendre soin de bien définir les transformations, qui nous emmènent de l'espace du temps vers l'espace des fréquences. Ces précautions s'imposent pour prendre en compte à la fois la causalité et la nécessité de maintenir un état d'équilibre thermodynamique.

La définition de la transformée de Fourier de la perturbation

$$
F(\omega)=\int_{-\infty}^{\infty} F(t) e^{i \omega t} d t
$$

et de son inverse

$$
F(t)=\frac{1}{2 \pi} \int_{-\infty}^{\infty} F(\omega) e^{-i \omega t} d \omega
$$

se fait sans difficulté. En ce que concerne la réaction du système, nous sommes confrontés au problème que la perturbation doit se mettre en place de manière adiabatique, c'est-à-dire de manière à laisser le système en permanence dans son état d'équilibre. Cette voie thermodynamique est la seule qui assure une correspondance sans équivoque entre l'état initial et l'état perturbé. Une perturbation périodique ne peut être mise en place de manière adiabatique sauf en intervenant sur son amplitude. Nous définissons par conséquent une transformation de Fourier généralisée

$$
A(\omega)=\langle\hat{A}(\omega)\rangle=\lim _{\epsilon \rightarrow 0^{+}} \int_{-\infty}^{\infty} d t e^{i \omega t} e^{-\epsilon t}(\langle\hat{A}(t)\rangle-\langle\hat{A}(t \rightarrow-\infty)\rangle),
$$

qui prend ce fait en compte.

Il nous reste à établir la fonction qui fait le lien entre $F(\omega)$ et $A(\omega)$. Elle est appelée "susceptibilité généralisée" et se définit à l'aide d'une transformation de Laplace ${ }^{24}$ monolatérale de la fonction de réponse causale $\phi(t)$

$$
\chi[\omega]=\lim _{\epsilon \rightarrow 0^{+}} \chi[i p=\omega+i \epsilon]=\lim _{\epsilon \rightarrow 0^{+}} \int_{0}^{\infty} d t \Phi(t) \exp (p t) .
$$

\footnotetext{
${ }^{24}$ La transformée de Laplace monolatérale d'une fonction $f(t)$ d'une variable réelle positive $t$ est la fonction $F(p)$ de la variable complexe $p$, définie par

$$
F(p)=\int_{0}^{\infty} d t f(t) \exp (-p t)
$$


La notation $[\omega]$ souligne, qu'il s'agit d'une transformée de Laplace monolatérale et non d'une transformée de Fourier. Il peut alors être démontré, à l'aide de l'analyse complexe, que l'expression ${ }^{25}$ 2.157 se convertit en

$$
A(\omega)=\chi[\omega] F(\omega) .
$$

La réponse du système à une perturbation périodique est donc également périodique avec la même fréquence. Nous voudrions souligner que c'est le cas parce que la fonction de réponse est une fonction de $t-t^{\prime}$ et non indépendamment de $t$ et $t^{\prime}$.

La susceptibilité généralisée est une fonction complexe

$$
\chi[\omega]=\chi^{\prime}[\omega]+i \chi^{\prime \prime}[\omega] .
$$

Pour donner un sens au deux composantes, nous regardons la réponse à une perturbation de la forme

$$
F(t)=F_{0} \cos \left(\omega_{0} t\right)
$$

Dans ce cas selon 2.161

$$
F(\omega)=\frac{F_{0}}{2}\left(\delta\left(\omega-\omega_{0}\right)+\delta\left(\omega+\omega_{0}\right)\right) .
$$

En remplaçant ces expressions dans 2.166 nous obtenons la réponse spectrale du système

$$
A(\omega)=\chi[\omega] \frac{F_{0}}{2}\left(\delta\left(\omega-\omega_{0}\right)+\delta\left(\omega+\omega_{0}\right)\right) .
$$

La transformée de Fourier généralisée inverse (voir 2.163) de cette fonction nous ramène dans l'espace temps.

$$
\begin{aligned}
\delta\langle\hat{A}(t)\rangle & =\frac{1}{2 \pi} \int_{-\infty}^{\infty} d \omega e^{-i \omega t} A(\omega) \\
& =\frac{1}{2 \pi} \int_{-\infty}^{\infty} d \omega e^{-i \omega t} \chi[\omega] F(\omega) \\
& =\frac{F_{0}}{4 \pi}\left[\chi\left[\omega_{0}\right] e^{-i \omega_{0} t}+\chi\left[-\omega_{0}\right] e^{i \omega_{0} t}\right] \\
& =\frac{F_{0}}{4 \pi}\left[\cos \omega_{0} t\left\{\chi\left[\omega_{0}\right]+\chi\left[-\omega_{0}\right]\right\}+i \sin \omega_{0} t\left\{\chi\left[\omega_{0}\right]-\chi\left[-\omega_{0}\right]\right\}\right] \\
& =\frac{F_{0}}{2 \pi}\left[\chi^{\prime}\left[\omega_{0}\right] \cos \omega_{0} t+\chi^{\prime \prime}\left[-\omega_{0}\right] \sin \omega_{0} t\right],
\end{aligned}
$$

où nous avons utilisé

$$
\chi^{\prime}[p]=\frac{1}{2}\left(\chi[p]+\chi\left[-p^{*}\right]\right)
$$

et

$$
\chi^{\prime \prime}[p]=\frac{1}{2 i}\left(\chi[p]-\chi\left[-p^{*}\right]\right),
$$

ce qui peut être démontré à partir de la définition 2.165 de la susceptibilité. On vérifie donc que la partie réelle $\chi^{\prime}$ décrit la réponse en phase avec la perturbation (réactive), alors que la partie imaginaire nous renseigne sur la réponse en opposition de phase $^{26}$ ou dissipatrice.

\footnotetext{
25 Le lecteur désireux de s'en convaincre est prié de se référer à la littérature spécialisée, par exemple au livre de Jensen et Mackintosh [15].

${ }^{26}$ Quand la réponse est en phase avec la force motrice, celle-ci ne produit pas de travail. C'est bien connu pour les moteurs électriques. Pour que le moteur fournisse du travail, le rotor aimanté doit être déphasé par rapport au champ magnétique qui l'entraîne.
} 
Il reste à déterminer la susceptibilité $\chi[\omega]$. Pour cela il faut tout d'abord identifier l'opérateur $\hat{B}$ qui couple le champ extérieur $F(t)$ au système. Par exemple, un champ électrique sera couplé à l'opérateur densité de charge et un champ magnétique à l'opérateur densité de moment magnétique. L'Hamiltonien du système perturbé s'écrit alors comme

$$
\hat{H}=\hat{H}_{0}+\hat{H}^{\prime}(t)=\hat{H}_{0}-\hat{B} \cdot F(t) .
$$

Dans le cas d'un système perturbé par un champ magnétique

$$
\hat{H}^{\prime}(t)=\overrightarrow{\hat{M}} \cdot \vec{B}(t),
$$

avec $\overrightarrow{\hat{M}}$ l'opérateur du moment magnétique et $\vec{B}(t)$ le champ appliqué.

On peut démontrer en utilisant les lois de la mécanique statistique [9] que

$$
\phi(t)=\frac{i}{\hbar}\langle[\hat{A}(t), \hat{B}(0)]\rangle \Theta(t)
$$

et ainsi en s'appuyant sur 2.165

$$
\chi[\omega]=\lim _{\epsilon \rightarrow 0^{+}} \chi[i p=\omega+i \epsilon]=\lim _{\epsilon \rightarrow 0^{+}} \int_{0}^{\infty} d t\langle[\hat{A}(t), \hat{B}(0)]\rangle \exp (p t) .
$$

La fonction de Heaviside est définie par $\Theta(t)=1$ pour $t>0$ et 0 ailleurs. Ces équations sont connues sous le nom de lois de Kubo. C'est un résultat d'importance majeure. Ces équations relient les fonctions de corrélations, décrivant les fluctuations thermiques, à la susceptibilité, qui décrit elle-même la réponse du système à une perturbation périodique externe.

Une propriété importante de la susceptibilité, découlant directement de la causalité mentionnée plus haut, est la relation de Kramers-Kronig

$$
\chi^{\prime}[\omega]=P \int_{-\infty}^{\infty} d \omega^{\prime} \frac{1}{\pi} \frac{\chi^{\prime \prime}\left[\omega^{\prime}\right]}{\omega^{\prime}-\omega},
$$

où $P$ désigne la partie principale de Cauchy. La partie réactive de la susceptibilité peut donc être obtenue en intégrant ${ }^{27}$ la partie dissipative sur toutes les fréquences et vice-versa. Connaître une des deux parties est donc a priori suffisant. Le problème d'application de la relation de Kramers-Kronig réside souvent dans le fait que ni l'une ni l'autre partie ne sont connues avec suffisamment de précision sur toute la gamme de $\omega$.

Un cas particulier des lois de Kubo est le théorème de fluctuation-dissipation

$$
\begin{aligned}
S_{x x}(\omega) & =\int_{-\infty}^{\infty} e^{-i \omega t}\langle x(t) x(0)\rangle d t \\
& =2 \hbar(n(\omega)+1) \chi^{\prime \prime}[\omega],
\end{aligned}
$$

qui relie la fonction d'auto-corrélation $S(\omega)$ à la susceptibilité et où le facteur d'occupation thermique est donné par

$$
n(\omega)=\frac{1}{\exp \left(\frac{\hbar \omega}{k_{\mathrm{B}} T}\right)-1} .
$$

Nous allons découvrir que $S(\omega)$ - sous le nom de fonction de diffusion - joue un rôle extrêmement important dans la théorie de la diffusion.

Pour donner un peu de vie à ces formules assez abstraites, et pour en démontrer l'utilité, nous allons présenter plusieurs exemples.

\footnotetext{
27 Il s'agit en général d'une intégrale de Cauchy car souvent l'intégrant diverge pour certaines fréquences.
} 


\subsubsection{Exemple I : oscillateur harmonique}

Nous allons traiter tout d'abord l'oscillateur harmonique amorti et sous l'influence d'une force extérieure. L'équation du mouvement ${ }^{28}$ s'écrit

$$
m\langle\ddot{x}(t)\rangle+m \gamma\langle\dot{x}(t)\rangle+m \omega_{0}^{2}\langle x(t)\rangle=f(t),
$$

où $\gamma$ est la constante d'amortissement et

$$
m \omega_{0}^{2}=K
$$

est la constante de force de rappel. En passant à l'espace de Fourier, l'équation de mouvement se transforme en :

$$
\left(-\omega^{2}+\omega_{0}^{2}+i \gamma \omega\right) x(\omega)=\frac{f(\omega)}{m} .
$$

Cela nous amène à identifier la susceptibilité selon 2.166 avec l'expression

$$
\chi[\omega]=\frac{1}{m} \frac{1}{\omega_{0}^{2}-\omega^{2}-i \gamma \omega} .
$$

La partie imaginaire décrivant la dissipation est donnée par

$$
\chi^{\prime \prime}[\omega]=\frac{1}{m} \frac{\gamma \omega}{\left(\omega_{0}^{2}-\omega^{2}\right)^{2}+(\gamma \omega)^{2}}
$$

et la partie réelle décrivant la partie réactive par

$$
\chi^{\prime}[\omega]=\frac{1}{m} \frac{\left(\omega_{0}^{2}-\omega^{2}\right)}{\left(\omega_{0}^{2}-\omega^{2}\right)^{2}+(\gamma \omega)^{2}} .
$$

$\chi^{\prime \prime}[\omega]$ est une fonction de Lorentz, dont la largeur est déterminée par la constante d'amortissement $\gamma$ (voir figure 11). Il est à souligner que la susceptibilité d'un oscillateur harmonique est strictement indépendante de la température. Ce fait est très utile dans l'interprétation des spectres obtenus avec les neutrons, comme nous allons le voir dans le chapitre III.

Nous pouvons facilement vérifier la relation de Kramers-Kronig pour la partie statique $\chi^{\prime}[0]$

$$
\int_{-\infty}^{\infty} d \omega \frac{\chi^{\prime \prime}[\omega]}{\omega}=\frac{\pi}{m \omega_{0}^{2}}=\frac{1}{K}=\pi \chi^{\prime}[0]
$$

La constante de force de rappel s'exprime en $\mathrm{Nm}^{-1} . \mathrm{K}^{-1}$ définit la variation de la position $\langle x\rangle$ par unité de force appliquée. Elle décrit donc la réponse du système à une perturbation statique.

Connaissant la susceptibilité, le théorème de fluctuation-dissipation nous donne immédiatement accès aux fluctuations de la variable $x$

$$
S_{x x}(\omega)=2 \hbar(1+n(\omega)) \frac{1}{\pi m} \frac{\gamma \omega}{\left(\omega^{2}-\omega_{0}^{2}\right)^{2}+\gamma^{2} \omega^{2}} .
$$

Quand l'amortissement tend vers zéro, les lorentziennes deviennent des fonctions $\delta$ et cette expression peut s'écrire sous la forme

$$
S_{x x}(\omega)=\frac{1}{2 m \omega_{0}}\left[(1+n(\omega)) \delta\left(\omega-\omega_{0}\right)+n(\omega) \delta\left(\omega+\omega_{0}\right)\right],
$$

où nous avons utilisé

$$
1+n(\omega)+n(-\omega)=0 .
$$

\footnotetext{
28 Il est connu que pour un oscillateur harmonique, nous pouvons utiliser les équations classiques pour décrire l'évolution des valeurs moyennes quantiques.
} 

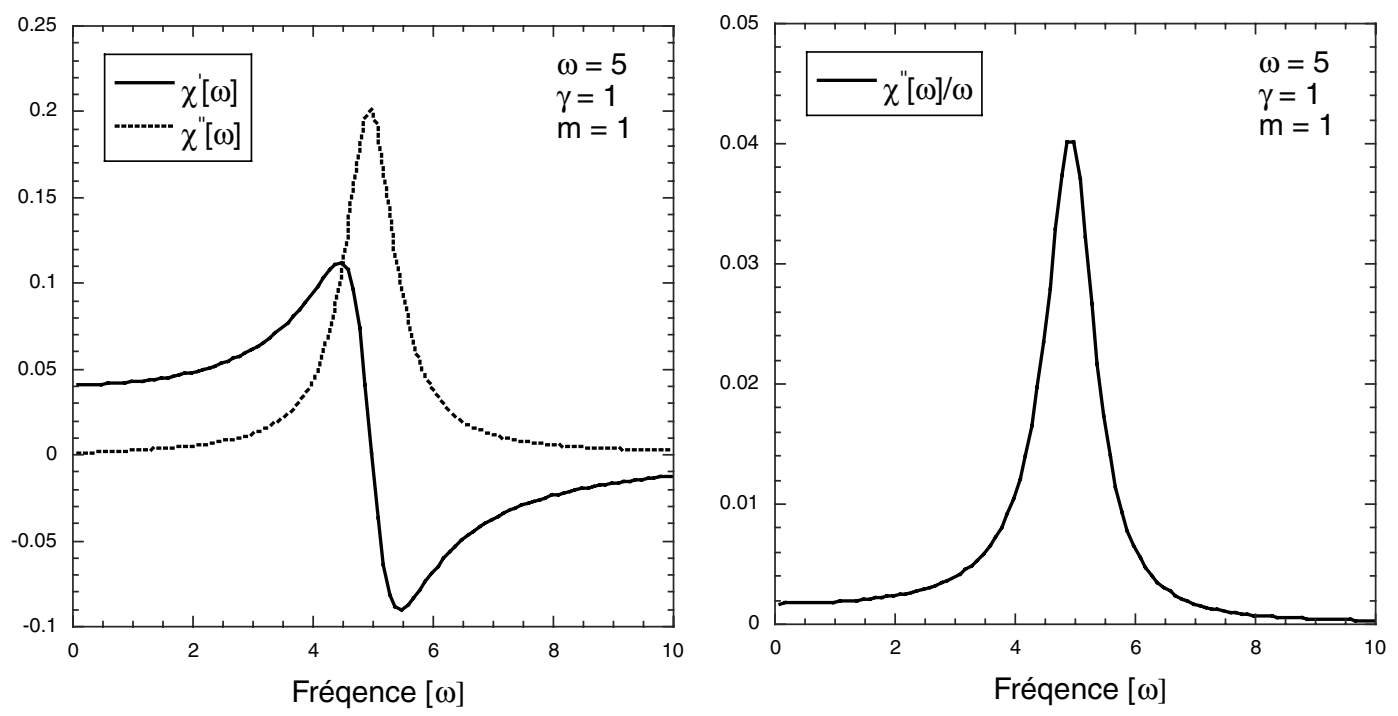

Figure 11. Susceptibilité de l'oscillateur harmonique. A gauche, les parties réelle et imaginaire de $\chi[\omega]$. A droite la fonction $\chi^{\prime \prime} / \omega$. La surface en dessous de cette fonction est égale à $\chi^{\prime}[0]=K^{-1}$.

La partie $\delta\left(\omega-\omega_{0}\right)$ correspond à la création d'une excitation vibrationnelle (augmentation de $1 \mathrm{du}$ niveau $n)$. La partie $\delta\left(\omega+\omega_{0}\right)$ correspond à l'annihilation d'une excitation vibrationnelle (diminution de $1 \mathrm{du}$ niveau $n$ ). L'annihilation n'est pas possible à très basse température car l'oscillateur se trouve dans son état fondamental. Nous allons revenir sur ce point en détail dans le chapitre III lors de la discussion des phonons.

\subsubsection{Exemple II : bruit de Johnson-Nyquist}

Le bruit est un phénomène omniprésent dans la nature. Il s'agit de variations aléatoires (ou autrement dit de fluctuations) d'une grandeur physique. Nous allons ici regarder de plus près le bruit dans une résistance électrique en équilibre thermique. Pour cela nous connectons cette résistance à une charge, c'est-à-dire à une résistance équivalente. Cette adaptation d'impédance assure un maximum de transfert d'énergie (voir figure 12). Nous allons voir que le bruit est un phénomène incontournable régi seulement par la température.

Le long de cette ligne de transmission primitive - considérée comme un système unidimensionneldes ondes électromagnétiques de vecteur d'onde discret $k_{n}$ pourront se propager comme vu à la section 2.1.1. Les vecteurs d'onde s'expriment par

$$
k_{n}=n \frac{2 \pi}{L}, \quad n=0, \pm 1, \pm 2, \pm 3 \ldots,
$$

où $L$ désigne la longueur totale de la ligne. La fréquence de ces modes est reliée à la vitesse de la lumière $c$ par l'intermédiaire de la relation

$$
v_{n}=\frac{1}{2 \pi} c k_{n}=c \frac{n}{L} .
$$

Dans un intervalle de fréquence $\Delta v$ aussi appelé "largeur de bande", nous avons

$$
\Delta v=\frac{c}{L}
$$

le système comporte donc un mode par intervalle $\frac{c}{L}$. 
Chaque mode possède une énergie ${ }^{29} \hbar \omega=h v$ et se trouve peuplé selon le facteur de Bose. Par intervalle $\Delta \nu$, on obtient donc une densité d'énergie - équivalente à une puissance - de

$$
P=\langle E\rangle \Delta v=\frac{h v \Delta v}{\exp \frac{h v}{k_{\mathrm{B}} T}-1},
$$

pour une des deux résistances. En utilisant

$$
P=\left\langle R I^{2}\right\rangle
$$

et

$$
I=\frac{V}{2 R}
$$

on obtient une relation pour les fluctuations de tension créées par la résistance $R$

$$
\left\langle V^{2}\right\rangle=4 R \frac{h v \Delta v}{\exp \frac{h v}{k_{\mathrm{B}} T}-1} .
$$

Pour des fréquences basses, c'est-à-dire pour $h v \ll k_{\mathrm{B}} T$, cette relation s'écrit comme

$$
\left\langle V^{2}\right\rangle=4 R k_{\mathrm{B}} T \Delta \nu
$$

qui est la version classique de l'éq. 2.197. Comme dans le cas de l'oscillateur harmonique, la limite classique est atteinte quand on peut placer un grand nombre de niveaux $h v$ dans un intervalle $k_{\mathrm{B}} T$. La puissance connectée aux fluctuations de tension est

$$
P_{\text {bruit }}=\Delta v k_{\mathrm{B}} T \text {. }
$$

Elle se révèle proportionnelle à la fois à la température et à la bande passante $\Delta v$. Elle est par contre indépendante de la résistance $R$.

La tension mesurable aux bornes de la résistance fluctue donc autour de sa valeur nominale. Ces fluctuations existent indépendamment de toute tension appliquée. Ce bruit électronique thermique est appelée bruit de Johnson et la relation 2.198 est connue sous le nom de relation de Nyquist. Celle-ci connecte les fluctuations de tension à la résistance $R$. Se rappelant que $R^{-1}$ donne la proportionalité entre le courant et la tension appliquée, on se rend compte que $R^{-1}$ décrit la réponse linéaire du système électronique à un champ extérieur. La relation de Nyquist est donc une relation de type Kubo, reliant une réponse à des fluctuations.

Ces fluctuations thermiques sont assez petites comparées aux tensions appliquées ordinairement. Pour une résistance de $1000 \Omega$ à température ambiante $(T=290 \mathrm{~K})$ et pour une bande passante de $\Delta v=1 \mathrm{~Hz}$ on a

$$
\sqrt{\left\langle V^{2}\right\rangle}=4 \times 10^{-9} \mathrm{~V}
$$

En terme de puissance, on obtient

$$
P_{\text {bruit }}[\text { Watt }]=1.38 \cdot 10^{-23} \quad T[\mathrm{~K}] \quad \Delta \nu[\mathrm{Hz}],
$$

ce qui donne à l'ambiante

$$
P_{\text {bruit }}[\text { Watt }]=4 \cdot 10^{-21} \Delta v[\mathrm{~Hz}] .
$$

Pour une télévision classique, utilisant une bande passante de $6 \mathrm{MHz}$, la puissance liée au bruit thermique du récepteur correspond à $24 \cdot 10^{-15}$ Watt, soit un peu plus de $2 \%$ d'un pico-Watt. Ceci est beaucoup plus faible que la puissance du signal reçu par l'antenne. La situation change complètement

\footnotetext{
${ }^{29}$ Nous ferons abstraction de l'indice discret $n$ dans la suite afin d'alléger la notation. Ceci équivaut à considérer de grande longueurs $L$.
} 

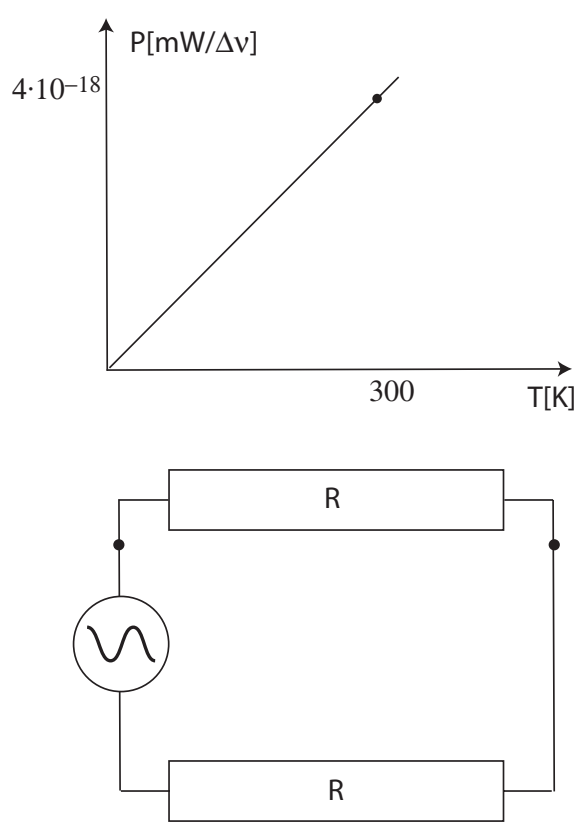
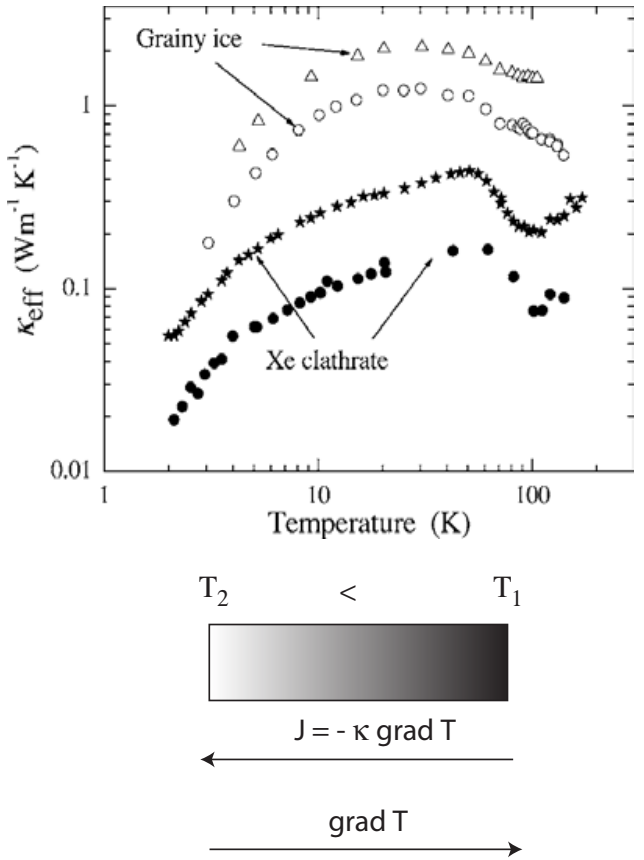

Figure 12. Figure de gauche: circuit équivalent pour décrire le bruit de Johnson. Il consiste en deux résistances identiques $R$ connectées en série donnant une ligne de transmission primitive de longueur $L$. Dans cette ligne, le bruit créé par la résistance $R$ peut être consideré comme une source de tension alternative, mise en série avec la deuxième résistance $R$, qui tient lieu de charge. La puissance dissipée par $R$ est indépendante de $R$. Pour une largeur de bande unité de $1 \mathrm{~Hz}$, elle est de $4 \cdot 10^{-18} \mathrm{mWatt}$ à température ambiante. Figure de droite: le flux de chaleur 77 est opposé au gradient de température $\vec{\nabla} T$. L'exemple montre la constante de conductivité thermique $\kappa$ pour la glace et, pour comparaison, celle d'un clathrate de Xénon [16]. Comme on peut le voir, $\kappa$ dépend de la préparation de l'échantillon. Soustraire ces effets secondaires pour obtenir le $\kappa$ intrinsèque du matériau est un des défis à relever par l'expérimentateur.

pour le cas du GPS, dont le signal détecté a une puissance de quelques $10^{-16}$ Watts $^{30}$ pour une bande passante de $2 \mathrm{MHz}$. Il peut être détecté seulement grâce à un traitement sophistiqué du signal. Le même phénomène de bruit thermique discuté ici pour une résistance $R$ est observé aux bornes d'une capacité $C$. Ce bruit capacitif constitue une limitation des capteurs photographiques.

\subsubsection{Exemple III : la conductivité thermique}

Dans l'exemple précédent, nous avons mené la discussion du point de vue des fluctuations, même si ces fluctuations ont finalement été reliées à la résistivité électrique du matériau qui constitue une propriété

\footnotetext{
${ }^{30}$ Un satellite GPS envoi un signal de 50 Watt. Il y a 24 satellites qui couvrent l'ensemble de la terre. Quatre d'entre eux peuvent être reçus simultanément. Les 50 Watts sont donc répartis sur plus ou moins un sixième de la surface terrestre. Le récepteur GPS doit recevoir son signal sur une surface de quelques $\mathrm{cm}^{2}$ ce qui correspond à quelques $10^{-16}$ Watt. La situation est très similaire à une expérience de neutrons. Une source puissante de neutrons comme celle de l'ILL fournit à peu près un Watt de neutrons thermiques. Parmi eux, $10^{6}$ arrivent par seconde sur un échantillon de un $\mathrm{cm}^{2}$ sur un instrument comme IN6. L'énergie d'un neutron de $5 \mathrm{meV}$ étant $8 \cdot 10^{-22}$ Joule, ce flux correspond à plus ou moins $10^{-15}$ Watt. Parmi ces neutrons, $10 \%$ sont diffusés dans un détecteur de plusieurs $\mathrm{m}^{2}$. La sensibilité d'une expérience de neutrons, en terme d'énergie cinétique détectée par unité de temps, est donc de quelques ordres de grandeur plus élevée que celle d'un récepteur GPS. Ceci est possible grâce au fait que l'énergie dégagée lors de l'absorption du neutron, et qui ensuite est disponible pour effectuer la détection, est beaucoup plus grande que l'énergie cinétique du neutron. Cela a néanmoins l'inconvénient d'interdire toute détection sensible à l'énergie cinétique (energy sensitive detection), qui rendrait l'instrumentation neutronique beaucoup plus efficace.
} 
de la réponse de ce système. Dans le cas de la conductivité thermique, nous allons prendre l'approche inverse : en partant de la grandeur de transport, nous tâcherons d'établir la liaison avec les fluctuations à l'équilibre.

La conduction thermique est un phénomène de transport d'énergie sans transport global de masse, provoqué par un gradient de température au sein d'un même milieu, ou entre deux milieux en contact. Elle est d'importance considérable dans beaucoup de domaines scientifiques, de la formation des clathrates au bilan thermique des composants électriques en passant par les matériaux thermoélectriques. Le transport thermique s'effectue par l'intermédiaire d'échanges d'énergie cinétique entre les particules composant le matériau. Dans un métal, en plus des ions (par l'intermédiaire de leurs vibrations (phonons)), les électrons de conduction contribuent à la conductivité thermique. Dans ce chapitre, nous négligerons cet aspect électronique. Même en négligeant les électrons, le mécanisme exact du transport de chaleur par les excitations du réseau est toujours mal compris dans le détail.

Soit $\vec{J}$ le vecteur du flux de chaleur. $|\vec{J}|$ indique la quantité de chaleur qui traverse une unité de surface perpendiculaire à $\vec{J}$ pendant l'unité de temps. $\vec{J}$ s'exprime donc en Watt $/ \mathrm{m}^{2}$. La conductivité de chaleur est caracterisée, en régime linéaire (voir figure 12), par la constante de conductivité thermique $\kappa$ selon la relation

$$
\vec{J}=-\kappa \vec{\nabla} T
$$

Le calcul de $\kappa$ est assez direct dans un gaz. Dans ce cas, le transport de chaleur se fait par le mouvement des molécules qui échangent de l'énergie cinétique lors des collisions statistiques. ${ }^{31}$ On obtient

$$
\kappa_{\mathrm{gaz}}=\frac{1}{3} C_{V} \lambda\langle v\rangle
$$

où $C_{V}$ est la chaleur spécifique à volume constant, $\lambda$ le libre parcours moyen entre collisions et $\langle v\rangle$ la vitesse moyenne.

Ce modèle peut se généraliser à un solide à l'aide de la théorie cinétique des phénomènes de transport sous l'approximation du temps de relaxation $[11,12]$. En se limitant à la contribution du réseau, cette théorie donne la formule de Debye :

$$
\kappa(T)=\frac{1}{3} C(T) v_{\mathrm{ph}} l_{\mathrm{ph}}=\frac{1}{3} C(T) v_{\mathrm{ph}}^{2} \tau_{\mathrm{ph}},
$$

où $v_{\mathrm{ph}}(T), l_{\mathrm{ph}}(T)$ et $\tau_{\mathrm{ph}}(T)$ sont la vitesse moyenne, le libre parcours moyen et le temps de diffusion moyen des phonons qui transportent la chaleur.

La théorie cinétique est incapable de rendre compte des détails de la conduction thermique dans des systèmes complexes. Elle est, par exemple, incapable d'expliquer pourquoi il y a une telle disparité entre la conductivité de la glace ordinaire et des clathrates [14] (voir figure 12) en fonction des différences entre les phonons. Pour simplifier, il est souvent souhaitable de travailler à l'équilibre thermodynamique. Cela vaut en particulier pour la modélisation à l'aide de la dynamique moléculaire. Les lois de Kubo appliquées à la conductivité thermique donnent [13]

$$
\kappa=\frac{V}{3 k_{\mathrm{B}} T^{2}} \int_{0}^{\infty} \sum_{i=x, y, z}\left\langle J_{i}(t) J_{i}(0)\right\rangle d t
$$

pour des systèmes cubiques ou isotropes. La conductivité thermique peut donc se calculer à partir de la fonction d'auto-corrélation du flux thermique $\langle\vec{J}(t) \vec{J}(0)\rangle$ à l'équilibre, c'est-à-dire sans être obligé de décrire un système soumis à un gradient de température. L'avantage de cette approche devient évident

\footnotetext{
${ }^{31}$ Le transport de chaleur ressemble donc beaucoup à la diffusion et on obtient des résultats très similaires à ceux obtenus pour la viscosité d'un fluide ou la diffusion de particules.
} 
dans le cas des simulations par dynamique moléculaire. En utilisant la formule

$$
\vec{J}=\frac{1}{V}\left[\sum_{i} \vec{v}_{i}\left(E_{i}-\left\langle E_{i}\right\rangle\right)+\sum_{i j} \vec{r}_{i j}\left(\vec{v}_{j} \cdot \frac{\partial V_{i}}{\partial \vec{r}_{j}}\right)\right]
$$

où $i$ et $j$ se réfèrent aux atomes de la boîte de simulation, cette fonction d'auto-corrélation est pleinement accessible à la simulation. ${ }^{32}$

Le premier terme dans cette expression rappelle beaucoup la théorie cinétique. Il décrit le transport de l'énergie par le mouvement balistique des particules entre les différents pas de simulation, d'où son caractère "convectif". Le deuxième terme est appelé "conductif". Ce formalisme permet d'identifier la contribution des différents canaux de conduction au transport de la chaleur, au moins pour des systèmes modèles. Nous en donnerons un exemple dans la section 4.3 après avoir introduit la notion d'excitations du réseau.

\section{DYNAMIQUE DES RÉSEAUX}

Nous allons maintenant déterminer le spectre des excitations dans un réseau ionique. Le point de départ est nécessairement la structure. Une structure est non-triviale s’il existe des corrélations entre les positions des atomes ou des molécules. Ainsi, les fluides ont une structure due aux interactions attractives ou répulsives - qui font que des couches de corrélation se développent autour d'une molécule. Ces corrélations structurales diminuent avec la distance et ont une durée de vie finie. A l'échelle macroscopique, la structure d'un fluide est isotrope et homogène. Mis à part l'hélium ${ }^{33}$, tout fluide devient solide à des températures suffisamment basses. Il peut soit cristalliser via une transition de phase du premier ordre, soit devenir un verre. Dans ce dernier cas, le point en temps et température au delà duquel on considère que le système est à l'état solide est arbitraire et dépend de la durée de l'observation expérimentale et de l'historique du refroidissement. Nous ne souhaitons pas explorer plus avant ce sujet complexe et d'un intérêt scientifique certain mais nous supposerons, lors des considérations théoriques ultérieures, que nous avons affaire à des matériaux complètement relaxés où le processus de formation est terminé, et qui n'évoluent plus. Ceci signifie que les positions atomiques - moyennées dans le temps sur les périodes des vibrations- ne changent pas durant toute la durée de l'expérience. Cela exclut de facto la diffusion des atomes à l'échelle des périodes des vibrations atomiques.

Nous voulons souligner que l'observation de corrélations statiques, c'est-à-dire stables dans le temps, ne signifie nullement l'absence de dynamique. Même à très basse température, les fluctuations quantiques - déjà rencontrées dans le traitement de l'oscillateur harmonique (section 2.1.2)- entraînent une distribution des positions atomiques autour des valeurs moyennes. Ceci devient très important dans le cas des "solides quantiques", comme les cristaux de $\mathrm{H}_{2}$ et $\mathrm{D}_{2}$ : dans l'état fondamental du cristal, les molécules $\mathrm{D}_{2}$ ont un moment rotationel $J=0$. Leur fonction d'onde est donc sphérique, c'est-à-dire que la direction de l'axe moléculaire n'est pas définie dans l'espace [18].

\footnotetext{
32 On doit préciser que la formule donnée n'est correcte que dans le cas où l'Hamiltonien peut s'écrire, avec une bonne approximation, comme

$$
\hat{H}=\sum_{i}\left(\frac{1}{2} m_{i} v_{i}^{2}+V_{i}\right)=\sum_{i} \hat{h}_{i}
$$

c'est-à-dire comme une somme d'Hamiltoniens de particules indépendantes $i$ exposées à des potentiels $V_{i}$. Ce détail est sans importance pour la discussion générale menée ici.

33 L'hélium devient solide à des pressions supérieures à 26 bars [17].
} 


\subsection{Approximation adiabatique}

Etant donné que les positions moyennes des atomes sont stables, les noyaux se trouvent nécessairement dans un minimum de potentiel. Les positions des noyaux vont fluctuer autour de ces positions d'équilibre, même à zéro Kelvin par suite des oscillations quantiques. On se rappelle la fonction d'onde de l'oscillateur harmonique dans l'état fondamental (section 2.1.2). En principe, le mouvement des noyaux ioniques (noyaux plus électrons de cœur) et celui des électrons de valence sont couplés. Il ne faut pas oublier que l'interaction entre atomes se fait par l'intermédiaire des électrons. C'est le recouvrement des fonctions d'onde des électrons qui est responsable des liaisons chimiques. Par conséquent, toute étude de la dynamique des ions est d'abord une étude du système électronique ${ }^{34}$. Comme nous allons le voir plus loin, le couplage dynamique entre ions et électrons est à la base de la supraconductivité ou des transitions métal-isolant.

Mathématiquement, le couplage s'exprime par le fait que l'opérateur de Hamilton $H$ contient à la fois les degrés de liberté ioniques $\left\{\vec{R}_{I}\right\}$ et électroniques $\left\{\vec{r}_{i}\right\}$ comme variables dynamiques, menant à une équation de Schrödinger de la forme

$$
i \hbar \frac{\partial}{\partial t} \Psi\left(\left\{\vec{R}_{I}\right\},\left\{\vec{r}_{i}\right\} ; t\right)=H \Psi\left(\left\{\vec{R}_{I}\right\},\left\{\vec{r}_{i}\right\} ; t\right)
$$

avec l'opérateur de Hamilton ${ }^{35}$

$$
\begin{aligned}
H & =\sum_{i} \frac{\hbar^{2}}{2 m_{i}} \nabla_{i}^{2}+\sum_{I} \frac{\hbar^{2}}{2 M_{I}} \nabla_{I}^{2}+\sum_{i \leq j} \frac{e^{2}}{\left|\vec{r}_{i}-\vec{r}_{j}\right|}+\sum_{I \leq J} \frac{Z_{I} Z_{J} e^{2}}{\left|\vec{R}_{I}-\vec{R}_{J}\right|}+\sum_{i, I} \frac{Z_{I} e^{2}}{\left|\vec{r}_{i}-\vec{R}_{I}\right|} \\
& =T_{i}+T_{I}+V_{i, j}+V_{I, J}+V_{i, I} .
\end{aligned}
$$

$T_{i}$ représente l'énergie cinétique des électrons, $T_{I}$ l'énergie cinétique des ions, $V_{i, j}$ l'interaction électron-électron, $V_{I, J}$ l'interaction ions-ions, et $V_{i, I}$ l'interaction électron-ion. Les solutions stationnaires, c'est-à-dire l'état fondamental et les états excités, sont données par les fonctions propres de $H$ vérifiant l'équation aux valeurs propres

$$
H \Psi_{n}\left(\left\{\vec{R}_{I}\right\},\left\{\vec{r}_{i}\right\} ; t\right)=E_{n} \Psi_{n}\left(\left\{\vec{R}_{I}\right\},\left\{\vec{r}_{i}\right\} ; t\right),
$$

les $E_{n}$ étant les énergies d'excitation du système couplé. Même si les ions étaient considérés comme stationnaires, résoudre seulement le problème électronique constituerait déjà une tâche formidable. Une résolution directe de l'équation 3.4 est sans espoir, à part pour des clusters de quelques atomes. Heureusement, une solution exacte n'est presque jamais nécessaire pour comprendre la physique du système. Cela vaut en particulier pour l'étude du mouvement des ions. Le rapport de masse implique que la dynamique des électrons est en général beaucoup plus rapide que celle des ions. Par conséquent, les électrons ont la faculté de s'adapter d'une manière instantanée aux changements du système ionique (voir figure 13 pour une illustration simple). Dans ce cas, on pourra factoriser la fonction d'onde de l'ensemble, et l'exprimer comme un produit

$$
\Psi\left(\left\{\vec{R}_{I}\right\},\left\{\vec{r}_{i}\right\} ; t\right)=\psi\left(\left\{\vec{r}_{i}\right\}, t ;\left\{\vec{R}_{I}\right\}\right) \Phi\left(\left\{\vec{R}_{I}\right\} ; t\right)
$$

avec une équation pour la partie électronique,

$$
\left(T_{i}+V_{i, j}+V_{i, I}\right) \psi\left(\left\{\vec{r}_{i}\right\}, t ;\left\{\vec{R}_{I}\right\}\right)=E_{\mathrm{el}}\left(\left\{\vec{R}_{I}\right\}\right) \psi\left(\left\{\vec{r}_{i}\right\}, t ;\left\{\vec{R}_{I}\right\}\right),
$$

\footnotetext{
34 On peut trouver une introduction aux vibrations dans les cristaux dans les livres de Kittel [2] ou Ashcroft et Mermin [1]. Une discussion complète est donnée dans la référence [20].

35 Nous utiliserons dans ce chapitre des indices majuscules pour les ions et minuscules pour les électrons. $Z_{I}$ et $M_{I}$ désignent donc respectivement les charges et masses ioniques. Dans tous les cas où il n'y a pas d'ambiguïté, nous abandonnerons cette notation peu pratique.
} 

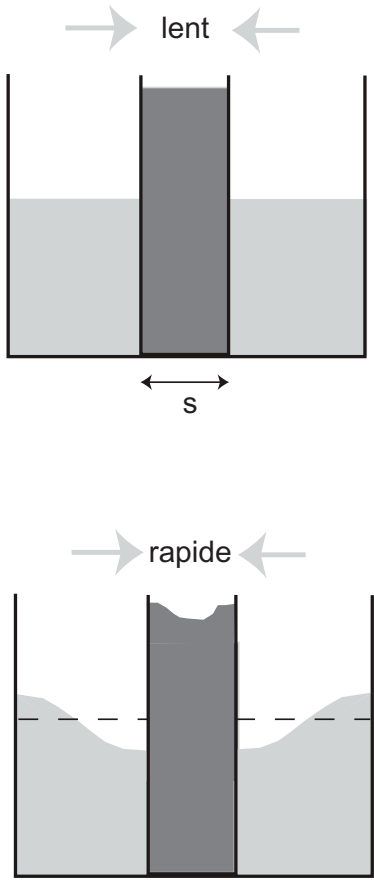

Adiabatique

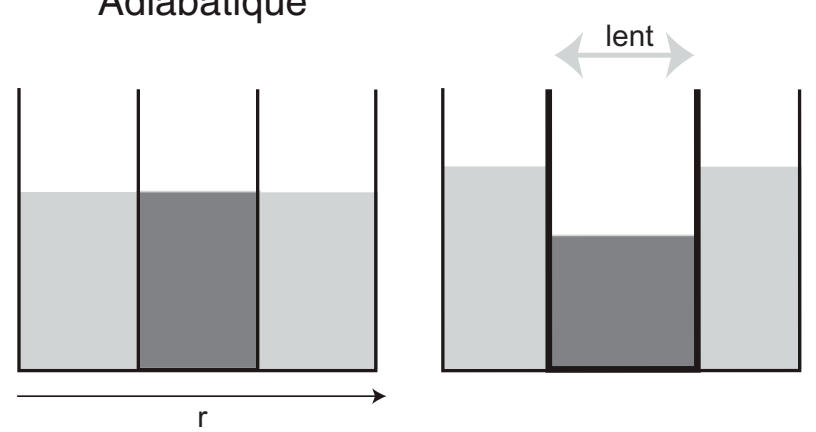

Non-Adiabatique
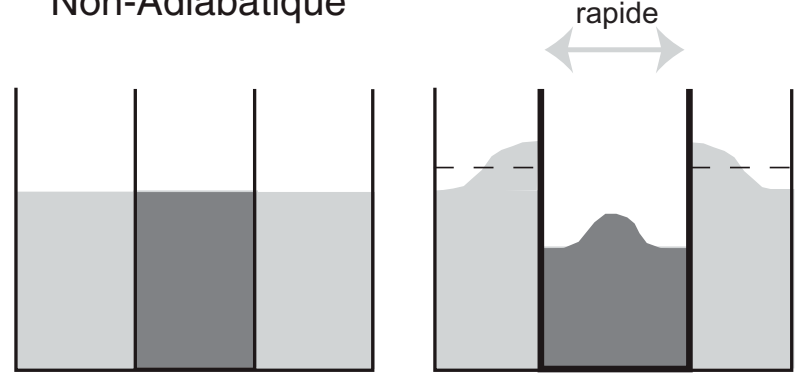

Figure 13. Représentation schématique de l'approximation adiabatique. Dans l'exemple choisi, deux membranes rigides mais mobiles séparent les parties intérieures et extérieures d'un réservoir. A l'équilibre, les parois s'adaptent aux forces exercées et le niveau du liquide est partout identique. Quand les parois sont déplacées, les niveaux montent dans les parties comprimées du réservoir et baissent ailleurs. Si la viscosité du liquide est faible, les molécules vont très vite trouver l'état d'équilibre global. La surface du liquide va donc spontanément suivre le déplacement des parois pourvu que celui-ci ne soit pas trop rapide. C'est la situation adiabatique. Le niveau du liquide correspond à une énergie potentielle $E(s)$ qui ne dépendra que de la séparation des parois $s$. Dès qu'on change les positions des parois rapidement, la situation se complique : des oscillations propres du liquide apparaissent. Pour décrire mathématiquement le système dans ce cas, il faudrait connaître le niveau $E(r, t)$ du liquide pour tous les points $r$ à tous les temps $t . E(r, t)$ va nécessairement être une fonction, non seulement de la séparation, mais aussi de la fréquence d'oscillation $\omega$ des parois: $E(r, t)=E(r, t, s ; \omega)$. La fréquence va être principalement déterminée par la masse des parois et la densité du liquide. Si cette oscillation est en résonance avec l'excitation (les parois qui bougent), on court droit à la catastrophe. Si on laisse le système perturbé extérieurement se développer en toute autonomie, il naîtra une oscillation propre. Celle-ci sera amortie du fait de la viscosité du liquide. En fonction de la fréquence et de la viscosité, cette oscillation préservera des surfaces planes. Celles-ci correspondent à la situation adiabatique où le système pourra êre décrit comme une particule dans un potentiel $E(s)$.

qui ne contient plus les coordonnées des ions comme variables dynamiques, mais seulement comme paramètres, et une équation pour les ions

$$
\left(T_{I}+E_{\mathrm{el}}\left(\left\{\vec{R}_{I}\right\}\right)+V_{I, J}\right) \Phi\left(\left\{\vec{R}_{I}\right\}, t\right)=E \Phi\left(\left\{\vec{R}_{I}\right\}, t\right),
$$

dans laquelle l'énergie électronique $E_{\mathrm{el}}\left(\left\{\vec{R}_{I}\right\}\right)$ est prise en compte comme potentiel externe. En général, cette approximation dite adiabatique [1] marche bien pour la plupart des matériaux. Néanmoins, il serait prudent à ce propos de ne pas considérer cette approximation comme allant de soi. Dans un système quantique, rien n'empêche de coupler une excitation électronique à une excitation ionique, c'est-à-dire d'imaginer une résonance entre les deux, sauf dans les cas où les fréquences sont très éloignées les unes des autres. Pour les excitations, cela implique une séparation claire des échelles 
d'énergie. C'est la situation qu'on rencontre dans les isolants où la séparation entre la bande de valence et la bande de conduction est de l'ordre de plusieurs $\mathrm{eV}$, tandis que les énergies des excitations ioniques (vibrations, phonons) ne dépassent guère quelques centaines de meV. Dans les métaux, l'argument est moins évident parce que des excitations proches de la surface de Fermi peuvent avoir des énergies arbitrairement petites. Rien ne s'oppose a priori à ce que ces excitations se couplent aux excitations ioniques. L'approximation adiabatique est donc douteuse. En dépit de cette faiblesse d'argumentation, l'approximation adiabatique marche très bien dans la plupart des métaux ${ }^{36}$, mais des exceptions existent et la plus connue est certainement le phénomène de la supraconductivité. La raison pour laquelle l'approximation adiabatique marche habituellement doit être cherchée dans les effets négligés ou sommairement traités par le concept de bandes électroniques ${ }^{37}$. La diffusion des électrons par l'intermédiaire de collisions, conjuguée à l'écrantage électronique, jouent un rôle important dans l'adaptation locale et rapidement amortie des électrons aux changements de la configuration ionique.

\subsection{Approximation harmonique}

Ayant séparé les électrons des ions, nous allons maintenant déterminer le caractère des excitations du réseau ionique d'un solide, dans lequel les atomes se trouvent ancrés dans les puits d'un potentiel donné par l'expression (éq. 3.7)

$$
U\left(\vec{R}_{1}, \ldots \vec{R}_{N}\right)=E_{\mathrm{el}}\left(\left\{\vec{R}_{I}\right\}\right)+V_{I, J} .
$$

Les vecteurs positions $\vec{R}_{n}$ sont des quantités qui dépendent du temps. Dans un solide, les $N$ positions d'équilibre des ions sont indépendantes du temps. Nous les noterons $\vec{R}_{n}^{0}, n=1, \ldots, N$ dans la suite et noterons $\vec{u}(n \mid t)$ le déplacement de l'ion $n$ par rapport à sa position d'équilibre $R_{n}^{0}$ au temps $t$. Il est possible de développer le potentiel $U\left(\vec{R}_{1}, \ldots \vec{R}_{N}\right)$ en fonction des déplacements ioniques $\vec{u}(n \mid t)$ tel que

$$
\vec{u}(n \mid t)=\vec{R}_{n}-\vec{R}_{n}^{0}, \quad n=1, \ldots, N .
$$

Pour des petits déplacements ${ }^{38}$, on peut restreindre le développement au second ordre et on obtient

$$
U\left(\vec{R}_{1}, \ldots \vec{R}_{N}\right)=U_{0}+\left.\sum_{\alpha, n=1}^{3, N} \frac{\partial U}{\partial u_{\alpha}(n)}\right|_{0} u_{\alpha}(n)+\left.\frac{1}{2} \sum_{\alpha, n=1}^{3, N} \sum_{\beta, n^{\prime}=1}^{3, N} \frac{\partial^{2} U}{\partial u_{\alpha}(n) \partial u_{\beta}\left(n^{\prime}\right)}\right|_{0} u_{\alpha}(n) u_{\beta}\left(n^{\prime}\right),
$$

où $\alpha=1,2$ ou 3 se réfère aux trois directions de l'espace. Les dérivées doivent être prises par rapport aux positions d'équilibre qui, même à $T=0$, ne correspondent pas nécessairement aux minima des potentiels du fait des fluctuations quantiques. Le premier terme dans l'équation 3.10 est une constante. Le second terme contient les dérivées premières du potentiel, c'est-à-dire les forces qui agissent sur les cœurs ioniques $n$ suivant la direction $\alpha$. Ces forces doivent s'annuler à l'équilibre. Le premier terme non trivial est, par conséquent, le terme quadratique dans l'équation 3.10. Si nous traitons le problème vibratoire en nous restreignant à ce terme, nous restons dans l'approximation harmonique.

\footnotetext{
36 Dans un article récent, l'approximation adiabatique est mise en défaut dans des feuilles de graphène [19].

${ }^{37}$ Le concept qui se trouve à la base de toute notre argumentation est la théorie des bandes électroniques. C'est cette théorie qui nous permet de parler de niveaux énergétiques dans les solides. Elle conduit à décrire la fonction d'onde des électrons en terme de niveaux de particules indépendantes. Elle ne peut donc pas prendre en compte tous les effets de corrélation entre les électrons ou entre les électrons et les ions.

38 Pour que l'approximation harmonique marche, les déplacements absolus des particules peuvent en principe être assez grands pourvu que les déplacements relatifs entre atomes qui interagissent, restent petits. Pour une onde acoustique, cette condition est remplie si la longueur d'onde excède considérablement la portée des interactions. Formellement cette affirmation peut être déduite du fait que l'Hamiltonien harmonique ne dépend que des déplacements relatifs. Les vibrations des cordes de piano sont un exemple parlant pour un système classique. Ces vibrations sont harmoniques en dépit du fait que les atomes bougent de plusieurs mm ce qui est immense à l'échelle atomique - parce que la longueur d'onde de plusieurs cm est également immense comparée à la portée des interactions.
} 
Cette approximation est valable tant que les déplacements restent petits. La signification de petit dépend en réalité fortement du système étudié et de la température. Nous allons revenir sur ce point au moment où nous parlerons des déplacements moyens et des effets anharmoniques.

\subsection{Interlude : chaîne linéaire}

Avant de s'intéresser à la théorie générale, il est utile de présenter un exemple simple qui nous permettra d'introduire une bonne partie de la terminologie. Il s'agit de la chaîne linéaire. Pour éviter les problèmes liés, soit aux bords d'un système fini, soit à la normalisation d'un système infini, nous allons travailler avec un système illimité mais soumis à des conditions aux limites périodiques de type Born-Von Karman (voir la section 2.1.1). La chaîne est donc constituée de $N$ unités de base, que nous appellerons motif, le comportement de l'unité $n$ étant identique au comportement de l'unité $n+N$.

\subsubsection{Chaîne monoatomique}

Dans sa version la plus simple, les atomes sont tous identiques de masse $M$ et séparés à l'état d'équilibre mécanique par la distance $a$. Le motif de la chaîne est, dans ce cas, monoatomique et la périodicité de la chaîne de longueur $L=N a$ est décrite par $a .{ }^{39}$ On modélise les interactions entre les atomes par des forces de rappel (équivalentes à des ressorts de raideur $K$ ) souvent limitées aux premiers voisins (voir figure 14). A cause des fluctuations, la position générale d'un atome de la chaîne dépendra du temps ${ }^{40}$. Supposons qu'elle soit donnée par

$$
x(n \mid t)=x_{n}^{0}+u(n \mid t)=n a+u(n \mid t)
$$

où $x^{0}(n)=\langle x(n)\rangle=n a$ est la position d'équilibre de l'atome $n$ et $u(n \mid t)$ le déplacement par rapport à cette position. La force exercée sur la particule $j$ suite aux déplacements des particules $\{n\}$ est donnée par la somme

$$
F(n)=\sum_{n^{\prime}=1}^{N} \Phi_{n n^{\prime}}\left(u(n \mid t)-u\left(n^{\prime} \mid t\right)\right),
$$

où $\Phi_{n n^{\prime}}$ est la constante de force correspondant au ressort entre la particule $n$ et la particule $n^{\prime} .{ }^{41}$ En appliquant la loi de Newton, nous obtenons les $N$ équations de mouvement couplées du système

$$
M \frac{\partial^{2} u(n)}{\partial t^{2}}=F(n)=\sum_{n^{\prime}=1}^{N} \Phi_{n n^{\prime}}\left(u\left(n^{\prime} \mid t\right)-u(n \mid t)\right) .
$$

Ces équations peuvent s'intégrer en utilisant l'Ansatz

$$
u(n \mid t)=C e^{i\left(k x^{0}(n)-\omega t\right)}=C e^{i(k n a-\omega t)},
$$

où $C$ est, pour l'instant, une constante complexe quelconque. Les conditions aux limites périodiques impliquent que

$$
u(n \mid t)=u(n+N \mid t), \quad n=1, \ldots N
$$

\footnotetext{
39 Il faut clairement distinguer la périodicité intrinsèque de la chaîne dûe à la répétition de l'unité fondamentale, qui est la maille de longueur $a$, et la périodicité introduite de manière artificielle par nos propres soins en utilisant les conditions aux limites de type Born-Von Karman dans le but de rendre le calcul plus facile.

${ }^{40}$ Dans cette partie, nous nous attacherons à indiquer explicitement la dépendance en fonction du temps. Ce ne sera pas forcément le cas par la suite dès que cette dépendance deviendra évidente.

${ }^{41}$ Le produit $K x$ donne donc la force de rappel qu'exerce le ressort, si celui-ci est tendu (ou comprimé) d'une longueur $x$.
} 


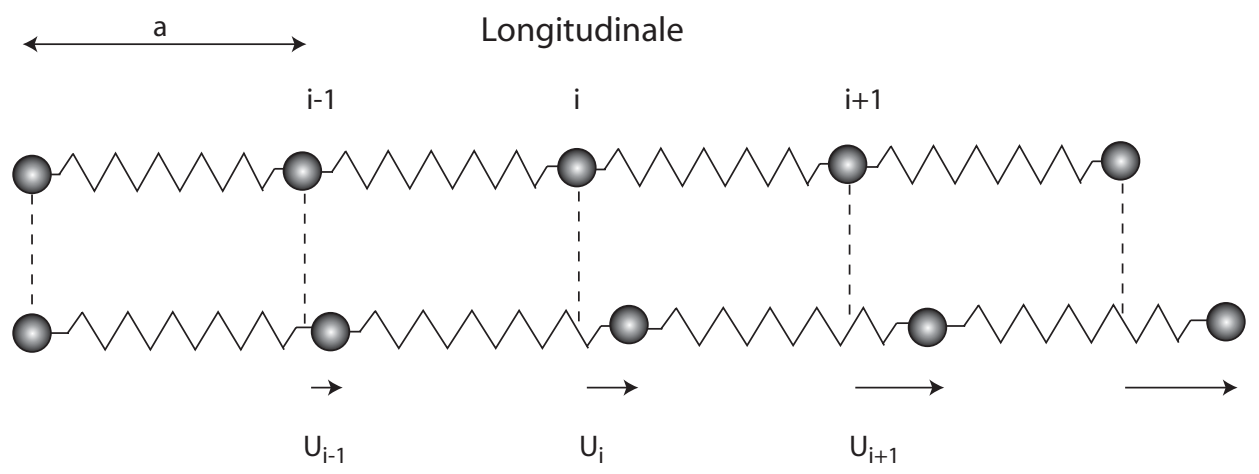

Transversale

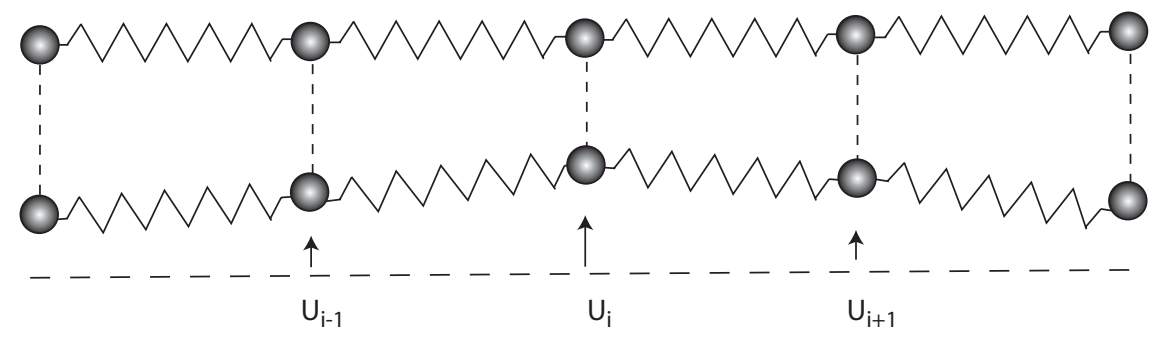

Figure 14. Couplage d'atomes par des ressorts dans une chaine monoatomique linéaire. En équilibre mécanique, les atomes sont séparés d'une distance $a$. Les forces de rappel sont proportionnelles à l'élongation des ressorts induite par les déplacements $u(n)$ des atomes. Si les mouvements atomiques sont colinéaires à la direction de la chaîne, on parlera d'oscillations longitudinales. Les "modes" transverses impliquent des déplacements atomiques perpendiculaires à la direction de la chaîne.

et donc

$$
e^{i k n a}=e^{i k(n+N) a}, \quad n=1, \ldots N
$$

Ceci n'est possible que si

$$
e^{i k N a}=\left(e^{i k a}\right)^{N}=1 .
$$

La variable $k$ ne peut donc prendre que les valeurs discrètes ${ }^{42}$

$$
k=p \frac{2 \pi}{N a}=p \frac{2 \pi}{L}, \quad p=-\frac{N}{2} \ldots-1,0,1, \ldots \frac{N}{2} .
$$

En remplaçant l'expression 3.14 dans les équations de mouvement 3.13, nous obtenons les relations suivantes, valables pour chaque valeur de la fréquence $\omega$ et pour chaque valeur de $k$

$$
M(i \omega)^{2} e^{i(k n a-\omega t)}=\sum_{n^{\prime}=1}^{N} \Phi_{n n^{\prime}}\left(e^{i\left(k n^{\prime} a-\omega t\right)}-e^{i(k n a-\omega t)}\right) .
$$

\footnotetext{
42 Pour des raisons qui deviendront évidentes plus tard, nous préférons dénombrer les valeurs de $k$ dans l'intervalle ] $-\pi / a, \pi / a$ ] au lieu de $[0,2 \pi / a[$.
} 
Nous pouvons diviser par les facteurs en commun pour obtenir

$$
-M \omega^{2}=\sum_{n^{\prime}=1}^{N} \Phi_{n n^{\prime}}\left(e^{i k\left(n^{\prime}-n\right) a}-1\right)
$$

et donc

$$
-M \omega^{2}=\sum_{n^{\prime}=1}^{N} \Phi_{\left|n^{\prime}-n\right|}\left(e^{i k\left(\left(n^{\prime}-n\right) a\right)}-1\right),
$$

où nous exploitons le fait que le système possède une symétrie de translation en introduisant la notation

$$
\Phi_{\left|n^{\prime}-n\right|} \cdot
$$

Cette symétrie implique que les quantités physiques ne peuvent pas dépendre du lieu absolu d'un atome mais seulement de la distance entre eux, et donc

$$
\Phi_{\left|n^{\prime}-n\right|}=\Phi_{1\left(1+\left|n^{\prime}-n\right|\right)}=\Phi_{n^{\prime}\left(n^{\prime}+n\right)}=\Phi_{n^{\prime}\left(n^{\prime}-n\right)}, \quad n^{\prime}, n=1, \ldots N .
$$

En décrivant le mouvement des atomes de la chaîne comme des oscillations (expression 3.14) nous avons donc réduit les $N$ équations de mouvement couplées (expression 3.13) à une seule équation qui relie, pour les $N$ valeurs du paramètre $k$, les fréquences de ces oscillations $\omega(k)$ à la masse de l'atome et aux constantes de forces entre atomes. En réaménageant l'ordre de la sommation, l'expression obtenue pour $\omega$ peut se simplifier encore davantage et on obtient comme résultat final

$$
\begin{aligned}
\omega^{2} & =-\frac{1}{M} \sum_{p>0} \Phi_{p}\left(e^{i k p a}+e^{-i k p a}-2\right) \\
& =\frac{2}{M} \sum_{p>0} \Phi_{p}(1-\cos (k p a)) \\
& =\frac{4}{M} \sum_{p>0} \Phi_{p} \sin ^{2}\left(\frac{1}{2} k p a\right)
\end{aligned}
$$

Si nous nous limitons à des ressorts entre plus proches voisins ${ }^{43}(p=1)$, décrits par une raideur $K$, nous obtenons avec $\Phi_{1}=K$ la relation ou la loi de dispersion

$$
\omega(k)=2 \sqrt{\frac{K}{M}} \sin \left(\frac{1}{2} k a\right) .
$$

Elle est illustrée sur la figure 15 où nous avons choisi le THz comme unité pour $\omega$. D'autres unités sont usuelles et nous donnons les relations entre elles :

$$
\begin{aligned}
\hbar \omega[\mathrm{Js}] & =1.055 \cdot 10^{-22} \cdot \omega[\mathrm{THz}] \\
\hbar \omega[\mathrm{meV}] & =0.658 \cdot \omega[\mathrm{THz}] \\
h v[\mathrm{meV}] & =4.137144 \cdot v[\mathrm{THz}] \\
1 \mathrm{meV} & \equiv 8.065 \mathrm{~cm}^{-1} .
\end{aligned}
$$

Si on voulait être précis, on devrait appeler $\omega$ pulsation. Nous allons suivre la pratique répandue qui consiste à utiliser le terme "fréquence" à la fois pour la pulsation $\omega=2 \pi / T$ et pour la fréquence

\footnotetext{
43 Encore appelés "premiers voisins".
} 

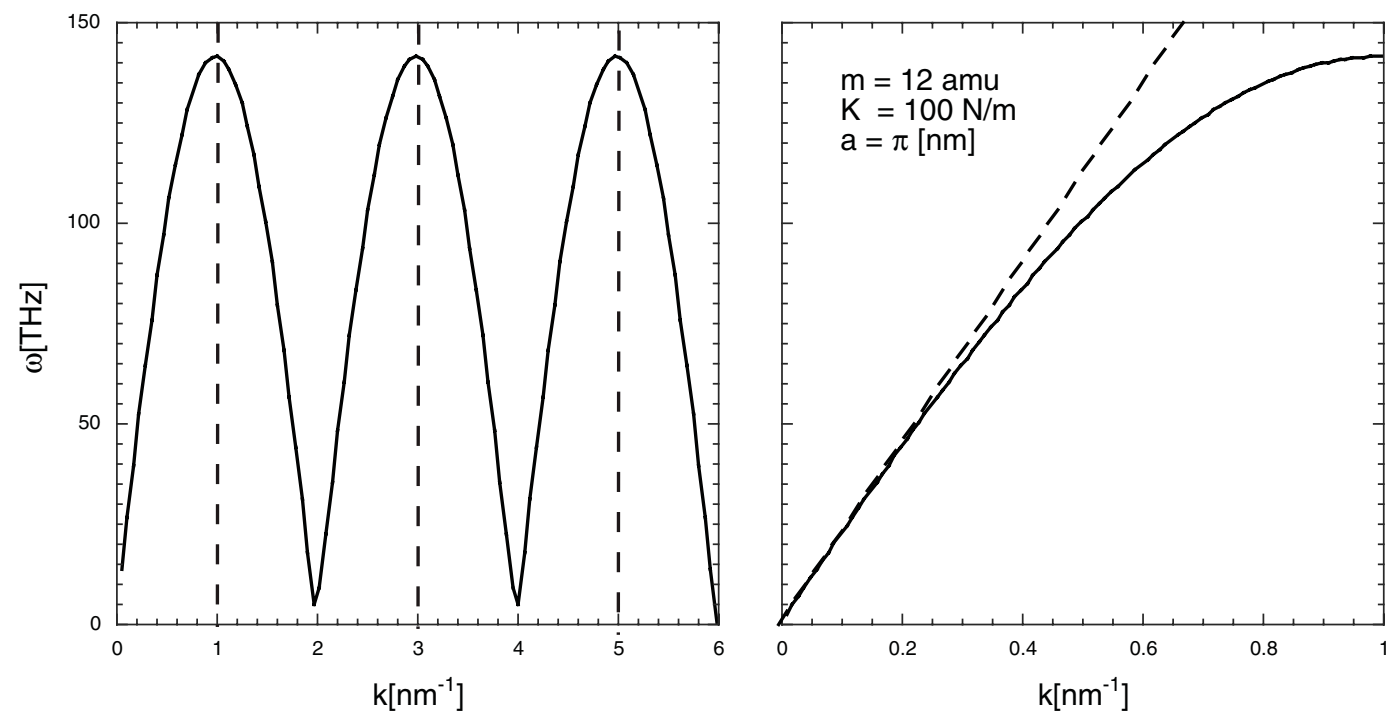

Figure 15. Courbe de dispersion $\omega(k)$ pour une chaîne linéaire. On reconnaît la périodicité. Toute l'information est donc contenue dans une seule zone de Brillouin $-\pi / a<k \leq \pi / a$. Pour $k \ll \pi / a$, la courbe est linéaire. On est alors dans le régime élastique.

$v=1 / T$ où $T$ désigne la période temporelle. Nous constatons que les relations de dispersion sont périodiques

$$
\omega(k)=\omega\left(k+\frac{2 \pi}{a}\right) .
$$

Le système possède $N$ degrés de liberté, conduisant à $N$ équations de mouvement couplées mais indépendantes. Selon la relation 3.18 nous décomptons $N$ valeurs discrètes du vecteur d'onde ${ }^{44} k$ dans un intervalle de $-\frac{\pi}{a}<k \leq \frac{\pi}{a}$. Les $N$ relations de dispersion $\omega(k)$ remplacent les $N$ équations de mouvement. Elles contiennent donc toute l'information sur la dynamique du système. L'intervalle $-\frac{\pi}{a}<k \leq \frac{\pi}{a}$ est à rapprocher à la notion de zone de Brillouin dans les cristaux. ${ }^{45}$

Dans ce contexte, il est utile de regarder les fonctions de déplacements liées aux fréquences $\omega(\vec{k})$. Ces fonctions seront forcément réelles. Elles doivent donc correspondre, soit à la partie réelle, soit à la partie imaginaire de la fonction 3.14 :

$$
\begin{aligned}
& u(n \mid t) \propto \cos (k(n a)-\omega t)=f(k, \omega) \\
& u(n \mid t) \propto \sin (k(n a)-\omega t)=f(k, \omega) .
\end{aligned}
$$

Nous constatons que $f(\omega, k)= \pm f(-\omega,-k)$, ce qui justifie le fait de n'avoir retenu que la racine positive pour $\omega(k)$. Ces fonctions de déplacement correspondent aux différentes parties (réelle et imaginaire) de la fonction d'onde d'une particule libre (voir figure 3). Ces $2 N$ fonctions forment mathématiquement une base de modes normaux dans laquelle toute forme de mouvement non-forcé de la chaîne linéaire monoatomique peut être décrite. On peut donc exprimer les déplacements des atomes

\footnotetext{
44 Même si $k$ n'est pas un vecteur dans le cas unidimensionnel, nous continuons à utiliser l'expression "vecteur d'onde" par analogie avec le cas tridimensionnel. On pourra toujours considérer $k$ comme la valeur absolue d'un vecteur $\vec{k}$ le long de la chaîne.

45 C'est à ce point qu'on se rend compte de la pertinence du choix symétrique de l'intervalle pour $k$.
} 

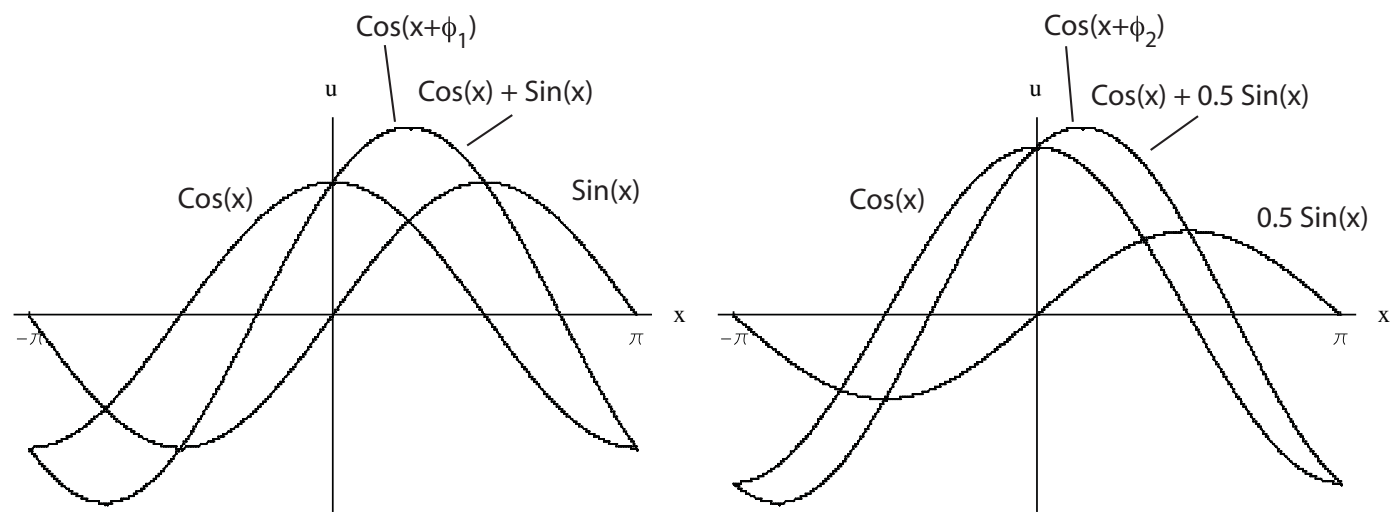

Figure 16. La figure illustre l'équivalence entre une représentation basée sur des fonctions sinus et cosinus et une représentation basée sur une amplitude et une phase. On montre deux sommes : $\cos (x)+\sin (x)$ et $\cos (x)+0.5 \sin (x)$. Dans les deux cas le résultat est un cosinus décalé par un coefficient de phase.

sous la forme

$$
u(n \mid t)=\sum_{k}\left\{a_{k} \cos (k n a-\omega t)+b_{k} \sin (k n a-\omega t)\right\}, \quad \pi / a<k \leq \pi / a, \quad n=1, \ldots N,
$$

ce qui n'est rien d'autre qu'une décomposition en série de Fourier. Les $2 N$ coefficients réels $a_{k}$ et $b_{k}$ déterminent l'état de la chaîne linéaire. Ils peuvent être exprimés à partir des positions et des vitesses des $\mathrm{N}$ atomes à un moment $t$ donné.

Une manière alternative et très intuitive d'exprimer le mouvement des atomes est donné par l'expression

$$
u(n \mid t)=\sum_{k} A_{k} \cos \left(k n a+\phi_{k}-\omega t\right), \quad \pi / a<k \leq \pi / a, \quad n=1, \ldots N .
$$

Le coefficient réel $A_{k}$ donne l'amplitude de la composante du mouvement liée au vecteur d'onde $k$.

Le déphasage entre les différentes composantes du mouvement est fixé par le coefficient réel $\phi_{k}$. Ces coefficients pilotent donc les points de retour du mouvement oscillatoire. Il fixe ainsi le temps pour lesquels les vitesses sont nulles. L'équivalence entre les deux représentations (expression 3.33 et expression 3.34) se montre facilement dans l'espace complexe. On détermine que les coefficients respectifs sont connectés via les expressions

$$
\begin{aligned}
A_{k} & =\sqrt{a_{k}^{2}+b_{k}^{2}}, \\
\phi_{k} & =2 \arctan \left(\frac{b_{k}}{A_{k}+a_{k}}\right) .
\end{aligned}
$$

La figure 16 illustre l'équivalence pour le cas simple d'un seul vecteur $k$.

Les solutions de forme $\cos (k n a-(\omega t+\phi))$ décrivent des ondes dites progressives. Les nœuds se déplacent avec le temps à une vitesse, appelée vitesse de phase, donnée par l'expression

$$
V_{\mathrm{ph}}=\frac{\omega}{k}=\frac{\lambda}{T}
$$

avec $T$ la périodicité et $\lambda$ la longueur d'onde du mouvement oscillatoire.

Comme pour les particules libres, nous pouvons former des ondes stationnaires en exploitant le fait que $\omega(k)$ est une fonction paire, c'est-à-dire $\omega(k)=\omega(-k)$. Les ondes stationnaires forment une 
autre base dans laquelle nous pouvons décrire le mouvement de la chaîne. Nous obtenons la moitié des fonctions de cette base à partir des parties réelles des sommes et différences des fonctions de base originales (expression 3.33).

$$
\begin{aligned}
u_{\text {stat. }}^{\text {paire,paire }}(n \mid t) & \propto \operatorname{Re}[\exp (i(k n a-\omega t))+\exp (-i(k n a-\omega t))] \\
& =\operatorname{Re}[\exp (-i \omega t)(\exp (i k n a)+\exp (-i k n a))] \\
& =2 \operatorname{Re}[\exp (-i \omega t) \cos (k n a)] \\
& \propto \cos (\omega t) \cos (k n a), \quad 0 \leq k \leq \pi / a . \\
u_{\text {stat. }}^{\text {impaire,impaire }}(n \mid t) & \propto \operatorname{Re}[\exp (i(k n a-\omega t))-\exp (-i(k n a-\omega t))] \\
& =\operatorname{Re}[\exp (-i \omega t)(\exp (i k n a)-\exp (-i k n a))] \\
& =\operatorname{Re}[2 i \exp (-i \omega t) \sin (k n a)] \\
& \propto \sin (\omega t) \sin (k n a), \quad 0 \leq k \leq \pi / a .
\end{aligned}
$$

Les $N$ fonctions de base manquantes sont données par les parties imaginaires.

$$
\begin{aligned}
u_{\text {stat. }}^{\text {paire, impaire }}(n \mid t) & \propto \operatorname{Im}[\exp (i(k n a-\omega t))+\exp (-i(k n a-\omega t))] \\
& \propto \sin (\omega t) \cos (k n a), \quad 0<k \leq \pi / a . \\
u_{\text {stat. }}^{\text {impaire,paire }}(n \mid t) & \propto \operatorname{Im}[\exp (i(k n a-\omega t))-\exp (-i(k n a-\omega t))] \\
& \propto \cos (\omega t) \sin (k n a), \quad 0<k \leq \pi / a .
\end{aligned}
$$

Ces fonctions stationnaires sont caractérisées par leur parité par rapport à l'inversion de la direction et du temps. Les ondes sont qualifiées de "stationnaires" car leurs nœuds sont invariants dans le temps. En pratique des ondes stationnaires peuvent être obtenues en réfléchissant des ondes progressives aux bords du système. Nous rappelons qu'une réflexion sur un bord rigide (de masse infinie) produit un déphasage ${ }^{46}$ de $\pi$, tandis que la réflexion sur un bord souple (masse égale à zéro) ne produit pas de déphasage.

Le fait que les courbes de dispersion soient périodiques en $k$ implique l'existence d'une valeur maximale pour $\omega(k)$. La largeur de bande $\Delta \omega=\omega_{\max }-\omega_{\min }$ des excitations est donc finie. La valeur minimale est toujours zéro pour $k=0$. Ceci peut être imputé à la symétrie de translation de la chaîne et reflète le fait que le déplacement de l'ensemble de la chaîne, en absence de champ macroscopique extérieur, ne rencontre aucune résistance et aucune force de rappel.

Dans l'exemple traité, le mouvement des atomes était contraint le long de la direction de la chaîne, c'est-à-dire le long du vecteur d'onde $k$. Nous parlons d'ondes longitudinales. Nous pouvons aussi imaginer un système où les atomes ne peuvent bouger que sur des rails perpendiculaires à la direction de la chaîne. Nous obtiendrons des résultats assez similaires à ceux obtenus pour la chaîne unidimensionnelle, hormis le fait que les mouvements seraient perpendiculaires à la direction de modulation, c'est-à-dire au vecteur d'onde $k$. On parlera de modes transverses. Ce sont les modes normaux, illustrés par exemple par la vibration des cordes d'un piano.

Même si les interactions dans les solides sont normalement maximales entre voisins directs, les interactions entre atomes plus éloignés jouent un rôle important. Cela vaut par exemple pour les solides ioniques, étant donné que les forces de Coulomb décroissent relativement lentement avec la distance. Il est donc intéressant de regarder l'influence de telles interactions sur la courbe de dispersion de notre chaîne. L'expression 3.24 indique que les interactions entre voisins plus éloignés contribuent à $\omega^{2}$ par

\footnotetext{
46 C'est le cas pour un piano.
} 

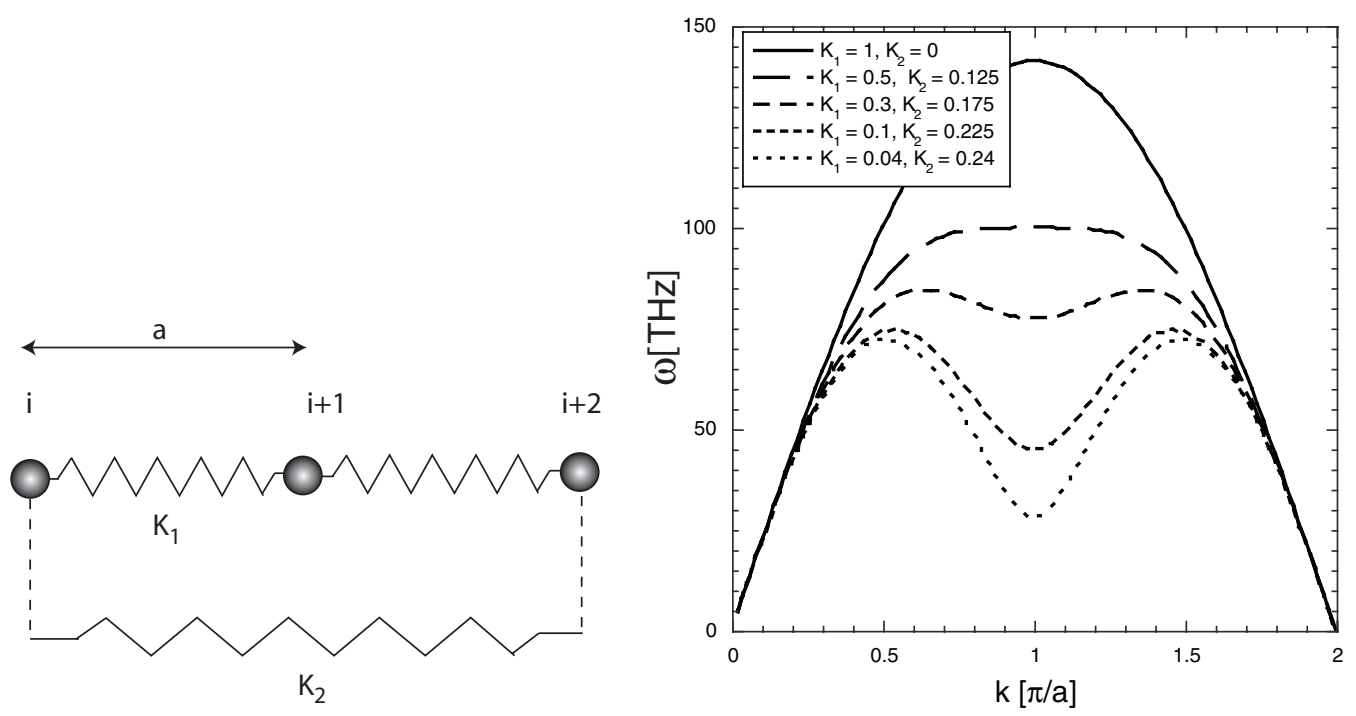

Figure 17. Courbes de dispersion pour une chaîne monoatomique avec interaction entre premiers et seconds voisins pour une série de constantes de force $\left(K_{1}, K_{2}\right)$. Le rapport $K_{2} / K_{1}$ varie de 0 à 6 .

l'addition de termes en

$$
\sin ^{2}\left(\frac{1}{2} k(p a)\right), \quad p=2,3,4 \ldots,
$$

qui sont des modulations de la contribution fondamentale

$$
\sin ^{2}\left(\frac{1}{2} k a\right) .
$$

Ces contributions auront donc tendance à structurer la courbe de dispersion. Nous présentons dans la figure 17 un exemple concret illustrant deux constantes de forces différentes entre premiers et seconds voisins. Pour rendre la comparaison significative, nous avons choisi $K_{1}$ et $K_{2}$ tels qu'à $k \ll \pi / a$, la courbe de dispersion ait un comportement identique à celui de la chaîne présentée en figure 15 . On trouvera les bons rapports $K_{1} / K_{2}$ en utilisant l'approximation

$$
\frac{\omega^{2}}{(k a)^{2}}=\sum_{p>0} p^{2} \frac{K_{p}}{M}, \quad \text { pour } \quad k \ll \frac{\pi}{a} .
$$

On constate que l'interaction entre les particules $n$ et $n+2$ tasse la courbe de dispersion en bordure de zone. A partir d'un certain $K_{2}$, la courbe développe même un minimum en bord de zone. On dit que le mode en bord de zone devient mou. Pour des valeurs de $K_{1}$ tendant vers zéro, une particule donnée ne sentira plus la présence de ses voisins les plus proches. Elle aura l'impression de faire partie d'un système d'atomes séparés par une distance de $2 a$ et reliés entre eux par des ressorts de raideur $K_{2}$. Un tel système possède une maille deux fois plus grande et donc une zone de Brillouin avec une périodicité de $\pi / 2 a$. Le fait que $\omega(\pi / a)=0$ reflète que les déplacements des atomes pour les modes en bord de zone -où alternativement un atome sur deux oscille en phase et se rapproche de ses voisins directs- ne rencontrent plus aucune force de rappel. Si cela se produit dans un système réel, on assistera à une transition de phase. 


\subsubsection{Chaîne linéaire diatomique}

Rajoutons un peu plus de complexité au modèle de la chaîne linéaire en peuplant chaque site du réseau alternativement avec des atomes de masses $M_{1}$ et $M_{2}$ différentes. Le paramètre de maille $a$ de la chaîne passe ainsi à deux fois la distance entre voisins, même si le couplage reste limité aux interactions entre voisins directs.

En partant de l'expression 3.12 nous obtenons le jeu d'équations couplées du mouvement

$$
\begin{aligned}
M_{1} \ddot{u}(1, l \mid t) & =K[u(2, l \mid t)-u(1, l \mid t)]+K[u(2, l-1) \mid t)-u(1, l \mid t)] \\
& =-K[2 u(1, l \mid t)-u(2, l \mid t)-u(2, l-1 \mid t)], \\
M_{2} \ddot{u}(2, l \mid t) & =K[u(1, l \mid t)-u(2, l \mid t)]+K[u(1, l+1 \mid t)-u(2, l)] \\
& =-K[2 u(2, l)-u(1, l)-u(1, l+1)],
\end{aligned}
$$

où $u(1, l \mid t)$ et $u(2, l \mid t)$ représentent les déplacements des atomes de masse $M_{1}$ et $M_{2}$ dans la maille $l$ au moment $t$, respectivement. Ces équations peuvent être intégrées avec l'Ansatz

$$
\begin{aligned}
& u(1, l \mid t)=u(1) e^{i(k(l a)-\omega t)} \\
& u(2, l \mid t)=u(2) e^{i(k(l a)-\omega t)} .
\end{aligned}
$$

Si nous remplaçons ces expressions dans les équations 3.46 et simplifions par le facteur commun $e^{i(k l a-\omega t)}$, nous obtenons deux équations homogènes couplées

$$
\begin{aligned}
& -M_{1} \omega^{2} u(1)=-K\left(2 u(1)-u(2)\left(1+e^{-i k a}\right)\right) \\
& -M_{2} \omega^{2} u(2)=-K\left(2 u(2)-u(1)\left(1+e^{i k a}\right)\right),
\end{aligned}
$$

qui sont indépendantes de l'indice de maille $l$. Il est pratique et instructif pour l'étude du cas général d'écrire ces équations sous forme matricielle

$$
\omega^{2} \vec{e}=\mathbf{D} \vec{e}
$$

avec les vecteurs propres

$$
\vec{e}=\left(\begin{array}{l}
\sqrt{M_{1}} u(1) \\
\sqrt{M_{2}} u(2)
\end{array}\right)
$$

et la matrice appelée matrice dynamique définie $\operatorname{par}^{47}$

$$
\mathbf{D}(k)=\left(\begin{array}{cc}
\frac{2 K}{M_{1}} & -\frac{K}{\sqrt{M_{1} M_{2}}}\left(1+e^{-i k a}\right) \\
-\frac{K}{\sqrt{M_{1} M_{2}}}\left(1+e^{i k a}\right) & \frac{2 K}{M_{2}}
\end{array}\right) .
$$

On obtiendra une solution pourvu que le déterminant de

$$
\mathbf{D}-\omega^{2} \mathbf{1}
$$

s'annule, c'est-à-dire

$$
\left|\mathbf{D}-\omega^{2} \mathbf{1}\right|=0 \text {. }
$$

\footnotetext{
47 Il faut préciser ici qu'il s'agit de la matrice dynamique dans l'espace réciproque, c'est-à-dire $\mathbf{D}(k)$ est une fonction du vecteur d'onde $k$. Comme nous verrons dans la partie générale, il est aussi possible de définir une matrice dynamique dans l'espace direct. Les deux représentations sont reliées par des transformées de Fourier.
} 

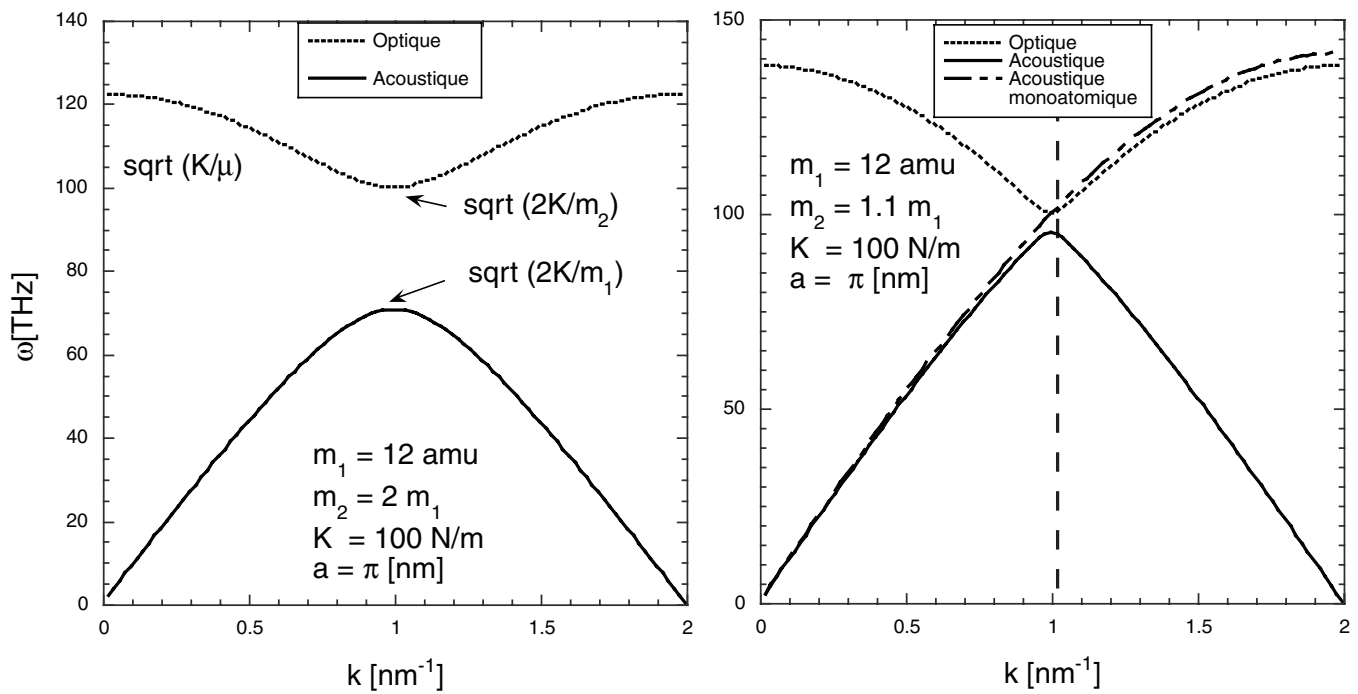

Figure 18. Courbes de dispersion $\omega(k)$ pour une chaine linéaire diatomique. En dehors de la branche acoustique de basse fréquence, on obtient une branche optique de haute fréquence. Les déplacements en centre de zone et en bord de zone sont schématisés sur la figure 21. En comparant les courbes pour deux valeurs différentes du rapport $M_{1} / M_{2}$, nous voudrions montrer comment la séparation des branches optiques et acoustiques dépend de la différence de masse. Dans la limite de $M_{1} \rightarrow M_{2}$, nous retrouvons la courbe de dispersion de la chaîne monoatomique, mais repliée dans la zone de Brillouin de la chaîne diatomique qui est deux fois moins étendue que celle de la chaîne monoatomique. La courbe pour une chaîne monoatomique de masse $M_{1}$ est sur la figure de droite. Le bord de zone fait office de miroir dans cette opération de repliement.

Cette condition est vérifiée, si les fréquences $\omega$ sont liées aux vecteurs d'onde $k$ par la relation

$$
\omega_{ \pm}^{2}(k)=\frac{K}{M_{1} M_{2}}\left(M_{1}+M_{2} \pm \sqrt{M_{1}^{2}+M_{2}^{2}+2 M_{1} M_{2} \cos k a}\right) .
$$

Les valeurs $\omega_{ \pm}^{2}(k)$ correspondent aux valeurs propres de la matrice dynamique. Pour un vecteur d'onde $k$ donné, il existe donc deux excitations notées + et - dans la suite de cette section. Les courbes $\omega_{+}(k)$ et $\omega_{-}(k)$ sont appelées branches de dispersion. Elles sont tracées sur la figure 18 pour un cas spécifique. Les vecteurs propres du mode $j$ seront notés $\vec{e}_{j}(k)$ et les composantes $e_{j}(k \mid n)$ de ce vecteur représentent les vibrations des atomes $n$ suivant l'axe de la chaîne pour le mode $j$, pondérées de la racine carrée de leur masse $M_{n}$. Dans le cas qui nous intéresse ici, les deux vecteurs propres seront notés

$$
\vec{e}_{ \pm}(k)=\left(\begin{array}{c}
\sqrt{M_{1}} e_{ \pm}(k \mid 1) \\
\sqrt{M_{2}} e_{ \pm}(k \mid 2)
\end{array}\right) .
$$

Comme pour n'importe quel vecteur propre, l'amplitude n'est pas figée, c'est-à-dire que si

$$
\vec{D}_{ \pm}(k)=\omega_{ \pm}^{2}(k) \vec{e}_{ \pm}(k),
$$

il suit également que

$$
\mathbf{D}\left(\lambda \vec{e}_{ \pm}(k)\right)=\omega_{ \pm}^{2}(k)\left(\lambda \vec{e}_{ \pm}(k)\right),
$$

avec $\lambda=A \exp (i \phi)$ un nombre complexe quelconque. Pour cette raison, nous pouvons toujours choisir un vecteur propre normalisé. La quantité pertinente est donc le rapport entre les amplitudes $|u(1)|$ et $|u(2)|$ et non pas les amplitudes absolues. Ceci est la traduction mathématique de l'indépendance des fréquences de vibration par rapport aux amplitudes d'oscillation en régime harmonique. Ces amplitudes 
ne sont donc pas déterminées par les équations de mouvement mais résultent purement des conditions initiales. La même remarque vaut pour une phase globale $\phi$.

Posons

$$
\vec{u}=\left(\begin{array}{l}
u(1) \\
u(2)
\end{array}\right)
$$

et choisissons $u(1)$ tel que

$$
u(1)=\alpha u(2),
$$

de telle sorte que le vecteur $\vec{u}$ soit normalisé ${ }^{48}$. Dans ce cas, le vecteur de déplacement s'écrit tout simplement comme

$$
\vec{u}=\left(\begin{array}{c}
\frac{\alpha}{\sqrt{1+\alpha^{2}}} \\
\frac{1}{\sqrt{1+\alpha^{2}}}
\end{array}\right) .
$$

Remplaçant $\vec{u}$ dans les équations 3.48 , nous obtenons

$$
\begin{aligned}
& \alpha=-\frac{K(1+\exp (-i k a))}{\left(M_{1} \omega^{2}-2 K\right)} \\
& \alpha=-\frac{\left(M_{2} \omega^{2}-2 K\right)}{K(1+\exp (i k a))} .
\end{aligned}
$$

Les deux équations sont complètement dépendantes à cause de la relation 3.54, c'est-à-dire qu'elles contiennent les mêmes informations.

Pour un vecteur d'onde $k$ donné, on obtient donc pour chaque branche de dispersion, une relation fixe entre les mouvements des deux atomes de la maille primitive. Nous constatons que le facteur $\alpha$, reliant le déplacement de la particule de masse $M_{2}$ à celui de la particule de masse $M_{1}$, est complexe Nous pouvons ainsi écrire ce facteur comme

$$
\alpha(k)=\alpha=|\alpha| \exp (i \Phi) .
$$

Avec cette notation nous obtenons à partir des équations 3.47 l'expression suivante pour les déplacements des atomes:

$$
\begin{aligned}
& u(1, l \mid t)=\left(|\alpha| / \sqrt{|\alpha|^{2}+1}\right) e^{i(k l a-\omega t+\Phi)}, \\
& u(2, l \mid t)=\left(1 / \sqrt{|\alpha|^{2}+1}\right) e^{i(k l a-\omega t)} .
\end{aligned}
$$

En ne retenant que la partie réelle ces expressions permettent d'écrire

$$
\begin{aligned}
& u(1, l \mid t)=\left(|\alpha| / \sqrt{|\alpha|^{2}+1}\right) \cos (k l a-\omega t+\Phi), \\
& u(2, l \mid t)=\left(1 / \sqrt{|\alpha|^{2}+1}\right) \cos (k l a-\omega t) .
\end{aligned}
$$

On voit clairement que les déplacements des deux atomes ne sont, en général, pas en phase les uns avec les autres. ${ }^{49}$ Le mouvement d'une particule est décalé par rapport à l'autre et ce décalage dépend

48 Ceci est possible pourvu que la particule de masse $M_{2}$ n'est pas à l'arrêt pour les modes de la branche de dispersion concernée. Si tel est le cas il faut choisir $u(2)=\alpha u(1)$. Ce choix ne change rien aux arguments présentés.

${ }^{49}$ En superposant, pour une branche donnée, les modes de vecteur d'onde $k$ avec ceux de vecteur d'onde $-k$ et en exploitant le fait que $\alpha(k)=\alpha^{*}(-k)$ nous pouvons décrire des modes stationnaires (voir expression 3.39). En nous limitant à la partie paire nous obtenons comme solution possible

$$
\begin{aligned}
& \left.u(1, l \mid t)=2\left(|\alpha| / \sqrt{2} \sqrt{|\alpha|^{2}+1}\right) \cos (k l a+\phi)\right) \cos (\omega t), \\
& u(2, l \mid t)=-2\left(1 / \sqrt{2} \sqrt{|\alpha|^{2}+1}\right) \cos (k l a) \cos (\omega t) .
\end{aligned}
$$




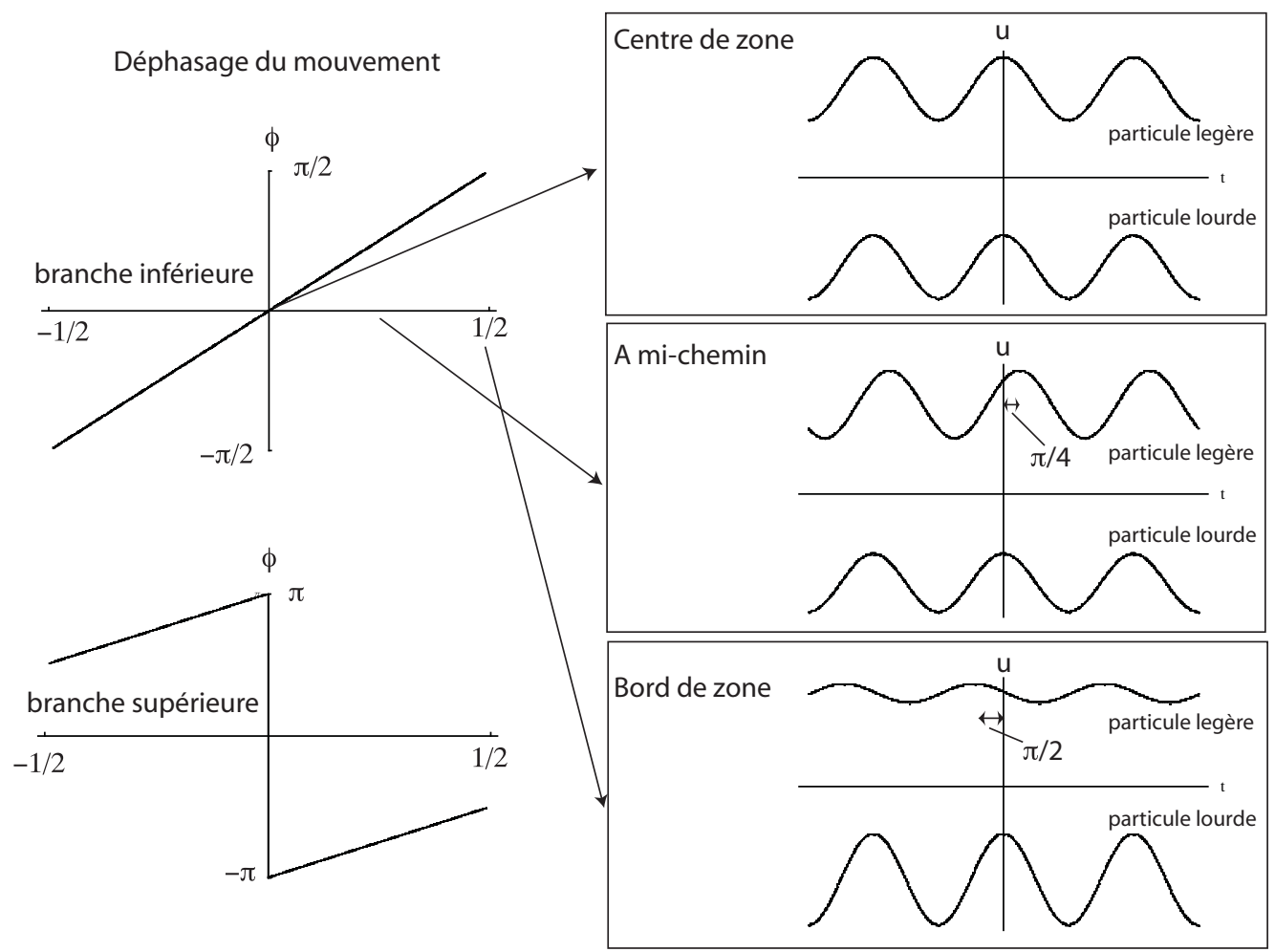

Figure 19. Chaîne linéaire avec un motif à deux atomes dont les masses ont un rapport $1 / 2$. Nous montrons le déphasage du mouvement des deux atomes en fonction du vecteur d'onde $k$. Le déphasage exprimé par l'argument $\phi$ du nombre complexe $\alpha$ (éq. 3.60) est linéaire en $k$ et croît pour la branche basse de $-\pi / 2$ à $\pi / 2$ en passant de la limite inférieure à la limite supérieure de la zone de Brillouin. Pour la branche supérieure, on constate qu'en centre de zone le mouvement des deux particules est en anti-phase $(\phi= \pm \pi)$. La différence de phase diminue en s'éloignant du centre vers les bords. Les schémas à droite indiquent les oscillations des deux particules en fonction du temps pour trois points spécifiques de la courbe de dispersion inférieure. Les mouvements des deux particules sont en phase et d'amplitude identique en centre de zone. Dès qu'on s'éloigne du centre de zone, le mouvement de la particule légère est décalé par rapport à celui de la particule lourde. En même temps, l'amplitude de la particule légère décroît au profit de celle de la particule lourde.

de k. On peut facilement déduire de l'équation 3.59 que le déphasage $\phi(k)$ est une fonction linéaire de $k$. Le vecteur propre complexe indique à la fois le rapport des amplitudes ainsi que le déphasage du mouvement d'une particule par rapport aux autres (voir la figure 19 pour quelques valeurs de $k$ ). La figure 20 montre les amplitudes de déplacement en fonction de $k$ pour deux chaînes diatomiques concrètes.

Regardons maintenant quelques points spécifiques le long des courbes de dispersion.

- Centre de zone. Soit $k \ll \pi / a$.

Dans ce cas

$$
\cos (k a) \approx 1-\frac{(k a)^{2}}{2}
$$

On voit que les deux expressions ont la forme classique d'une onde stationnaire. Néanmoins il est important de constater que les deux particules ne partagent pas les mêmes nœuds. Ceci n'est pas étonnant si on considère que le mouvement des deux particules est en général déphasé. 

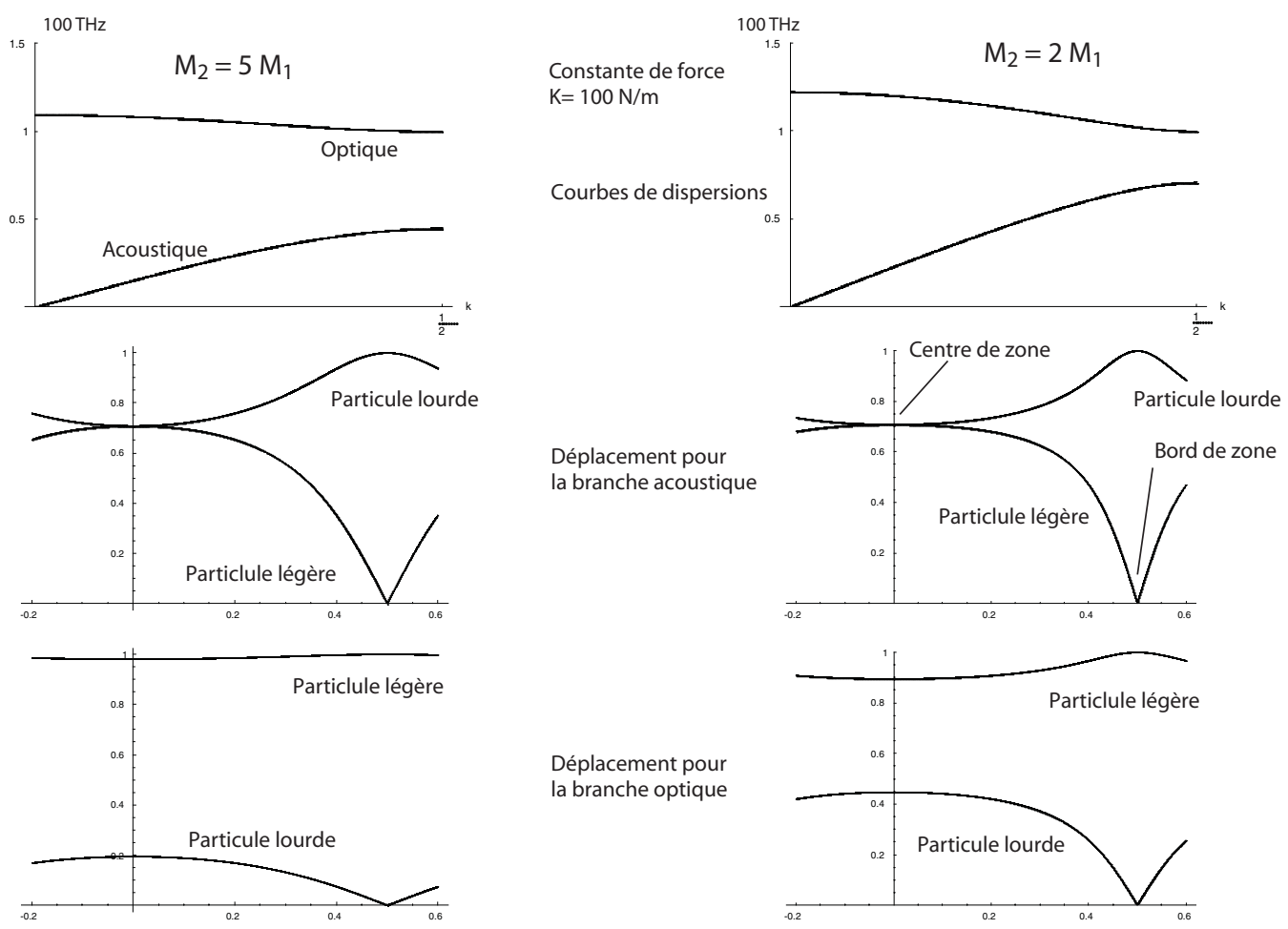

Figure 20. Chaîne linéaire avec un motif à deux atomes de masses différentes. Nous montrons les amplitudes de déplacement des deux atomes en fonction du vecteur d'onde $k$ pour les deux branches de dispersion selon éq. 3.61. Pour la branche inférieure dite acoustique, les deux atomes bougent avec la même amplitude en centre de zone. En s'éloignant du centre, la particule légère perd de l'amplitude pour s'arrêter complètement en bord de zone. Ce processus de perte d'amplitude est d'autant plus prononcé que le rapport entre les masses est élevé. Pour la branche supérieure dite "optique", les particules se déplacent en centre de zone de manière à ce que le centre de gravité du motif reste préservé. Ceci implique que l'amplitude de la particule légère devient très grande pour des rapports de masse très importants. Pour cette branche, c'est la particule lourde qui s'arrête en bord de zone.

Avec cette approximation, éq. 3.54 donne :

$$
\begin{aligned}
& \omega_{-}(k) \approx \sqrt{\frac{K}{2\left(M_{1}+M_{2}\right)}|k a|} \\
& \omega_{+}(k) \approx \sqrt{\frac{2 K}{\mu}},
\end{aligned}
$$

où $\mu=M_{1} M_{2} /\left(M_{1}+M_{2}\right)$ est la masse reduite des deux particules de la maille de la chaîne. La fréquence de la branche acoustique $\omega_{-}(k)$ est donc nulle à $k=0$ et augmente linéairement avec le vecteur d'onde. C'est la situation que nous connaissons pour la lumière et pour les ondes sonores de grande longueur d'onde. Pour le vecteur propre, on obtient selon éq. 3.59

$$
\alpha_{-}=\frac{u(1)}{u(2)}=1 \quad \text { pour } \quad k \rightarrow 0
$$




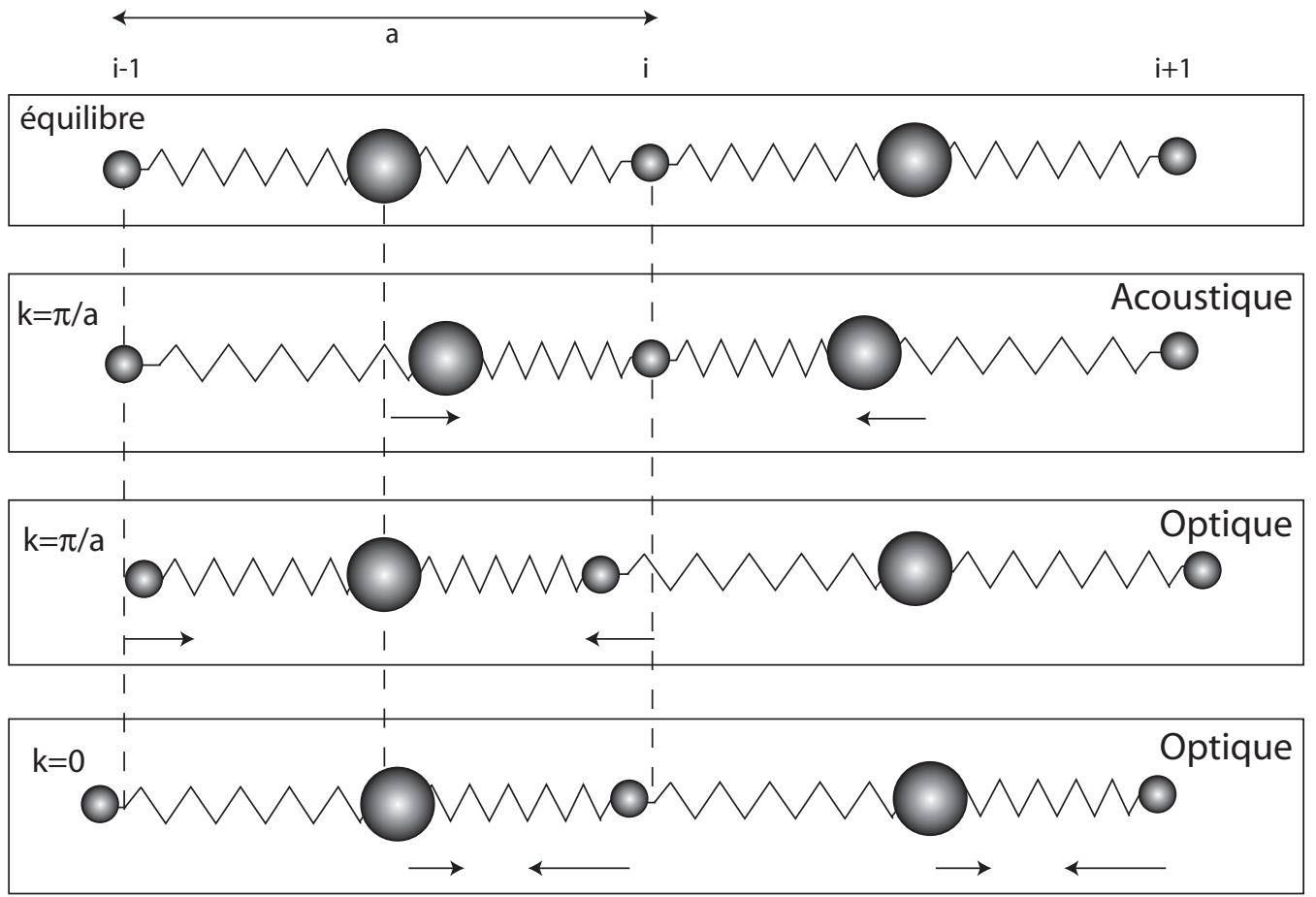

Figure 21. Chaîne linéaire avec un motif à deux atomes de masses différentes. Schéma de déplacement pour le mode optique à $k=0$ et pour le mode acoustique (oscillation uniquement de l'atome lourd) et optique (oscillation uniquement de l'atome léger) à $k=\pi / a$.

Les deux sous-réseaux se déplacent donc en phase. C'est le comportement attendu pour une onde sonore pour laquelle

$$
\omega=c k,
$$

avec $c$ la vitesse du son. C'est la raison pour laquelle la branche de dispersion basse est appelée "branche acoustique". Il est important de faire remarquer qu'en dépit du fait que les déplacements sont identiques, les deux composantes du vecteur propre $e_{-}(k)$ se distinguent :

$$
\frac{e_{-}(k \mid 1)}{e_{-}(k \mid 2)}=\frac{\sqrt{M_{1}} u(1)}{\sqrt{M_{2}} u(2)}=\sqrt{\frac{M_{1}}{M_{2}}} \text { pour } k \rightarrow 0 .
$$

Si on construit un paquet d'onde avec des excitations acoustiques, ce paquet va se propager avec la vitesse de groupe (éq. 2.58)

$$
v_{g}=\left(\frac{\partial \omega(k)}{\partial k}\right)_{k \rightarrow 0}=a \sqrt{\frac{K}{2\left(M_{1}+M_{2}\right)}}=c .
$$

En s'éloignant de $k=0$, la branche acoustique tombe en-dessous de la ligne $\omega=c k$. La vitesse de groupe diminue pour tomber à zéro en bord de zone. 
Pour la branche optique de haute fréquence $\omega_{+}(k)$, les composantes du vecteur propre sont reliées par la condition

$$
\alpha_{+}=\frac{u(1)}{u(2)}=-\frac{M_{2}}{M_{1}}
$$

Une particule de masse $M_{1}$ se déplace donc en opposition de phase par rapport à sa voisine de masse $M_{2}$. Le déplacement du centre de masse (noté c.m) des deux atomes par maille est donné par

$$
u_{\mathrm{c} . \mathrm{m} .}=M_{1} u(1)+M_{2} u(2)=0
$$

est nulle. Le centre de masse reste donc immobile comme ce qui est attendu pour une vibration locale. Ces vibrations peuvent interagir avec la lumière, d'où leur appellation de branche optique.

- Bord de zone. Soit $k=\pi / a$

Nous obtenons pour les deux fréquences

$$
\begin{aligned}
& \omega_{-}\left(\frac{\pi}{a}\right)=\sqrt{\frac{2 K}{M_{1}}}, \\
& \omega_{+}\left(\frac{\pi}{a}\right)=\sqrt{\frac{2 K}{M_{2}}} .
\end{aligned}
$$

Les vecteurs propres associés sont caractérisés par

$$
\begin{aligned}
& \alpha_{-}=0, \\
& \alpha_{+}=\infty .
\end{aligned}
$$

Nous sommes confrontés à une situation où le sous-réseau des particules d'un certain type (disons celles de masse $M_{1}$ ) oscille, tandis que le réseau des particules restantes (donc de masse $M_{2}$ ) est au repos. La phase change de $180^{\circ}$ d'une maille à l'autre. Le réseau des particules plus lourdes oscille à la fréquence la plus basse. Entre $\omega_{-}$et $\omega_{+}$, il y a une région de fréquence dans laquelle on ne trouve aucune excitation. Ce gap est donné par la différence de masse entre les deux sousensembles. Si une des deux masses, disons $M_{2}$, est très petite par rapport à l'autre, elle va dominer l'inverse de la masse réduite

$$
\mu^{-1}=\frac{1}{M_{1}}+\frac{1}{M_{2}} \approx \frac{1}{M_{2}} \text { pour } \quad M_{2} \ll M_{1} .
$$

Dans ce cas, les fréquences de la branche optique à $k=0$ et à $k=\pi / a$ sont presque identiques (voir éq. 3.67 et 3.75). La courbe de dispersion optique est plate. La vitesse de groupe (éq. 2.58) est par conséquent nulle. En utilisant une large gamme de vecteur d'onde on pourrait construire des paquets d'ondes très localisés et qui ne s' étaleraient que très peu (voir l'éq. 2.47).

\subsubsection{Chaîne linéaire à ressorts alternés}

On peut créer un motif dans la chaîne linéaire en introduisant des différences de masses -comme on vient de voir dans la section précédente- ou de constantes de force, qui est le cas qui nous intéresse dans cette partie. Les systèmes ainsi obtenus ne sont pas complètement isomorphes du fait que la masse joue un rôle pour la dynamique que les constantes de forces ne jouent pas. Par conséquent, il est utile de comparer les deux cas de figure. Que la chaîne à constantes de forces alternées puisse constituer 
aussi le modèle le plus simple pour un cristal moléculaire peut servir de motivation supplémentaire. ${ }^{50}$ La molécule est associée aux paires d'atomes liés par la constante de force la plus forte. L'interaction entre les molécules est prise en compte par des ressorts plus faibles. Nous n'allons pas répéter le calcul détaillé pour la chaîne à masses alternées. Nous allons plutôt nous contenter de fournir les résultats principaux, là où ils apportent des informations nouvelles.

Soit $f$ et $F$ les raideurs des deux ressorts qui lient alternativement les atomes d'une chaîne linéaire de masse unique $M$ (voir figure 22). Nous choisissons $f<F$ par convention. Utilisant l'Ansatz 3.47 nous obtenons les équations :

$$
\begin{aligned}
& -M \omega^{2} u(1)=-(f+F) u(1)+\left(F+f e^{-i k a}\right) u(2), \\
& -M \omega^{2} u(2)=-(f+F) u(2)+\left(F+f e^{i k a}\right) u(1) .
\end{aligned}
$$

Ce qui donne la matrice dynamique

$$
\mathbf{D}(k)=\frac{1}{M}\left(\begin{array}{cc}
f+F & -\left(F+f e^{-i k a}\right) \\
-\left(F+f e^{i k a}\right) & f+F
\end{array}\right) .
$$

Les deux courbes de dispersion, valeurs propres de $\mathbf{D}$, sont données par l'expression

$$
M \omega_{ \pm}^{2}=(f+F) \pm \sqrt{f^{2}+F^{2}+2 f F \cos k a} .
$$

avec les vecteurs propres

$$
\alpha \pm=\frac{e_{ \pm}(k \mid 2)}{e_{ \pm}(k \mid 1)}=\mp \frac{F+f e^{-i k a}}{\left|F+f e^{i k a}\right|} .
$$

Ces fonctions ressemblent à celles que nous avions trouvées pour la chaîne biatomique (éq. 3.54 et 3.59). Il y a néanmoins deux différences importantes : 1) comme dans le cas de la chaîne mono-atomique, la masse $M$ reste un simple facteur multiplicatif du carré de la fréquence et 2) les vecteurs propres ne dépendent pas des masses. Ces faits mathématiques soulignent les rôles différents que jouent les constantes de force et la masse, respectivement.

Le fait que ni la masse, ni les fréquences $\omega$, n'entrent dans l'expression 3.82, facilite l'interprétation par rapport à l'expression analogue pour la chaîne à deux atomes de masses différentes. Nous constatons tout d'abord que $\alpha$ est toujours normalisé. Les deux atomes ont par conséquent toujours la même amplitude de vibration.

Ce résultat n'est pas étonnant si on considère que les deux atomes sont reliés par une symétrie supplémentaire du système et doivent, de ce fait, être considerés équivalents. Il est instructif de s'attarder un moment sur cet aspect. C'est en effet le cas le plus simple pour montrer comment des arguments de symétrie placent des contraintes aux vecteurs propres d'un système de dynamique du réseau. L'opération de symétrie dont il s'agit ici est l'inversion par rapport à l'origine, que nous plaçons au milieu des deux atomes. $^{51}$

Les deux atomes ont donc le même environnement à la seule différence que l'un possède un ressort fort à droite et l'autre un ressort fort à gauche.

Le système est donc invariant par application de l'inversion I, ce qui veut dire qu'un observateur ne pourrait pas distinguer le système avant application de $I$ du sytème après.

\footnotetext{
50 Dans la réalité, les atomes qui forment une molécule sont beaucoup plus proches les uns des autres que les molécules ellesmêmes. Nous devrions par conséquent construire une chaîne avec deux atomes par maille primitive, de positions à l'équilibre $n a$ et $n a+d$, avec $d<a / 2$. C'est cette différence de distance qui est à l'origine de la différence des constantes de forces. Dans l'approximation harmonique les distances d'équilibre n'apparaissent pas explicitement dans l'expression de l'énergie. Ce qui compte est uniquement le champ des déplacements. Nous pouvons donc nous affranchir de cette complication supplémentaire étant donné qu'elle n'a pas de conséquence pour les résultats présentés. Nous présumons donc implicitement que les distances entre particules sont toutes identiques.

51 Dans le cas unidimensionnel, l'inversion équivaut à une réflexion par rapport à un plan perpendiculaire à la direction de la chaîne et qui coupe la distance entre les deux atomes en deux.
} 


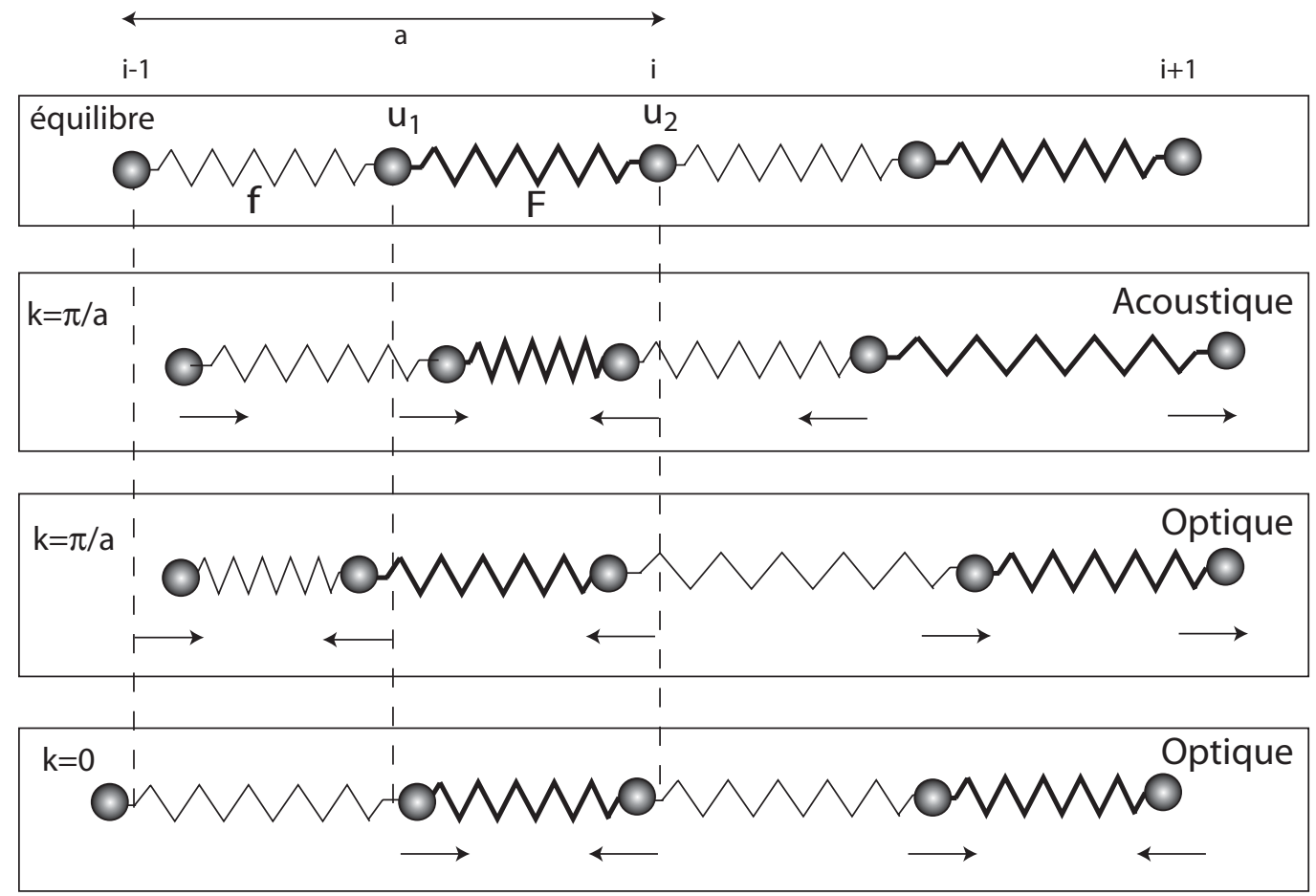

Figure 22. Chaîne linéaire avec motif à deux ressorts alternés. Déplacement pour le mode optique à $k=0$ et pour le mode acoustique (sollicitation uniquement du ressort faible) et optique (sollicitation uniquement du ressort fort) à $k=\pi / a$. Quand la différence entre les deux ressorts est très importante, le ressort fort ne va guère s'allonger dans le cas des modes de la branche acoustique. Inversement, le ressort faible ne va guère s'allonger pour les modes optiques. Le ressort fort crée dans cette limite une entité rigide composée de deux ions, qui n'est pas deformée par les modes acoustiques et qui vibre "en interne" pour les modes optiques. On a affaire à un système moléculaire unidimensionnel.

L'inversion change l'atome 1 en atome 2 ainsi que le vecteur d'onde $\mathrm{k}$ en -k

$$
\begin{aligned}
I: & k \leftrightarrow-k \\
1 & \leftrightarrow 2 .
\end{aligned}
$$

Ceci implique que pour les vecteurs propres

$$
\begin{aligned}
& I\left[e_{ \pm}(k \mid 1)\right]=e_{ \pm}(-k \mid 2), \\
& I\left[e_{ \pm}(k \mid 2)\right]=e_{ \pm}(-k \mid 1) .
\end{aligned}
$$

Comme le système est invariant, nous avons

$$
\begin{aligned}
& I\left[e_{ \pm}(k \mid 1)\right]=e_{ \pm}(k \mid 1), \\
& I\left[e_{ \pm}(k \mid 2)\right]=e_{ \pm}(k \mid 2),
\end{aligned}
$$

ce qui donne les relations suivantes

$$
\begin{aligned}
& e_{ \pm}(k \mid 1)=e_{ \pm}(-k \mid 2), \\
& e_{ \pm}(k \mid 2)=e_{ \pm}(-k \mid 1) .
\end{aligned}
$$


En inspectant les équations de mouvements 3.79 nous constatons immédiatement, que

$$
\left(\begin{array}{c}
e_{ \pm}(k \mid 1)^{*} \\
e_{ \pm}(k \mid 2)^{*}
\end{array}\right)
$$

est une solution et donc un vecteur propre pour le vecteur d'onde $-k$. Ceci est une propriété générale de la matrice dynamique sur laquelle nous reviendrons plus tard. Ce vecteur propre doit donc correspondre au vecteur propre obtenu en appliquant l'opération de symétrie à part une phase commune

$$
\begin{aligned}
& e_{ \pm}(k \mid 1)^{*}=e^{i \phi} e_{ \pm}(k \mid 2), \\
& e_{ \pm}(k \mid 2)^{*}=e^{i \phi} e_{ \pm}(k \mid 1) .
\end{aligned}
$$

En posant

$$
\begin{aligned}
& e_{ \pm}(k \mid 1)=\left|e_{ \pm}(k \mid 1)\right| e^{i \phi_{1}}, \\
& e_{ \pm}(k \mid 2)=\left|e_{ \pm}(k \mid 2)\right| e^{i \phi_{2}},
\end{aligned}
$$

nous obtenons les contraintes

$$
\begin{aligned}
& \left|e_{ \pm}(k \mid 1)\right| e^{-i \phi_{1}}=\left|e_{ \pm}(k \mid 2)\right| e^{i\left(\phi_{2}+\phi\right)}, \\
& \left|e_{ \pm}(k \mid 2)\right| e^{-i \phi_{2}}=\left|e_{ \pm}(k \mid 1)\right| e^{i\left(\phi_{1}+\phi\right)} .
\end{aligned}
$$

Donc

$$
\left|e_{ \pm}(k \mid 1)\right|=\left|e_{ \pm}(k \mid 2)\right|,
$$

c'est-à-dire que les amplitudes pour les deux atomes doivent être identiques pour tous les vecteurs $k$. C'est exactement le résultat qu'on a obtenu par le calcul explicite. Pour les phases, la contrainte

$$
\phi_{1}+\phi_{2}=-\phi
$$

est triviale dû au fait que $\phi$ est une phase arbitraire. Comme nous avons démontré de manière formelle, les mouvements relatifs des deux atomes ne peuvent varier que d'une phase. ${ }^{52}$ La variation de cette phase est tracée dans la figure 23 pour diverses valeurs du rapport $F / f$. A mesure que ce rapport s'éloigne de 1, on assiste au passage d'un cristal normal (dit ionique) à un cristal moléculaire.

Nous allons maintenant analyser les dispersions au centre et au bord de la zone de Brillouin.

\footnotetext{
52 L'apport de la symétrie pour déterminer les phases peut être demontré facilement en centre de zone. La symétrie qui relie les deux atomes du motif, est l'inversion par rapport au centre. Au centre de la zone de Brillouin l'inversion laisse le vecteur $\vec{k}$ inchangé. Par conséquent l'application de l'inversion au vecteur propre doit donner un autre vecteur propre de la même fréquence. Comme nous avons vu, un vecteur propre est défini à une phase arbitraire près. Etant donné que les modes de la chaîne ne sont pas dégénérés, l'image du vecteur propre ne peut différer de l'original que d'une phase.
}

$$
f_{\text {reflection }}(\vec{u})=e^{i \phi} \vec{u}
$$

Faire agir l'inversion une deuxième fois doit nous ramener au point de départ. Donc

$$
f_{\text {reflection }}^{2}(\vec{u})=e^{2 i \phi} \vec{u}=\vec{u} .
$$

Par conséquent

$$
e^{2 i \phi}=1
$$

ou

$$
\phi=0, \pi \text {. }
$$

La réflection correspond à l'échange des deux atomes. Donc

$$
\left(\begin{array}{l}
u(1) \\
u(2)
\end{array}\right)= \pm\left(\begin{array}{l}
u(2) \\
u(1)
\end{array}\right),
$$

c'est-à-dire les modes sont soit symétriques, soit antisymétriques par rapport à l'inversion. En autres mots soit $e( \pm, 0 \mid 1)=$ $e( \pm, 0 \mid 2)$ soit $e( \pm, 0 \mid 1)=-e( \pm, 0 \mid 2)$. Nous avons donc trouvé "l'image" des vecteurs propres en centre de zone de Brillouin sans aucun calcul. 

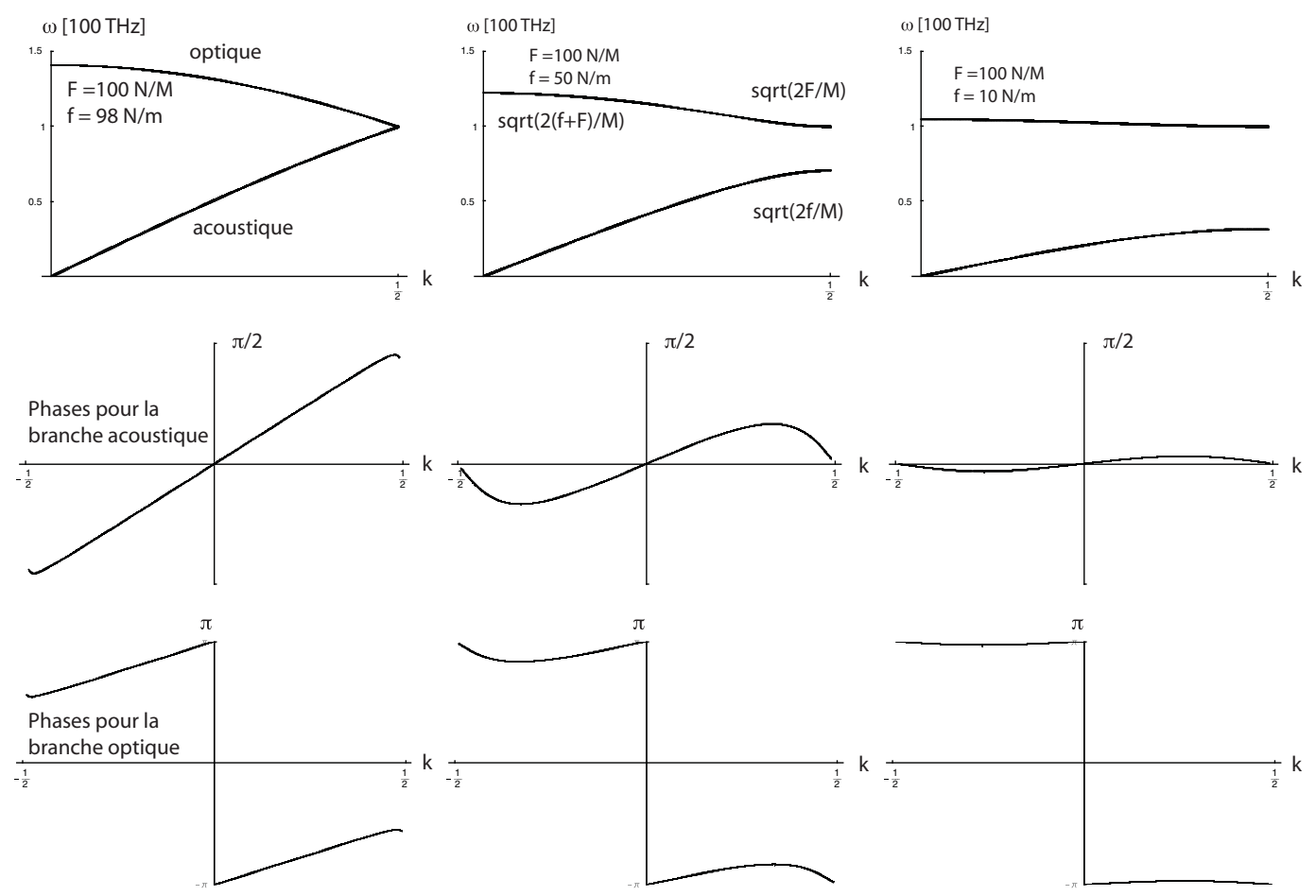

Figure 23. Chaîne linéaire avec un motif à deux atomes créé par deux ressorts $f$ et $F$ en alternance. $k$ est en unité de $\pi$. Pour $F \approx f$ (images de gauche) on obtient une loi de dispersion qui est très similaire à celle de la chaîne monoatomique, si on prend en compte le dédoublement de la maille primitive suite à l'introduction du motif à deux atomes. Le fait que $f$ soit légèrement inférieure à $F$ se reflète seulement dans l'ouverture d'une petite lacune en bord de zone. Cette lacune dans la loi de dispersion entraîne des changements abrupts dans les phases des vecteurs propres. En fait, ces phases doivent être strictement zéro ou $\pi$ en bord de zone selon l'expression 3.82. Dans le cas limite de $F=f$ on a affaire à deux modes dégénérés en bord de zone avec des phases de $\pm \pi / 2$, respectivement. Ces modes peuvent être combinés pour donner des modes avec phase zéro et $\pi$ comme c'est requis par l'expression 3.82. En augmentant le rapport de $F / f$, les deux branches de dispersion se séparent de plus en plus. La branche supérieure devient plate et la phase du mouvement des deux particules reste très proche de $\pi$. C'est le comportement attendu d'une vibration moléculaire peu perturbée par son environnement. Inversement, les particules bougent en phase l'une avec l'autre pour les modes de la branche basse. C'est ce qu'on attend pour les modes acoustiques d'une unité rigide. Les maxima et minima de la courbe de phase indiquent très clairement où, et avec quelle intensité, le caractère des modes diffère de ce que l'on attend d'une chaîne avec $F=f$.

- Centre de zone : La branche à basse fréquence est un mode acoustique standard. Tous les ions bougent en phase et avec des amplitudes identiques $\left(e_{-}(k \mid 1)=e_{-}(k \mid 2)\right.$ pour $\left.k \rightarrow 0\right)$. La dispersion est régie par l'expression linéaire en $k a$

$$
\omega_{-}(k)_{k \rightarrow 0}=\sqrt{\frac{f F}{2 M(f+F)}}|k a|=\sqrt{\frac{\bar{f}}{2 M}}|k a| .
$$

On constate que les inverses des raideurs s'additionnent

$$
\frac{f F}{F+f}=\left(\frac{1}{f}+\frac{1}{F}\right)^{-1}=\bar{f} .
$$

La masse de la chaîne monoatomique $\mathrm{M}$ prend le relais de masse moyenne par maille vue dans le cas précédent. Si un des deux ressorts est beaucoup plus faible que l'autre, il dominera la 
dispersion de la branche acoustique et donc la vitesse du son. Ce sont les ressorts faibles qui donnent la raideur classique d'une chaîne. Transposé en langage chimique, ce sont les forces entre molécules qui déterminent la vitesse du son dans un cristal moléculaire.

La branche optique en centre de zone a la fréquence

$$
\omega_{+}(0)=\sqrt{\frac{2(f+F)}{M}} .
$$

Elle est donc régie par la somme des raideurs des deux ressorts. C'est compréhensible car pour ce mode, une particule se déplace en opposition de phase par rapport à sa voisine, mettant simultanément en jeu les deux ressorts : l'un en traction et l'autre en compression.

- Bord de zone : Nous obtenons pour les deux fréquences

$$
\begin{aligned}
& \omega_{-}\left(\frac{\pi}{a}\right)=\sqrt{\frac{2 f}{M}}, \\
& \omega_{+}\left(\frac{\pi}{a}\right)=\sqrt{\frac{2 F}{M}} .
\end{aligned}
$$

Les vecteurs propres associés sont caractérisés par

$$
\begin{aligned}
& e_{-}\left(\frac{\pi}{a} \mid 1\right)=e_{-}\left(\frac{\pi}{a} \mid 2\right), \\
& e_{+}\left(\frac{\pi}{a} \mid 1\right)=-e_{+}\left(\frac{\pi}{a} \mid 2\right) .
\end{aligned}
$$

Nous sommes en face d'une situation où un ressort sur deux n'est pas sollicité. Il faut remarquer que tous les ions participent au mouvement et que pour cette raison, les déplacements sont très différents de ceux obtenus pour la chaîne diatomique à ressort unique, où un ion sur deux se trouvait au repos en bord de zone. Si la différence entre les deux ressorts est très importante, on aura une grosse lacune entre les deux branches. Cette séparation des échelles d'énergie est un phénomène qui définit un cristal moléculaire. Nous rappellons que dans notre modèle linéaire, la molécule est constituée de paires d'atomes reliés par le ressort fort. La branche optique correspond aux vibrations internes de cette molécule diatomique. D'une maille à l'autre, ces vibrations sont en phase à $k=0$ et en opposition de phase à $k=\pi / 2$. La différence en fréquence des modes, en ces deux endroits de la zone de Brillouin, est imputable au ressort faible, aussi bien dans le cas de la branche acoustique que de la branche optique. La branche acoustique pour laquelle la molécule est une entité rigide de masse $2 M$ est même définie dans son intégralité par ce ressort.

Pour le mode optique, nous obtenons formellement pour la fréquence en centre de zone

$$
\sqrt{\frac{2(f+F)}{M}} \approx \sqrt{\frac{2 F}{M}}\left(1+\frac{1}{2} \frac{f}{F}\right), \quad \text { for } \quad f \ll F,
$$

et

$$
e_{+}(0 \mid 1) \approx-e_{+}(0 \mid 2)
$$

un résultat qui reste valable pour toute la branche optique. Ceci est à comparer à la valeur $\sqrt{2 F / M}$ en bord de zone. Par conséquent, les modes optiques vont produire une bande de fréquence très étroite, de l'ordre de $0.5 f / F$ de sa valeur centrale. Si nous prenons $f / F=0.1$ et une énergie optique typique de $\hbar \sqrt{2 F / M}=30 \mathrm{meV}$ la largeur de la bande optique serait de $1.5 \mathrm{meV}$. La fréquence acoustique en bord de zone se calcule comme $\hbar \sqrt{2 f / M} \approx 10 \mathrm{meV}$. Entre les deux branches, il y a un gap de $20 \mathrm{meV}$. Ce sont des valeurs assez proches de celles que l'on trouvent pour le $\mathrm{C}_{60}$ à l'état solide (voir section 3.16 ainsi que le chapitre de cet ouvrage entièrement consacré à la dynamique des fullerènes). 
a

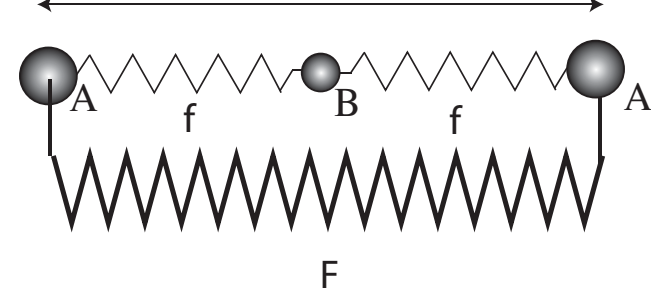

$\mathrm{F}$

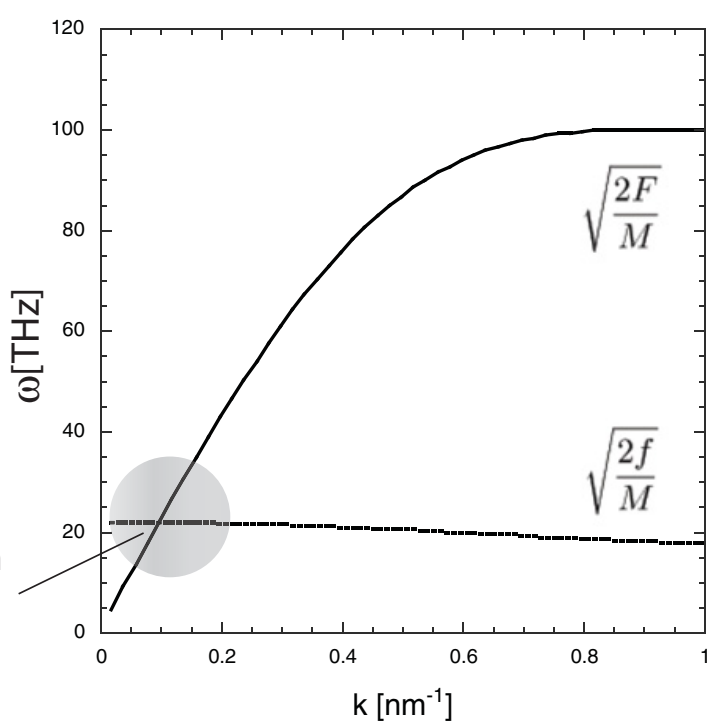

Figure 24. Chaîne linéaire avec un motif à deux atomes de masses différentes. Image qualitative (voir texte). Les ressorts très forts entre les atomes de type $A$ créent des cages pour les atomes de type $B$. La relation de dispersion comporte un mode acoustique dominé dans sa forme par les cages et un mode optique des prisonniers dans les cages.

\subsubsection{Chaîne linéaire : prisonniers en cage}

Nous voudrions terminer notre discussion sur les chaînes linéaires en traitant l'exemple où une particule est mise en cage par ses voisins. Pour cela introduisons dans une chaîne diatomique des ressorts très rigides entre les voisins d'un même type $A$ d'ions (voir figure 24), alors que les ions de type $B$ sont liés de manière faible à leurs voisins de type $A$. Avant d'entamer le calcul exact, tâchons de déterminer le caractère des modes de manière qualitative en utilisant des arguments physiques. Si l'on oublie la présence des ions de type $B$, les relations de dispersion pour les ions de type $A$ ne vont comporter qu'une seule branche acoustique très raide à cause de la forte interaction qui les lie. Concernant les ions de type $B$, ils se voient entourés d'une cage rigide et vibrent comme entre deux murs, avec une fréquence régie par les deux ressorts faibles qui les lient à ces murs. Cela donne une courbe de dispersion très plate à basse fréquence. L'effet est encore plus amplifié si l'ion de type $B$ est plus lourd que son voisin de type $A$. Par nécessité mathématique, les prisonniers de type $B$ suivront leurs cages en phase et en amplitude lors de l'excitation des modes acoustiques de très basse fréquence, c'est-à-dire de très grande longueur d'onde. En contrepartie, les cages doivent compenser le moment cinétique de leurs prisonniers pour les modes optiques. En centre de zone cela implique un mouvement en opposition de phase par rapport à celui des prisonniers. Ce mouvement néanmoins n'allongera pas le ressort fort et, par conséquent, ne changera pas la fréquence du mode optique. En bord de zone, le mouvement des prisonniers dans des cellules voisines est en opposition de phase, ne nécessitant donc aucune compensation de l'impulsion. Les cages peuvent simplement rester au repos. En résumé le mode optique est très plat, et ce n'est que dans le domaine où les deux branches (acoustique et optique) se croisent que des oscillations complexes peuvent se développer, signifiant que les deux sous-systèmes sont fortement couplés. On assiste alors au phènomène "d'anti-croisement" qu'on traitera en détail plus loin dans ce chapitre. Au delà de cette région de la zone de Brillouin, les deux sous-systèmes se découplent rapidement.

Pour vérifier notre raisonnement, calculons explicitement les courbes de dispersion de la chaîne avec les prisonniers en cage. Le ressort faible, qui lie les prisonniers de type $B$ aux cages, est caractérisé par 
sa raideur $f$. Les cages constituées d'atomes de type $A$ sont rigidifiées par l'intermédiaire de ressorts rigides de raideur $F$. Les atomes de type $A$ ont la masse $M_{1}$, ceux du type $B$ la masse $M_{2}$. Dans cette notation la matrice dynamique s'écrit comme ${ }^{53}$

$$
\mathbf{D}=-\left(\begin{array}{cc}
\frac{1}{M_{1}}\left(-2 f-4 F \sin ^{2}\left(\frac{1}{2} k a\right)\right) & \frac{1}{\sqrt{M_{1} M_{2}}}(f(1+\exp (i k a)) \\
\frac{1}{\sqrt{M_{1} M_{2}}}(f(1+\exp (-i k a)) & -\frac{2 f}{M_{2}}
\end{array}\right) .
$$

La seule différence avec la matrice dynamique de la chaîne diatomique simple (expression 3.51) est le terme supplémentaire

$$
4 \frac{F}{M_{1}} \sin ^{2}\left(\frac{1}{2} k a\right)
$$

que nous connaissons bien de la chaîne monoatomique (expression 3.24), et qui décrit à lui seul l'interaction forte entre les atomes formant les cages. En déterminant les valeurs propres de cette matrice nous obtenons les courbes de dispersion

$$
\begin{aligned}
& \omega_{ \pm}^{2}=f \frac{M_{1}+M_{2}}{M_{1} M_{2}}+\frac{2 F}{M_{2}} \sin ^{2}\left(\frac{1}{2} k a\right) \pm \frac{1}{M_{1} M_{2}} \\
& \times \sqrt{M_{1} M_{2}\left(\left(-2 f^{2}+2 f^{2} \cos (k a)-8 f F \sin ^{2}\left(\frac{1}{2} k a\right)\right)+\left(f M_{1}+f M_{2}+2 F M_{1} \sin ^{2}\left(\frac{1}{2} k a\right)\right)^{2}\right.} .
\end{aligned}
$$

Les effets dûs à la différence des masses n'étant pas le centre de notre intérêt nous pouvons simplifier cette expression en choisissant $M_{1}=M_{2}=M$

$$
M \omega_{ \pm}^{2}=2 f+2 F \sin ^{2}\left(\frac{1}{2} k a\right) \pm 2 \sqrt{f^{2} \cos ^{2}\left(\frac{1}{2} k a\right)+F^{2} \sin ^{4}\left(\frac{1}{2} k a\right)} .
$$

Les courbes de dispersion pour divers choix des constantes de forces $f$ et $F$ sont montrées dans la figure 25 .

La situation qui nous intéresse le plus correspond à $f \ll F$. Dans ce cas, le mode acoustique change très vite de caractère. Pour des $k$ toujours relativement petits, il devient plat à un niveau de $\sqrt{2 f / M}$, ce qui correspond à sa valeur en bord de zone. Le mode optique commence très bas en centre de zone $\left(\omega_{+}(0)=\sqrt{4 f / M}\right)$ et possède une dispersion positive proche de celle du mode acoustique. Les deux modes sont séparés d'un gap d'une largeur de

$$
\Delta \omega=(2-\sqrt{2}) \sqrt{\frac{f}{M}}
$$

Nous pouvons considérer le gap comme une mesure de l'hybridation (ou interaction) des deux branches qui se croisent. Cette hybridation devient d'autant plus faible que $f$ diminue. Ce n'est pas vraiment surprenant étant donné que le ressort $f$ couple l' hôte aux cages. Quand nous augmentons le rapport $f / F$, la partie plate passe du mode acoustique au mode optique. Pour $f=2 F$, la loi de dispersion a la particularité de posséder un mode optique sans aucune dispersion. Pour des valeurs de $f$ encore plus élevées, nous récupérons la situation normale décrite dans la section 3.3.3 (où les ressorts les plus forts se trouvent entre voisins directs).

\footnotetext{
53 Souvent il est utile de considérer aussi une faible interaction $g$ entre les atomes prisonniers, par exemple quand ceux-ci sont chargés. Dans ce cas la matrice dynamique devient$$
\mathbf{D}=-\left(\begin{array}{cc}
\frac{1}{M_{1}}\left(-2 f-4 F \sin ^{2}\left(\frac{1}{2} k a\right)\right) & \frac{1}{\sqrt{M_{1} M_{2}}}(f(1+\exp (i k a)) \\
\frac{1}{\sqrt{M_{1} M_{2}}}(f(1+\exp (-i k a)) & \frac{1}{M_{2}}\left(-2 f-4 g \sin ^{2}\left(\frac{1}{2} k a\right)\right)
\end{array}\right)
$$ 

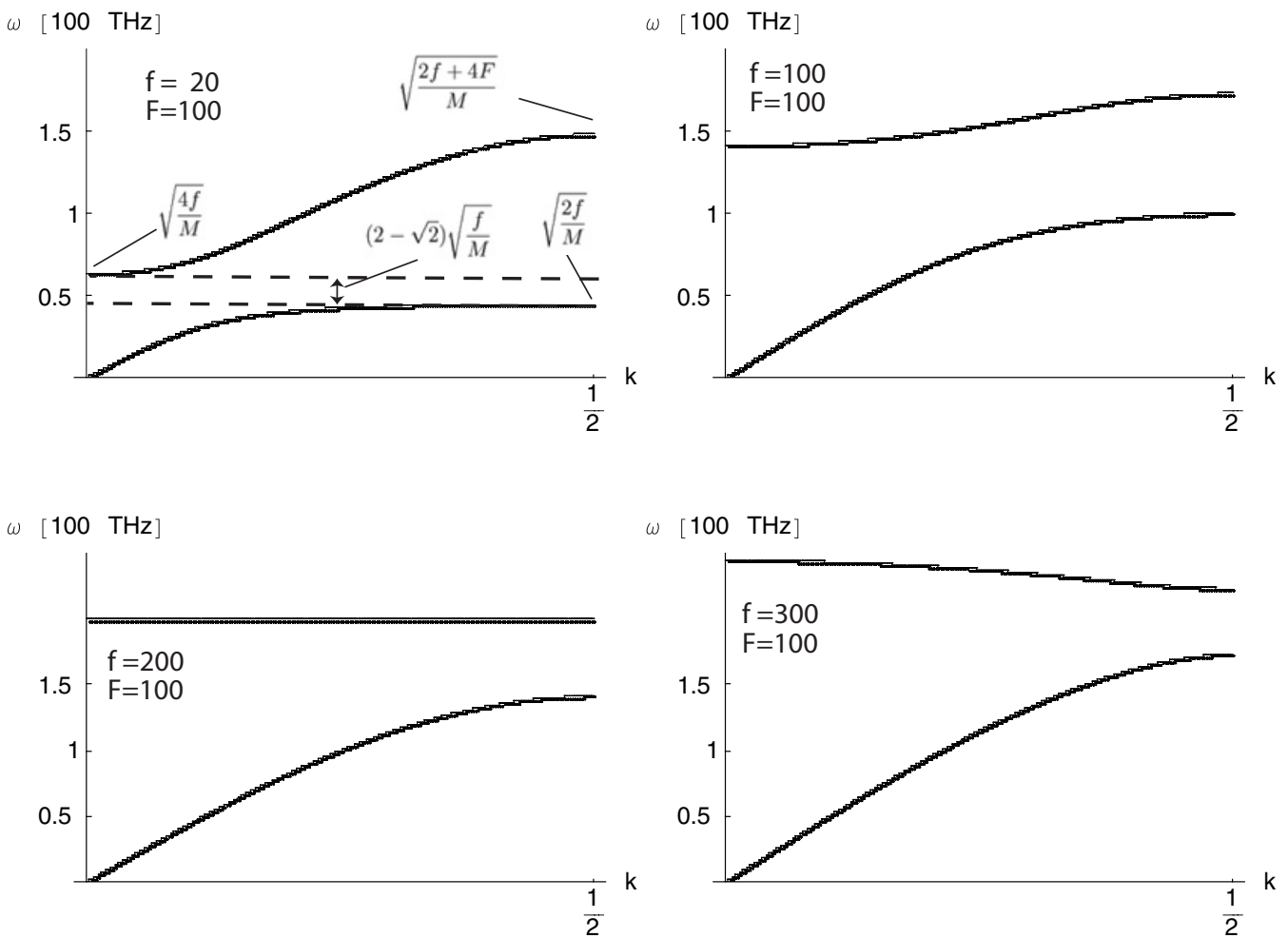

Figure 25. Loi de dispersion pour la chaîne linéaire avec un motif à deux ressorts $f$ et $F$ en unités de N/m. La constante de maille a été choisie de manière à ce que le bord de la zone de Brillouin corresponde à $1 / 2$. Les atomes ont tous la masse 12 u.m.a. Quand le ressort entre les atomes de type $A$ est très fort, il crée des cages pour les atomes de type $B$. La relation de dispersion comporte, dans ce cas, un mode acoustique qui change très vite de caractère et devient plat comme un mode optique. Au fur et à mesure que $f$ augmente (avec $F$ constant), le mode acoustique récupère sa forme habituelle et le mode optique se redresse en centre de zone par rapport au bord. Pour $f=2 F$ on arrive à une singularité dans la densité d'états car la loi de dispersion posséde un mode optique sans aucune dispersion. Pour des valeurs de $f$ encore plus élevées, nous récupérons la situation "normale" que nous avions décrite dans la section 3.3.3.

Le comportement particulier de l'atome prisonnier se voit encore mieux à partir des amplitudes de vibrations. Pour des masses identiques, ces amplitudes doivent coïncider en centre de zone pour le mode acoustique ainsi que pour le mode optique. En s'éloignant du centre de zone, un des deux atomes perd de l'amplitude au profit de l'autre. Pour $f \ll F$, cette perte est très rapide en fonction du vecteur d'onde $k$. À partir du moment où la branche acoustique devient plate, seul l'atome prisonnier oscille. Inversement, il n’y a pratiquement que les cages qui participent aux mouvements de la branche optique. Les deux systèmes d'atomes semblent dynamiquement découplés. Au fur et à mesure que $f$ augmente, ce découplage disparaît au profit d'un comportement normal.

\subsubsection{Chaîne linéaire et oscillateur harmonique}

Nous avons introduit les chaînes linéaires par l'intermédiaire des équations de mouvement, qui relient les accélérations des atomes aux forces exercées par les ressorts mis en action suite aux déplacements mêmes de ces atomes. Pour revenir à notre problème d'origine, c'est-à-dire à la description des excitations dans un système harmonique, il nous faut (i) déduire les équations de mouvement de l'Hamiltonien du système et (ii) établir la connexion entre les constantes de force des ressorts et les 
Amplitudes des déplacements des atomes A formant les cages
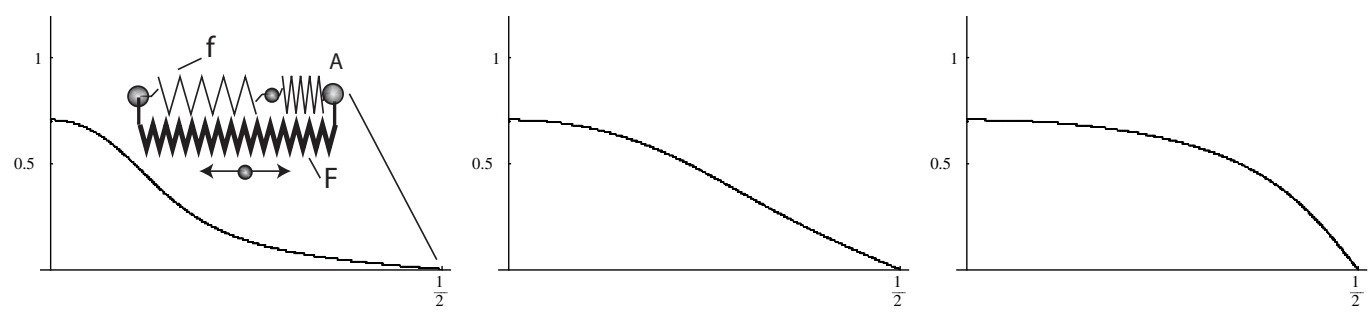

Amplitudes des déplacements des atomes B dans les cages
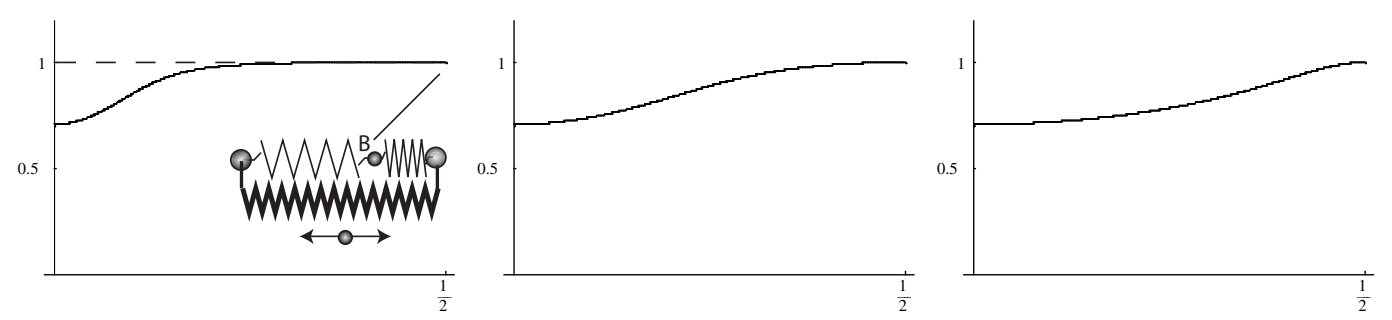

Figure 26. Amplitudes des déplacements liés à la branche inférieure, dont les lois de dispersion sont montrées à la figure 25. Nous ne montrons pas les phases étant donné qu'elles varient de façon linéaire avec le vecteur d'onde $k$. La constante de force $f$ prend les valeurs 20,100 et $300 \mathrm{~N} / \mathrm{m}$. La masse des deux atomes est égale à 12 u.m.a et la raideur du ressort $F$ vaut $100 \mathrm{~N} / \mathrm{m}$. Pour la branche supérieure, la situation est tout simplement inversée (les vecteurs propres pour un vecteur $k$ donné sont orthonormaux). Comme requis pour un mode acoustique, les amplitudes des deux atomes coïncident en centre de zone. Pour le cas $f \ll F$, l'atome $A$ perd rapidement son amplitude dès qu'on s'éloigne du centre de zone. À partir du moment où la branche devient plate (voir figure 25) ce n'est pratiquement que l'atome $B$ qui oscille. Au fur et à mesure que la raideur du ressort $f$ augmente, l'atome $A$ résiste de mieux en mieux à l'arrêt de son mouvement, c'est-à-dire qu'il garde son amplitude d'oscillation jusqu' au voisinage proche du bord de zone.

dérivées d'ordre deux du potentiel par rapport aux déplacements. Dans ce but, transcrivons d'abord l'expression 3.10 pour un système monoatomique linéaire périodique ${ }^{54}$

$$
U_{\text {harm }}\left(x_{1}, \ldots x_{N}\right)=\left.\frac{1}{2} \sum_{n=1}^{N} \sum_{n^{\prime}=1}^{N} \frac{\partial^{2} U}{\partial u(n) \partial u\left(n^{\prime}\right)}\right|_{0} u(n) u\left(n^{\prime}\right),
$$

ce qui nous donne le potentiel dans l'approximation harmonique. Il est à relier au potentiel réel du système. L'énergie élastique d'un système composé de ressorts, dépend des élongations de ces ressorts au carré. Elle s'exprime donc comme ${ }^{55}$

$$
U_{\mathrm{el}}=\frac{1}{2} \sum_{n, n^{\prime}=1}^{N} \frac{1}{2} \Phi\left(n, n^{\prime}\right)\left(u(n)-u\left(n^{\prime}\right)\right)^{2}
$$

\footnotetext{
54 Dans la suite de cette partie, nous omettrons l'indice temporel $t$ afin d'alléger l'écriture. Nous le remettrons dès que la dépendance en temps apparaîtra fondamentale pour la discussion.

55 Le premier facteur $1 / 2$ prend soin du double comptage des paires distinctes d'atomes, comme cela arrive d'habitude dans ce genre de somme. Le deuxième facteur $1 / 2$ assure que la définition de la constante de force est conforme à la loi de Hooke et donc à son utilisation dans nos équations de mouvement.
} 
avec $\Phi\left(n, n^{\prime}\right)$ la constante de force du ressort qui se trouve entre les atomes $n$ et $n^{\prime}$. À partir de ce potentiel nous pouvons calculer les dérivées

$$
\begin{aligned}
\frac{\partial U_{\mathrm{el}}}{\partial u\left(n^{\prime \prime}\right)} & =\frac{1}{2} \sum_{n, n^{\prime}=1}^{N}\left(\Phi\left(n, n^{\prime}\right)\left(u\left(n^{\prime}\right)-u(n)\right) \delta_{n^{\prime}, n^{\prime \prime}}-\Phi\left(n, n^{\prime}\right)\left(u\left(n^{\prime}\right)-u(n)\right) \delta_{n, n^{\prime \prime}}\right) \\
& =-\sum_{n=1}^{N} \Phi\left(n, n^{\prime \prime}\right)\left(u(n)-u\left(n^{\prime \prime}\right)\right),
\end{aligned}
$$

où nous avons exploité le fait qu'un ressort est symétrique par rapport à la directon d'élongation et par conséquent $\Phi\left(n, n^{\prime}\right)=\Phi\left(n^{\prime}, n\right)$.

Traité de manière classique, ce potentiel mène donc aux équations du mouvement

$$
M \frac{d^{2} u(n)}{d t^{2}}=F(n)=-\frac{\partial U_{\mathrm{el}}}{\partial u(n)}=\sum_{n^{\prime}=1}^{N} \Phi_{n^{\prime}, j}\left(u\left(n^{\prime}\right)-u(n)\right) .
$$

et l'on retrouve les équations de la chaîne linéaire 3.13. Pour faire le lien avec l'Hamiltonien en approximation harmonique il nous reste à calculer les dérivées d'ordre deux. Nous obtenons

$$
\frac{\partial^{2} U_{\mathrm{el}}}{\partial u(n) \partial u\left(n^{\prime}\right)}=-\Phi\left(n, n^{\prime}\right), \quad \text { pour } n \neq n^{\prime}
$$

et

$$
\frac{\partial^{2} U_{\mathrm{el}}}{\partial u(n)^{2}}=\sum_{\left(n^{\prime} \neq n\right)=1}^{N} \Phi\left(n, n^{\prime}\right)
$$

ou sous forme plus compacte :

$$
\frac{\partial^{2} U_{\mathrm{el}}}{\partial u(n) \partial u\left(n^{\prime}\right)}=-\Phi_{n, n^{\prime}}+\delta_{n, n^{\prime}} \sum_{n^{\prime \prime}=1}^{N} \Phi_{n, n^{\prime \prime}}
$$

Pour $n \neq n^{\prime}$ le résultat est presque trivial. Le potentiel de notre chaîne ne comportant que des termes d'ordre deux dans les déplacements, les constantes de force correspondent, à part le signe, aux coefficients de développement harmonique. Pour le terme diagonal, les choses sont un peu plus compliquées. Le coefficient harmonique se calcule à partir de la somme de tous les élements nondiagonaux des constantes de forces. Cette propriété exprime le fait que la force exercée sur un atome par tous les autres atomes, quand lui seul est déplacé, est simplement l'opposé de la force exercée sur ce même atome quand celui-ci reste en place et que ce sont tous les autres qui sont déplacés. Déplacer tous les atomes dans leur intégralité n'engendre aucune force de rappel. Cette propriété assure donc à elle seule l'existence d'un mode acoustique de fréquence nulle en centre de zone.

Nous pouvons conclure en soulignant que les fonctions d'onde associées aux excitations quantiques de la chaîne pourront être décrites dans le système de coordonnées normales (éq. 3.32) en utilisant le formalisme classique que nous avons développé dans la section 2.1.5. Pour considérer les détails de cette procédure, revenons au cas général.

\subsection{La matrice dynamique dans l'espace direct}

Reprenons nos réflexions dès le début. Dans l'approximation adiabatique, nous avons découplé le système des ions du système des électrons. En faisant l'approximation harmonique selon 3.10, nous avons réduit le calcul des excitations du réseau dans un potentiel exact au calcul des fonctions d'onde des ions dans un potentiel ne prenant en compte que les déplacements des ions à l'ordre deux. 
Mathématiquement, il nous reste donc à trouver les fonctions propres

$$
\psi_{I}\left(\vec{R}_{1}, \ldots \vec{R}_{N}\right)
$$

de l'Hamiltonien

$$
\mathbf{H}=\sum_{I} \frac{\hbar^{2}}{2 M_{I}} \vec{\nabla}_{I}^{2}+U\left(\vec{R}_{1}, \ldots \vec{R}_{N}\right)
$$

avec le potentiel harmonique donné par éq. 3.10

$$
U\left(\vec{R}_{1}, \ldots \vec{R}_{N}\right)=U_{0}+\left.\sum_{\alpha, n=1}^{3, N} \frac{\partial U}{\partial u_{\alpha}(n)}\right|_{0} u_{\alpha}(n)+\left.\frac{1}{2} \sum_{\alpha, n=1}^{3, N} \sum_{\beta, n^{\prime}=1}^{3, N} \frac{\partial^{2} U}{\partial u_{\alpha}(n) \partial u_{\beta}\left(n^{\prime}\right)}\right|_{0} u_{\alpha}(n) u_{\beta}\left(n^{\prime}\right) .
$$

Comme dans le cas des oscillateurs couplés, que nous avons traité dans la section 2.1.5, nous devons changer les coordonnées de manière à aboutir à une séparation des variables. Ne pas être obligé de prendre en compte le caractère d'opérateur des variables est un avantage considérable lors de l'exécution des calculs. Pour cette raison, nous établirons cette séparation en passant par les équations de mouvement classiques. La séparation est acquise quand nous avons identifié les modes normaux avec leurs fréquences propres. Une fois la séparation effectuée, nous passerons à la mécanique quantique en associant à chaque mode (ou coordonnée) normal, ainsi qu'à son moment cinétique associé, un opérateur quantique. Ecrit avec l'aide de ces opérateurs, l'Hamiltonien deviendra une somme d'oscillateurs harmoniques découplés dont nous pouvons trouver les fonctions propres.

Dans ce contexte, il est utile de se rappeler que les équations classiques de mouvement valent aussi pour les valeurs moyennes les plus probables des positions et des moments cinétiques. Formellement on peut exprimer cette assertion comme

$$
\begin{aligned}
& \frac{d}{d t}\left\langle\mathbf{Q}_{i}\right\rangle=\left\langle\frac{\partial \mathbf{H}}{\partial \mathbf{P}_{i}}\right\rangle=\frac{\partial \mathbf{H}\left(\left\{\left\langle\mathbf{Q}_{j}\right\rangle,\left\langle\mathbf{P}_{j}\right\rangle\right\}\right)}{\partial\left\langle\mathbf{P}_{i}\right\rangle}, \\
& \frac{d}{d t}\left\langle\mathbf{P}_{i}\right\rangle=-\left\langle\frac{\partial \mathbf{H}}{\partial \mathbf{Q}_{i}}\right\rangle=-\frac{\partial \mathbf{H}\left(\left\{\left\langle\mathbf{Q}_{j}\right\rangle,\left\langle\mathbf{P}_{j}\right\rangle\right\}\right)}{\partial\left\langle\mathbf{Q}_{i}\right\rangle} .
\end{aligned}
$$

C'est une manifestation du théorème d'Ehrenfest [4], qui stipule que les valeurs moyennes des positions et des impulsions satisfont aux mêmes équations du mouvement que les variables classiques, si $\mathbf{Q}_{i}$ et $\mathbf{P}_{i}$ n'apparaissent qu'à l'ordre 1 et 2 dans l'Hamiltonien. $Q_{i}$ et $P_{i}$ désignent ici les coordonnées canoniques. ${ }^{56}$ Les trajectoires suivies par les centres des paquets d'ondes, qu'on pourra former dans un système harmonique, obéiront donc rigoureusement aux lois de la mécanique classique. Mais attention, les observables liées à une fonction propre de l'Hamiltonien sont stationnaires, c'est-à-dire qu'elles ne changent pas avec le temps. Pour cette raison, un état quantique propre ne décrit jamais une oscillation classique. Cette assertion est vraie en dépit du fait que l'énergie et la longueur d'onde d'un état quantique correspondent toujours à la fréquence et à la longueur d'onde d'une onde classique du système. On doit donc toujours rester prudent quand on invoque l'analogie avec le système classique. Nous allons revenir sur ce point quand nous parlerons des états cohérents.

Pour franchir le premier pas vers une diagonalisation de l'Hamiltonien, nous sommes donc confrontés au problème de trouver les modes normaux et les fréquences propres $\omega_{j}$ des équations de

\footnotetext{
56 En dynamique des réseaux, on préfère en général travailler avec les déplacements $\mathbf{u}(\vec{R})$ au lieu de $\mathbf{r}(\vec{R})$ comme coordonnées canoniques; autrement dit, chaque atome est repéré par rapport à une origine différente qui est sa position à l'équilibre.
} 


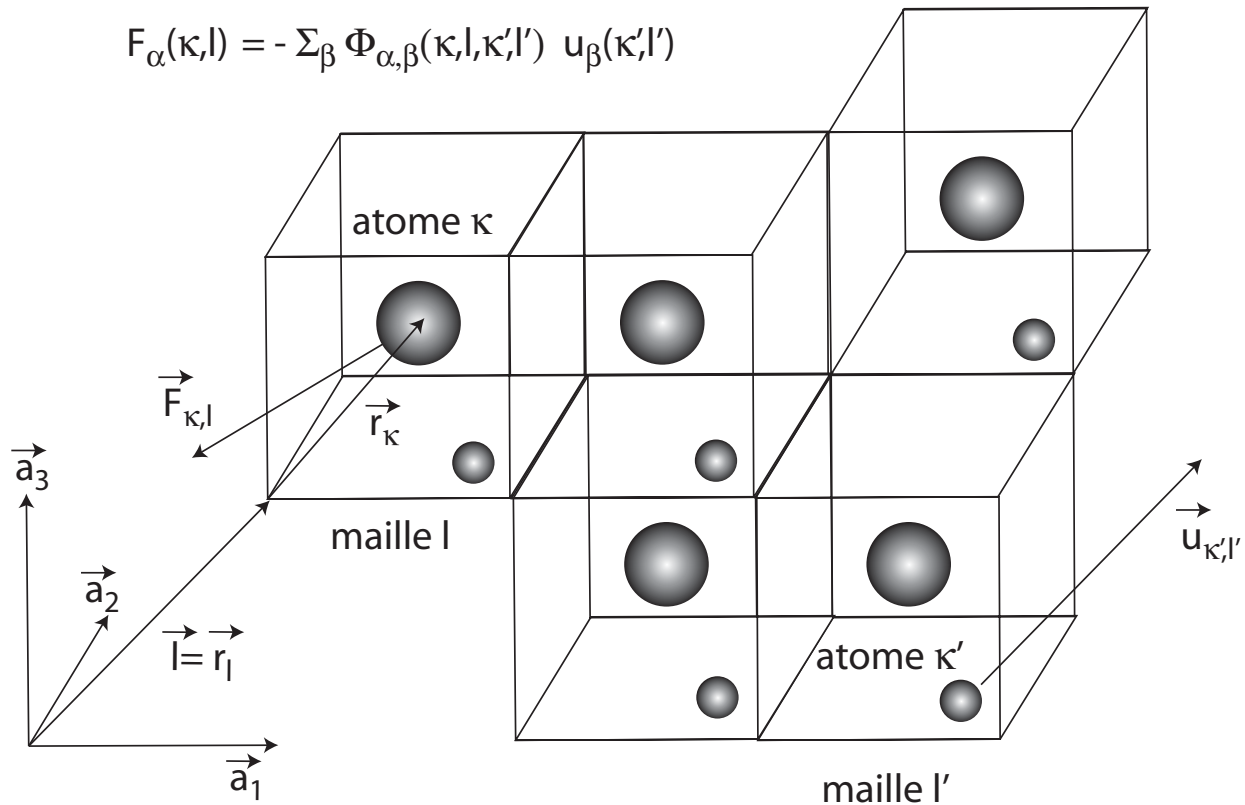

Figure 27. Constantes de force schématiques pour un cristal de deux atomes $(\kappa=1,2)$ dans la maille primitive. Lors du déplacement $\vec{u}\left(\kappa^{\prime}, \vec{l}^{\prime}\right)$ de l'atome $\kappa^{\prime}$ de la maille $l^{\prime}$, une force agit sur l'atome $\kappa$ de la maille $l$. Cette force peut se calculer à partir des constantes de force selon la formule indiquée dans la figure.

mouvement classiques. Ces équations décrivent un système mécanique tridimensionnel de $N$ particules de masses $m_{n}, n=1, \ldots N$ connectées par des forces d'interaction classiques.

Il s'avère utile de reformuler le problème en introduisant des matrices. Les dérivées secondes

$$
\Phi_{\alpha \beta}\left(n, n^{\prime}\right)=-\left.\frac{\partial^{2} U}{\partial u_{\alpha}(n) \partial u_{\beta}\left(n^{\prime}\right)}\right|_{0}
$$

sont les constantes de force du matériau. Elles déterminent la force de rappel que subit l'ion $n$ suivant la direction $\vec{a}_{\alpha}$, quand l'ion $n^{\prime}$ se déplace suivant $\vec{a}_{\beta}$ (voir figure 27). Nous en avions rencontrés des exemples concrets lors de la discussion de la chaîne linéaire. Les constantes de force peuvent être considérées comme les éléments d'une matrice $\tilde{\Phi}$ de dimension $(3 N \times 3 N){ }^{57}$

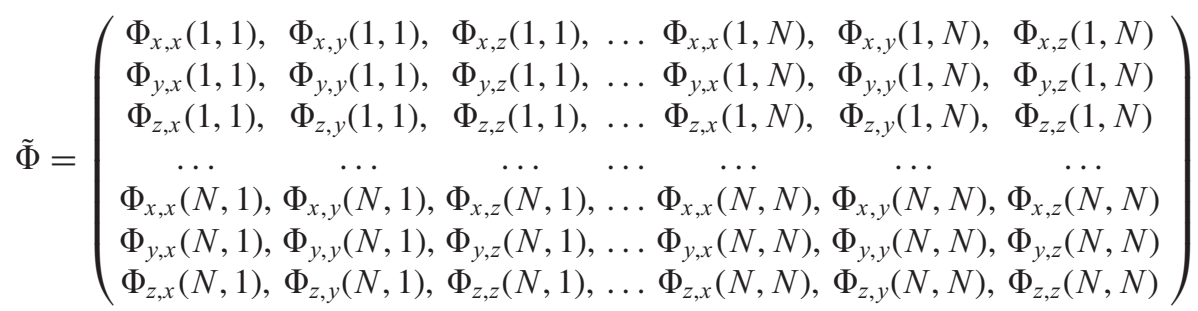

Une attention toute particulière doit être accordée aux termes à un atome (ou termes "self") $\Phi_{\alpha \beta}(n, n)$. Souvent on ne peut les déterminer directement, mais on les évalue en utilisant le principe

\footnotetext{
57 Nous noterons les matrices ici avec une tilde.
} 
d'action-réaction

$$
\Phi_{\alpha \beta}(n, n)=-\sum_{n^{\prime} \neq n} \Phi_{\alpha \beta}\left(n, n^{\prime}\right)
$$

On trouvera des détails dans la référence [20]. Nous définissons la matrice dite dynamique ${ }^{58}$ à partir de la matrice des constantes de force par

$$
D_{\alpha \beta}\left(n, n^{\prime}\right)=\left.\frac{1}{\sqrt{m_{n} \cdot m_{n^{\prime}}}} \frac{\partial^{2} U}{\partial u_{\alpha}(n) \partial u_{\beta}\left(n^{\prime}\right)}\right|_{0}=-\frac{\Phi_{\alpha \beta}\left(n, n^{\prime}\right)}{\sqrt{m_{n} \cdot m_{n^{\prime}}}}
$$

où $m_{n}$ désigne la masse de l'ion $\mathrm{n}$.

A l'aide des notations matricielles

$$
\tilde{D}=-\tilde{M}^{-\frac{1}{2}} \tilde{F} \tilde{M}^{-\frac{1}{2}} \quad \text { avec } \quad \tilde{M}_{\alpha, \beta}^{-\frac{1}{2}}\left(n, n^{\prime}\right)=\frac{1}{\sqrt{m_{n}}} \delta_{\alpha, \beta} \delta_{n, n^{\prime}}
$$

Nous introduisons le vecteur à $3 N$ composantes pondéré par les racines carrées de la masse des ions

$$
\overrightarrow{\bar{u}}_{3 N}=\left(\sqrt{m_{1}} \vec{u}(1), \sqrt{m_{2}} \vec{u}(2), \ldots, \sqrt{m_{N}} \vec{u}(N)\right) .
$$

Dans cette base, les équations du mouvement classique

$$
m_{i} \ddot{u}_{\alpha}(n \mid t)=\sum_{\beta, n^{\prime}=1}^{3, N} \Phi_{\alpha \beta}\left(n, n^{\prime}\right) u_{\beta}\left(n^{\prime} \mid t\right)
$$

s'écrivent comme

$$
\ddot{\bar{u}}_{\alpha}(n \mid t)=-\sum_{n^{\prime}=1, \beta=1}^{N, 3} D_{\alpha \beta}\left(n, n^{\prime}\right) \bar{u}_{\beta}\left(n^{\prime} \mid t\right) .
$$

Nous cherchons des solutions oscillatoires pour lesquelles

$$
\bar{u}_{\alpha}(n \mid t) \propto \cos (\omega t+\phi), \quad \forall n=1, \ldots N, \quad \forall \alpha=1,2,3,
$$

c'est-à-dire caractérisées par des fréquences $\omega$ et des phases $\phi$.

Dans ce cas les équations du mouvement deviennent

$$
\omega^{2} \overrightarrow{\bar{u}}_{3 N}=\tilde{D} \overrightarrow{\bar{u}}_{3 N}
$$

$\tilde{D}$ étant une matrice réelle et symétrique

$$
\tilde{D}=\tilde{D}^{T}
$$

elle peut être diagonalisée. Ses $3 N$ valeurs propres $\omega_{j}^{2}$ sont réelles. Si elles sont en plus positives nous obtenons des fréquences $\omega_{j}$ positives. Des valeurs propres $\omega_{j}^{2}$ négatives conduisent à des fréquences $\omega_{j}$ imaginaires, et donc non pas à des oscillations mais à des mouvements de relaxation. Ces relaxations indiquent que la structure de départ n'est pas stabilisée par les forces harmoniques. Les $3 N$ vecteurs propres $\vec{e}_{j}$ forment une base complète réelle, qui peut être choisie orthonormale [20]

$$
\begin{gathered}
\vec{e}_{j} \cdot \vec{e}_{j^{\prime}}=\sum_{\alpha, n=1}^{3, N} e_{j}(\alpha, n) e_{j^{\prime}}(\alpha, n)=\delta_{j, j^{\prime}}, \\
\sum_{j=1}^{3 N} e_{j}(\alpha, n) e_{j}\left(\beta, n^{\prime}\right)=\delta_{\alpha, \beta} \delta_{n, n^{\prime}} .
\end{gathered}
$$

\footnotetext{
58 Il est utile de souligner qu'il s'agit ici de la matrice dynamique dans l'espace direct. La matrice dynamique introduite avec les chaînes linéaires était définie dans l'espace des vecteurs d'onde $\vec{k}$.
} 
Par conséquent, tous les mouvements des atomes qui correspondent à une solution des équations de mouvement peuvent s'exprimer comme une combinaison linéaire

$$
\bar{u}_{\alpha}(n \mid t)=\sum_{j=1}^{3 N} Q_{j} e_{j}(\alpha, n) \cos \left(\omega_{j} t+\phi_{j}\right), \quad n=1, \ldots N, \quad \alpha=1,2,3
$$

avec $Q_{j}$ et $\phi_{j}$ les coefficients du développement. Ces coefficients sont déterminés par les conditions initiales. Il ne s'agit donc, dans le fond, que d'un changement de variables d'un espace orthogonal vers un autre plus adapté au traitement des vibrations. Pour chaque mode propre de vibration de fréquence $\omega_{j}$ donnée, l'amplitude du mouvement d'un atome est définie par le vecteur propre correspondant. De plus, tous les atomes partagent la même phase. Dans la terminologie utilisée lors de la discussion des chaînes linéaires, nous dirons que les solutions trouvées sont stationnaires.

Le problème classique est donc résolu si nous connaissons les valeurs et les vecteurs propres de la matrice dynamique, ou si nous savons diagonaliser la matrice dynamique. L'existence des solutions est assurée par le fait que celle-ci est réelle et symétrique. Dans la pratique, il faut trouver les racines du polynôme caractéristique de la matrice donné par

$$
P\left(\omega^{2}\right)=\left|\left(\tilde{D}-\omega^{2} \tilde{I}_{3 N}\right)\right|
$$

où $\tilde{I}_{3 N}$ est la matrice unité de dimension $(3 N \times 3 N)$.

Nous voudrions souligner que jusqu'ici, nous n'avons pas introduit de réseau cristallin. Les vibrations trouvées sont les vibrations propres du système dans l'hypothèse où l'approximation harmonique est valable. Le formalisme s'applique donc aux cristaux aussi bien qu'aux amorphes. En particulier les solutions trouvées ne sont pas encore des ondes. Si on veut faire le rapprochement avec les structures périodiques, on peut toujours considérer le système amorphe comme le motif d'un cristal et se limiter à ne regarder que les modes en centre de zone.

La différence devient évidente si on compare avec l'exemple concret de la matrice dynamique, que nous avons rencontré lors de la discussion de la chaîne linéaire avec un motif à deux atomes. Le grand changement par rapport à la matrice dynamique introduite ici en toute généralité dans l'espace direct, est sa dépendance vis à vis du vecteur d'onde $\vec{k}$. C'est une conséquence de la symétrie de translation, sur laquelle nous allons revenir plus tard dans la section traitant des phonons.

\subsection{Excitations vibrationnelles}

Pour effectuer le passage à la mécanique quantique, nous écrivons l'Hamiltonien 3.122 sous sa forme matricielle

$$
\mathbf{H}=\frac{1}{2} \overrightarrow{\mathbf{p}}_{3 N} \cdot \overrightarrow{\mathbf{p}}_{3 N}+\overrightarrow{\overline{\mathbf{u}}}_{3 N} \tilde{D} \overrightarrow{\overline{\mathbf{u}}}_{3 N}
$$

avec les opérateurs vectoriels de dimension $3 N$ définis par

$$
\begin{aligned}
& \overrightarrow{\overline{\mathbf{u}}}_{3 N}=\left(\sqrt{m_{1}} \overrightarrow{\mathbf{u}}(1), \sqrt{m_{2}} \overrightarrow{\mathbf{u}}(2), \ldots, \sqrt{m_{N}} \overrightarrow{\mathbf{u}}(N)\right), \\
& \overrightarrow{\overline{\mathbf{p}}}_{3 N}=\left(\left(1 / \sqrt{m_{1}}\right) \overrightarrow{\mathbf{p}}(1),\left(1 / \sqrt{m_{2}}\right) \overrightarrow{\mathbf{p}}(2), \ldots,\left(1 / \sqrt{m_{N}}\right) \overrightarrow{\mathbf{p}}(N)\right) .
\end{aligned}
$$

Si nous effectuons un changement de variable vers l'espace des vecteurs propres de la matrice $\tilde{D}$ nous obtenons

$$
\mathbf{H}=\frac{1}{2}\left(\sum_{j=1}^{3 N} \mathbf{P}_{j} \cdot \mathbf{P}_{j}+\omega_{j}^{2}\left(\mathbf{Q}_{j} \cdot \mathbf{Q}_{j}\right)\right)
$$


où $\mathbf{Q}_{\mathbf{j}}$ et $\mathbf{P}_{\mathbf{j}}$ désignent les opérateurs vectoriels de dimension $3 N$ de la position et de l'impulsion transformés ${ }^{59}$ dans l'espace des modes propres. Formellement, ce changement de variable s'écrit comme

$$
Q_{j}=\sum_{\alpha, n}^{3, N} e_{j}(\alpha, n) \bar{u}_{\alpha}(n)
$$

ou en notation matricielle

$$
\begin{aligned}
\overrightarrow{\mathbf{Q}}_{3 N} & =\tilde{S} \overrightarrow{\overline{\mathbf{u}}}_{3 N} \\
\overrightarrow{\mathbf{P}}_{3 N} & =\tilde{S} \overrightarrow{\overline{\mathbf{p}}}_{3 N}
\end{aligned}
$$

avec

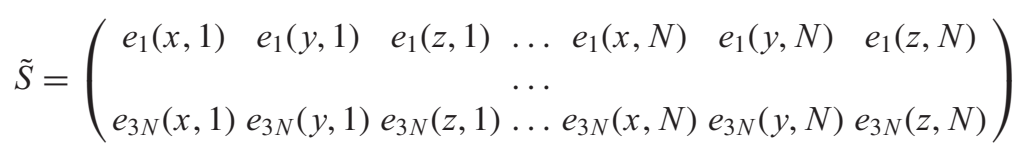

définie comme la matrice orthogonale de changement de variable de dimension $3 N \times 3 N$, dont les lignes sont formées par les vecteurs propres. C'est à ce stade qu'intervient la diagonalisation de la matrice dynamique, que nous avons effectuée au préalable de manière classique. Nous voudrions rappeler que ceci n'a été possible qu'en travaillant dans l'approximation harmonique. Mathématiquement parlant, dans cette approximation l'Hamiltonien s'écrit comme une forme bilinéaire des variables, ce qui nous permet d'appliquer tous les outils de l'algèbre linéaire et en particulier la diagonalisation en base orthonormale.

Nous constatons que les variables sont séparées et nous pouvons donc appliquer le formalisme développé en sections 2.1.2 et 2.1.5 pour trouver les fonctions d'onde. Nous introduisons alors les opérateurs de création $\mathbf{a}_{j}^{+}$et d'annihilation $\mathbf{a}_{j}$ pour chaque mode propre $j$ :

$$
\begin{aligned}
\mathbf{a}_{j}^{+} & =\frac{1}{\sqrt{2}}\left(\hat{\xi}_{j}-i \hat{\pi}_{j}\right), j=1, \ldots 3 N, \\
\mathbf{a}_{j} & =\frac{1}{\sqrt{2}}\left(\hat{\xi}_{j}+i \hat{\pi}_{j}\right), j=1, \ldots 3 N
\end{aligned}
$$

avec

$$
\begin{aligned}
& \hat{\xi}_{j}=\alpha_{j} \mathbf{Q}_{j}, \\
& \hat{\pi}_{j}=\frac{1}{\alpha_{j} \hbar} \mathbf{P}_{j},
\end{aligned}
$$

et

$$
\alpha_{j}=\sqrt{\frac{\omega_{j}}{\hbar}} .
$$

A ce stade, on voit clairement la nécessité de travailler avec les déplacements pondérés par les racines des masses.

Avec ces opérateurs, l'Hamiltonien se simplifie

$$
\mathbf{H}=\sum_{j=1}^{3 N} \hbar \omega_{j}\left(\mathbf{a}_{j}^{+} \mathbf{a}_{j}+\frac{1}{2}\right) .
$$

\footnotetext{
${ }^{59}$ Les composantes de $\mathbf{Q}_{\mathbf{j}}$ sont donc des combinaisons linéaires des opérateurs de position $\overrightarrow{\mathbf{u}}_{3 N}$ et les composantes de $\mathbf{P}_{\mathbf{j}}$ sont des combinaisons linéaires des opérateurs d'impulsion, $\overrightarrow{\mathbf{p}}_{3 N}$, respectivement.
} 
Il se résume à une simple somme d'oscillateurs découplés. Les fonctions d'onde des états excités se déterminent donc à partir de la fonction de l'état fondamental en appliquant les $3 N$ opérateurs de création

$$
\left|\psi_{n_{1}, \ldots, n_{3 N}}\right\rangle \equiv\left|n_{1}, \ldots, n_{3 N}\right\rangle=\prod_{j=1}^{3 N} \frac{\left(\mathbf{a}_{j}^{+}\right)^{n_{j}}}{\sqrt{n_{j} !}}|0, \ldots, 0\rangle .
$$

La fonction $\left|n_{1}, \ldots, n_{3 N}\right\rangle$ spécifie, pour chacun des $3 N$ modes propres de vibration, le niveau $n_{j}$ dans lequel il se trouve. L'énergie d'un état stationnaire -donc propre- de l'Hamiltonien se calcule selon

$$
E=\left\langle n_{1}, \ldots n_{3 N}|\mathbf{H}| n_{1}, \ldots n_{3 N}\right\rangle=\sum_{j=1}^{3 N}\left(n_{j}+\frac{1}{2}\right) \hbar \omega .
$$

Connaissant les vecteurs propres et donc la relation entre les opérateurs $\mathbf{u}$ et $\mathbf{Q}$, nous pouvons inverser les expressions 3.148 en nous appuyant sur le fait que la matrice orthogonale $\tilde{S}$ possède une matrice inverse égale à sa transposée (voir aussi la section 2.1.5)

$$
\tilde{S}^{-1}=\tilde{S}^{t}
$$

L'opérateur de déplacement $\mathbf{u}_{\alpha}(n)$ de l'ion $n$ suivant la direction $\alpha$ est relié aux composantes des vecteurs propres $\vec{e}_{j}$ via la relation ${ }^{60}$

$$
\mathbf{u}_{\alpha}(n)=\sum_{j} \sqrt{\frac{\hbar}{2 m_{n} \omega_{j}}}\left[e_{j}(\alpha, n) \mathbf{a}_{j}+e_{j}^{*}(\alpha, n) \mathbf{a}_{j}^{+}\right] .
$$

Comme on peut montrer facilement en utilisant les expressions 3.148 et 3.149 cette relation correspond à

$$
\sqrt{m_{n}} \mathbf{u}_{\alpha}(n)=\sum_{j} e_{j}(\alpha, n) \mathbf{Q}_{j}
$$

et est donc le pendant de l'équation 3.138 pour les opérateurs. De manière analogue on obtient pour les opérateurs d'impulsion :

$$
\mathbf{p}_{\alpha}(n)=-i \sum_{j} \sqrt{\frac{\hbar \omega_{j} m_{n}}{2}}\left[e_{j}(\alpha, n) \mathbf{a}_{j}^{+}-e_{j}^{*}(\alpha, n) \mathbf{a}_{j}\right] .
$$

Comme les excitations de vibration satisfont à la statistique de Bose-Einstein (voir la section 2.3.3), l'occupation d'un mode $j$ à la température $T$ est donnée par

$$
\left\langle\mathbf{a}_{j}^{+} \mathbf{a}_{j}\right\rangle=n\left(\omega_{j}\right)=\frac{1}{e^{\frac{\hbar \omega_{j}}{k_{B} T}}-1} .
$$

\subsection{Déplacements quadratiques moyens}

Pour avoir des informations sur les déplacements des particules, nous devons regarder les fluctuations liées aux opérateurs $\mathbf{u}_{l}$. Celles-ci peuvent être obtenues avec l'aide des expressions 3.155 et 3.158 en respectant les règles de commutation des opérateurs d'échelle. L'amplitude quadratique moyenne de la

\footnotetext{
${ }^{60}$ Pour garder les relations aussi générales que possible, nous les formulons pour des vecteurs propres complexes, même si dans le cas d'une matrice dynamique dans l'espace direct nous savons que ceux-ci sont réels.
} 
particule $l$ suivant la direction $\alpha$, se calcule comme suit ${ }^{61}$

$$
\begin{aligned}
\overline{u_{\alpha}(n)^{2}} & =\left\langle\mathbf{u}_{\alpha}(n) \mathbf{u}_{\alpha}^{+}(n)\right\rangle \\
& =\sum_{j=1}^{3 N} \frac{\left|e_{j}(\alpha, n)\right|^{2} \hbar}{2 m_{n} \omega_{j}}\left\langle\mathbf{a}_{j} \mathbf{a}_{j}^{+}+\mathbf{a}_{j}^{+} \mathbf{a}_{j}\right\rangle \\
& =\sum_{j=1}^{3 N} \frac{\left|e_{j}(\alpha, n)\right|^{2} \hbar}{2 m_{n} \omega_{j}}\left(1+2\left\langle\mathbf{a}_{j}^{+} \mathbf{a}_{j}\right\rangle\right) \\
& =\sum_{j=1}^{3 N} \frac{\left|e_{j}(\alpha, n)\right|^{2} \hbar}{2 m_{n} \omega_{j}}\left(2 n\left(\omega_{j}\right)+1\right) .
\end{aligned}
$$

Si nous nous limitons à la contribution d'un seul mode en posant

$$
\left|u_{\alpha}(j \mid n)\right|^{2}=\frac{\left|e_{j}(\alpha, n)\right|^{2} \hbar}{2 m_{n} \omega_{j}}\left(2 n\left(\omega_{j}\right)+1\right)
$$

alors on peut écrire 3.159 sous la forme d'une somme de contribution de modes

$$
\overline{u_{\alpha}(n)^{2}}=\sum_{j=1}^{3 N}\left|u_{\alpha}(j \mid n)\right|^{2} .
$$

L'amplitude des déplacements en fonction de la position suit le vecteur propre pour ce mode. Si ce vecteur propre comporte des zéros pour une partie des particules, celles-ci se trouvent classiquement à l'arrêt. Ce n'est pas le cas pour le système quantique, le repos n'étant pas compatible avec la relation d'incertitude de Heisenberg. Etant donné que la fonction d'onde d'une seule excitation est

$$
\left|0, \ldots, n_{j}, \ldots, 0\right\rangle,
$$

ces particules se trouvent simplement dans la superposition des états fondamentaux de tous les autres modes avec leurs incertitudes, qu'on appelle fluctuations quantiques.

Si tous les atomes participent à un mode $j$, alors $\left|e_{j}(\alpha, n)\right|$ est de l'ordre de $\frac{1}{3 N}$, ce qui est très petit à l'échelle globale du système. La situation change quand les modes sont localisés. Une bonne mesure de la participation des atomes à un mode donné $j$ est le rapport de participation [21]

$$
\eta_{j}=\frac{\left(\sum_{\alpha, n=1}^{3, N}\left|u_{\alpha}(j \mid n)\right|^{2}\right)^{2}}{N \sum_{\alpha, n=1}^{3, N}\left|u_{\alpha}(j \mid n)\right|^{4}} .
$$

Pour un déplacement rigide de l'ensemble du système dans la direction $\alpha$ on a

$$
u_{\alpha}(j \mid n) \propto \frac{1}{N}=\text { constante }
$$

61 On se rappelle que

$$
\left\langle\mathbf{a}_{j} \mathbf{a}_{j^{\prime}}\right\rangle=\left\langle\mathbf{a}_{j}^{+} \mathbf{a}_{j^{\prime}}^{+}\right\rangle=0
$$

quelles que soient les valeurs de $j$ et $j^{\prime}$ alors que

$$
\left\langle\mathbf{a}_{j} \mathbf{a}_{j^{\prime}}^{+}\right\rangle=\left\langle\mathbf{a}_{j}^{+} \mathbf{a}_{j^{\prime}}\right\rangle=0
$$

pour $j \neq j^{\prime}$. 
pour tous les atomes $n$, et par la suite

$$
\eta_{j}=\left(\sum_{n}^{N} 1\right)^{2} / N\left(\sum_{n}^{N} 1\right)=1
$$

Pour des modes étendus, $\eta_{j}$ reste proche de 1 , tandis que pour des modes très localisés

$$
\eta_{j} \approx N_{\text {part }} / N
$$

où $N_{\text {part }}$ correspond au nombre d' atomes "participants"62 au mode $j$.

En moyennant l'équation 3.159 sur toutes les directions, nous obtenons

$$
\overline{u^{2}(n)}=\frac{1}{3}\left\langle\overrightarrow{\mathbf{u}}(n) \overrightarrow{\mathbf{u}}^{+}(n)\right\rangle=\frac{1}{N} \sum_{\alpha=1}^{3} \sum_{j=1}^{3 N} \frac{\left|e_{j}(\alpha, n)\right|^{2} \hbar}{2 m_{n} \omega_{j}}\left(2 n\left(\omega_{j}\right)+1\right)
$$

et finalement, en moyennant l'équation 3.164 sur tous les atomes, on obtient le déplacement carré moyen

$$
\overline{u^{2}}=\frac{1}{3 N} \sum_{n=1}^{N}\left\langle\overrightarrow{\mathbf{u}}(n) \overrightarrow{\mathbf{u}}^{+}(n)\right\rangle=\frac{1}{3 N} \sum_{\alpha=1}^{3} \sum_{n=1}^{N} \sum_{j=1}^{3 N} \frac{\left|e_{j}(\alpha, n)\right|^{2} \hbar}{2 m_{n} \omega_{j}}\left(2 n\left(\omega_{j}\right)+1\right) .
$$

Si tous les noyaux ont la même masse, on peut à nouveau simplifier l'expression ci-dessus à l'aide de la relation d'orthogonalité 3.136

$$
\overline{u^{2}}=\frac{1}{3 N} \sum_{j=1}^{3 N} \frac{\hbar}{2 m \omega_{j}}\left(2 n\left(\omega_{j}\right)+1\right) .
$$

Le déplacement carré moyen est une quantité très importante car elle est à la fois intuitive et détermine de nombreuses propriétés du matériau. En particulier, un accroissement de $\overline{u^{2}}$ en fonction de la température plus important que ce qui est indiqué par l'équation 3.166, indique une mise en défaut de l'approximation harmonique et peut être, soit un signe précurseur d'une transition de phase, soit une indication d'un phénomène de relaxation.

L'unité naturelle pour ces déplacements est donc

$$
\frac{\hbar}{2 m \omega_{j}} \text {. }
$$

Les fréquences des phonons sont typiquement de l'ordre du THz. En remplaçant les constantes par leurs valeurs numériques, on obtient

$$
|u|=\sqrt{\frac{1}{3 N} \sum_{j=1}^{3 N} \frac{2 n\left(\omega_{j}\right)+1}{m[\mathrm{uma}] \omega_{j}[\mathrm{THz}]}} \quad 1.8 \AA .
$$

où la masse est à prendre en unités de masse atomique et la fréquence en THz. Nous trouvons donc des valeurs de déplacements extrêmement petits (de l'ordre de $1 / \sqrt{3 N} \AA .63$ ), pour un mode isolé. Si nous assumons que les fréquences des modes sont dans la gamme du THz, alors le déplacement pour

\footnotetext{
62 C'est-à-dire n'étant pas immobiles.

63 Néanmoins, pour les modes acoustiques, le déplacement absolu peut devenir très grand à cause du fait que $\omega$ tend vers zéro pour des petits vecteurs d'onde. Comme on a essayé d'expliquer dans une note en fin de page de la section 3.2, cela ne pose pas un vrai problème pour l'approximation harmonique. La longueur d'onde étant grande, les déplacements relatifs des atomes sont petits comparés à la portée des interactions pour ces modes (Nous ne parlerons pas de tous les phénomènes -certes intéressantsliés à la longue portée du potentiel de Coulomb dans des cristaux ioniques.). En plus, dans un système tridimensionnel, la densité $g(\omega) d \omega$ (voir section 2.1.1) des modes acoustiques $\left(\omega=c k\right.$ ) tend vers zéro comme $\omega^{2}$. Il y a donc de moins en moins de modes, qui contribuent à la somme 3.168 quand $\omega$ baisse.
} 
un atome léger ( $m \approx 10$ uma) sera, à des températures pas trop elevées, une fraction d'un $\AA$. Ce résultat justifie après coup l'approximation harmonique.

\subsection{Densité d'états vibrationnels}

L' Eq. 3.166 peut s'écrire d'une manière plus élégante en introduisant la densité d'états de vibration, c'est-à-dire le nombre de modes vibrationnels par intervalle d'énergie $d E$

$$
g(\omega)=\frac{1}{3 N} \sum_{j=1}^{3 N} \delta\left(\omega-\omega_{j}\right)
$$

La normalisation a été choisie de telle manière que

$$
\int_{0}^{\infty} g(\omega) d \omega=1
$$

En remplaçant l'éq. 3.169 dans l'éq. 3.166 on obtient

$$
\overline{u^{2}}=\frac{\hbar}{2 m} \int_{0}^{\infty} \frac{g(\omega)}{\omega}(2 n(\omega)+1) d \omega .
$$

Les vecteurs propres ont disparu de l'expression. Si les modes se ramollissent, l'amplitude augmente. Dans la limite où $\omega_{j}$ tend vers zéro, nous assistons à une transition de phase de deuxième ordre, la symétrie de la nouvelle phase portant la signature du vecteur propre du mode mou $\vec{e}_{j}$.

Dans un système composé de plusieurs espèces atomiques, ce qui intéresse le scientifique est souvent la densité d'états partielle définie par (voir aussi le cours d'H. Mutka dans cet ouvrage)

$$
F_{\kappa}(\omega)=\frac{1}{3 N_{\kappa}} \sum_{j=1}^{3 N} \sum_{n \in\{\kappa\}} \sum_{\alpha=1}^{3}\left|e_{j}(\alpha, n)\right|^{2} \delta\left(\omega-\omega_{j}\right)=\frac{1}{3 N_{\kappa}} \sum_{j=1}^{3 N} \sum_{n \in\{\kappa\}}\left|\vec{e}_{j}(n)\right|^{2} \delta\left(\omega-\omega_{j}\right) .
$$

La somme portant sur l'indice $n$ inclut l'ensemble des $N_{\kappa}$ atomes appartenant à l'espèce $\kappa$. En partant de $F_{K}(\omega)$, il est possible de déterminer les facteurs de Debye-Waller et les sections efficaces de diffusion (voir le chapitre II).

\subsection{Fonctions thermodynamiques}

Dans la section 2.2, nous avons montré que la grandeur centrale d'un système en équilibre thermodynamique est sa fonction de partition canonique $Z$. Nous avons ensuite vu dans la section 2.3.3 que la fonction de partition appartenant à un seul oscillateur harmonique peut se calculer analytiquement. Nous rappelons ici le résultat

$$
Z=\sum_{n} e^{-\beta E_{n}}=\sum_{n=0}^{\infty} e^{-\beta(n+1 / 2) \hbar \omega}=e^{-(\beta / 2) \hbar \omega} \sum_{n=0}^{\infty} e^{-n \beta \hbar \omega}=\frac{e^{-(\beta / 2) \hbar \omega}}{1-e^{-\beta \hbar \omega}}
$$

Nous allons maintenant généraliser ce résultat à un système harmonique quelconque. En utilisant l'expression 3.153 pour les énergies des états stationnaires, la fonction de partition s'écrit comme

$$
\begin{aligned}
Z & =\sum_{n_{1}, \ldots, n_{3 N}} e^{-\beta E_{n_{1}, \ldots, n_{3} N}} \\
& =\sum_{n_{1}, \ldots, n_{3 N}} e^{-\beta\left(\left(n_{1}+1 / 2\right) \hbar \omega_{1}+\ldots+\left(n_{3 N}+1 / 2\right) \hbar \omega_{3 N}\right)} \\
& =\sum_{n_{1}, \ldots, n_{3 N}} e^{-\beta\left(n_{1}+1 / 2\right) \hbar \omega_{1}} \cdot \ldots \cdot e^{-\beta\left(n_{3 N}+1 / 2\right) \hbar \omega_{3 N}}
\end{aligned}
$$




$$
\begin{aligned}
& =\prod_{j=1, \ldots, 3 N} \sum_{n_{j}=0}^{\infty} e^{-\beta\left(n_{j}+1 / 2\right) \hbar \omega_{j}} \\
& =\prod_{j=1, \ldots, 3 N}\left(e^{-(\beta / 2) \hbar \omega_{j}} \sum_{n_{j}=0}^{\infty} e^{-n \beta \hbar \omega_{j}}\right) \\
& =\prod_{j=1, \ldots, 3 N} \frac{e^{-(\beta / 2) \hbar \omega_{j}}}{1-e^{-\beta \hbar \omega_{j}}} .
\end{aligned}
$$

La fonction de partition $Z$ totale est donc le produit des fonctions de partitions des oscillateurs individuels. ${ }^{64}$ Elle ne dépend des oscillateurs que par l'intermédiaire des fréquences. En particulier elle est indépendante des vecteurs propres. Ceci facilite énormément le calcul des fonctions thermodynamiques. Il suffit de connaître la densité d'états. Comme exemple, nous allons calculer l'énergie interne du système. On part de

$$
\ln Z=\sum_{j=1, \ldots, 3 N} \ln \left[\frac{e^{-(\beta / 2) \hbar \omega_{j}}}{1-e^{-\beta \hbar \omega_{j}}}\right]=\sum_{j=1, \ldots, 3 N}\left[-\frac{\beta}{2} \hbar \omega_{j}-\ln \left(1-e^{-\beta \hbar \omega_{j}}\right)\right] .
$$

Nous convertissons la somme en une integrale, en utilisant la densité d'états

$$
\ln Z=3 N \int d \omega g(\omega)\left[-\frac{\beta}{2} \hbar \omega-\ln \left(1-e^{-\beta \hbar \omega}\right)\right] .
$$

Ce que nous permet de calculer $U$ selon

$$
U=-\frac{d \ln Z}{d \beta}=3 N \frac{\hbar}{2} \int \omega \operatorname{coth}\left(\frac{\hbar \omega}{2 k_{\mathrm{B}} T}\right) g(\omega) d \omega .
$$

Les autres fonctions s'obtiennent de la même manière. Nous ne donnons ici que les résultats. L'énergie libre s'obtient comme

$$
F=3 N k_{\mathrm{B}} T \int \ln \left\{2 \sinh \frac{\hbar \omega}{2 k_{\mathrm{B}} T}\right\} g(\omega) d \omega,
$$

la chaleur spécifique comme

$$
C_{v}=3 N k_{\mathrm{B}} \int\left(\frac{\hbar \omega}{2 k_{\mathrm{B}} T}\right)^{2} \frac{g(\omega)}{\sinh ^{2}\left(\frac{\hbar \omega}{2 k_{\mathrm{B}} T}\right)} d \omega
$$

et l'entropie comme

$$
S=3 N k_{\mathrm{B}} \int_{0}^{\omega_{L}}\left(\frac{\hbar \omega}{2 k_{\mathrm{B}} T} \operatorname{coth} \frac{\hbar \omega}{2 k_{\mathrm{B}} T}-\ln \left[2 \sinh \frac{\hbar \omega}{2 k_{\mathrm{B}} T}\right]\right) g(\omega) d \omega .
$$

Ces résultats montrent à quel point la densité d'états $g(\omega)$ joue un rôle central dans le calcul des quantités thermodynamiques macroscopiques. Des exemples concrets seront présentés quand on parlera de la dynamique du réseau du quartz (figure 36).

\subsection{Vibrations et symétrie des molécules}

Même si nous savons que les fréquences propres ainsi que les modes normaux associés existent, nous sommes loin de pouvoir les déterminer pour un système complexe, comme par exemple un amorphe.

\footnotetext{
${ }^{64}$ Ce résultat est plus général que l'oscillateur harmonique. Comme on pourra facilement le démontrer, $Z=\prod_{j} Z_{j}$ pour tout système qui peut être décomposé en sous-systèmes indépendants.
} 
Même si nous savions diagonaliser la matrice dynamique, il faudrait connaître toutes les constantes de force. Il est ainsi intéressant d'extraire un maximum d'informations sans avoir recours à des calculs explicites. Comme souvent en physique, de telles informations sont extraites des propriétés de symétrie du système : la symétrie établit des relations entre les constantes de force et réduit ainsi les degrés de liberté de la matrice dynamique. Les forces entre deux ensembles d'atomes qui sont images les uns des autres sous une opération de symétrie sont forcément identiques.

Les opérations de symétrie d'une molécule sont les opérations géométriques (réflexion ou réflexion plus translation par rapport à un plan, inversion par rapport à un point et rotation ou rotation puis translation par rapport à un axe), qui ne modifient pas la molécule. Les probabilités de trouver les atomes à des endroits $\left(\vec{r}_{1}, \ldots, \vec{r}_{r}\right)$ exprimées par les fonctions d'onde de la molécule sont donc envoyées sur elles-mêmes par ces opérations. Par conséquent, les fonctions d'ondes elles mêmes doivent refléter les symétries. En particulier, il est évident qu'une opération de symétrie ne pourra renvoyer un état sur un état d'énergie différente. Dans le cas d'un niveau non dégéneré, l'opération de symétrie ajoute un facteur de phase global. ${ }^{65}$ Dans le language de la mécanique quantique, la symétrie se traduit donc par le fait que les fonctions propres de l'Hamiltonien peuvent être classées en utilisant la symétrie du système. Nous avons déjà brièvement mentionné cette possibilité dans la section 2.1.3, quand nous avons classé les fonctions propres des particules libres en utilisant leur impulsion. Ce théorème mathématique est particulièrement utile quand nous avons affaire à des molécules ou, comme nous allons le voir plus loin, à des cristaux.

Les opérations de symétrie forment un groupe. En particulier pour deux opérateurs $G_{a}$ et $\mathrm{G}_{b}$ du groupe il existe un opérateur $G_{c}$ dans le groupe tel que $G_{c}=G_{a} \cdot G_{b}$. Ce groupe possède diverses représentations. Nous ne pouvons pas allez dans les détails de ce qu'est une représentation. Nous nous limitons à indiquer qu'une représentation du groupe est un ensemble d'opérateurs linéaires $\tilde{T}$ agissant dans un espace vectoriel de dimension $s$ et qu'il existe une application entre les deux groupes $\{G\}$ et $\{T\}$, qui respecte la structure du groupe.

$$
T\left(G_{c}\right)=T\left(G_{a}\right) \cdot T\left(G_{b}\right) \quad \text { si } \quad G_{c}=G_{a} \cdot G_{b} \quad \text { et } \quad T(E)=\tilde{I}
$$

avec $E$ l'opération identité du groupe. La représentation est dite de dimension $s$. Les coordonnées des $r$ atomes d'une molécule forment, par exemple, une répresentation de son groupe de symétrie de dimension $3 r$. En général, ces représentations ne sont pas irréductibles, c'est-à-dire qu'on pourra trouver des sous-espace qui donneraient des représentations de dimension plus basse. En d'autres termes, en appliquant toutes les opérations de symétrie, on ne sortirait pas de ces sous-espaces. Un exemple simple est une molécule avec un plan de réflexion comme la molécule d'eau. Si nous construisons une combinaison symétrique des coordonnées des atomes d'hydrogène dans le plan de la molécule, la symétrie sera conservée en appliquant n'importe quelle opération de symétrie de la molécule (voir aussi le cours de F. Guillaume dans cet ouvrage).

Comme les fonctions propres de l'Hamiltonien d'une énergie $E$ donnée forment nécessairement des espaces fermés, elles peuvent être choisies de façon à former des représentations irréductibles du groupe de symétrie. Nous pouvons utiliser les outils de la théorie des groupes pour déterminer les représentations irréductibles qui composent la représentation originale. Soient $m_{j}$ les multiplicités des différentes représentations irréductibles présentes dans la décomposition et $s_{j}$ leurs dimensions respectives. Dans ce cas, $m_{j} \cdot s_{j}$ vecteurs propres se transformeront selon la représentation $\mathrm{IR}_{j}$, avec $\sum_{j} m_{j} \cdot s_{j}=3 N$. Nous pourrons alors, en utilisant uniquement la symétrie, diagonaliser la matrice dynamique en blocs de dimension $m_{j} \times m_{j}$. En langage mathématique, ce constat se manifeste dans

65 La fonction $\psi(\vec{r})$ pourrait par exemple être renvoyée sur $\pm \psi(\vec{r})$ lors d'une réflexion. 
l'existence d'une matrice orthogonale $\tilde{X}$ de dimension $3 N \times 3 N$, qui a la forme suivante :

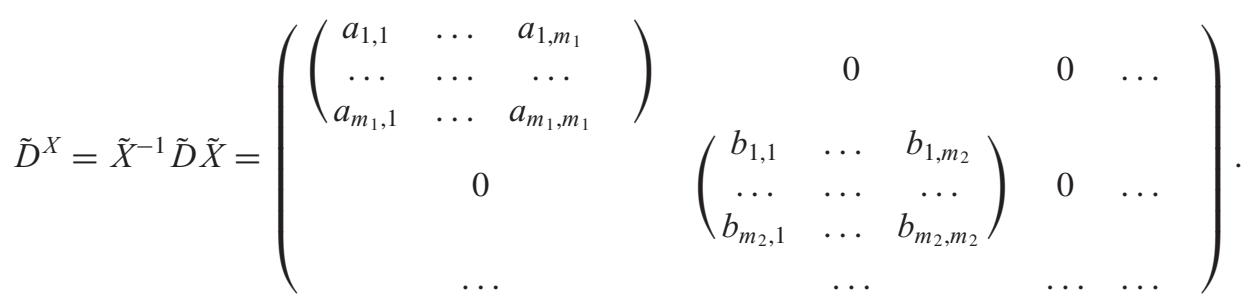

Pour reprendre l'exemple de la molécule d'eau, la symétrie impose deux types de vibrations. On denombre deux vibrations symétriques et une antisymétrique (voir figure 7).

Si la décomposition contient une représentation irréductible de dimension 2 ou plus, le nombre de blocs correspondant est identique à cette dimension. Une dimension 2 signifie que deux modes normaux ont la même énergie par symétrie.

Il est évident que la symétrie peut énormément faciliter la diagonalisation de la matrice dynamique. Même si la diagonalisation en blocs n'est plus vraiment requise par souci de temps de calcul, la classification des modes qui en découle, est un atout considérable pour l'interprétation des résultats. Ce constat vaut en particulier dans le cas où des modèles ou champs de force ont été nécessaires au calcul des fréquences et des vecteurs propres. La caractérisation par symétrie est complètement indépendante des forces d'interaction.

En dépit de son intérêt, il nous est impossible de présenter l'application de la théorie des groupes aux vibrations plus en détail. C'est un sujet assez vaste et d'une complexité certaine. Un bon aperçu de la partie concernant les phonons dans un cristal se trouve dans l'article de Götz Eckold [22].

\subsection{Phonons}

\subsubsection{Symétrie de translation}

La notion de symétrie prend toute son importance quand il s'agit de déterminer les excitations dans un cristal. Un cristal se construit à partir d'un motif qu'on appellera maille primitive. Cette maille se reproduit périodiquement dans l'espace pour former le cristal dans trois dimensions (voir figure 27). Le groupe de symétrie ou groupe d'espace est composé de toutes les opérations qui laissent le cristal inchangé. Il comporte notamment les opérations de symétrie du motif. ${ }^{66}$ Pour l'instant, nous nous limitons à étudier les conséquences physiques de la symétrie translationnelle.

Les translations périodiques peuvent être caractérisées par le vecteur de translation

$$
\vec{l}=l_{1} \vec{a}_{1}+l_{2} \vec{a}_{2}+l_{3} \vec{a}_{3}
$$

où les $\vec{a}_{i} \quad(i=1,2,3)$ représentent les vecteurs primitifs du cristal. Le groupe des translations est un groupe abélien, c'est-à-dire un groupe dont toutes les opérations commutent les unes avec les autres

$$
\tilde{T}_{m} \tilde{T}_{n}=\tilde{T}_{n+m}=\tilde{T}_{n} \tilde{T}_{m}
$$

En d'autres termes, l'ordre dans lequel on effectue les opérations de translation est sans importance pour le résultat final. Ce n'est pas du tout trivial. Par exemple, cette relation n'est pas satisfaite dans le cas des rotations autour d'axes différents.

\footnotetext{
66 La symétrie du motif doit, a priori, être compatible avec la symétrie du réseau. Pour des motifs très symétriques, les forces exercées par les voisins dans le cristal tendent, en général, à reduire la symétrie. Si ces forces dites cristallines sont insuffisantes pour stabiliser la situation, on risque le désordre par frustration. Le motif ne sait pas quelle orientation choisir. Ce sont des sujets de grande actualité dans la recherche.
} 
Comme on peut le démontrer de manière formelle, un groupe abélien ne possède que des représentations unidimensionnelles [23]. Nous devons donc associer à chaque opération de translation des nombres complexes unitaires $\alpha$ pour obtenir ces représentations. ${ }^{67}$ Cela vaut, en particulier, pour les trois translations primitives. Par convention, ces $\alpha$ sont définis par l'intermédiaire de trois nombres réels $k_{i}(i=1,2,3)$. Les translations associées aux trois vecteurs primitifs sont donc représentées sous la forme

$$
\begin{aligned}
\Gamma_{k_{1}, k_{2}, k_{3}}: & \vec{a}_{1} \rightarrow \exp \left(-2 \pi i k_{1}\right), \\
& \vec{a}_{2} \rightarrow \exp \left(-2 \pi i k_{2}\right), \\
& \vec{a}_{3} \rightarrow \exp \left(-2 \pi i k_{3}\right) .
\end{aligned}
$$

Pour une translation arbitraire

$$
\left(\tilde{T}_{l}\right)^{n}=\tilde{T}_{n \cdot l}
$$

Comme les représentations doivent respecter la structure du groupe, cette relation nous amène immédiatement au nombre complexe qui doit représenter une translation $\vec{l}$ quelconque

$$
\Gamma_{k_{1}, k_{2}, k_{3}}: \quad \vec{l} \rightarrow \exp \left(-2 \pi i\left(l_{1} k_{1}+l_{2} k_{2}+l_{3} k_{3}\right)\right) .
$$

Les trois nombres réels $k_{i} \quad(i=1,2,3)$ caractérisent donc la représentation complètement, ce qui justifie entièrement la notation $\Gamma=\Gamma_{k_{1}, k_{2}, k_{3}}$. Pour l'instant, le triplet $\left(k_{1}, k_{2}, k_{3}\right)$ n'est pas encore un vecteur. Nous pouvons néanmoins rendre la notation encore plus compacte

$$
\Gamma_{\vec{k}}: \vec{l} \rightarrow \exp (-i \vec{k} \cdot \vec{l})
$$

si nous exprimons le vecteur $\vec{k}$ dans la base standard du réseau réciproque.

$$
\vec{k}=k_{1} \vec{b}_{1}+k_{2} \vec{b}_{2}+k_{3} \vec{b}_{3}
$$

avec

$$
\begin{aligned}
& \vec{b}_{1}=2 \pi \frac{\vec{a}_{2} \times \vec{a}_{3}}{\vec{a}_{1} \cdot\left(\vec{a}_{2} \times \vec{a}_{3}\right)}, \\
& \vec{b}_{2}=2 \pi \frac{\vec{a}_{3} \times \vec{a}_{1}}{\vec{a}_{1} \cdot\left(\vec{a}_{2} \times \vec{a}_{3}\right)}, \\
& \vec{b}_{3}=2 \pi \frac{\vec{a}_{1} \times \vec{a}_{2}}{\vec{a}_{1} \cdot\left(\vec{a}_{2} \times \vec{a}_{3}\right)} .
\end{aligned}
$$

Pour que le choix des représentations $\Gamma_{\vec{k}}$ soit unique, il faut limiter les vecteurs $\vec{k}$ à la première zone de Brillouin. Nous voudrions attirer l'attention sur le fait qu'en n'utilisant rien d'autre que des arguments de symétrie, nous avons introduit l'espace réciproque et le concept de zone de Brillouin.

Maintenant que nous avons trouvé les représentations irréductibles $\Gamma_{\vec{k}}$ du groupe de translation, nous pouvons déduire que la fonction d'onde d'un état stationnaire de notre Hamiltonien peut être choisie ${ }^{68}$ de manière à se transformer comme

$$
\psi\left(\vec{R}_{1}, \ldots, \vec{R}_{N}\right)=e^{i \vec{k} \cdot \vec{l}} \psi\left(\vec{R}_{1}-\vec{l}, \ldots, \vec{R}_{N}-\vec{l}\right) .
$$

67 Nous allons nous rendre compte de l'utilité de travailler avec des représentations unitaires en voyant les résultats.

68 Il faut ici insister sur le mot "choisir". Comme nous l'avons constaté dans la section 2.1.1, il est tout-à-fait possible de construire des fonctions d'ondes, qui n'ont pas cette propriété dans le cas d'une dégénérescence des niveaux. On peut, par exemple, créer des ondes stationnaires en combinant les fonctions d'ondes pour $\vec{k}$ et $-\vec{k}$. 
La translation de la fonction d'onde introduit donc un facteur de phase. Une fois la représentation c'est-à-dire le vecteur $\vec{k}$ - choisi, cette phase est définie pour toutes les translations $\vec{l} .{ }^{69}$ En particulier, une fonction qui relie la fonction d'onde dans la maille située à l'origine à la fonction d'onde dans une maille située à la distance $\vec{l}$ en multipliant cette dernière par le facteur $\exp (i \vec{k} \cdot \vec{l})$ satisfait cette condition.

\subsubsection{Matrice dynamique dans l'espace réciproque}

Toutes les conséquences de la symétrie translationnelle abordée ici dans le contexte des phonons sont valables dans le cas plus général. Elles s'appliquent, par exemple, aussi aux électrons.

Nous allons d'abord étudier les conséquences de la symétrie de translation dans le cas classique. Considérons la position d'un atome du cristal $\vec{R}(n)(n=1, \ldots N)$. Celle-ci est donnée d'une part par la position de la maille $\vec{l}$ dans laquelle il se trouve, et d'autre part par sa position $\vec{r}_{\kappa}$ à l'intérieur de cette maille (voir figure 27). Nous pouvons donc écrire :

$$
\vec{R}(n)=\vec{R}(\kappa, \vec{l})=\vec{l}+\vec{r}(\kappa),
$$

où $\kappa$ va de 1 à $r$, avec $r$ le nombre d'atomes dans la maille primitive. Nous noterons $\vec{u}(\kappa, \vec{l})$ le déplacement de l'ion situé à la position $\vec{R}(\kappa, \vec{l})$ par rapport à sa position d'équilibre $\vec{R}^{0}(\kappa, \vec{l})$.

Nous nous inspirons du résultat 3.198 pour introduire l' Ansatz

$$
\vec{u}(\kappa, \vec{l} \mid t)=\vec{u}(\kappa \mid \vec{k}) e^{i(\vec{k} \cdot \vec{l}-\omega t)} .
$$

Les vecteurs $\vec{u}(\kappa \mid \vec{k})$ sont censés ne dépendre que de la maille primitive, et le vecteur $\vec{k}$ est choisi dans la première zone de Brillouin.

Les dérivées secondes

$$
\Phi_{\alpha \beta}\left(\begin{array}{cc}
\vec{l} & \vec{l}^{\prime} \\
\kappa & \kappa^{\prime}
\end{array}\right)=\left.\frac{\partial^{2} U}{\partial u_{\alpha}(\kappa, \vec{l}) \partial u_{\beta}\left(\kappa^{\prime}, \vec{l}^{\prime}\right)}\right|_{0}
$$

sont les constantes de force du matériau adaptées à la notation du cristal. Par analogie avec le cas général (pas de symétrie de translation), nous introduisons le vecteur à $3 N$ composantes pondéré par les racines de la masse des ions, et dont les composantes peuvent s'écrire comme

$$
\overrightarrow{\vec{u}}(\kappa, \vec{l} \mid t)=\sqrt{m_{\kappa}} \vec{u}(\kappa, \vec{l} \mid t)=\sqrt{m_{\kappa}} \vec{u}(\kappa \mid \vec{k}) e^{i(\vec{k} \cdot \vec{l}-\omega t)}=\overrightarrow{\vec{u}}(\kappa \mid \vec{k}) e^{i(\vec{k} \cdot \vec{l}-\omega t)} .
$$

Dans cette base, les équations de mouvement classique

$$
m_{\kappa} \ddot{u}_{\alpha}(\kappa, \vec{l} \mid t)=\sum_{\beta, \kappa^{\prime}, \vec{l}^{\prime}} \Phi_{\alpha \beta}\left(\begin{array}{ll}
\vec{l} & \vec{l}^{\prime} \\
\kappa & \kappa^{\prime}
\end{array}\right) u_{\beta}\left(\kappa^{\prime}, \vec{l}^{\prime} \mid t\right)
$$

s'écrivent comme

$$
\ddot{\bar{u}}_{\alpha}(\kappa, \vec{l} \mid t)=-\sum_{\beta, \kappa^{\prime}, \vec{l}^{\prime}} D_{\alpha \beta}\left(\begin{array}{ll}
\vec{l} & \vec{l}^{\prime} \\
\kappa & \kappa^{\prime}
\end{array}\right) \bar{u}_{\beta}\left(\kappa^{\prime}, \vec{l}^{\prime} \mid t\right)
$$

avec, par analogie avec l'équation 3.129

$$
D_{\alpha \beta}\left(\begin{array}{cc}
\vec{l} & \vec{l} \\
\kappa & \kappa^{\prime}
\end{array}\right)=\frac{\Phi_{\alpha \beta}\left(\begin{array}{ll}
\vec{l} & \vec{l}^{\prime} \\
\kappa & \kappa^{\prime}
\end{array}\right)}{\sqrt{m_{\kappa} m_{\kappa^{\prime}}}} .
$$

\footnotetext{
${ }^{69}$ C'est cette dépendance de $\vec{l}$ qui la distingue d'une phase arbitraire et globale, qu'on pourrait toujours rajouter à la fonction d'onde et qui elle ne devrait pas être une fonction de $\vec{l}$.
} 
En utilisant l'Ansatz 3.200 pour $\vec{u}(\kappa, \vec{l} \mid t)$, cette équation se transforme en

$$
\omega^{2} \bar{u}_{\alpha}(\kappa \mid \vec{k})=\sum_{\kappa^{\prime}, \beta} D_{\alpha \beta}\left(\kappa, \kappa^{\prime} \mid \vec{k}\right) \bar{u}_{\beta}\left(\kappa^{\prime} \mid \vec{k}\right),
$$

avec la matrice dynamique $\tilde{D}(\vec{k})$ définie pour chaque $\vec{k}$ de la première zone de Brillouin comme

$$
D_{\alpha \beta}\left(\kappa, \kappa^{\prime} \mid \vec{k}\right)=\sum_{\vec{l}-\vec{l}^{\prime}} D_{\alpha \beta}\left(\begin{array}{ll}
\vec{l} & \vec{l}^{\prime} \\
\kappa & \kappa^{\prime}
\end{array}\right) \exp \left(i \vec{k}\left(\vec{l}^{\prime}-\vec{l}\right)\right) .
$$

$\tilde{D}(\vec{k})$ peut être considérée comme la transformée de Fourier de la matrice des constantes de forces qui, à cause de la périodicité du cristal, ne dépendent que des distances relatives entre atomes.

Le problème initial a finalement pris la forme

$$
\omega_{j}^{2}(\vec{k}) \vec{e}_{j}(\vec{k})=\tilde{D}(\vec{k}) \vec{e}_{j}(\vec{k})
$$

Nous aurons donc trouvé des solutions des équations de mouvement, dès que nous aurons déterminé, pour chaque vecteur $\vec{k}$, les $3 r$ valeurs propres $\omega_{j}^{2}(\vec{k})$ de la matrice dynamique avec leurs vecteurs propres $\vec{e}_{j}(\vec{k})$ associés.

La matrice dynamique $\tilde{D}(\vec{k})$ étant par définition hermitienne

$$
D_{\alpha \beta}\left(\kappa, \kappa^{\prime} \mid \vec{k}\right)=D_{\beta \alpha}^{*}\left(\kappa^{\prime}, \kappa \mid \vec{k}\right)
$$

les valeurs propres $\omega_{j}^{2}$ existent et sont réelles. $\tilde{D}(\vec{k})$ n'étant en général pas symétrique, les vecteurs propres sont complexes. Ils forment une base, qui peut être choisie orthonormale

$$
\vec{e}_{j}^{*}(\vec{k}) \cdot \vec{e}_{j^{\prime}}(\vec{k})=\sum_{\alpha, \kappa=1}^{3, r} e_{j}^{*}(\alpha, \kappa \mid \vec{k}) e_{j^{\prime}}(\alpha, \kappa \mid \vec{k})=\delta_{j, j^{\prime}}
$$

Le fait que le problème classique soit résolu si nous connaissons les valeurs et les vecteurs propres de la matrice dynamique $\tilde{D}(\vec{k})$ pour chaque $\vec{k}$, ou autrement dit, si nous savons diagonaliser cette matrice, est un énorme progrès par rapport à la situation sans symétrie de translation. Il est beaucoup plus facile de diagonaliser une matrice de dimension $3 r \times 3 r$ pour plusieurs valeurs de $\vec{k}$ que de diagonaliser une seule matrice de dimension $3 N \times 3 N$.

Etant donné que le cristal peut toujours être considéré comme une très grosse molécule, et que le problème doit être solvable sans tenir compte des propriétés de symétrie du système, il doit exister une correspondance entre les solutions trouvées ici et celles développées en section 3.4. Autrement dit, il doit être possible d'établir un lien entre les vecteurs propres de la matrice $\tilde{D}$ et ceux des matrices $\tilde{D}(\vec{k})(\vec{k} \in \mathrm{BZ})$ pour une fréquence $\omega$ donnée. Pour un cristal avec $N$ atomes assujettis à des conditions aux limites de Born-von Karman (voir section 2.1.1) il existe $N / r$ valeurs de $\vec{k}$ distinctes dans une zone de Brillouin. Pour chaque $\vec{k}$ nous pouvons construire $3 r$ vecteurs propres

$$
e_{J(j, \vec{k})}(\alpha, i(\kappa, \vec{l}))=\sqrt{\frac{r}{N}} e_{j}(\alpha, \kappa \mid \vec{k}) e^{i(\vec{k} \cdot \vec{l})}
$$

avec $i=i(\kappa, \vec{l})$ dénotant l'atome $\kappa$ dans la maille $\vec{l}$ et $J(j, \vec{k})$ constituant un dénombrement des $j$ et $\vec{k}$. Le facteur $\sqrt{r / N}$ est nécessaire pour la normalisation des vecteurs $e_{J}$ de dimension $3 N$, étant donné que les vecteurs $\vec{e}_{j}(\vec{k})$ de dimension $3 r$ sont normalisés à un. La grande différence avec le cas général est que, pour caractériser un vecteur propre de phonon dans un cristal, il suffit de connaître ses composantes dans une seule maille. Les composantes pour les atomes dans les autres mailles s'en déduisent en appliquant un simple facteur de phase qui est défini par le vecteur d'onde $\vec{k}$. Ce vecteur mérite bien son nom ici étant donné que les solutions correspondent à des ondes progressives. 
Les $3 N$ vecteurs $\vec{e}_{J}$ constituent une base orthonormale

$$
\vec{e}_{J(j, \vec{k})} \cdot \vec{e}_{J^{\prime}\left(j^{\prime}, \vec{k}^{\prime}\right)}=\frac{r}{N} \sum_{\alpha, \kappa=1}^{3, r} e_{j}^{*}(\alpha, \kappa \mid \vec{k}) e_{j^{\prime}}\left(\alpha, \kappa \mid \vec{k}^{\prime}\right) \sum_{\vec{l}} e^{i(\vec{k} \cdot \vec{l})} e^{i\left(\vec{k}^{\prime} \cdot \vec{l}\right)}=\delta_{j, j^{\prime}} \delta_{\vec{k}, \vec{k}^{\prime}}=\delta_{J, J^{\prime}}
$$

Nous pouvons donc décomposer toute solution des équations de mouvement comme

$$
\bar{u}_{\alpha}(\kappa, \vec{l} \mid t)=\sqrt{\frac{r}{N}} \sum_{j=1}^{3 r} \sum_{\vec{k}}^{B Z} Q_{j}(\vec{k}) e_{j}(\alpha, \kappa \mid \vec{k}) e^{-i\left(\vec{k} \cdot \vec{l}-\omega_{j}(\vec{k}) t\right)} .
$$

Les $3 N$ coefficients de développement $Q_{j}(\vec{k})$ sont des nombres complexes dont l'amplitude et la phase sont déterminées par les $3 \mathrm{~N}$ conditions initiales.

Concernant notre question d'équivalence, il reste le problème que les vecteurs propres $\vec{e}_{j}$ de $\tilde{D}$ sont réels tandis que les $\vec{e}_{J(j, \vec{k})}$ sont complexes. On peut y remédier en formant des combinaisons linéaires

$$
\begin{aligned}
& e_{J}^{g}(\alpha, n(\kappa, \vec{l}))=\sqrt{\frac{r}{2 N}}\left(e_{j}(\alpha, \kappa \mid \vec{k}) e^{i(\vec{k} \cdot \vec{l})}+e_{j}^{*}(\alpha, \kappa \mid \vec{k}) e^{-i(\vec{k} \cdot \vec{l})}\right) \\
& e_{J}^{u}(\alpha, n(\kappa, \vec{l}))=-i \sqrt{\frac{r}{2 N}}\left(e_{j}(\alpha, \kappa \mid \vec{k}) e^{i(\vec{k} \cdot \vec{l})}-e_{j}^{*}(\alpha, \kappa \mid \vec{k}) e^{-i(\vec{k} \cdot \vec{l})}\right) .
\end{aligned}
$$

Il faut prendre soin de ne puiser les vecteurs $\vec{k}$ que dans une moitié de la zone de Brillouin. En introduisant la notation $e_{j}(\alpha, \kappa \mid \vec{k})=\left|e_{j}(\alpha, \kappa \mid \vec{k})\right| \exp \left(i \phi_{j}(\alpha, \kappa \mid \vec{k})\right)$, ces relations se transforment en

$$
\begin{aligned}
& e_{J}^{g}(\alpha, i(\kappa, \vec{l}))=\sqrt{\frac{2 r}{N}}\left|e_{j}(\alpha, \kappa \mid \vec{k})\right| \cos \left(\vec{k} \cdot \vec{l}+\phi_{j}(\alpha, \kappa \mid \vec{k})\right), \\
& e_{J}^{u}(\alpha, i(\kappa, \vec{l}))=\sqrt{\frac{2 r}{N}}\left|e_{j}(\alpha, \kappa \mid \vec{k})\right| \sin \left(\vec{k} \cdot \vec{l}+\phi_{j}(\alpha, \kappa \mid \vec{k})\right) .
\end{aligned}
$$

Nous avons donc trouvé une manière alternative d'exprimer les déplacements ponderés

$$
\begin{aligned}
\bar{u}_{\alpha}(\kappa, \vec{l} \mid t)= & \sqrt{\frac{2 r}{N}} \sum_{j=1}^{3 r} \sum_{\vec{k} \in Z B}\left|e_{j}(\alpha, \kappa \mid \vec{k})\right| \\
& \times\left[Q_{j}^{g}(\vec{k}) \cos \left(\vec{k} \cdot \vec{l}+\phi_{j}(\alpha, \kappa \mid \vec{k})\right)+Q_{j}^{u}(\vec{k}) \sin \left(\vec{k} \cdot \vec{l}+\phi_{j}(\alpha, \kappa \mid \vec{k})\right)\right] e^{-i \omega_{j}(\vec{k}) t} .
\end{aligned}
$$

Cette base est beaucoup plus lourde à manipuler. Elle a néanmoins le mérite d'être plus facile à représenter graphiquement. Cela vaut en particulier dès que les vecteurs propres sont réels, comme c'est le cas pour un cristal monoatomique centrosymétrique ${ }^{70}$. Le mouvement se décompose alors simplement en $\sin (\vec{k} \cdot \vec{l})$ et $\cos (\vec{k} \cdot \vec{l})$.

\subsubsection{Diagonalisation de l'Hamiltonien}

Pour faire le lien avec la mécanique quantique, nous nous inspirons de nouveau de l'exemple des deux oscillateurs couplés, traité dans la section 2.1.5. Les changements de variable nécessaires pour

\footnotetext{
${ }^{70}$ C'est-à-dire possédant un centre d'inversion.
} 
diagonaliser la matrice dynamique sont donnés par

$$
\begin{aligned}
Q_{J}=Q_{j}(\vec{k}) & =\sqrt{\frac{r}{N}} \sum_{\alpha, \kappa}^{3, r} e_{j}^{*}(\alpha, \kappa \mid \vec{k}) \sum_{l} e^{-i \vec{k} \cdot \vec{l}} \bar{u}_{\alpha}(\kappa, \vec{l}) \\
& =\sqrt{\frac{r}{N}} \sum_{\alpha, \kappa}^{3, r} \sqrt{m_{\kappa}} e_{j}^{*}(\alpha, \kappa \mid \vec{k}) \sum_{l} e^{-i \vec{k} \cdot \vec{l}} u_{\alpha}(\kappa, \vec{l}), \\
P_{J}=P_{j}(\vec{k}) & =\sqrt{\frac{r}{N}} \sum_{\alpha, \kappa}^{3, r} e_{j}^{*}(\alpha, \kappa \mid \vec{k}) \sum_{l} e^{-i \vec{k} \cdot \vec{l}} \bar{p}_{\alpha}(\kappa, \vec{l}) \\
& =\sqrt{\frac{r}{N}} \sum_{\alpha, \kappa}^{3, r} \frac{e_{j}^{*}(\alpha, \kappa \mid \vec{k})}{\sqrt{m_{\kappa}}} \sum_{l} e^{-i \vec{k} \cdot \vec{l}} p_{\alpha}(\kappa, \vec{l}) .
\end{aligned}
$$

Les coordonnées normales, c'est-à-dire les coordonnées dans la base des vecteurs propres de la matrice dynamique, sont donc les transformées de Fourier des déplacements atomiques ponderés ainsi que de leurs impulsions conjuguées. Ces transformées de Fourier sont calculées pour chaque atome du motif dans l'espace des vecteurs du réseau direct et ensuite projetées sur les vecteurs propres du motif pour le mode consideré. La transformation en coordonnées normales nous permet de construire les opérateurs d'échelle à partir des expressions 3.148 : opérateurs de création

$$
\mathbf{a}_{j}^{+}(\vec{k})=\sqrt{\frac{r}{N}} \sum_{\alpha, \kappa, \vec{l}} e^{i \vec{k} \cdot \vec{l}} e_{j}(\alpha, \kappa \mid \vec{k}) \cdot\left[\sqrt{\frac{m_{\kappa} \omega_{j}(\vec{k})}{2 \hbar}} \mathbf{u}_{\alpha}(\kappa, \vec{l})-i \sqrt{\frac{1}{2 \hbar m_{\kappa} \omega_{j}(\vec{k})}} \mathbf{p}_{\alpha}(\kappa, \vec{l})\right]
$$

et d'annihilation

$$
\mathbf{a}_{j}(\vec{k})=\sqrt{\frac{r}{N}} \sum_{\alpha, \kappa, \vec{l}} e^{-i \vec{k} \cdot \vec{l}} e_{j}^{*}(\alpha, \kappa \mid \vec{k}) \cdot\left[\sqrt{\frac{m_{\kappa} \omega_{j}(\vec{k})}{2 \hbar}} \mathbf{u}_{\alpha}(\kappa, \vec{l})+i \sqrt{\frac{1}{2 \hbar m_{\kappa} \omega_{j}(\vec{k})}} \mathbf{p}_{\alpha}(\kappa, \vec{l})\right] .
$$

Ces opérateurs sont, d'une certaine manière, les transformées de Fourier des opérateurs de création et d'annihilation locaux $\mathbf{a}^{+}(\kappa, \vec{l})$ et $\mathbf{a}(\kappa, \vec{l})$.

Comme dans le cas des vibrations, nous pouvons inverser ces relations et obtenir les opérateurs de déplacement et d'impulsion dans les trois directions $(\alpha=x, y, z)$

$$
\begin{aligned}
\mathbf{u}_{\alpha}(\vec{l}, \kappa) & =\sqrt{\frac{r}{N}} \sum_{j, \vec{k}} \sqrt{\frac{\hbar}{2 m_{\kappa} \omega_{j}(\vec{k})}}\left[e_{j}(\alpha, \kappa \mid \vec{k}) e^{i \vec{k} \cdot \vec{l}} \mathbf{a}_{j}(\vec{k})+e_{j}^{*}(\alpha, \kappa \mid \vec{k}) e^{-i \vec{k} \cdot \vec{l}} \mathbf{a}_{j}^{+}(\vec{k})\right] \\
& =\sqrt{\frac{r}{N}} \sum_{j, \vec{k}} \sqrt{\frac{\hbar}{2 m_{\kappa} \omega_{j}(\vec{k})}} e_{j}(\alpha, \kappa \mid \vec{k})\left(\mathbf{a}_{j}(\vec{k})+\mathbf{a}_{j}^{+}(-\vec{k})\right) e^{i \vec{k} \cdot \vec{l}}
\end{aligned}
$$

et de leur moment cinétique

$$
\begin{aligned}
\mathbf{p}_{\alpha}(\vec{l}, \kappa) & =-i \sqrt{\frac{r}{N}} \sum_{j, \vec{k}} \sqrt{\frac{\hbar \omega_{j}(\vec{k}) m_{\kappa}}{2}}\left[e_{j}(\alpha, \kappa \mid \vec{k}) e^{i \vec{k} \cdot \vec{l}} \mathbf{a}_{j}(\vec{k})-e_{j}^{*}(\alpha, \kappa \mid \vec{k}) e^{-i \vec{k} \cdot \vec{l}} \mathbf{a}_{j}^{+}(\vec{k})\right] \\
& \left.=-i \sqrt{\frac{r}{N}} \sum_{j, \vec{k}} \sqrt{\frac{\hbar \omega_{j}(\vec{k}) m_{\kappa}}{2}} e_{j}(\alpha, \kappa \mid \vec{k})\left(\mathbf{a}_{j}(\vec{k})-\mathbf{a}_{j}^{+}(-\vec{k})\right]\right) e^{i \vec{k} \cdot \vec{l}}
\end{aligned}
$$


en fonction des opérateurs de création et d'annihilation. Les deuxièmes égalités dans chaque relation utlisent $\vec{e}_{j}(\vec{k})=\vec{e}_{j}(-\vec{k})$. Comme nous le verrons plus loin, elles ne sont donc valables que lorsque tout atome du cristal est un centre d'inversion, ce qui est toujours le cas pour un cristal monoatomique.

Remplaçant les expressions 3.223 dans l'Hamiltonien 3.122, nous obtenons, en parfaite analogie avec l'expression 3.151 pour les vibrations,

$$
\mathbf{H}=\sum_{\vec{k}} \sum_{j=1}^{3 r} \hbar \omega_{j}(\vec{k})\left(\mathbf{a}_{j}^{+}(\vec{k}) \mathbf{a}_{j}(\vec{k})+\frac{1}{2}\right),
$$

L'Hamiltonien est ainsi diagonalisé.

Nous voudrions conclure cette section avec la remarque suivante : la diagonalisation de l'Hamiltonien, qui est une forme bilinéaire des opérateurs de position et d'impulsion, est tout-à-fait possible sans passer par les solutions des équations classiques de mouvement. Il suffit d'appliquer les théorèmes de l'algèbre linéaire pour effectuer le découplage des variables par un changement de base orthonormale approprié. L'approche choisie dans ce cours permet néanmoins de mieux saisir les concepts physiques qui se cachent derrière les formules.

\subsubsection{Caractérisation des modes d'un cristal}

Nous allons maintenant résumer ces résultats en acceptant volontiers le risque de répéter des choses déjà dites.

- La matrice dynamique pour un $\vec{k}$ donné est de dimension $3 r \times 3 r$ et possède $3 r$ valeurs propres. Au lieu de devoir trouver une solution à un système couplé à $3 N$ dimensions, le problème peut se réduire à trouver $3 r$ valeurs propres à chaque matrice dynamique $\tilde{D}(\vec{k})$.

- Il suffit de puiser les vecteurs $\vec{k}$ dans la première zone de Brillouin (BZ). Tout autre vecteur ne produira que des solutions équivalentes.

- En utilisant des conditions aux limites de Born-von Karman (voir section 2.1.1), on trouve qu'il y a $N / r$ vecteurs

$$
\vec{k}=\left(n_{x} \frac{2 \pi}{L}, n_{y} \frac{2 \pi}{L}, n_{z} \frac{2 \pi}{L}\right), \quad n_{x}, n_{y}, n_{z}=-\frac{1}{2}\left(\frac{N}{r}\right)^{\frac{1}{3}}+1 \ldots-1,0,1, \ldots \frac{1}{2}\left(\frac{N}{r}\right)^{\frac{1}{3}}
$$

dans la première zone de Brillouin. ${ }^{71}$ Trouver $3 r$ valeurs propres pour $N / r$ vecteurs $\vec{k}$ correspond bien à résoudre un système de $3 N$ équations différentielles couplées.

- La matrice dynamique est hermitienne ${ }^{72}$

$$
D_{\alpha \beta}\left(\kappa, \kappa^{\prime} \mid \vec{k}\right)=D_{\beta \alpha}^{*}\left(\kappa^{\prime}, \kappa, \mid \vec{k}\right)
$$

Les valeurs propres $\omega_{j}^{2}$ sont donc réelles.

- Il suit directement de la définition de la matrice dynamique que

$$
D_{\alpha \beta}\left(\kappa \kappa^{\prime} \mid \vec{k}\right)=\sum_{l-l^{\prime}} \frac{\Phi_{\alpha \beta}\left(\begin{array}{cc}
\vec{l} & \vec{l}^{\prime} \\
\kappa & \kappa^{\prime}
\end{array}\right)}{\sqrt{m_{\kappa} m_{\kappa}^{\prime}}} e^{i \vec{k}\left(\vec{l}^{\prime}-\vec{l}\right)}=D_{\alpha, \beta}^{*}\left(\kappa \kappa^{\prime} \mid(-\vec{k})\right)
$$

Cela implique pour les valeurs propres

$$
\omega_{j}^{2}(-\vec{k})=\omega_{j}^{2}(\vec{k})
$$

\footnotetext{
71 Pour ne pas inutilement encombrer la notation, nous supposons avoir affaire à un réseau cubique.

72 C'est une propriété de tout opérateur en mécanique quantique représentant une observable.
} 
Il est donc suffisant de retenir la racine positive $\omega_{j}(\vec{k}) .{ }^{73}$

- Si nous nous imaginons que le motif correspond au cristal, il ne reste que le vecteur $\vec{k}=0$. La matrice dynamique pour ce $\vec{k}$ correspond à celle (éq. 3.129) qu'on a trouvée pour les vibrations dans une molécule de cette forme et de cette taille. Le cristal devient, en d'autres termes, une molécule immense.

- Les vecteurs propres $\vec{e}_{j}(\vec{k})$ de dimension $3 r$ décrivent, pour chaque $\vec{k}$ et pour chaque valeur propre $\omega_{j}$, un motif de déplacement dans la maille primitive. Les $\vec{e}_{j}(\vec{k})$ sont en général complexes. Ils décrivent à la fois les relations de position et de phase de ce motif. Ce fait a été démontré lors de la discussion de la chaîne linéaire.

- Ceci nous amène directement au concept de surface de dispersion. Pour chaque valeur de $\vec{k}$ nous avons $3 r$ fréquences $\omega_{j}(\vec{k})$, qui sont liées à $3 r$ modes de vibrations caractérisés chacun par un vecteur propre $\vec{e}_{j}(\vec{k})$. Le vecteur $\vec{k}$ étant un paramètre quasi-continu ${ }^{74}$ les fréquences $\omega_{j}(\vec{k})$ forment $3 r$ surfaces de dispersion de dimension trois (dans l'espace à quatre dimensions $\left.\left(\omega, k_{x}, k_{y}, k_{z}\right)\right)$. Si on coupe ces surfaces le long d'une direction, on obtient $3 r$ courbes de dispersion.

- Les courbes de dispersion possèdent par définition la périodicité du réseau réciproque. Cela implique que les surfaces de dispersion sont plafonnées vers le haut, c'est-à-dire il existe une fréquence $\omega_{j}$ maximale pour chaque surface $j$.

- La symétrie, autre que translationnelle, du cristal peut imposer que plusieurs fréquences, ou même deux courbes de dispersion le long d'une direction de haute symétrie, doivent coïncider. Ce phénomène est connu sous le nom de dégénérescence. Il est dû au fait que la symétrie lie les ions entre eux et de cette façon établit des relations entre les éléments correspondants de la matrice dynamique.

- La symétrie, autre que translationnelle, ne crée pas seulement des dégénérescences, mais impose aussi ses conditions aux vecteurs propres. Ceci se voit par exemple quand nous sommes confrontés à un cristal dont chaque atome est un centre d'inversion. Chaque atome étant invariant sous l'action de l'inversion, les vecteurs propres peuvent être choisis de manière à remplir la condition

$$
\vec{e}_{j}(\vec{k})=\vec{e}_{j}(-\vec{k})
$$

Mais, comme en général

$$
\vec{e}_{j}(\vec{k})=\vec{e}_{j}^{*}(-\vec{k})
$$

il suit que

$$
\vec{e}_{j}(\vec{k})=\vec{e}_{j}^{*}(\vec{k}) .
$$

Les vecteurs propres peuvent donc être choisis réels. Un motif de déplacement typique nécessitant une description complexe est une hélice. On pense à la lumière polarisée circulairement. Si le cristal a un centre d'inversion, l'hélice droite est dégénérée avec une hélice gauche. En combinant les deux mouvements correctement, on obtient deux modes polarisés linéairement.

- Dans le langage de la théorie des groupes, les courbes de dispersion dans une direction $\vec{k}$ donnée peuvent être classées selon les représentations du groupe de symétrie du vecteur $\vec{k}$, constitué des opérations de symétrie du cristal qui laissent le vecteur $\vec{k}$ invariant (à un vecteur $\vec{G}$ du réseau

\footnotetext{
73 On peut montrer que $\omega_{j}^{2}(\vec{k})$ doit être positive si tous les ions possèdent bien des positions d'équilibre.

74 Nous pouvons rendre arbitrairement grand le nombre de $\vec{k}$ par unité de $n m^{-3}$ en augmentant les longueurs $L_{x}, L_{y}$ et $L_{z}$ utilisées pour les conditions aux limites Born-von Karman.
} 

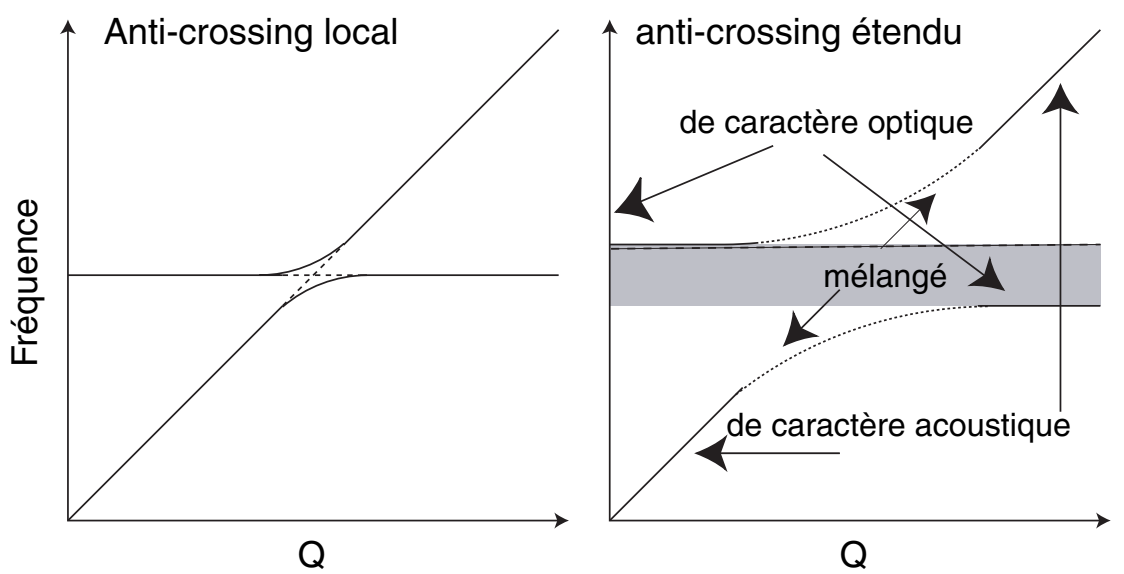

Figure 28. Explication schématique du croisement de deux branches de dispersion, l'une acoustique et l'autre optique. Si les deux courbes de dispersion partagent la même symétrie, la probabilité pour qu'elles se croisent sans se repousser tend vers zéro. Néanmoins, le degré de répulsion dépendra des détails du système. Il pourra varier d'une répulsion faible et donc très locale dans l'espace réciproque à une répulsion forte et donc étendue. Si la répulsion est faible, les modes vont garder leur caractère d'origine en dehors de la région d'intersection. Par exemple, la branche basse aura un vecteur de polarisation acoustique jusqu'au point d'intersection et de plus en plus optique au delà. L'inverse sera vrai pour la branche haute.

réciproque près). Pour le vecteur $\vec{k}=0$, ce groupe correspond au groupe de symétrie total du cristal. C'est au centre de zone, appelé aussi point $\Gamma$, que les effets de symétrie sont les plus prononcés.

- Si $\vec{k}$ pointe dans une direction arbitraire, il n’y a pas de symétrie spéciale et donc pas de dégénérescence requise par la symétrie. Toute dégénérescence serait donc accidentelle. En d'autres termes, les constantes de forces seraient telles que pour un point $\vec{k}$, deux modes coincident en fréquence mais que le moindre changement de ces forces, permis par la symétrie, lèverait cette dégénérescence. Dans ce cas, la probabilité de cette dégénérescence est pratiquement nulle. ${ }^{75}$ Les branches de dispersion se "repoussent" et on parle d'anti-croisement (voir figure 28). L'anti-croisement est la situation normale partout dans la zone de Brillouin en dehors des directions ou points de haute symétrie. Les surfaces de dispersion ne s'interpénètrent donc pas en dehors de ces directions. Ce fait nous permet de dénombrer clairement les surfaces de dispersion, c'est-à-dire de parler de la surface la plus basse, des surfaces intermédiaires et de la surface la plus haute en fréquence.

- Les modes, pour des vecteurs $\vec{k}$ qui sont reliés par des opérations de symétrie, ne sont pas indépendants. Il suffit donc d'étudier qu'une des parties de la zone de Brillouin qui s'avèrent équivalentes par symétrie. Pour un système cubique sans symétrie supplémentaire, cette partie correspond à un huitième du cube.

- A cause des termes "auto" (voir éq. 3.128)

$$
\sum_{\kappa^{\prime}, \beta, \vec{l}^{\prime}} \Phi_{\alpha \beta}\left(\begin{array}{ll}
\vec{l} & \vec{l}{ }^{\prime} \\
\kappa & \kappa^{\prime}
\end{array}\right)=0
$$

\footnotetext{
75 Voir la référence [24] pour une discussion mathématique de ce phénomène aussi connu sous le nom de principe de von Neumann et qui stipule que "tout ce que la symétrie permet a normalement lieu".
} 
Cette propriété suffit pour démontrer que la matrice dynamique en centre de zone possède 3 valeurs propres qui sont nulles. Les vecteurs propres associés sont de la forme

$$
\begin{aligned}
& e_{\mathrm{acc}}^{x}(\vec{k}=0) \propto\left(\left(\frac{1}{\sqrt{M_{1}}}, 0,0\right),\left(\frac{1}{\sqrt{M_{2}}}, 0,0\right), \ldots,\left(\frac{1}{\sqrt{M_{r}}}, 0,0\right)\right), \\
& e_{\mathrm{acc}}^{y}(\vec{k}=0) \propto\left(\left(0, \frac{1}{\sqrt{M_{1}}}, 0\right),\left(0, \frac{1}{\sqrt{M_{2}}}, 0\right), \ldots,\left(0, \frac{1}{\sqrt{M_{r}}}, 0\right)\right), \\
& e_{\mathrm{acc}}^{z}(\vec{k}=0) \propto\left(\left(0,0, \frac{1}{\sqrt{M_{1}}}\right),\left(0,0, \frac{1}{\sqrt{M_{2}}}\right), \ldots,\left(0,0, \frac{1}{\sqrt{M_{r}}}\right)\right) .
\end{aligned}
$$

Ces vecteurs correspondent à des déplacements de l'ensemble des ions dans les trois directions de l'espace. Parce que le système dans son intégralité ne rencontre pas de forces de rappel sans champs macroscopiques externes, les fréquences doivent être nulles. Ce n'est plus le cas si le système est greffé sur une surface. Pour des petits $k$, ces modes sont connectés aux trois modes acoustiques $\omega(k) \propto k$. La raideur de la pente dépendra de la direction.

- Si nous choisissons une direction particulière, seul un mode acoustique aura les déplacements dans la direction de $\vec{k}$. Si cette direction coïncide, par exemple, avec $x$ ce sera le mode numéro 1 de l'expression 3.234 dans le système. On l'appellera mode acoustique longitudinal. Pour les deux autres modes, les ions se déplacent selon $y$ ou $z$, c'est-à-dire perpendiculairement à la direction du vecteur $\vec{k}$. On les appellera modes acoustiques transverses.

- Etant donné que les vecteurs propres sont orthogonaux, tous les autres modes correspondent à des mouvements relatifs des ions. Si un de ces modes possédait une fréquence égale à zéro, cela voudrait dire qu'une partie du cristal peut être déplacée par rapport au reste sans perte d'énergie. Un tel cristal serait mécaniquement instable et devrait relaxer. Nous ne considérons pas cette situation. En particulier, en centre de zone, tous les modes sauf les trois acoustiques ont des fréquences différentes de zéro. Ces fréquences se trouvent normalement dans la gamme de la lumière infrarouge. On les appellera modes optiques.

- Quand un mode optique de basse fréquence croise un mode acoustique dans une direction arbitraire, les deux modes vont, d'après le principe de von Neumann qu'on a énoncé plus haut, se repousser. Mais attention : selon le degré d'hybridation entre les deux modes, ils vont préserver ou non leur caractère, donné par leur vecteur propre (voir figure 28).

- Pour les modes acoustiques transverses, le vecteur propre est perpendiculaire au vecteur $\vec{k}$. Selon l'expression 3.223, ceci entraîne que tous les opérateurs d'impulsion des ions sont perpendiculaires au vecteur $\vec{k}$. Nous apprenons donc que la grandeur $\hbar \vec{k}$ ne peut pas être associée à l'impulsion comme nous pouvions le faire dans le cas de la particule libre (voir section 2.1.3). En dépit de ce fait, le vecteur $\vec{k}$ figure souvent dans les lois de conservation dite de "la quantité de mouvement". Nous discuterons ces lois lors de la discussion de la diffusion des neutrons par les excitations du réseau (se référer au chapitre III).

- La périodicité des surfaces de dispersion implique qu'elles ont une pente nulle en bord ainsi qu'en centre de zone

$$
\vec{\nabla} \omega_{j}(\vec{k})=0
$$

sauf dans les cas exceptionnels où elles seraient dégénérées. La densité d'états étant la projection de toutes les surfaces de dispersion sur l'axe d'énergie, il est évident que les contributions fortes seront obtenues pour des régions de $\vec{k}$ dans lesquelles la fréquence ne change que très peu avec $\vec{k}$. Mathématiquement, ceci s'exprime par la formule

$$
g(\omega)=\sum_{j} \int_{S_{j}(\omega)} \frac{d S}{(2 \pi)^{3}} \frac{1}{\left|\vec{\nabla} \omega_{j}(\vec{k})\right|},
$$




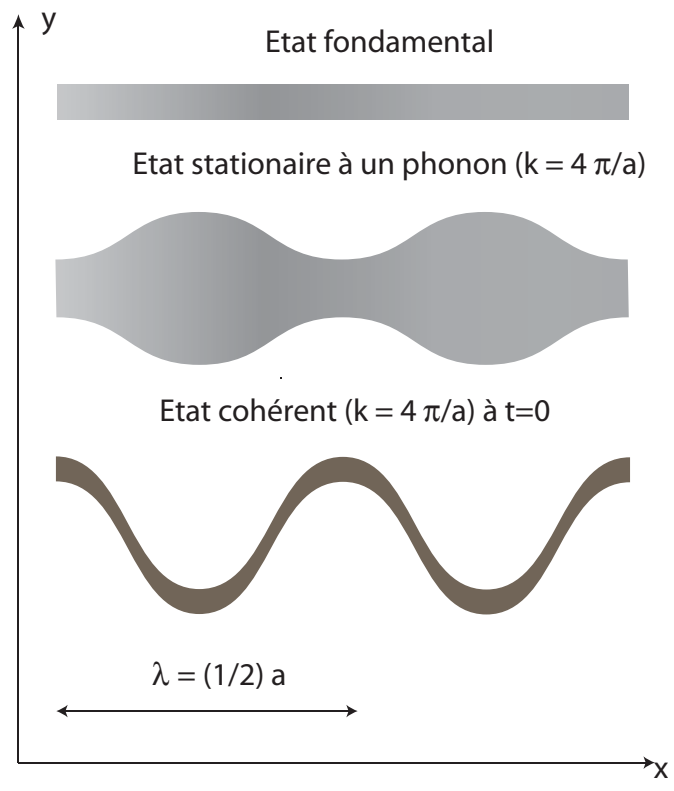

Evolution avec le temps de l'état cohérent

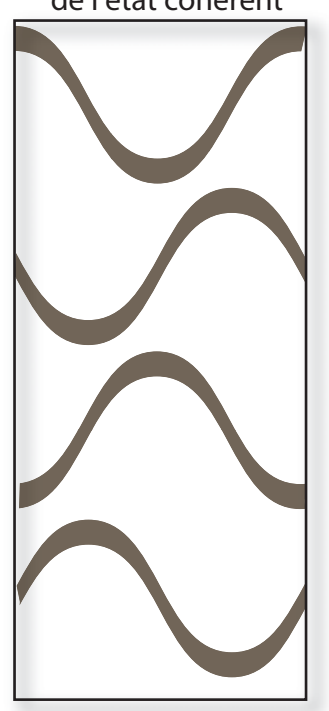

Figure 29. Présentation schématique des différents types d'états pour une chaîne monoatomique transverse. Dans l'état fondamental, la probabilité de trouver une particule est, pour tous les atomes, une gaussienne dont la largeur est régie par la raideur du ressort, comme pour les oscillateurs couplés. Pour un état à un seul phonon, la fonction d'onde est toujours stationnaire. Elle est en plus centrée pour tous les atomes aux positions d'équilibre. La forme d'onde du mode se reflète dans la variation de la fonction d'onde avec la position atomique. Un état cohérent obtenu en superposant un grand nombre de phonons selon l'expression 3.251 aura une fonction d'onde correspondant à une onde classique. Selon le type de superposition elle sera soit propagatrice soit stationnaire (l'exemple montré ici correspond à une onde propagatrice). Les probabilités de trouver les atomes seront centrées aux points de déplacement classiques. Ils évoluent avec le temps comme une onde classique. Plus il y a de modes présents dans la superposition et plus cette analogie entre image classique et fonction d'onde sera justifiée.

qui donne la contribution d'une surface de dispersion $S_{j}(\omega)$ à la densité d'états. ${ }^{76}$ L'intégrale doit être évaluée sur toutes les parties de la zone de Brillouin pour lesquelles $\omega_{j}(\vec{k})=\omega$. Les points pour lesquels $\vec{\nabla} \omega_{j}(\vec{k})$ est égale à zéro constituent des singularités, qui sont néanmoins intégrables à trois dimensions. Elles sont connues sous le nom de singularités de Van Hove. Il est évident que les singularités apparaissant en bord de zone vont dominer la densité d'états puisque la surface d'intégration y est maximale. Les singularités en centre de zone ne concernent normalement qu'une petite partie de l'espace réciproque et ne contribuent donc que faiblement à la densité d'états ${ }^{77}$. Les singularités de Van Hove et la dépendance en $\omega^{2}$ pour les modes acoustiques dans un système tridimensionnel définissent déjà bien la forme de la densité d'états dans des systèmes simples.

\subsubsection{Les fonctions propres}

Jusqu'ici, nous avons parlé des fréquences et des vecteurs propres de la matrice dynamique. Il nous reste à caractériser les états propres de 1'Hamiltonien 3.225, que nous reproduisons ici pour faciliter la

\footnotetext{
76 Nous travaillons ici dans la limite $L \rightarrow \infty$ ce qui nous permet de remplacer la somme sur $\vec{k}$ par une intégrale.

77 Ce n'est plus le cas pour un système de basse dimension. Les contributions en centre de zone et en bord de zone contribuent de la même façon pour un système unidimensionnel.
} 
discussion :

$$
\mathbf{H}=\sum_{\vec{k} \in Z B} \sum_{j=1}^{3 r} \hbar \omega_{j}(\vec{k})\left(\mathbf{a}_{j}^{+}(\vec{k}) \mathbf{a}_{j}(\vec{k})+\frac{1}{2}\right) .
$$

La différence par rapport à l'opérateur plus général 3.151, qui ne prend pas en compte la symétrie de translation, réside dans le fait que les fonctions propres s'obtiennent ici dans une base d'ondes planes. Pour un vecteur d'onde $\vec{k}$ donné, la fonction d'onde associée s'écrit comme

$$
\left|\psi_{n_{1}(\vec{k}), \ldots, n_{3 r}(\vec{k})}\right\rangle \equiv\left|n_{1}(\vec{k}), \ldots, n_{3 r}(\vec{k})\right\rangle=\prod_{j=1}^{3 r} \frac{\left(\hat{a}_{j}^{+}\right)^{n_{j}(\vec{k})}}{\sqrt{n_{j}(\vec{k}) !}}|0, \ldots, 0\rangle .
$$

La base complète des fonctions d'onde s'obtient par produit direct des fonctions

$$
\left|n_{1}(\vec{k}), \ldots, n_{j}(\vec{k})\right\rangle
$$

pris sur tous les vecteurs d'onde $\vec{k}$. Le nombre entier

$$
n_{j}(\vec{k})=\left\langle\mathbf{a}_{j}^{+}(\vec{k}) \mathbf{a}_{j}(\vec{k})\right\rangle
$$

indique pour chaque oscillateur, caractérisé par le vecteur propre $\vec{k}$ et l'indice de surface de dispersion $j$, le niveau d'excitation dans lequel il se trouve. L'énergie totale du système dans une superposition d'états stationnaires est donc donnée par

$$
E=\sum_{\vec{k}}^{B z} \sum_{j=1}^{3 r} \hbar \omega_{j}\left(n_{j}(\vec{k})+\frac{1}{2}\right) .
$$

Le langage devient vite encombrant si on est en permanence obligé d'évoquer les niveaux d'excitation $n_{j}(\vec{k})$ des oscillateurs. Pour cette raison, on utilise universellement une terminologie empruntée à la théorie des champs. Un oscillateur est un réservoir capable de recevoir des excitations appelées phonons. L'état d'excitation est exprimé en termes d'occupation $n_{j}(\vec{k})$, c'est-à-dire en fonction du nombre des phonons présents. Au lieu de dire que "le mode $(j, \vec{k})$ se trouve dans un état excité $n_{j}(\vec{k})$ ", nous parlons de "la présence de $n_{j}(\vec{k})$ phonons de type $(j, \vec{k})$ dans le cristal". Le terme phonon souligne l'analogie avec le champ électromagnétique dont les excitations fondamentales sont les photons. Créer un phonon équivaut à augmenter l'état correspondant d'un niveau. Annihiler un phonon équivaut à baisser l'état correspondant d'un niveau. Il faut néanmoins être prudent et ne pas oublier que chaque état excité a sa propre personnalité décrite en terme des fonctions d'onde de l'oscillateur harmonique dans les coordonnées normales $\mathbf{Q}_{J}$. Quand on crée un phonon à partir de l'état fondamental, on ne produit pas le même changement dans le système que quand on crée le même phonon en partant d'un état déjà excité.

Caractérisons un peu plus les phonons.

- Un phonon est par définition étendu sur tout le cristal. Cela ne veut pas dire que tous les ions doivent participer au mouvement. Comme nous l'avons vu dans le cas de la chaîne linéaire, il y a bien des vecteurs propres qui comportent des zéros pour certains ions. Le taux de participations défini par l'expression 3.163 reste néanmoins toujours très élevé et ne peut pas descendre en dessous de $\frac{1}{r}$.

- Un atome dont le vecteur propre est zéro pour un mode donné n'est pas figé dans sa position d'équilibre. Cela serait en contradiction avec les règles fondamentales de la mécanique quantique. L'atome se trouve dans un état fondamental, qui est caractérisé par les fluctuations quantiques. 
- Un phonon correspond à une onde plane de la forme

$$
\psi(\vec{r}, t)=A(\cos (\vec{r} \cdot \vec{k}-\omega t)+i \sin (\vec{r} \cdot \vec{k}-\omega t))=A e^{i(\vec{k} \cdot \vec{r}-\omega t)}
$$

Pour obtenir des modes stationnaires, il faut superposer les phonons à $\vec{k}$ avec ceux à $-\vec{k}$. On aurait pu diagonaliser dès le début l'Hamiltonien dans la base de ces modes. Néanmoins les ondes planes et leur représentation complexe rendent le formalisme beaucoup plus simple, comme nous l'avons démontré.

- Un phonon correspond à un état stationnaire de l'Hamiltonien. Par conséquent les probabilités de présence des ions n'évoluent pas dans le temps et sont centrées sur leurs positions d'équilibre (voir figure 29). Un phonon ne doit pas être confondu avec une oscillation classique.

- Pour obtenir des fonctions qui évoluent dans le temps, il faut superposer des phonons de différentes énergies. Les paquets d'ondes créés à partir des phonons s'élargiront avec le temps.

\subsection{Localisation}

Pour un grand nombre de problèmes physiques, et en particulier ceux du transport, il est intéressant de poursuivre la question de la localisation d'un mouvement. Nous restons ici avec un système à symétrie de translation, c'est-à-dire que nous ne traiterons pas la localisation (certainement intéressante) dûe à des défauts de structure ou de masse. Il faut distinguer deux cas: la localisation d'un motif de vibration ou la localisation du mouvement d'un ion.

Regardons d'abord l'exemple d'un cristal moléculaire. Dans le paragraphe dédié au cas de la chaîne linéaire à ressorts alternés (section 3.3.3), nous avons vu que dans un système moléculaire il existe des modes optiques plats et que les vecteurs propres de ces modes ne dépendent que très peu du vecteur $\vec{k}$ (voir section 3.3.3). Les arguments peuvent être repris pour le cas général. Les fréquences et les vecteurs propres des modes internes d'un cristal moléculaire ne dépendent que très peu de $\vec{k}$. Dans le cas extrême nous pouvons supposer que $\forall \vec{k}$

$$
\begin{aligned}
\omega_{j}(\vec{k}) & =\omega_{j}, \\
e_{j}(\alpha, \kappa \mid \vec{k}) & =e_{j}(\alpha, \kappa) .
\end{aligned}
$$

Nous avons donc affaire à un ensemble de modes hautement dégénérés. A l'intérieur de cet ensemble nous sommes libres d'effectuer des changements de base sans risque de compromettre la diagonalisation de l'Hamiltonien. En partant de l'expression 3.220 nous pouvons construire des nouvelles variables

$$
\begin{aligned}
& \mathbf{Q}_{j}(\vec{l})=\sum_{\vec{k} \in B Z} \mathbf{Q}_{j}(\vec{k}) e^{i \vec{k} \cdot \vec{l}}=\sqrt{\frac{r}{N}} \sum_{\vec{k}}^{B Z} \sum_{\alpha, \kappa}^{3, r} \sqrt{m_{\kappa}} e_{j}^{*}(\alpha, \kappa \mid \vec{k}) \sum_{l^{\prime}} e^{\left.-i \vec{k} \cdot \overrightarrow{l^{\prime}}-\vec{l}\right)} \mathbf{u}_{\alpha}\left(\kappa, \vec{l}^{\prime}\right) \\
& =\sqrt{\frac{r}{N}} \sum_{\alpha, \kappa}^{3, r} \sqrt{m_{\kappa}} e_{j}^{*}(\alpha, \kappa) \sum_{l^{\prime}} \mathbf{u}_{\alpha}\left(\kappa, \vec{l}^{\prime}\right) \sum_{\vec{k}}^{B Z} e^{-i \vec{k} \cdot\left(\overrightarrow{l^{\prime}}-\vec{l}\right)} \\
& =\sqrt{\frac{r}{N}} \sum_{\alpha, \kappa}^{3, r} \sqrt{m_{\kappa}} e_{j}^{*}(\alpha, \kappa) \sum_{l^{\prime}} \mathbf{u}_{\alpha}\left(\kappa, \vec{l}^{\prime}\right) \delta\left(\vec{l}-\vec{l}^{\prime}\right) \\
& =\sqrt{\frac{r}{N}} \sum_{\alpha, \kappa}^{3, r} \sqrt{m_{\kappa}} e_{j}^{*}(\alpha, \kappa) \mathbf{u}_{\alpha}(\kappa, \vec{l}) \text {. }
\end{aligned}
$$

Les variables $\mathbf{Q}_{j}(\vec{l})$ sont locales, c'est-à-dire qu'elles s'obtiennent à partir de combinaisons linéaires des coordonnées des atomes d'une seule maille $\vec{l}$. Etant donné que les mailles sont au nombre de $N / r$, 
ces variables forment une base d'un sous-espace de Hilbert de dimension $N / r$. L'Hamiltonien restant forcément diagonalisé dans cette base, nous pouvons créer et annihiler des excitations quantiques en appliquant les opérateurs

$$
\begin{aligned}
& \mathbf{a}_{j}^{+}(\vec{l})=\frac{1}{\sqrt{2}}\left(\hat{\xi}_{j}(\vec{l})-i \hat{\pi}_{j}(\vec{l})\right), \\
& \mathbf{a}_{j}(\vec{l})=\frac{1}{\sqrt{2}}\left(\hat{\xi}_{j}(\vec{l})+i \hat{\pi}_{j}(\vec{l})\right),
\end{aligned}
$$

avec

$$
\begin{aligned}
& \hat{\xi}_{j}(\vec{l})=\alpha_{j} \mathbf{Q}_{j}(\vec{l}), \\
& \hat{\pi}_{j}(\vec{l})=\frac{1}{\alpha_{j} \hbar} \mathbf{P}_{j}(\vec{l}) .
\end{aligned}
$$

Les modes ainsi créés ne possèdent des amplitudes d'oscillation que dans une maille du réseau. Les variables cartésiennes des atomes $\mathbf{u}_{\alpha}(\kappa, \vec{l})$ pour ce mode sont projetées sur le vecteur propre $e_{j}(\alpha, \kappa)$. Ces excitations stationnaires ${ }^{78}$ et complètement localisées correspondent donc à un mode normal de la molécule décrit par le vecteur à $3 r$ composantes $e_{j}(\alpha, \kappa)$. En termes plus simples, les vibrations locales des molécules constituent des modes propres du système. Dans le monde réel, un mode a toujours une dispersion même si elle peut être très faible. Les états créés par la superposition 3.246 ne seront locaux que sur un temps comparable avec l'inverse de la largeur de bande $\Delta \omega_{j}$. Après il s'élargiront dans l'espace comme prévu pour un paquet d'onde quantique (voir section 2.1.4).

Pour une molécule non-linéaire, il existe au maximum $3 r-6$ modes internes. Ce sont ces modes qui sont présents quand la molécule se trouve isolée. Ils ont par définition le potentiel de créer des nappes de dispersion plates, pourvu la molécule reste rigide sous l'effet des forces intermoléculaires. Parmi les 6 modes restant dits externes, les trois modes acoustiques correspondent au déplacement du centre de gravité des molécules. Les nappes de dispersion leur correspondant sont forcément dispersives. Pour les trois autres, qui décrivent des librations (rotations de la molécule dans son intégralité autour du centre de gravité), le degré de dispersion dépendra du couplage entre les molécules. Pour des sphères idéales ce couplage serait strictement zéro. Ces points seront illustrés dans la section 3.16 ainsi que dans le chapitre de cet ouvrage qui traite de la dynamique des fullerènes.

Nous allons maintenant traiter le cas d'un cristal quelconque. La question à laquelle nous voudrions répondre peut se formuler ainsi: quelles conditions doivent être réunies pour permettre des excitations du réseau de manière locale? Dans ce contexte, le cas extrême que nous pouvons étudier est l'excitation d'un seul atome. La mécanique quantique nous répond tout de suite qu'une telle excitation ne serait stationnaire que si cet atome était complètement isolé du reste du réseau. C'est incompatible avec la condition que chaque atome doit posséder une position d'équilibre bien définie, ce qui ne peut être assurée que par l'intermédiaire d'interactions. Mais dès que des interactions sont présentes, l'oscillateur local ne constitue plus une fonction propre de l'Hamiltonien. Une excitation locale n'est donc pas stationnaire, c'est-à-dire la densité de probabilité de présence de la particule évoluera avec le temps.

Il est instructif de regarder ce problème sous un autre angle. Partons d'un état $\Psi(\alpha, \kappa, \vec{l})$, qui à $t=0$ décrit le déplacement d'un seul atome $(\kappa, \vec{l})$ dans une direction $\alpha$. Attention, dans ce cas cet atome ne peut pas se trouver dans un des états propres d'un oscillateur étant donné que, dû à la symétrie du puits de potentiel, $\langle\Psi|\mathbf{u}| \Psi\rangle=0$ pour toutes les fonctions propres d'un oscillateur. En s'appuyant sur le théorème d'Ehrenfest 3.125, l'évolution de la valeur moyenne $\left\langle\mathbf{u}_{\alpha}(\kappa, \vec{l})\right\rangle=\left\langle\Psi\left|\mathbf{u}_{\alpha}(\kappa, \vec{l})\right| \Psi\right\rangle$ est identique à l'évolution de la variable classique $u_{\alpha}(\kappa, \vec{l})$. Cette évolution peut être déduite facilement

\footnotetext{
78 Nous devons ici faire très attention à la terminologie. Il faut bien distinguer un état stationnaire, c'est à dire un état propre de l'Hamiltonien, d'une onde stationnaire.
} 

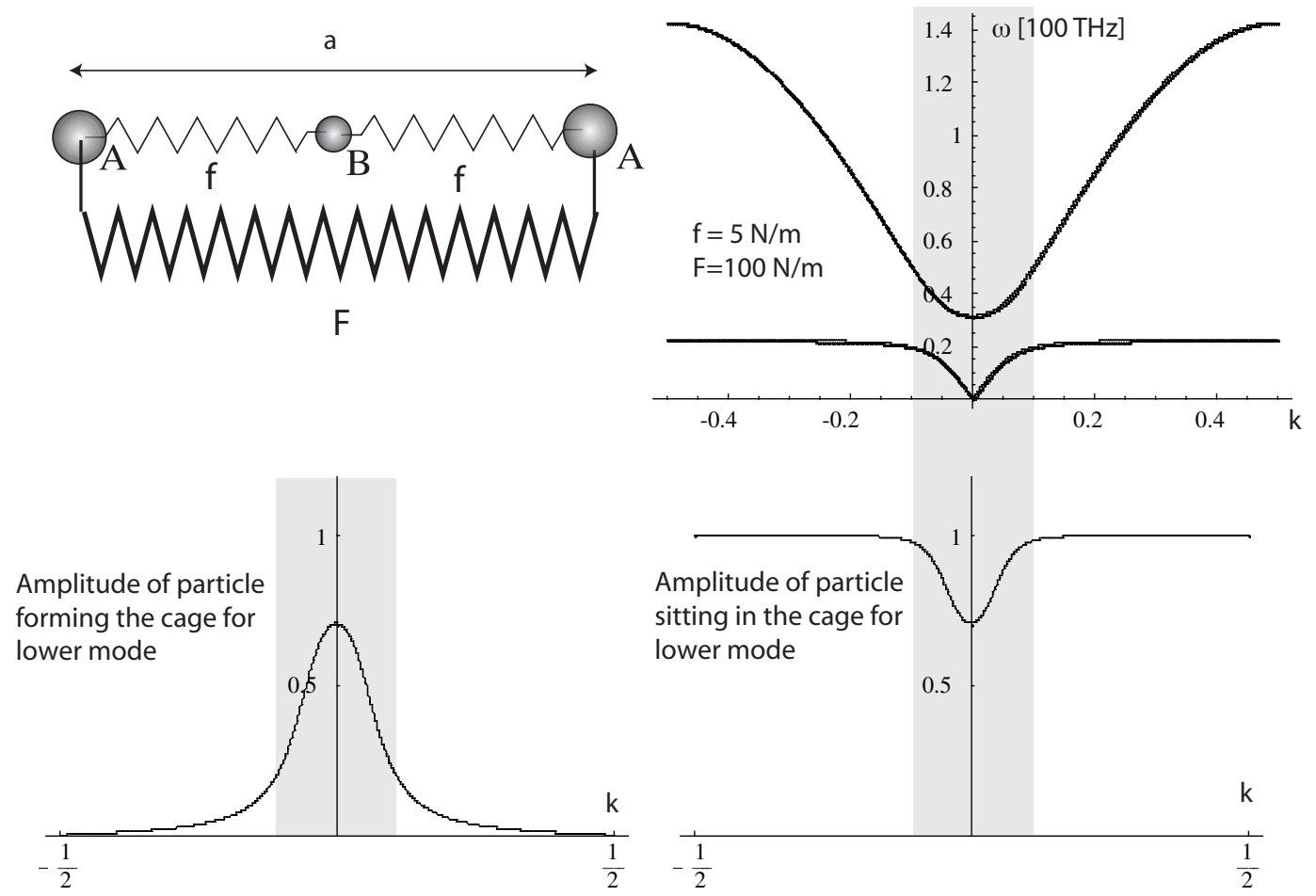

Figure 30. Chaîne linéaire à effet de cage (voir section 3.3.4) pour un choix relativement extrême du rapport entre les constantes de force $f$ et $F$. Les deux atomes n'oscillent ensemble que dans une petite région autour du centre de zone (parties à fond gris dans les diagrammes). En dehors de cette zone, l'atome qui se trouve dans la cage oscille seul pour les modes de la branche inférieure de la loi de dispersion. L'inverse est vrai pour la branche supérieure. Quand un atome oscille seul, les vecteurs propres peuvent être choisis reéls, exactement comme dans un système monoatomique. La branche inférieure est plate en dehors de la partie grise. Nous pouvons donc appliquer la superposition de l'expression 3.246 pour obtenir des modes stationnaires, qui seront en bonne approximation locaux. Une population de ces états à une température $T$ (le nombre d'occupation sera pareil pour tous, étant donné qu'ils partagent la même énergie) correspondra à des mouvements incohérents (sans relation de phase fixe) des atomes séquestrés. Ce raisonnement n'est pas applicable à la branche supérieure parce que celle-ci est très dispersive. Le fait que $f \ll F$ a la conséquence secondaire que les fréquences des modes auxquels les atomes séquestrés participent sont très petites. Le déplacement moyen de ces atomes sera donc très grand (voir expression 3.165). Ils risquent de montrer des effets anharmoniques plus prononcés que les cages.

de l'expression 3.213

$$
u_{\alpha}(\kappa, \vec{l} \mid t)=\frac{1}{\sqrt{m_{\kappa}}} \sum_{j=1}^{3 r} \sum_{\vec{k}}^{B Z} Q_{j}(\vec{k}) e_{j}(\alpha, \kappa \mid \vec{k}) e^{-i\left(\vec{k} \cdot \vec{l}-\omega_{j}(\vec{k}) t\right)} .
$$

Comme on peut le déduire facilement, cette superposition comporte des contributions de tous les modes auxquels l'ion en question participe, c'est-à-dire pour lequel l'élement $e_{j}(\alpha, \kappa \mid \vec{k})$ du vecteur propre n'est pas strictement nul. En général, les fréquences de tous ces modes diffèrent et le mouvement ainsi construit ne sera pas stationnaire. Assez rapidement l'amplitude de déplacement, c'est-à-dire l'impulsion, va être transférée aux atomes environnants. En peu de temps, l'état ne sera pratiquement plus discernable de l'état fondamental.

Si nous avons affaire à une surface de dispersion pratiquement plate, la situation est différente. C'est le cas quand le couplage est faible, créant comme on a vu dans le cas de la chaîne linéaire un effet de cage. La raison peut se trouver dans une grande différence de masse (découplage dynamique) ou de 
constantes de forces (découplage chimique). Dans le premier cas, les ions légers oscillent tellement plus vite que les ions plus lourds que ceux-ci ne peuvent pas suivre. Dans le deuxième cas des structures très rigides emprisonnent des ions faiblement liés. On peut considérer cette situation comme un cristal moléculaire inversé. Une énorme molécule rigide retient des atomes invités en otage. En fin de compte l'expression "faiblement liés" est à nuancer. L'ion prisonnier des cages est faiblement lié dans le sens où il peut faire des excursions de petite amplitude dans sa cellule. La cellule elle-même constitue une barrière forte lors d'éventuelles tentatives d'évasion.

Naturellement, des combinaisons des deux phénomènes (découplage dynamique et découplage chimique) sont possibles. La surface de dispersion étant plate, le déphasage entre les différents modes qui composent l'état local, va s'installer lentement. Néanmoins, l'impulsion devra se dissiper finalement ne serait ce qu'à cause de la présence des modes de basse fréquence acoustiques (i) auxquels tous les ions participent avec la même amplitude, (ii) dont la fréquence change avec le vecteur $\vec{k}$ et (iii) qui sont bel et bien présents dans la somme 3.249.

Par contre, quand nous nous limitons à effectuer la somme sur les modes plats d'une même fréquence nous créons toujours un motif de déplacement stationnaire, dont le degré de localisation dépendra du système. La molécule traitée ce dessus est un exemple illustrant cette affirmation. Un autre cas parlant est de nouveau l'atome dans une cage (voir figure 30).

\subsection{2 États cohérents}

Les phonons en tant qu'ondes stationnaires ne décrivent pas des déplacements dans le temps. Les densités de probabilité de présence des ions n'évoluent pas et restent donc, à cause de la symétrie des puits de potentiel, à tout instant centrées autour des positions d'équilibre des ions. Proprement peuplés, ces modes décrivent une situation incohérente en ce qui concerne les excitations, comme par exemple l'agitation des ions due au couplage avec un bain thermique. Une oscillation des probabilités de position des ions $\langle\mathbf{u}(\vec{l})\rangle$ requiert une superposition d'états pour plusieurs valeurs d'énergie. Pour un état macroscopique, comme une onde sonore, on demandera en plus que la détection d'un phonon composant cet état le laisse inchangé. Mathématiquement, cette condition est remplie si l'état est un état propre de l'opérateur d'annihilation

$$
\mathbf{a}_{j}(\vec{k})\left|\Psi_{\mathrm{coh}}(j, \vec{k})\right\rangle=\alpha\left|\Psi_{\mathrm{coh}}(j, \vec{k})\right\rangle .
$$

On peut vérifier que l'état

$$
\left|\Psi_{\mathrm{coh}}(j, \vec{k})\right\rangle=\exp \left(-\frac{|\alpha|^{2}}{2}\right) \sum_{n=0}^{\infty} \frac{\alpha^{n}}{n !}\left(\mathbf{a}_{j}^{+}(\vec{k})\right)^{n}|0\rangle
$$

remplit cette condition. Le nombre de phonons présents dans cet état -dit cohérent- satisfait à une distribution de Poisson

$$
P(n)=e^{-\langle n\rangle} \frac{\langle n\rangle^{n}}{n !}
$$

centrée autour de

$$
\left\langle n_{j}(\vec{k})\right\rangle=|\alpha|^{2},
$$

avec une largeur de

$$
\Delta n_{j}(\vec{k})=|\alpha|=\sqrt{\left\langle n_{j}(\vec{k})\right\rangle} .
$$

Les états cohérents ont été utilisés pour la première fois par Erwin Schrödinger pour expliquer le lien entre la mécanique quantique et la mécanique classique. Ils sont aussi appelés états classiques. Un état cohérent évolue dans le temps comme un état classique. La différence réside dans le fait que la probabilité de trouver un ion est étalée autour des trajectoires classiques. En augmentant le nombre de 
phonons, l'étalement devient de plus à plus imperceptible par rapport au déplacement. On s'approche alors d'une onde classique (voir la figure 29).

\subsection{L'analogie avec les électrons de Bloch}

Nous voudrions terminer cette discussion générale concernant les excitations dans un cristal, en confrontant le formalisme développé dans les chapitres précédant avec celui des électrons de Bloch. Les électrons dans un cristal interagissent comme nous avons vu en section 3.1 (équation 3.3) entre euxmêmes et avec les ions. C'est un problème beaucoup trop complexe pour un traitement quantitatif. Nous devons par conséquent développer des concepts qui nous permettent d'en enrayer la complexité. Comme dans le cas des phonons, nous chercherons à diagonaliser l'Hamiltonien, c'est-à-dire à découpler le système autant que possible. Une voie possible est de supposer que les électrons sont des particules indépendantes qui évoluent dans un potentiel périodique. Ce potentiel provient d'un côté des ions supposés au repos et de l'autre du champ moyen provenant des autres électrons.

Nous cherchons donc des solutions au problème

$$
\mathbf{H}|\psi\rangle=\left(-\frac{\hbar^{2}}{2 m} \vec{\nabla}^{2}+U(\vec{r})\right)|\psi\rangle=E_{1}|\psi\rangle
$$

avec

$$
U(\vec{r})=U(\vec{r}+\vec{l}), \quad \vec{l}=n_{1} \vec{a}_{1}+n_{2} \vec{a}_{2}+n_{3} \vec{a}_{3} .
$$

Etant donné que l'Hamiltonien possède la symétrie de translation du réseau nous pouvons invoquer les mêmes théorèmes de la théorie des groupes qui nous ont permis de caractériser les excitations de réseau. En clair, nous pouvons choisir les fonctions d'onde de façon à ce qu'elles remplissent la condition

$$
\psi(\vec{r}+\vec{l})=e^{i \vec{k} \cdot \vec{l}} \psi(\vec{r})
$$

pour $\vec{k} \in \mathrm{BZ}$. Jusqu'ici, c'est le même résultat que la relation 3.198 pour les ions. Mais nous pouvons aller immédiatement ${ }^{79}$ plus loin en exploitant le fait que $\psi(\vec{r})$ est une fonction à une particule et donc à une seule variable. Il suit que cette fonction diffère d'une maille à l'autre seulement d'un facteur de phase. Elle doit donc pouvoir s'écrire comme

$$
\psi(\vec{r} \mid \vec{k})=e^{i \vec{k} \cdot \vec{r}} u(\vec{r} \mid \vec{k})
$$

avec la fonction périodique

$$
u(\vec{r}+\vec{l} \mid \vec{k})=u(\vec{r} \mid \vec{k}), \quad \vec{l}=n_{1} \vec{a}_{1}+n_{2} \vec{a}_{2}+n_{3} \vec{a}_{3} .
$$

En remplaçant cette expression dans l'Hamiltonien, nous obtenons comme équation définissant les fonctions $u(\vec{r} \mid \vec{k})$

$$
E_{1}(\vec{k}) u(\vec{r} \mid \vec{k})=\left(\frac{\hbar^{2}}{2 m}\left(\frac{1}{i} \vec{\nabla}+\vec{k}\right)^{2}+U(\vec{r})\right) u(\vec{r} \mid \vec{k}) .
$$

La periodicité de la fonction $u$ restreint le problème à trouver les valeurs propres de cette matrice hermitienne à une seule maille primitive du cristal. Puisque le volume de cette maille est fini et fixé nous nous attendons pour chaque vecteur $\vec{k}$ à un nombre de solutions infini mais dénombrable. Comme dans le cas des électrons libres avec conditions aux limites périodiques, les énergies vont être discrètes pour chaque vecteur d'onde $\vec{k}$. Nous étiquetterons ces énergies par l'indice $n$. Le vecteur d'onde $\vec{k}$ ne figure que comme paramètre dans l'equation 3.260. L'énergie $E_{n}(\vec{k})$ va par conséquent changer continûment

\footnotetext{
${ }^{79}$ Dans le cas des phonons nous n'avons pas vraiment déduit l'Ansatz 3.200 de l'expression 3.198.
} 


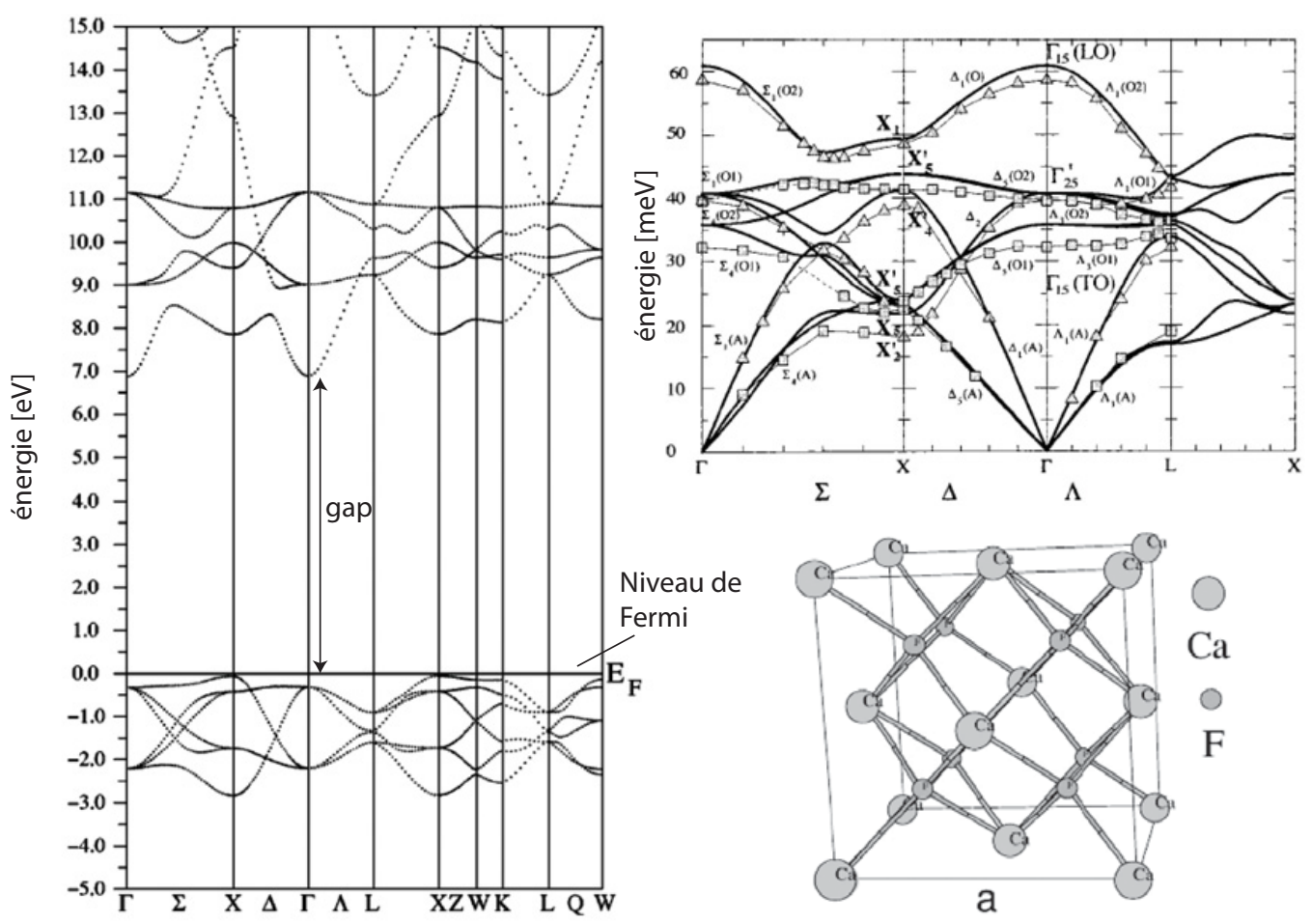

Figure 31. Comparaison de la structure de bande électronique avec celle des courbes de phonons. Les résultats sont obtenus par des calculs ab intio pour le conducteur superionique $\mathrm{CaF}_{2}$ [35]. Dans les deux cas, la symétrie de translation amène à des relations de dispersion périodiques dans l'espace réciproque. Les excitations sont discrètes pour une valeur du vecteur d'onde $\vec{k}$ donnée. Le vecteur d'onde figure comme paramètre dans l'Hamiltonien. Les courbes de dispersion sont donc quasi-continues. La différence majeure réside dans le fait que le nombre de bande est infini, là où on dénombre exactement $3 r$ branches pour les phonons. Nous voudrions aussi attirer l'attention sur la grande différence d'échelle d'énergie entre les électrons et les phonons. L'énergie maximale des phonons $(\approx 60 \mathrm{meV})$ est beaucoup plus faible que le gap entre états électroniques occupés et vides de plusieurs $\mathrm{eV}$. L'approximation adiabatique (voir section 3.1) est par conséquent pleinement justifiée.

avec $\vec{k}$. Nous obtenons comme résultat final une description des électrons indépendants dans un potentiel périodique en termes de familles de fonctions de bandes continues.

Le calcul des bandes électroniques ressemblent beaucoup au calcul des surfaces de dispersion des phonons. La raison est simple. Nous nous appuyons dans les deux cas sur la symétrie. Le fait que l'électron est une particule dans un potentiel extérieur là où les ions sont un ensemble de particules couplées de manière harmonique, a néanmoins des conséquences importantes. La différence la plus évidente est le nombre de branches. Pour les ions il y a $3 r$ courbes de dispersion, tandis qu'il y a une infinité de bandes pour les électrons de Bloch. Les niveaux de ces bandes son remplis un par un (ou deux par deux si on inclut le spin) jusqu'au niveau de Fermi. Les phonons se comportent par contre comme des particules de Bose permettant un remplissage multiple des oscillateurs. Nous donnons un exemple concret dans la figure 31 .

\subsection{Calculs des éléments de la matrice dynamique}

Nous avons formellement résolu le problème de la dynamique de réseau en diagonalisant l'Hamiltonien à l'aide des modes normaux. Cette percée théorique nous permet de formuler des constats assez généraux 
concernant la thermodynamique et la réponse linéaire d'un système harmonique. Dans la pratique, il faut néanmoins connaître les constantes de force pour calculer les spectres des phonons. Ce n'est pas une tâche triviale pour des systèmes complexes. Deux approches sont possibles. Soit on modélise l'interaction entre les ions par des potentiels empiriques appellés champs de force, soit on détermine directement le changement de l'énergie du système en fonction des déplacements ioniques avec la théorie de la fonctionnelle de la densité (Density Functional Theory) [25] (voir chapitre de M. Johnson et co-auteurs dans ce livre). Nous nous contentons ici de quelques remarques sommaires.

Chaque paire d'ions contribue pour un bloc de dimension $(3 \times 3)$ à la matrice dynamique.

$$
\Phi\left(\kappa, \vec{l}, \kappa^{\prime}, \vec{l}^{\prime}\right)=\left(\begin{array}{lll}
a & b & c \\
d & e & f \\
g & h & i
\end{array}\right)
$$

Sans information supplémentaire et en l'absence de symétrie particulière, il faut donc neuf paramètres pour le spécifier. C'est une situation vite insatisfaisante quand le nombre d'ions augmente. Dans beaucoup de cas, on peut supposer que le potentiel entre deux ions ne dépend que de la distance qui les séparent

$$
\Phi\left(\vec{r}_{1,1} \ldots \vec{r}_{N, r}\right)=\frac{1}{2} \sum_{\vec{l}, l^{\prime}, \kappa, \kappa^{\prime}} \Phi\left(\left|\vec{r}_{l, \kappa}-\vec{r}_{l^{\prime}, \kappa^{\prime}}\right|\right) .
$$

Cette restriction a pour conséquence que les blocs des constantes de forces doivent satisfaire

$$
\Phi_{\alpha \beta}\left(\begin{array}{ll}
\vec{l} & \vec{l}^{\prime} \\
\kappa & \kappa^{\prime}
\end{array}\right)=(\mathrm{T}-\mathrm{L}) \frac{\mathrm{r}_{\alpha} \mathrm{r}_{\beta}}{\mathrm{r}^{2}}-\mathrm{T} \delta_{\alpha \beta}
$$

avec la constante de force longitudinale définie par

$$
\mathrm{L}=\left.\frac{\mathrm{d}^{2} \Phi\left(\left|\vec{r}_{l, \kappa}-\vec{r}_{l^{\prime}, \kappa^{\prime}}\right|\right)}{\mathrm{dr}^{2}}\right|_{\mathrm{r}=\left|\vec{R}_{l^{\prime} \kappa^{\prime}}-\vec{R}_{l \kappa}\right|}
$$

ainsi que la constante de force transverse définie par

$$
\mathrm{T}=\left.\frac{1}{\mathrm{r}} \frac{\mathrm{d} \Phi\left(\left|\vec{r}_{l, \kappa}-\vec{r}_{l^{\prime}, \kappa^{\prime}}\right|\right)}{\mathrm{dr}}\right|_{\mathrm{r}=\left|\vec{R}_{l^{\prime} \kappa^{\prime}}-\vec{R}_{l \kappa}\right|} .
$$

L'analogie mécanique de ces deux constantes de force sont les ressorts toroïdaux et les ressorts trapézoïdaux, respectivement. La constante longitudinale $L$ est sollicitée seulement si le déplacement a une composante le long de la ligne de séparation. La constante transversale $T$ assure seule les forces de rappel dans le cas où les déplacements sont perpendiculaires à la ligne de séparation. Un cas où les constantes de forces peuvent être calculées explicitement est le potentiel de Coulomb. Dans ce cas

$$
\begin{aligned}
& \Phi_{\alpha \beta}\left(\begin{array}{cc}
\vec{l} & \vec{l}^{\prime} \\
\kappa & \kappa^{\prime}
\end{array}\right)=-\mathrm{Z}_{\kappa} \mathrm{Z}_{\kappa^{\prime}} e^{2} \lim _{\vec{r} \rightarrow 0}\left\{\frac{\partial^{2}}{\partial \mathrm{r}_{\alpha} \partial \mathrm{r}_{\beta}} \frac{1}{\mid \vec{r}-\left[\vec{R}\left(l^{\prime}, \kappa^{\prime}\right)-\vec{R}(0, \kappa] \mid\right.}\right\} \\
= & \mathrm{Z}_{\kappa} \mathrm{Z}_{\kappa^{\prime}} e^{2}\left\{\frac{\text { delta } a_{\alpha \beta}}{\mathrm{r}^{3}}-\frac{3 \mathrm{r}_{\alpha} \mathrm{r}_{\beta}}{\mathrm{r}^{5}}\right\}_{\vec{r}=\left|\vec{R}\left(l^{\prime}, \kappa^{\prime}\right)-\vec{R}(0, \kappa)\right|}, \quad l^{\prime} \neq 0 \quad \text { et } \quad \kappa \neq \kappa^{\prime} .
\end{aligned}
$$

On identifie en comparant avec l'équation 3.263

$$
L=-2 T, \quad \text { et } \quad T=-\frac{\mathrm{Z}_{\kappa} \mathrm{Z}_{\kappa^{\prime}} e^{2}}{r^{3}} .
$$

Pour avoir une idée de la valeur absolue de ces constantes de forces, nous donnons l'expression

$$
T[\mathrm{~N} / \mathrm{m}]=-230.685 \frac{\mathrm{Z}_{\kappa} \mathrm{Z}_{\kappa^{\prime}}}{r^{3}\left[\AA^{3}\right]} .
$$



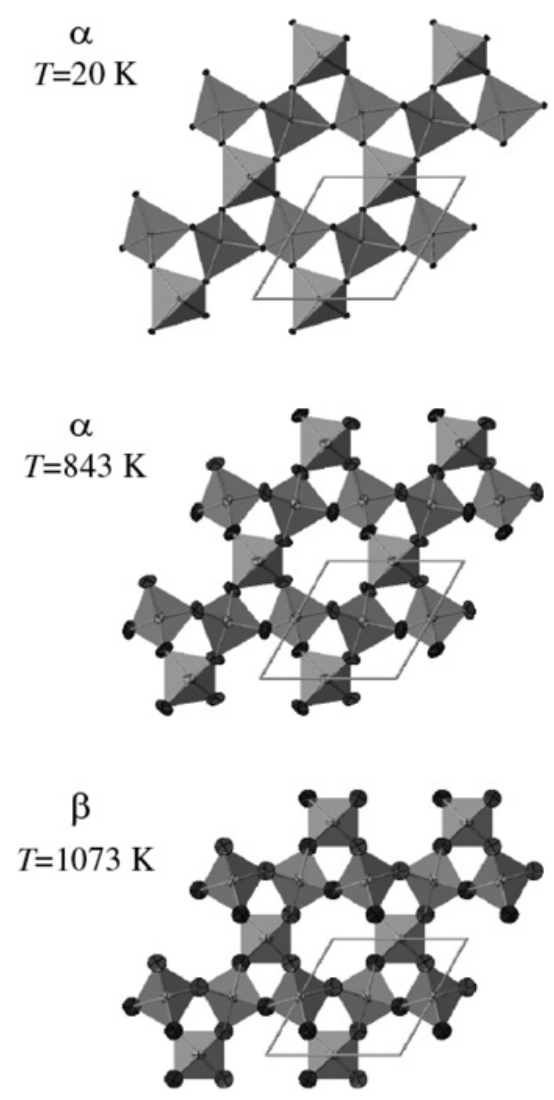

Figure 32. Structure des deux phases du quartz selon [30]. A haute température, les tétraêdres de $\mathrm{SiO}_{4}$ sont reliés par un axe de trans-rotation d'ordre 6 . A basse température, il ne reste qu'un axe d'ordre 3 suite aux mouvements de tétraèdres $\mathrm{SiO}_{4}$ presque rigides. L'augmentation des fluctuations des atomes avec la température est montrée schématiquement par la taille des ellipses, qui représentent les positions atomiques.

Deux charges élémentaires distantes d'un Å produisent des constantes de force longitudinales de $L=460[\mathrm{~N} / \mathrm{m}]$. Cette valeur numérique permet de situer les constantes de forces rencontrées dans les solides.

\subsection{Exemple concret : les phonons du quartz}

Pour faire le lien entre la théorie de la dynamique de réseau et le monde des matériaux, nous présentons deux exemples concrets. Le premier exemple traite le cas du quartz, qui est l'archétype de la classe des minéraux. Sa structure est à la fois suffisamment symétrique et suffisamment complexe pour montrer la quasi-totalité des aspects de la dynamique de réseau traitée dans la partie théorique. La partie manquante relève de la présence d'unités moléculaires. Elle va faire l'objet de notre attention dans le deuxième exemple qui traitera de la dynamique du réseau des fullèrenes (voir aussi le chapitre de J. Cambedouzou de cet ouvrage dédié entièrement à ce sujet).

Le $\mathrm{SiO}_{2}$ cristallin possède une multitude de phases de basse et de haute pression. Toutes ces phases se construisent à partir de tétraèdres $\mathrm{SiO}_{4}$ plus ou moins réguliers. Ils forment des réseaux dits de charpente, en partageant des atomes d'oxygène à leurs sommets (voir figure 32). Une phase particulièrement bien connue est le quartz. Le quartz existe dans une phase basse température $\alpha$ ainsi que dans une phase haute température $\beta$. La maille primitive du quartz possède une symétrie hexagonale. Le réseau est décrit par 


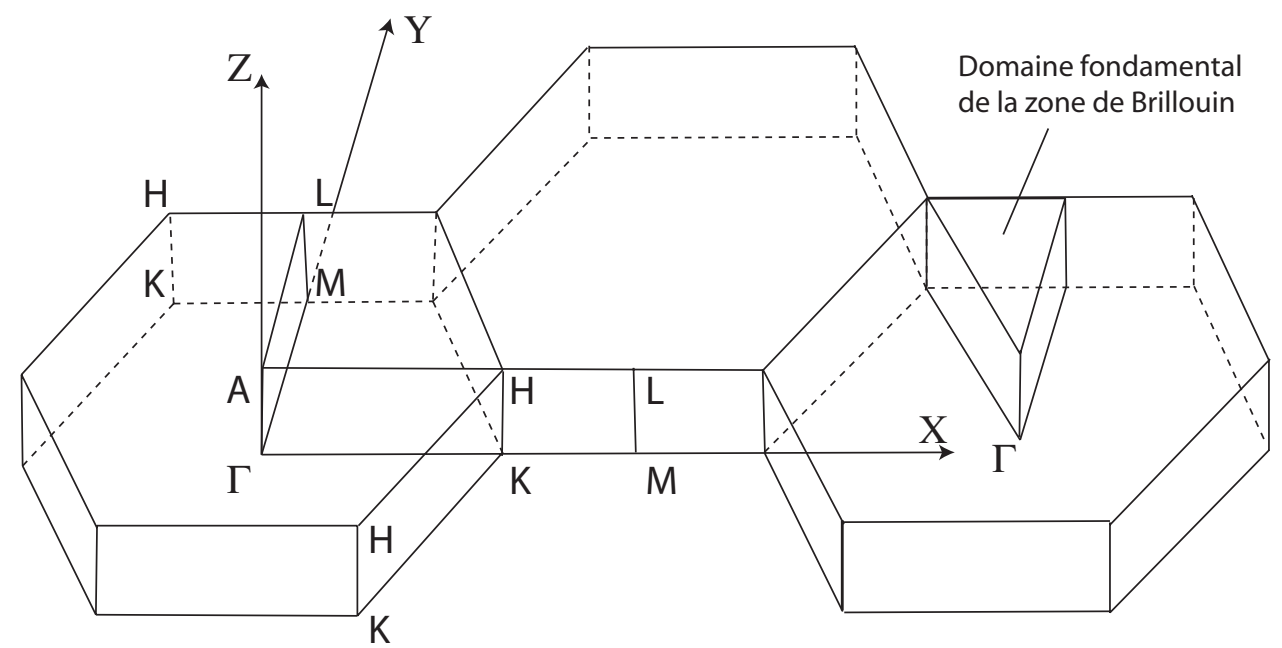

Figure 33. Zones de Brillouin du quartz. Les points de haute symétrie sont marqués par des symboles. On ne montre que la moitié haute des zones qui forment un réseau hexagonal. Toute l'information concernant les excitations est contenue dans la partie irréductible aussi appelée domaine fondamental de la première zone de Brillouin. Le volume fondamental n'est que $1 / 24$ du volume total de la zone de Brillouin.

des vecteurs primitifs, qui en coordonnées cartésiennes $\left(\vec{e}_{i}, i=1,2,3\right)$ sont donnés par

$$
\vec{a}=a \vec{e}_{1}, \quad \vec{b}=-\frac{1}{2} a \vec{e}_{1}+\frac{\sqrt{3}}{2} a \vec{e}_{2}, \quad \vec{c}=c \vec{e}_{3} .
$$

avec les constantes de mailles $a \approx 4.91 \AA$ et $c \approx 5.41 \AA$.

Le réseau réciproque d'un réseau hexagonal est de nouveau hexagonal. Les vecteurs définissant les zones de Brillouin sont

$$
\vec{a}^{*}=\frac{2 \pi}{a}\left(\vec{e}_{1}+\frac{1}{\sqrt{3}} \vec{e}_{2}\right), \quad \vec{b}^{*}=\frac{4 \pi}{a \sqrt{3}} \vec{e}_{2}, \quad \vec{b}^{*}=\frac{2 \pi}{c} \vec{e}_{3} .
$$

Le motif de la maille primitive du quartz est composé de trois unités chimiques de $\mathrm{SiO}_{2}$. Ces 9 atomes dans la maille primitive nous amènent à 27 degrés de liberté et donc à 27 surfaces de dispersion.

Le $\alpha$-quartz possède deux axes de symétrie. Un axe de trans-rotation ternaire dans la direction $\vec{c}$ et un axe de rotation binaire dans la direction $\vec{a}$. Si on veut se donner une image simple de la structure du quartz on peut s'imaginer les atomes disposés sur un escalier en hélice avec un pas de $1 / 3 c$. On monte ou on descend d'un atome de silicium à un autre en tournant de $\pm 2 \pi / 3$ autour de l'axe $\vec{c}$ et en progressant simultanément de $1 / 3 \vec{c} .{ }^{80}$ Quand on passe de la phase $\alpha$ à la phase $\beta$, l'axe de symétrie d'ordre 3 devient un axe d'ordre 6 .

Les positions de tous les atomes dans la maille primitive de l' $\alpha$-quartz peuvent se générer avec l'aide des opérations de symétrie à partir de 6 paramètres structuraux. En plus des constantes de maille, il faut connaître la position d'un silicium représentatif et d'un oxygène représentatif. Ces positions sont définies par

$$
\vec{r}_{\mathrm{Si}}=u \vec{a} \quad \vec{r}_{\mathrm{O}}=x \vec{a}+y \vec{b}-z \vec{c}
$$

\footnotetext{
${ }^{80}$ En fonction de la chiralité de l'hélice on doit en principe distinguer deux formes énantiomères de chaque phase du quartz. Etant donné que la chiralité n'influe pas sur les surfaces de dispersions, nous ne prêterons pas attention à cette complication supplémentaire.
} 

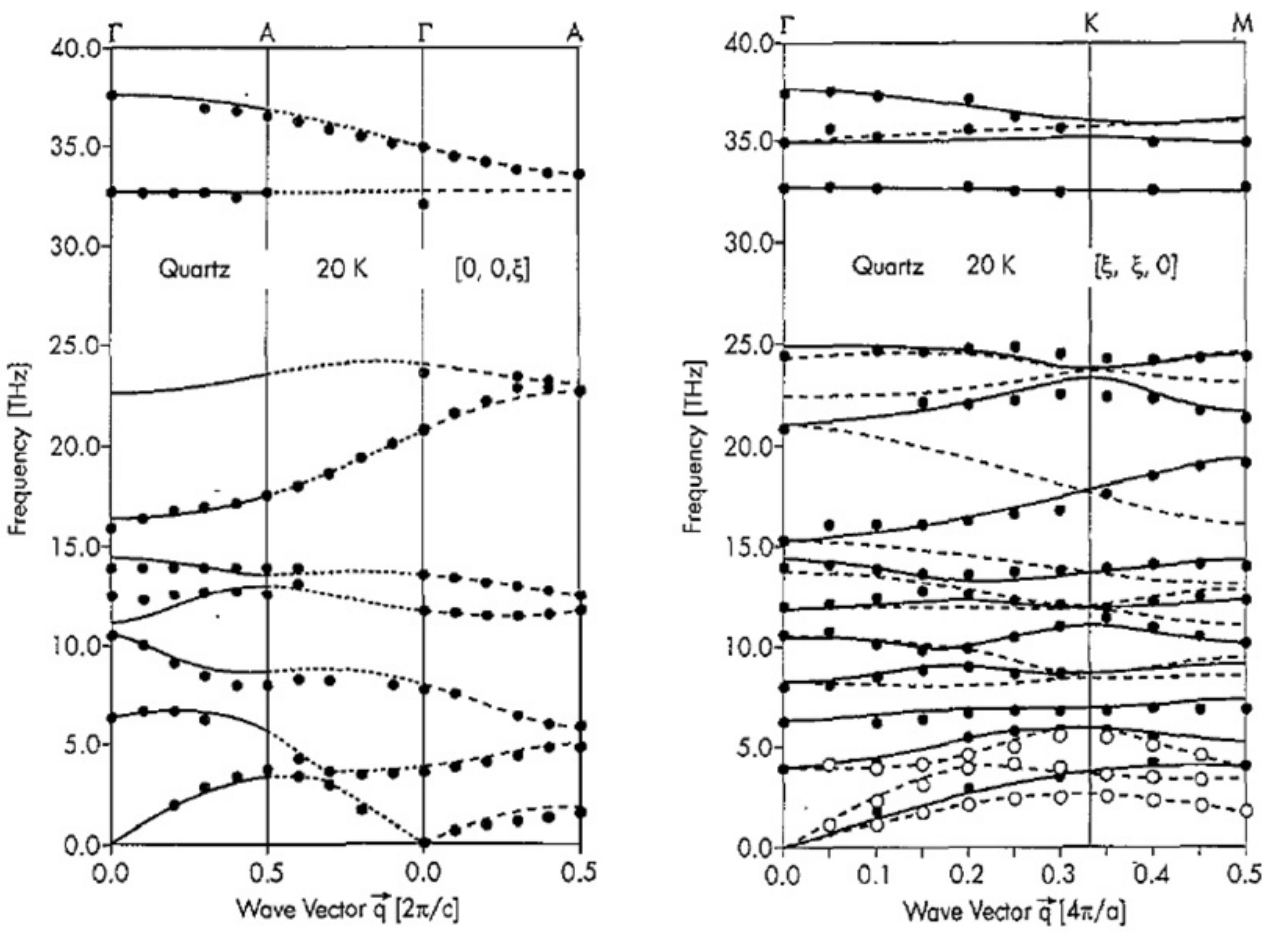

Figure 34. Courbes de dispersion du quartz $\alpha$ pour les deux directions de haute symétrie. Les cercles correspondent aux données expérimentales obtenues par diffusion des neutrons [26]. Les lignes correspondent aux résultats des calculs de dynamique de réseau [27]. Dans la direction $\vec{z}$ on a 3 représentations irréductibles unidimensionnelles appelées $\Delta_{1}, \Delta_{2}$ et $\Delta_{3}$. Parmi les modes de chaque représentation figure un mode acoustique. On constate que la dispersion (dépendance forte de la fréquence en fonction du vecteur d'onde) de ces modes acoustiques pénètre assez profondément dans la région des modes optiques. Par ailleurs, il y a des modes extrêmement plats autour de $32 \mathrm{THz}$. $\Delta_{2}$ et $\Delta_{3}$ sont dégénérées en centre de zone (point $\Gamma$ ) et $\Delta_{1}$ et $\Delta_{2}$ en bord de zone (point A). Ces dégénérescences imposées par la symétrie nous permettent de déplier les courbes de dispersion, c'est-à-dire que nous pouvons les représenter comme s'il s'agissait des courbes de dispersion continues d'un cristal, qui possède une constante de maille trois fois plus petite, et par conséquent une zone de Brillouin trois fois plus étendue le long de l'axe $\vec{c}$. Déplier la zone de Brillouin le long de l'axe $\vec{c}$ n'est pas un pur exercice de représentation. Si on mesure ces courbes avec les neutrons (voir le chapitre II) on observera une vraie continuité de l'intensité entre les courbes qui sont jointes au centre et en bord de zone. Si on effectue les mesures le long de l'axe hexagonal de l'espace réciproque alors on n'observera qu'au maximum neuf branches simultanément, qui appartiendront toutes à la même représentation irréductible. De plus, en passant d'une zone à l'autre on changera de représentation. Le spectre des excitations se décompose clairement en deux parties séparées par un gap entre 25 et $32 \mathrm{THz}$. Dans la partie haute, ce sont les liaisons covalentes entre silicium et oxygène qui sont sollicitées, induisant une forte distorsion des tétraèdres. A plus basse fréquence, les tétraèdres se comportent de plus en plus comme des unités rigides. Les courbes pour différentes représentations se croisent sans difficulté. Cela se voit particulièrement bien dans la direction $\Gamma$-K-M. Il existe plusieurs points $(\vec{q}, v)$ pour lesquels deux courbes appartenant à la même représentation arrivent presque à se toucher. L'anti-croisement mentionné en section 3.10.4 (voir figure 28) est donc très faible dans ces cas.

avec les valeurs

$$
x \approx 0.414, \quad y \approx 0.266, \quad z \approx 0.119, \quad u \approx 0.470
$$

Le quartz $\beta$ possède une symétrie plus élevée, ce qui réduit le nombre des paramètres libres à 2 :

$$
x, \quad y=x / 2, \quad z=1 / 6, \quad u=1 / 2 .
$$




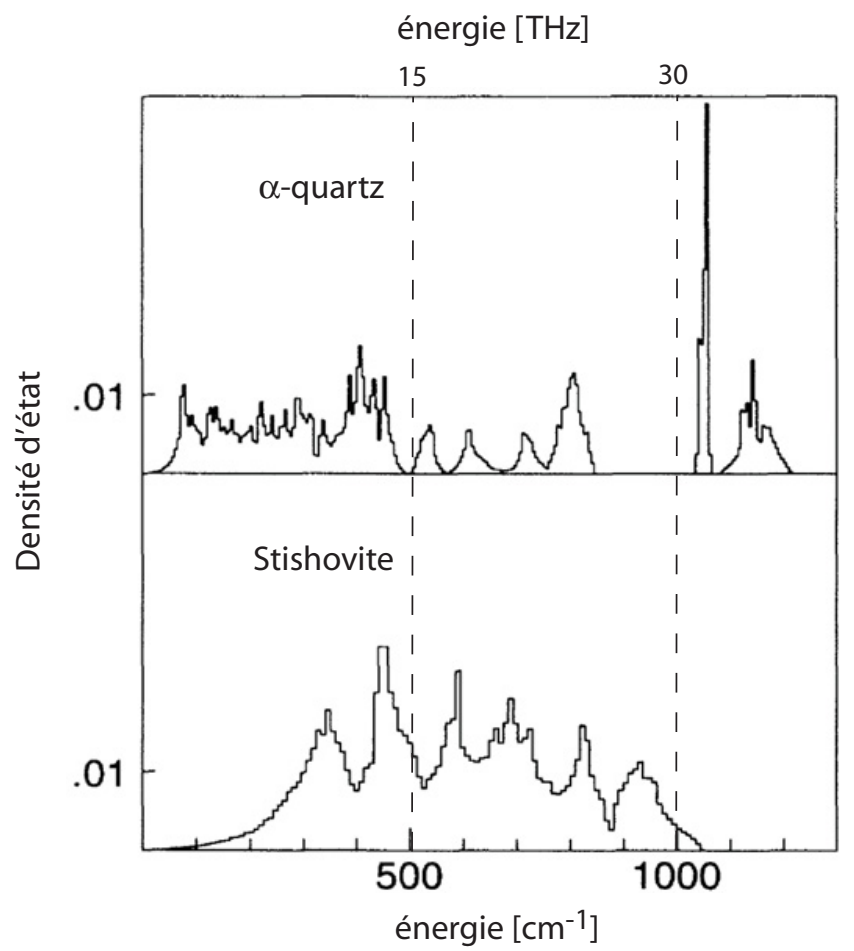

Figure 35. Densité d'états du quartz $\alpha$ et comparaison avec celle de la stishovite, qui est un autre polymorphe tétragonal de la silice $\mathrm{SiO}_{2}$. Les résultats sont obtenus à partir d'un calcul ab initio [29]. Ces densités d'états constituent la base du calcul des fonctions thermodynamiques que nous montrons en figure 36 .

La symétrie du quartz ${ }^{81}$ ne comportant que très peu d'éléments, il n'existe que deux directions dans l'espace réciproque pour lesquelles le groupe de symétrie du vecteur $\vec{k}$ n'est pas trivial. Il s'agit en coordonnées cartésiennes des directions $\vec{z}$ et $\vec{x}$ (voir figure 33 ).

Dans la direction $\vec{z}$, le groupe de symétrie est composé des opérations d'hélice. La matrice dynamique se décompose en 3 blocs de dimension 9. Les courbes de dispersion correspondant à ces représentations irréductibles unidimensionnelles sont appelées $\Delta_{1}, \Delta_{2}$ et $\Delta_{3}$. Dans la direction $\vec{x}$, il n'existe que 2 représentations unidimensionnelles irréductibles de multiplicité 13 et 14. Elles portent les noms $\mathrm{T}_{1}$ et $\mathrm{T}_{2}$ et elles sont, par rapport à l'axe binaire, respectivement symétrique et anti-symétrique.

Nous montrons les courbes de dispersion sur la figure 34 pour les deux directions de haute symétrie. Les résultats expérimentaux sont confrontés à des calculs de dynamique du réseau. On obtient un accord tout à fait correct pour un système d'une telle complexité. Les courbes de dispersion du quartz peuvent être décrites également de manière très satisfaisante par des calculs de DFT [28]. Il est instructif de regarder l'ordre de grandeur des constantes de force. La constante longitudinale effective (prenant en compte le potentiel de courte portée aussi bien que le potentiel de Coulomb) vaut environ 500 N/m pour l'interaction entre silicium et oxygène voisins (distants d'à peu près $1.6 \AA$ ). Pour les paires d'oxygène d'un tétraèdre (distantes d'à peu près $2.6 \AA$ ) nous obtenons $L \approx 50[\mathrm{~N} / \mathrm{m}]$. Ceci reflète très bien le caractère très covalent des liaisons $\mathrm{Si}-\mathrm{O}$. C'est un indicateur montrant que des forces autres que de paires jouent un rôle important dans la dynamique du quartz. En particulier, l'angle O-Si-O définissant les tétraèdres, résiste fort à une distorsion. C'est cette propriété qui identifie les tétraèdres comme des

81 Quartz voudra dire dorénavant quartz $\alpha$, à moins que l'on ne spécifie explicitement autre chose. 
Energie interne

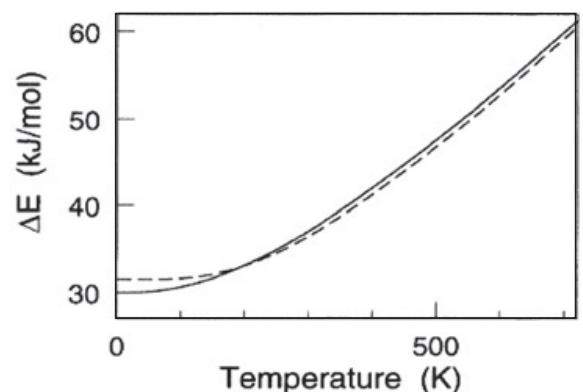

Energie libre

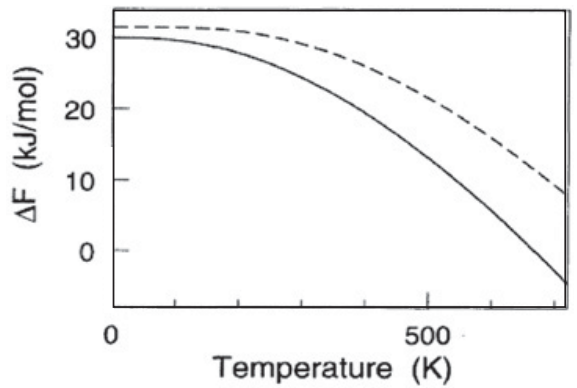

Entropie

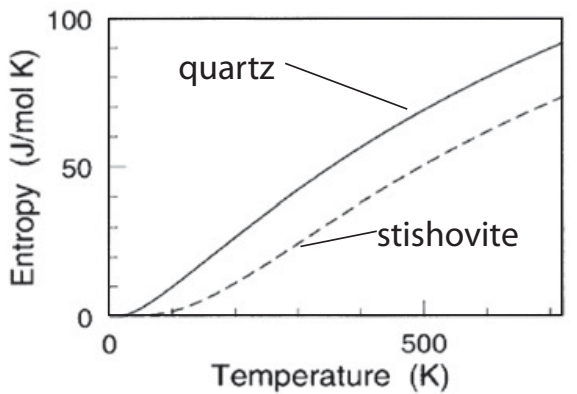

Chaleur spécifique

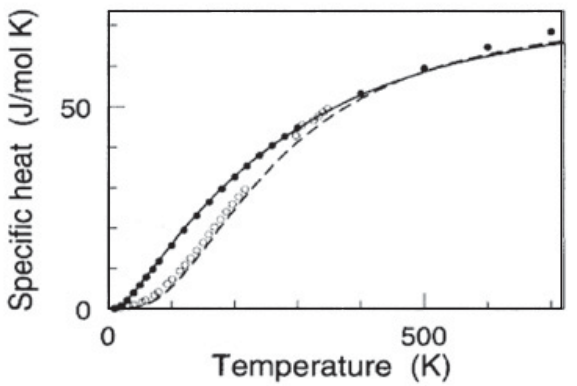

Figure 36. Fonctions thermodynamiques pour le quartz et pour la stishovite, obtenues avec les formules de la section 3.8 à partir des densités d'états de la figure 35 [29]. L'énergie interne est assez plate à basse température. Elle augmente à partir du moment où l'occupation thermique des niveaux prend le dessus sur les fluctuations quantiques. La contribution des fluctuations quantiques est moins importante dans le quartz que dans la stishovite parce que le spectre intégré du quartz est plus mou. L'entropie du quartz est toujours supérieure à celle de la stishovite. Ceci s'explique par la présence de modes de très basses fréquence. Ces modes sont également responsables de l'excès de chaleur spécifique du quartz par rapport à celle de la stishovite. On présente aussi des valeurs expérimentales pour la chaleur spécifique du quartz. L'écart par rapport aux courbes calculées s'explique par les contributions anharmoniques. Il est intéressant de constater que l'énergie libre du quartz passe en-dessous de zéro à des températures supérieures à $700 \mathrm{~K}$. Les calculs purement harmoniques prédisent donc déjà une instabilité structurale, qui n'est rien d'autre que la transition de phase entre le quartz $\alpha$ et le quartz $\beta$ observée expérimentalement.

unités assez rigides. Ces forces à trois corps (ou l'angle intervient) sont trop complexes pour être discutées ici. Nous voulons juste mentionner qu'elles sont décrites dans l'exemple numérique donné plus haut de manière effective, c'est-à-dire par des forces entre les atomes d'oxygène d'un tétraèdre. Une complication supplémentaire propre à tous les oxydes, et dont nous ne parlerons pas non plus, est que l'ion oxygène $O^{2-}$ est hautement polarisable, nécessitant l'utilisation d'un modèle en "couches" (shell model), si on veut obtenir des paramètres physiques [27].

Une fois que l'on connait le spectre des phonons, on peut calculer les fonctions thermodynamiques à partir des relations énoncées dans la section 3.8. Nous présentons quelques résultats pour le quartz en figure 36 .

\subsection{Exemple concret : la dynamique des fullerènes $\mathrm{C}_{60}$}

Le quartz est un cristal dont les unités de base sont les atomes. Même s'il est tout à fait vrai que ces atomes forment des unités structurales secondaires assez rigides (les tétraèdres $\mathrm{SiO}_{4}$ ), il n'y a pas vraiment de séparation des échelles d'énergie entre modes externes (translation et rotation des 


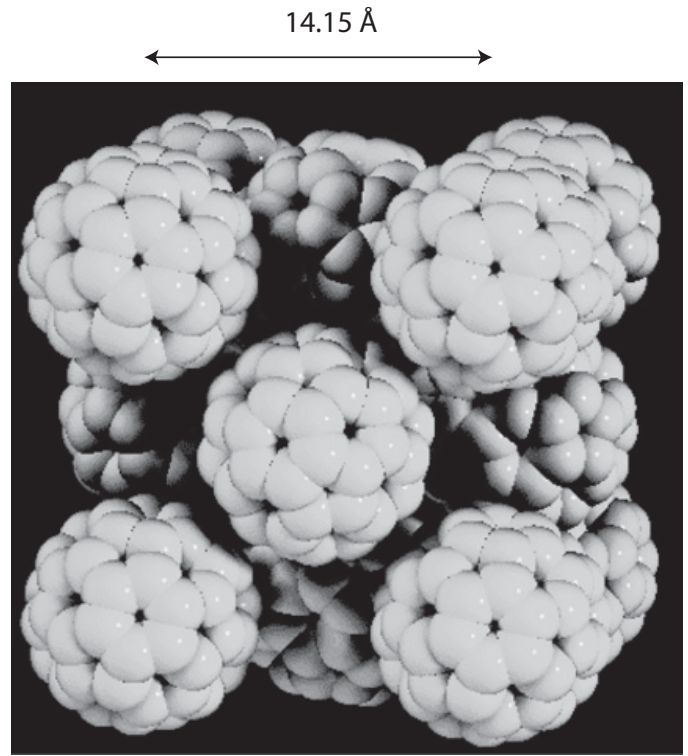

$9.9 \AA$

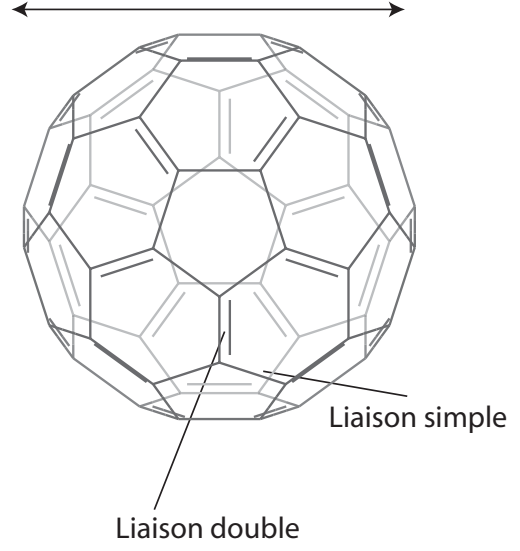

Figure 37. Structure du $\mathrm{C}_{60}$ pur dans sa phase monomère (Pa3). La molécule $\mathrm{C}_{60}$ possède deux types de liaison. Les liaisons plus courtes dites doubles sont responsables de la facilité avec laquelle le $\mathrm{C}_{60}$ polymérise.

tétraèdres) et internes (déformation des tétraèdres). Cette dimension supplémentaire nécessite en général la présence de molécules. Ces cristaux moléculaires nous ouvrent au passage, une porte vers la chimie.

Les cristaux de fullerènes -et en particulier ceux du $\mathrm{C}_{60}$ - constituent un exemple idéal de cristal moléculaire. La symétrie extrêmement élevée de la molécule et le fait qu'elle soit composée d'une seule espèce atomique produisent une panoplie d'effets dynamiques. Ces effets sont clairement discernables dans les résultats bruts, soit de la théorie soit des expériences. Ils sont donc d'une grande valeur pédagogique.

Il y a une vingtaine d'années, on a observé expérimentalement, pour la première fois, des molécules entièrement constituées de carbone et complètement fermées (fullerènes). Cette découverte a été récompensée par le prix Nobel de chimie en 1996. Quelques années après la première identification de fullerènes isolés, on a découvert un procédé de fabrication qui fournissait des quantités macroscopiques de $\mathrm{C}_{60}$ et de $\mathrm{C}_{70}$, les deux membres les plus stables de la famille des fullerènes. Ceci a ouvert la voie à l'étude de leurs propriétés à l'état solide.

A haute température, les fullerènes forment des structures cristallines où les molécules sont en rotation presque libre (phase rotationnelle), avant de s'évaporer dans la phase gazeuse. Dans le cadre de la chimie conventionnelle, les molécules neutres de fullérène devraient avoir très peu tendance à former des liaisons covalentes entre elles, étant donné que toutes leurs liaisons sont en résonance entre elles (molécules aromatiques). Mais on peut tirer la conclusion inverse si on représente la cage comme un ensemble alterné de simples et doubles liaisons. En réalité, les fullerènes se situent entre ces deux extrêmes. Par conséquent, les molécules de fullerènes ont une forte tendance à donner lieu à des réactions chimiques, pourvu qu'elles soient catalysées dans des conditions convenables, telles que sous irradiation de la lumière laser, par l'application de fortes pressions à température élevée ou par un dopage avec une quantité convenable d'ions alcalins (par exemple $\mathrm{AC}_{60}, \mathrm{~A}=\mathrm{K}, \mathrm{Rb}, \mathrm{Cs}$ ou $\mathrm{Na}_{4} \mathrm{C}_{60}$ ). Il se forme alors une grande variété de topologies de polymères (voir figure 38 pour deux exemples concrets). Les systèmes $\mathrm{AC}_{60}$ et $\mathrm{Na}_{4} \mathrm{C}_{60}$ sont particulièrement intéressants car leur polymérisation est réversible et peut être étudiée en fonction de la température. 

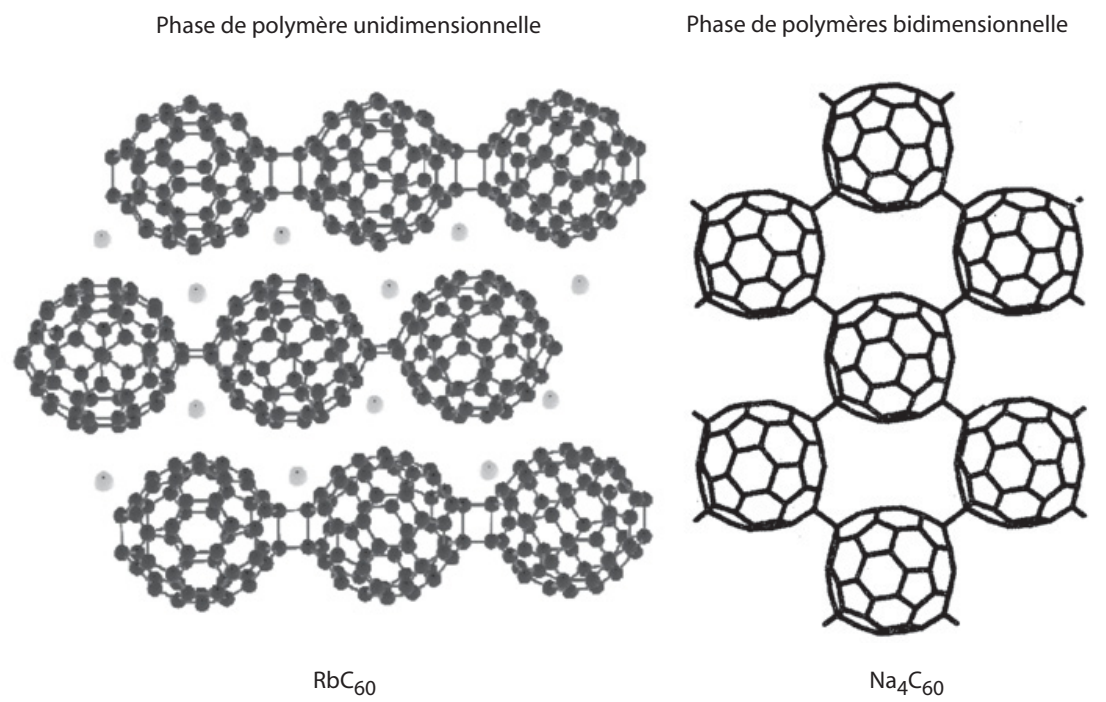

Figure 38. Représentation schématique de deux phases de polymère du $\mathrm{C}_{60}[32,33]$.

Dans la figure 39, nous montrons la densité d'états pour le $\mathrm{C}_{60}$ pur. On distingue les régions des modes externes et internes, séparés par un gap prononcé entre 6 et $30 \mathrm{meV}$. Les modes externes montrent une très faible dépendance vis à vis de la fréquence du vecteur d'onde $\vec{k}$. Néanmoins celleci n'est pas nulle comme le montrent les calculs. C'est exactement le comportement attendu pour un cristal moléculaire. En comparant la densité d'états du composé pur avec celle du composé $\mathrm{K}_{3} \mathrm{C}_{60}$ supraconducteur, on observe que les modes internes de haute fréquence réagissent fortement au transfert de charge. On peut conclure de cette observation que ces modes sont de bons candidats pour un couplage électron-phonon a priori très intense. Un tel couplage permettrait d'expliquer l'origine de la supraconductivité à température élevée dans ces systèmes.

Le gap est un signe direct de la séparation des échelles d'énergies qui détermine d'une part, la cohésion d'une cage isolée, et de l'autre la cohésion d'un ensemble de cages. Dans le cas des phases polymères du $\mathrm{C}_{60}$, la liaison inter-cages est covalente par nature ce qui influence les spectres essentiellement de deux façons : d'un coté, cette liaison induit une déformation des cages et provoque ainsi des changements subtils dans la fréquence des modes internes dont l'interprétation exige des calculs théoriques évolués; d'un autre coté, le système perd plus ou moins son caractère de cristal moléculaire, aboutissant à une variation de la densité spectrale dans la région du gap $(0-30 \mathrm{meV}) \mathrm{du}$ composé monomère (voir figure 40). L'analyse de ces modes de gap est quasiment directe tant qu'il n’y a pas de fort mélange entre les degrés de liberté internes et externes. Bien que les modes de gap correspondent à des excitations collectives, leurs fréquences sont déterminées principalement par l'environnement immédiat des molécules. Par conséquent, on peut obtenir relativement aisément des informations sur le type de liaison et la force des liaisons à partir des données spectroscopiques, même en présence de désordre à longue distance tels que des défauts d'empilement.

Naturellement, les calculs de dynamique qui donnent accès aux courbes de dispersion, facilitent l'interprétation et permettent d'aller encore plus loin dans l'analyse. Nous en montrons deux exemples pour les phases polymère et dimère $d u \mathrm{RbC}_{60}$ sur la figure 41 . Le spectre du dimère présente des pics bien nets autour de $10 \mathrm{meV}$ qui sont dûs aux modes internes des haltères de $\left(\mathrm{C}_{60}\right)_{2}$. Comme les haltères interagissent au moyen de faibles forces de Van der Waals, elles peuvent être considérées comme dynamiquement isolées les unes des autres en première approximation. Les modes de déformation des 

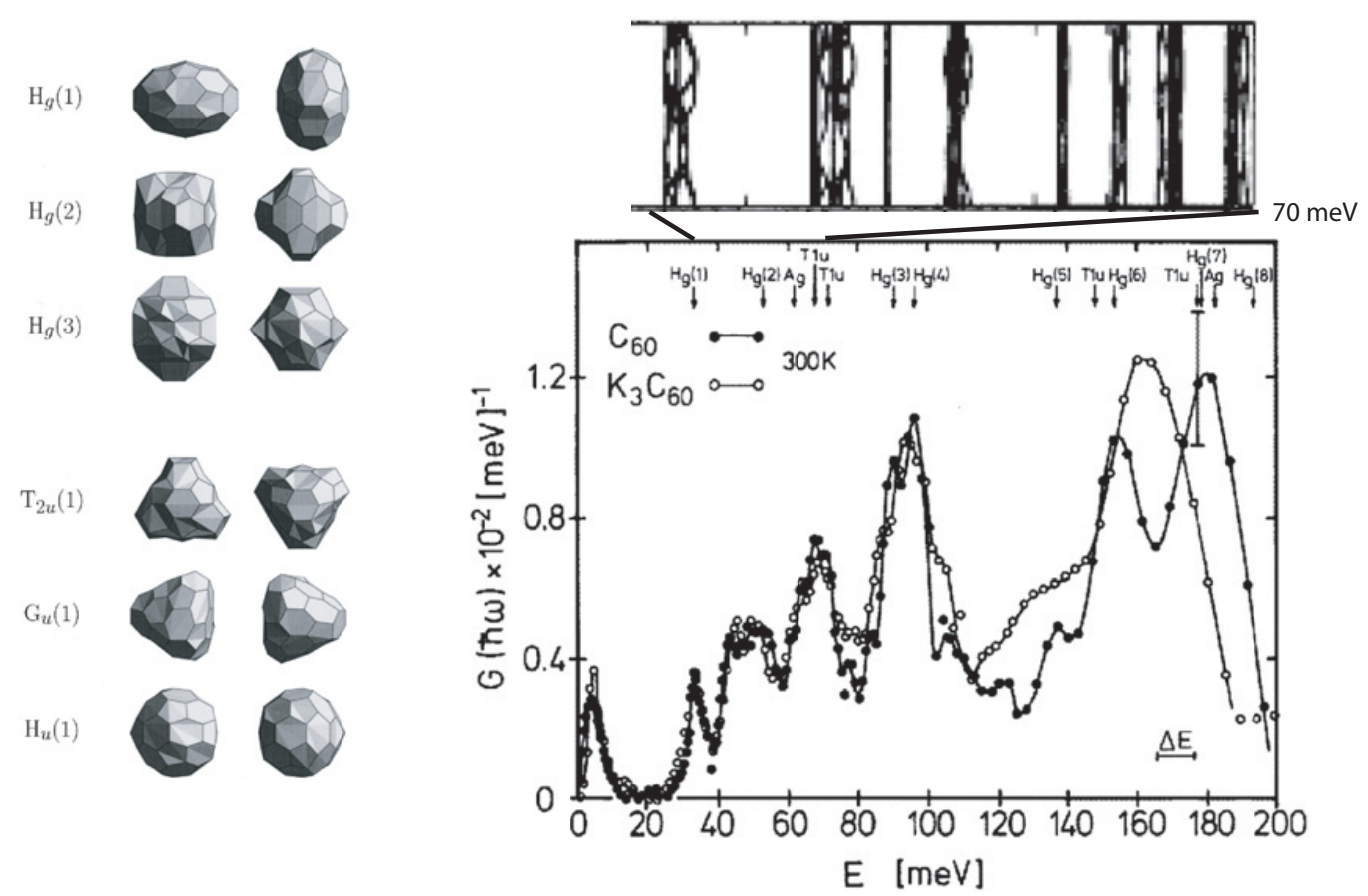

Figure 39. Spectre total du $\mathrm{C}_{60}$ et comparaison avec le supraconducteur $\mathrm{K}_{3} \mathrm{C}_{60}$. Les densités d'états ont été obtenues par diffusion des neutrons [31]. En tant que cristal moléculaire, le $\mathrm{C}_{60}$ possède 6 degrés de liberté externes (3 rotations plus 3 translations par molécule). Les phonons correspondants ont des fréquences de moins de $8 \mathrm{meV}$. Le reste des excitations correspond à des vibrations internes de la molécule. Elles occupent une fenêtre spectrale qui s'étend de 30 à $200 \mathrm{meV}$. Les flèches indiquent les modes de la molécule en centre de zone, déterminés par spectroscopie optique. Les symboles correspondent aux différentes représentations irréductibles des vibrations moléculaires. Les schémas à gauche montrent le type de déformation lié à ces représentations. Celles de type radial sollicitent relativement peu les liaisons entre carbones. Un exemple typique est le mode $\mathrm{H}_{g}(1)$, qui déforme le ballon en cigare. Les modes de type tangentiel, par contre, mènent à une forte déformation des pentagones et des hexagones. On les trouve par conséquent à haute fréquence. Modes externes et modes internes sont séparés d'un gap de $20 \mathrm{meV}$. Comme le démontrent les calculs, chaque branche de dispersion possède, en dépit de cette séparation forte des échelles d'énergie, une certaine largeur en fréquence, due au couplage entre molécules voisines. Cette largeur de bande est néanmoins très faible par rapport aux fréquences elles-mêmes (voir discussion en section 3.3.3). Le spectre du composé dopé est nettement différent du spectre du $\mathrm{C}_{60}$ pur. Ceci indique que le transfert de 3 électrons altère considérablement la dynamique de la molécule. Nous avons donc affaire à un couplage électronphonon fort, en particulier en ce qui concerne les vibrations de très haute fréquence. On reviendra sur ce point en section 4.4 .

haltères ont alors des dispersions plates ${ }^{82}$ et apparaissent sous la forme de pics fins dans le spectre de la densité d'états. On attribue la bande de fréquence la plus élevée aux librations des cages autour d'axes perpendiculaires aux haltères. La bande de plus basse fréquence correspond au mode d'étirement, équivalent moléculaire de la vibration d'une molécule diatomique. La torsion de l'haltère autour de son axe principal met seulement en jeu des forces de rappel relativement faibles. Elle se trouve ainsi cachée dans la bande de basse fréquence des modes intermoléculaires. Le spectre acoustique est significativement plus mou que dans le polymère, comme on doit s'y attendre pour un système moléculaire d'haltères uniquement liées par des forces de Van der Waals. Nous rappelons que ce sont les liaisons les plus faibles dans une chaîne qui déterminent sa rigidité aux grandes longueurs d'onde.

82 On les appelle également “modes d'Einstein”. 

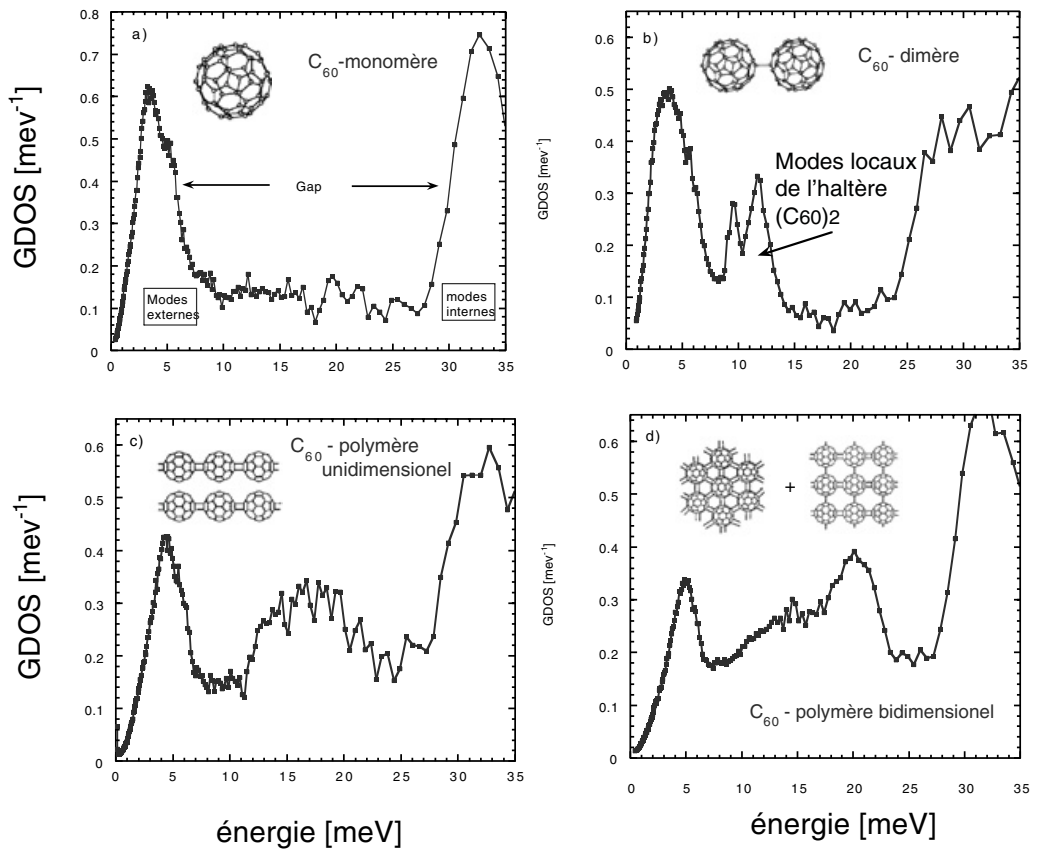

Figure 40. Densité d'état phononique dans la région des basses fréquences de diverses phases du $\mathrm{C}_{60}$. Les modes externes sont séparés des modes internes par un gap de $\approx 20 \mathrm{meV}$ dans la phase monomère. Dès que des liaisons se développent entre les molécules, le gap se remplit. Le poids spectral dans la région du gap augmente à fur et à mesure qu'on augmente la connectivité entre les molécules, et cela au détriment des modes externes.

Déjà qualitativement, le spectre de la phase polymère unidimensionnelle diffère de celui de la phase dimère. Par suite de la connectivité infinie du réseau suivant les directions de polymérisation, les branches de phonons externes montrent une dispersion, comme dans le cas du monomère, responsables de l'élargissement des bandes correspondantes dans le spectre de la densité d'états. Il est évident que le réseau à connexions tri-dimensionnelles du système polymère, à part des changements relativement faibles dans la matrice d'interaction, peut être considéré comme semblable au système monomère, c'està-dire que les spectres des modes externes sont reliés par une "mise à l'échelle" de l'axe des fréquences.

\section{AU-DELÀ DE L'APPROXIMATION HARMONIQUE}

L'approximation harmonique est un outil très performant pour étudier les excitations dans les solides. En général, l'hypothèse que les ions restent proches de leurs positions d'équilibre est valable dans la plupart des matériaux en dessous de leurs points de fusion. Des exceptions existent et nous comptons parmi elles les cristaux quantiques comme le $\mathrm{H}_{2}$ et $\mathrm{D}_{2}$ solide. En dépit de ses qualités indéniables, la description harmonique des solides souffre de son incapacité à expliquer des phenomènes assez courants comme l'expansion thermique des matériaux. Il est très instructif d'identifier la raison de cette défaillance du modèle.

\subsection{Expansion thermique et approximation quasi-harmonique}

Si le système étudié présente une expansion thermique, il faut appliquer une pression dépendante de la température pour maintenir le volume constant. Nous nous proposons de calculer cette pression à partir 

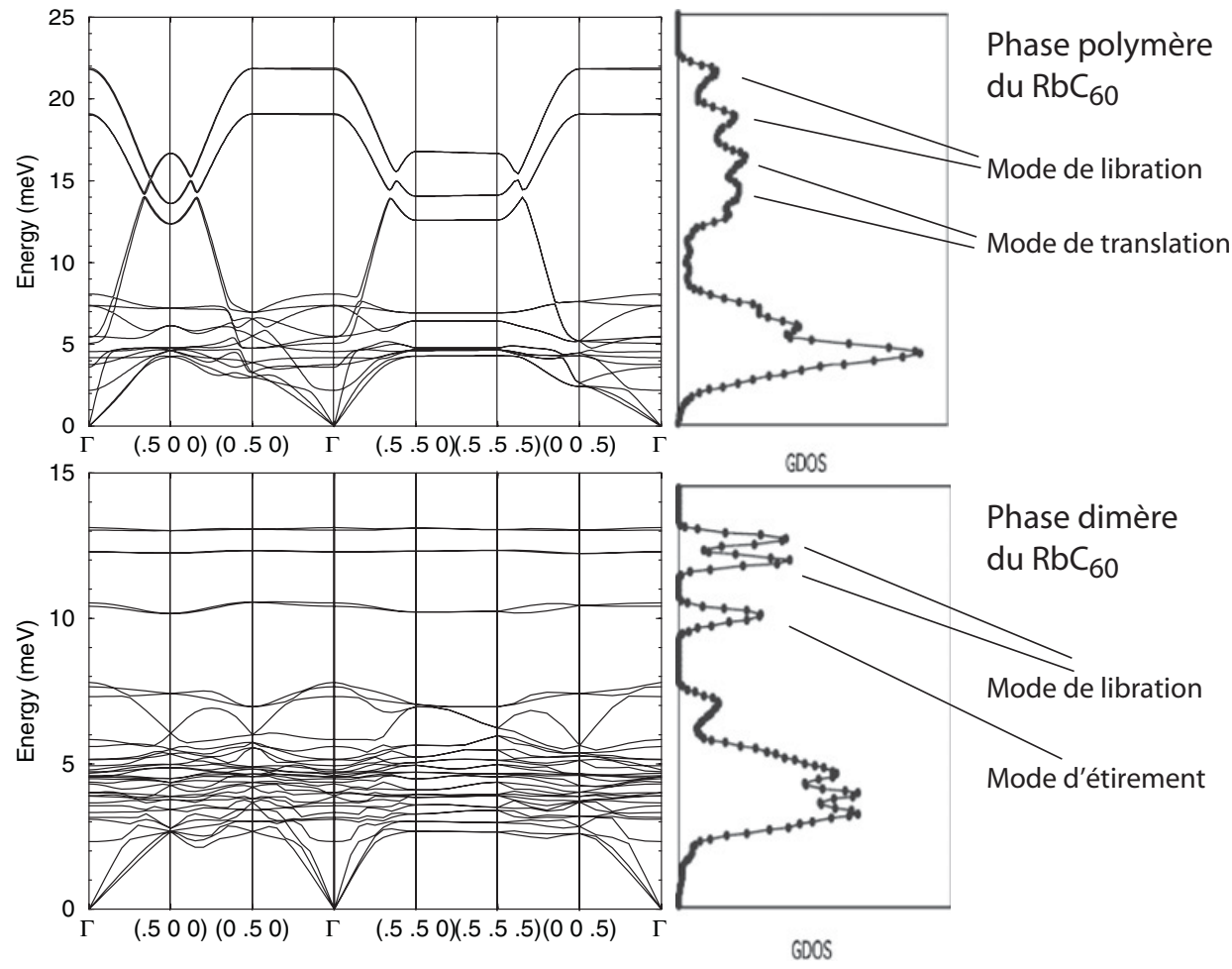

Figure 41. Courbes de dispersion et densités d'états $g(\omega)$ déduites pour la phase polymère et dimère du $\mathrm{RbC}_{60}$, respectivement [32]. Nous avons employé un modèle hybride combinant des champs de force et des interactions atome-atome dérivées d'un potentiel. Dans ces calculs, tous les atomes de carbone sont pris comme des unités dynamiques indépendantes. Les interactions $\mathrm{C}-\mathrm{C}$ dans les boules sont décrites par un champ de force. On introduit des potentiels atome-atome de Van der Waals pour les interactions $\mathrm{C}-\mathrm{A}(\mathrm{A}=\mathrm{Na}, \mathrm{K}, \mathrm{Rb}, \mathrm{Cs})$ ainsi que pour les liaisons entre deux atomes de carbone appartenant à deux molécules différentes. Les forces de rappel dans l'unité cyclo-butane $\mathrm{C}_{4}$, qui créent les liaisons chimiques entre boules dans la phase polymère, peuvent se déduire directement à partir d'interactions équivalentes sur la cage, confirmant le caractère fortement covalent de ces liaisons. Les forces de rappel angulaires pour les librations autour d'axes perpendiculaires à la direction de la chaîne sont très importantes, ce qui aboutit à des fréquences de libration au dessus de la bande d'étirement de la chaîne. D'un autre coté, les chaînes ne sont pas très rigides si on considère leur torsion. Concernant la relation de dispersion pour la phase dimère $\mathrm{RbC}_{60}$, on constate que les branches optiques sont plates ce qui entraîne des pics étroits dans la densité d'états. Les branches de plus haute fréquence correspondent aux librations autour d'axes perpendiculaires aux axes longs des haltères. On trouve les modes d'étirement des haltères dans la région des $10 \mathrm{meV}$. La modélisation de la dynamique des dimères est basée sur une liaison impliquant un contact entre deux atomes seulement. Une liaison type $\mathrm{C}_{4}$ comme dans le polymère exigerait des constantes de force trop basses d'un point de vue physique.

des énergies d'excitation du système. La relation thermodynamique

$$
p=-\left(\frac{\partial F}{\partial V}\right)_{T}
$$

relie la pression $p$ à l'énergie libre $F=U-T S$. L'énergie interne étant une fonction directe des énergies d'excitation, nous avons atteint notre but si nous parvenons à exprimer l'entropie en fonction de l'énergie interne. Ceci est possible en exploitant la relation

$$
C_{V}=T\left(\frac{\partial S}{\partial T}\right)_{V}=\left(\frac{\partial U}{\partial T}\right)_{V} .
$$


Nous obtenons

$$
p=-\frac{\partial}{\partial V}\left(U-T \int_{0}^{T} \frac{d T^{\prime}}{T^{\prime}} \frac{\partial}{\partial T^{\prime}} U\left(T^{\prime}, V\right)\right) .
$$

Cette expression nous permet de calculer la pression si nous connaissons la façon dont l'énergie interne $U$ dépend de la température et du volume. Dans l'approximation harmonique, celle-ci est donnée par

$$
U=U_{\mathrm{eq}}+\frac{1}{2} \sum_{\vec{k}, j}^{B Z, 3 r} \hbar \omega_{j}(\vec{k})+\sum_{\vec{k}, j}^{B Z, 3 r} \frac{\hbar \omega_{j}(\vec{k})}{\exp \left(\beta \hbar \omega_{j}(\vec{k})\right)-1} .
$$

La pression se calcule par conséquent comme ${ }^{83}$

$$
p=-\frac{\partial}{\partial V}\left\{U_{\text {eq }}+\frac{1}{2} \sum_{\vec{k}, j}^{B Z, 3 r} \hbar \omega_{j}(\vec{k})\right\}-\hbar\left\{\sum_{\vec{k}, j}^{B Z, 3 r} \frac{1}{\exp \left(\beta \hbar \omega_{j}(\vec{k})\right)-1}\left(\frac{\partial}{\partial V} \omega_{j}(\vec{k})\right)\right\} .
$$

Le premier terme dans cette équation est indépendant de la température. Il survit également à $T=0$ et ne nous intéresse pas dans le contexte de l'expansion thermique. Le second terme est une somme sur les dérivées des fréquences des modes par rapport au volume. Chaque contribution est ponderée par le facteur d'occupation. Plus la fréquence d'un mode change avec le volume plus celui-ci contribue à la pression.

Dans l'approximation harmonique, les fréquences ne dépendent pas du volume. Ceci peut paraitre contre-intuitif. Il se comprend néanmoins facilement par analogie avec le système mécanique. Les forces de rappel sont les mêmes, que les ressorts soient précontraints ou étirés, ou qu'ils soient en équilibre. Cette assertion est valable pourvu que la loi de Hooke reste applicable, c'est-à-dire pourvu qu'on se trouve dans le régime élastique des ressorts.

Le fait que la pression nécessaire pour maintenir le système à volume constant ne change pas avec la température implique selon

$$
\left(\frac{\partial V}{\partial T}\right)_{p}=-\left(\frac{\partial p}{\partial T}\right)_{V}\left(\frac{\partial p}{\partial V}\right)_{T}^{-1}
$$

qu'un système harmonique ne possède pas d'expansion thermique. Nous aurions pu obtenir ce résultat plus facilement en observant que l'occupation des phonons ne change pas la positions d'équilibre des ions. Un potentiel harmonique est totalement symétrique par rapport au rapprochement ou à l'éloignement de deux particules. Les forces de rappel sont identiques dans les deux cas. Les ions ne sont par conséquent pas incités à changer leurs distances (voir figure 42). La procédure choisie a l'avantage de pouvoir être généralisée aux systèmes anharmoniques.

Nous savons par expérience que les matériaux se dilatent. Le fait que cette tendance puisse être inversée, donnant des matériaux qui se contractent en augmentant la température, est moins connu. Nous devons en conclure que les énergies des excitations ne sont pas insensibles au volume. Dans le fond, ce constat ne nous étonne pas. Deux ions qu'on rapproche par la force, se repousseront de plus en plus fortement. Ils se comporteront comme des ressorts en fin de course. Les fréquences des oscillations augmenteront. On observera l'effet contraire pour des ions qu'on sépare. Pour un système couplé, la situation est plus complexe et rien n'exclut qu'il se contracte en le comprimant. C'est en phénomène bien connu dans la glace.

\footnotetext{
83 Ce résultat ne dépend pas de la symétrie de translation. Dans le cas des amorphes, la somme sur les vecteurs d'onde $\vec{k}$ est à remplacer par une seule somme sur les modes qui court de $j=1$ à $j=3 N$.
} 


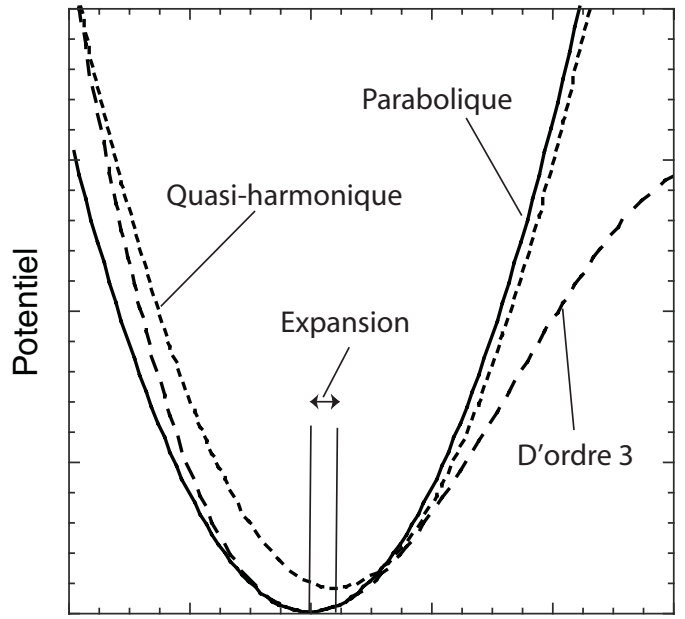

Position

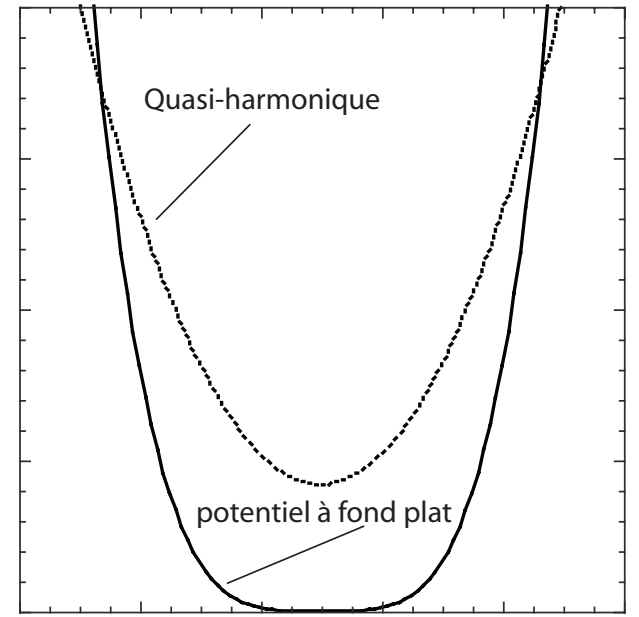

Position

Figure 42. Explication schématique de l'approximation quasi-harmonique. Image de gauche: à basse température, la parabole épouse le potentiel interionique dans son minimum. En augmentant la température, la distance entre particules change. La parabole est renormalisée pour décrire le mieux possible les forces de rappel autour de cette distance. La parabole devient plus large impliquant une baisse des fréquences. Image de droite : pour des particules en cage faiblement liées, le potentiel devient très plat à basse température. La fréquence baisse avec la température contrairement à la situation décrite dans l'image de gauche.

Du fait que la théorie harmonique est incapable de rendre compte de ces faits, nous avons besoin d'une extension. L'expansion thermique s'exprime par le coefficient d'expansion

$$
\alpha=\frac{1}{L}\left(\frac{\partial L}{\partial T}\right)_{p}=\frac{1}{3 V}\left(\frac{\partial V}{\partial T}\right)_{p}=\frac{1}{3 B}\left(\frac{\partial p}{\partial T}\right)_{V},
$$

où

$$
B=-V\left(\frac{\partial p}{\partial V}\right)_{T}
$$

est le module de compressibilité, et où nous avons supposé que le système est isotrope.

L'expansion thermique requiert, comme nous l'avons vu, de prendre en compte les termes anharmoniques dans l'expansion du potentiel ressenti par les ions. Elle est donc un très bon indicateur de l'harmonicité d'un matériau. Quand l'anharmonicité devient perceptible, l' Hamiltionien ne peut plus être diagonalisé formellement en introduisant les modes normaux. Le fait que les valeurs typiques des coefficients d'expansion $\alpha$ soient relativement petites dans les solides (de l'ordre de $10^{-4}$ à $10^{-6} \mathrm{~K}^{-1}$ ), nous montre comment sortir de l'impasse. L'expansion thermique peut être considerée comme une petite perturbation. L'approximation harmonique restera par conséquent valable mais il faudra renormaliser les valeurs propres. Les constantes de forces devront donc être réévaluées pour chaque jeux de positions d'équilibre, par exemple en fonction de la température. On parlera dans ce cas d'approximation quasiharmonique. C'est un peu comme travailler avec une chaîne linéaire à ressort progressifs et de prendre en compte l'évolution de la rigidité des ressorts en fonction de la précontrainte. Dans ce cas, l'Hamiltonien reste identique à l'Hamiltonien harmonique

$$
\mathbf{H}=\sum_{\vec{k}} \sum_{j=1}^{3 r} \hbar \omega_{j}(\vec{k} \mid V)\left(\mathbf{a}_{j}^{+}(\vec{k}) \mathbf{a}_{j}(\vec{k})+\frac{1}{2}\right) .
$$


avec la seule différence que les fréquences dépendent maintenant explicitement du volume. Ce résultat nous permet de calculer la dérivée de la pression par rapport à la température à volume constant en utilisant l'expression 4.5

$$
\left(\frac{\partial p}{\partial T}\right)_{V}=-\hbar \sum_{\vec{k}, j}^{B z, 3 r}\left(\frac{\partial}{\partial T} n_{j}(\vec{k})\right)\left(\frac{\partial}{\partial V} \omega_{j}(\vec{k})\right) .
$$

Nous rappelant que la contribution d'un mode harmonique à la chaleur spécifique est donnée par l'expression ${ }^{84}$

$$
c_{v}(j, \vec{k})=\frac{\hbar \omega_{j}(\vec{k})}{V}\left(\frac{\partial}{\partial T} n_{j}(\vec{k})\right)
$$

et en introduisant pour chaque mode les paramètres

$$
\gamma_{j}(\vec{k})=-\frac{V}{\omega_{j}(\vec{k})} \frac{\partial \omega_{j}(\vec{k})}{\partial V}=-\frac{\partial\left(\ln \omega_{j}(\vec{k})\right)}{\partial(\ln V)}
$$

nous obtenons pour les coefficients d'expansion thermique

$$
\alpha=\frac{1}{3 B} \sum_{\vec{k}, j}^{B z, 3 r} \gamma_{j}(\vec{k}) c_{v}(j \mid \vec{k})
$$

Les paramètres de Grüneisen $\gamma_{j}(\vec{k})$ représentent le changement relatif de la fréquence des oscillateurs par rapport au volume du système, c'est-à-dire le changement en pourcentage de la fréquence par changement en pourcentage du volume. Ces paramètres sont en général de l'ordre de l'unité. Un paramètre de Grüneisen de un, signifie que les fréquences sont reduites de moitié quand le volume double. Des paramètres négatifs s'obtiennent quand les fréquences augmentent avec le volume. Des modes de ce type peuvent, si leur influence n'est pas compensée par d'autres modes à comportement normal, produire une contraction du système avec la température. Les matériaux à expansion thermique négative ont reçu beaucoup d'attention ces dernières années à cause de leurs applications technologiques potentielles (voir figure 43 pour un exemple). Pour l'ingénieur, le matériau idéal est un matériau possédant une expansion thermique nulle sur une large gamme de température. Ce but pourrait être atteint en créant des matrices composées de matériaux avec des coefficients d'expansion négatifs et positifs.

Les paramètres de Grüneisen peuvent être calculés sans avoir recours à un traitement de perturbation. Deux approches sont possibles. Soit on détermine la dépendence des phonons par rapport au volume grâce à des calculs $a b$ initio [35]; soit à partir de champs de forces dans le cadre des modèles de dynamique de réseau [34]. Dans les deux cas la procédure consiste à faire d'abord varier le volume en changeant les constantes de maille. On équilibre ensuite la structure pour déterminer les positions des atomes en fonction du volume. Connaissant les positions d'équilibre, on peut déterminer les fréquences des phonons exactement comme on le ferait pour l'état fondamental à $V=V_{0}$.

\subsection{Interaction entre phonons}

Afin d'exprimer l'Hamiltonien dans l'approximation harmonique, nous avons développé le potentiel $U\left(\vec{R}_{1}, \ldots \vec{R}_{N}\right)$ en fonction des déplacements ioniques $\vec{u}(n \mid t)$ jusqu'à l'ordre deux. L'Hamiltonien harmonique obtenu sera noté $H^{(0)}$ dans la suite de ce paragraphe, afin d'insister sur le traitement perturbatif des termes d'ordre supérieur à 2 dits “anharmoniques”. Dans sa forme globale, l'Hamiltonien

\footnotetext{
84 C'est l'énergie du mode multipliée par son changement d'occupation par unité de volume. Cela correspond au changement d'énergie interne par intervalle de température.
} 


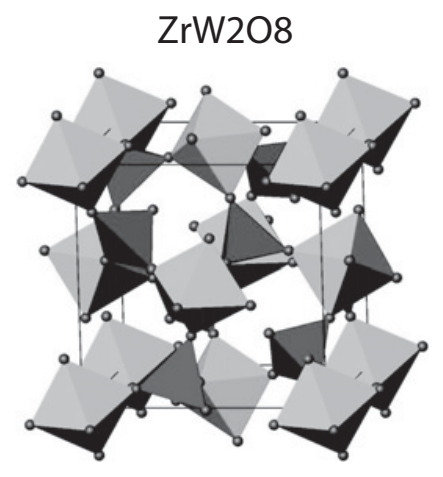

Expansion thermique

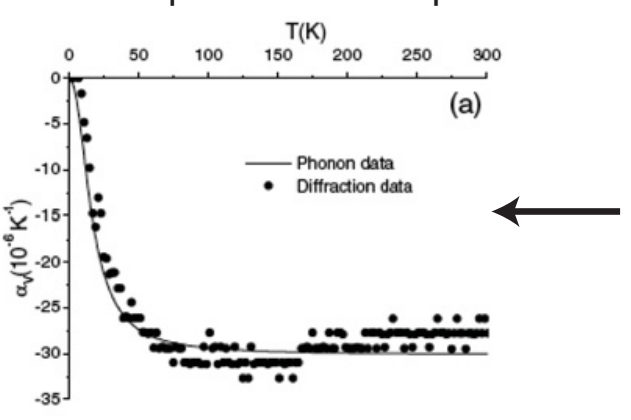

Evolution des spectres avec la pression
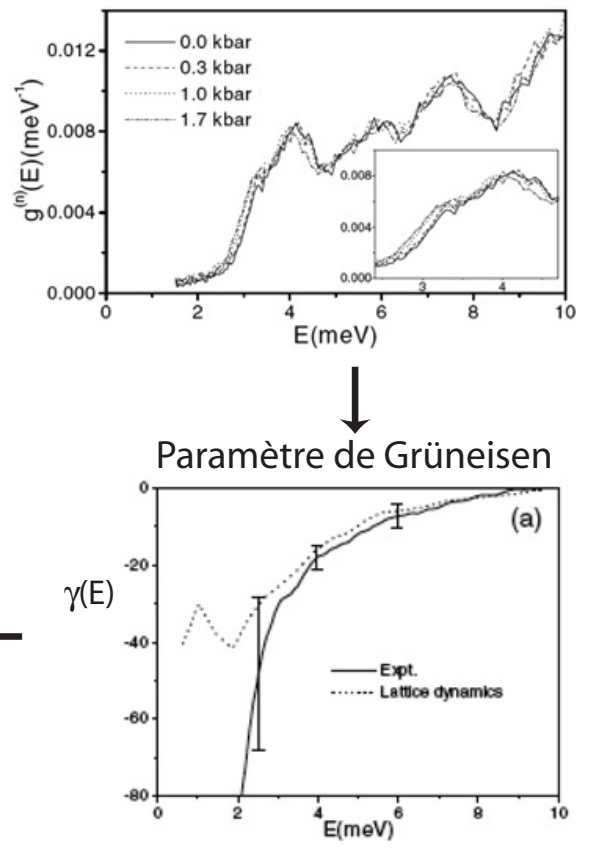

Figure 43. Exemple illustrant le lien entre phonons et expansion thermique. $\mathrm{ZrW}_{2} \mathrm{O}_{8}$ est un matériau céramique qui présente une expansion thermique négative sur une large gamme de températures. Comme le quartz, $\mathrm{ZrW}_{2} \mathrm{O}_{8}$ possède une charpente formée par des tétraèdres et des octaèdres liés par leurs sommets. Ce type de connectivité laisse beaucoup de flexibilité. Les modes de basse fréquence correspondent à des rotations d'unités rigides. Il est possible de déterminer la variation de la densité d'état des phonons en fonction de la pression en faisant appel à la diffusion des neutrons [34]. Connaissant la compressibilité du materiau, ces informations nous permettent de déduire la variation de $g(\omega)$ avec le volume. Cette variation s'exprime dans le paramètre de Grüneisen $\gamma(E)$, qui est intégré ici sur tous les modes qui partagent la même énergie. L'expansion thermique peut alors se calculer à l'aide de l'expression 4.13. Le résultat est en bonne adéquation avec les mesures de diffraction. La contraction thermique peut donc se comprendre à partir des phonons. En particulier, il est possible de déterminer les modes qui contribuent principalement à la contraction thermique. Leurs vecteurs propres nous renseignent sur les motifs de vibrations qui sont à l'origine de la contraction thermique.

peut ainsi s'écrire :

$$
H=H^{(0)}+U_{a n h}=H^{(0)}+U_{3}+U_{4}+\ldots
$$

Le premier terme anharmonique s'écrit comme

$$
U_{3}\left(\vec{R}_{1}, \ldots \vec{R}_{N}\right)=\left.\frac{1}{6} \sum_{\alpha, n=1}^{3, N} \sum_{\beta, n^{\prime}=1}^{3, N} \sum_{\gamma, n^{\prime \prime}=1}^{3, N} \frac{\partial^{3} U}{\partial u_{\alpha}(n) \partial u_{\beta}\left(n^{\prime}\right) \partial u_{\gamma}\left(n^{\prime \prime}\right)}\right|_{0} u_{\alpha}(n) u_{\beta}\left(n^{\prime}\right) u_{\gamma}\left(n^{\prime \prime}\right)
$$

ou dans la notation d'un système cristalin

$$
U_{3}\left(\vec{R}_{1}, \ldots \vec{R}_{N}\right)=\frac{1}{6} \sum_{\alpha, \kappa, \vec{l}} \sum_{\beta, \kappa^{\prime}, \vec{l}^{\prime}} \sum_{\gamma, \kappa^{\prime \prime}, \vec{l}^{\prime \prime}} \Phi_{\alpha \beta \gamma}\left(\begin{array}{ccc}
\vec{l} & \vec{l}^{\prime} & \vec{l}^{\prime \prime} \\
\kappa & \kappa^{\prime} & \kappa^{\prime \prime}
\end{array}\right) u_{\alpha}(\kappa, \vec{l}) u_{\beta}\left(\kappa^{\prime}, \vec{l}^{\prime}\right) u_{\gamma}\left(\kappa^{\prime \prime}, \vec{l}^{\prime \prime}\right)
$$



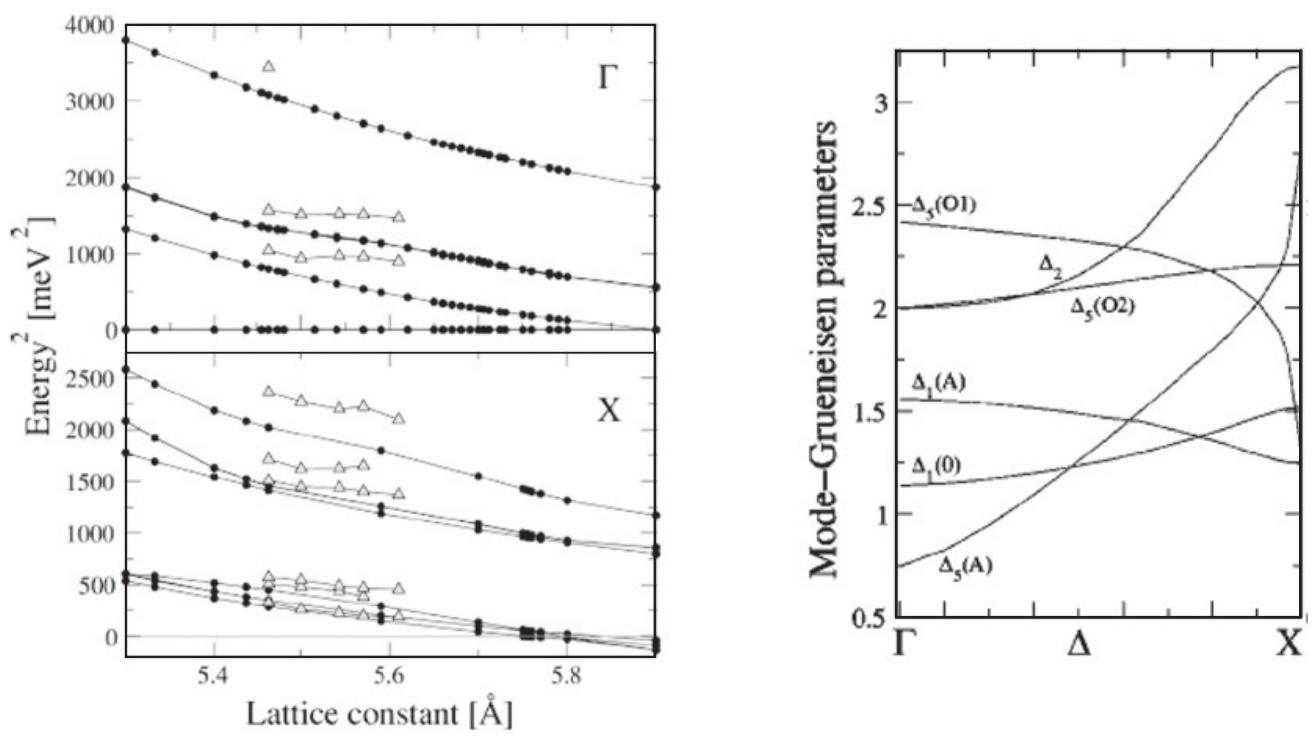

Figure 44. Evolution des fréquences avec le volume pour le $\mathrm{CaF}_{2}$ (voir figure 31) pour une sélection de modes. Les résultats sont obtenus avec des calculs ab initio [35]. Les triangles dans la figure de gauche indiquent les valeurs expérimentales. Les paramètres de Grüneisen dépendent du vecteur d'onde comme on peut le voir sur la figure de droite.

avec

$$
\Phi_{\alpha \beta \gamma}\left(\begin{array}{lll}
\vec{l} & \overrightarrow{l^{\prime}} & \overrightarrow{l^{\prime \prime}} \\
\kappa & \kappa^{\prime} & \kappa^{\prime \prime}
\end{array}\right)=\left.\frac{\partial^{3} U}{\partial u_{\alpha}(\kappa, \vec{l}) \partial u_{\beta}\left(\kappa^{\prime}, \overrightarrow{l^{\prime}}\right) \partial u_{\gamma}\left(\kappa^{\prime \prime}, \vec{l}^{\prime \prime}\right)}\right|_{0}
$$

Comme pour les constantes de forces d'ordre 2 la symétrie de translation impose une règle de somme à ces constantes d'ordre 3

$$
\sum_{\kappa, \vec{l}} \Phi_{\alpha \beta \gamma}\left(\begin{array}{lll}
\vec{l} & \vec{l} & \vec{l}^{\prime \prime} \\
\kappa & \kappa^{\prime} & \kappa^{\prime \prime}
\end{array}\right)=0
$$

En principe, il devrait être suffisant de ne considérer que l'ordre prépondérant dans le développement du potentiel. Il y a deux raisons pour lesquelles les termes d'ordre quatre peuvent devenir importants. En n'incluant que les termes d'ordre trois le problème devient mathématiquement mal défini. Nous ne pouvons pas aller dans les détails. Nous constatons simplement que l'énergie potentielle peut être rendue arbitrairement négative pour un potentiel de ce type. En dehors de cette complication mathématique, qui dans la pratique peut être contournée, les termes d'ordre trois sont souvent limités par des lois de conservation et quelquefois strictement nuls à cause de la symétrie des sites. On reviendra sur ce point plus loin. Par conséquent, il est important d'inclure les termes d'ordre quatre.

Etant donné que nous devons considérer les termes anharmoniques comme des petites perturbations du système harmonique, nous devons reformuler le problème dans le langage des modes normaux. Cette transition peut être effectuée en utilisant l'expression 3.223

$$
\mathbf{u}_{\alpha}(\kappa, \vec{l})=\sqrt{\frac{r}{N}} \sum_{j, \vec{k}} \sqrt{\frac{\hbar}{2 m_{k} \omega_{j}(\vec{k})}} e_{j}(\alpha, \kappa \mid \vec{k})\left(\mathbf{a}_{j}(\vec{k})+\mathbf{a}_{j}^{+}(-\vec{k})\right) e^{i \vec{k} \cdot \vec{l}}
$$


qui relie les opérateurs de déplacements des ions aux opérateurs de création et d'annihilation de phonons. Remplaçant les $u_{\alpha}(\kappa, \vec{l})$ dans $U_{3}\left(\vec{R}_{1}, \ldots \vec{R}_{N}\right)$, nous obtenons

$$
\begin{aligned}
U_{3}= & \frac{1}{6} \sum_{j_{1}, \vec{k}_{1}} \sum_{j_{2}, \vec{k}_{2}} \sum_{j_{3}, \vec{k}_{3}} V_{3}\left(j_{1}, j_{2}, j_{3} \mid \vec{k}_{1}, \vec{k}_{2}, \vec{k}_{3}\right) \\
& \times\left(\mathbf{a}_{j_{1}}\left(\vec{k}_{1}\right)+\mathbf{a}_{j_{1}}^{+}\left(-\vec{k}_{1}\right)\right)\left(\mathbf{a}_{j_{2}}\left(\vec{k}_{2}\right)+\mathbf{a}_{j_{2}}^{+}\left(-\vec{k}_{2}\right)\right)\left(\mathbf{a}_{j_{3}}\left(\vec{k}_{3}\right)+\mathbf{a}_{j_{3}}^{+}\left(-\vec{k}_{3}\right)\right)
\end{aligned}
$$

Les coefficients $V_{3}\left(j_{1}, j_{2}, j_{3} \mid \vec{k}_{1}, \vec{k}_{2}, \vec{k}_{3}\right)$ sont donnés par l'expression

$$
\begin{aligned}
& V_{3}\left(j_{1}, j_{2}, j_{3} \mid \vec{k}_{1}, \vec{k}_{2}, \vec{k}_{3}\right) \\
= & \left(\hbar \frac{r}{N}\right)^{\frac{3}{2}} \sum_{\alpha, \kappa} \sum_{\alpha, \kappa^{\prime}} \sum_{\alpha, \kappa^{\prime \prime}} \sum_{\vec{l}, \vec{l}^{\prime}, \vec{l}^{\prime \prime}} \frac{1}{\sqrt{m_{\kappa} m_{\kappa^{\prime}} m_{\kappa^{\prime \prime}}}} \frac{1}{\sqrt{8 \omega_{j_{1}}\left(\vec{k}_{1}\right) \omega_{j_{2}}\left(\vec{k}_{2}\right) \omega_{j_{3}}\left(\vec{k}_{3}\right)}} \\
& \times \Phi_{\alpha \beta \gamma}\left(\begin{array}{ccc}
\vec{l} & \vec{l}^{\prime} & \vec{l}^{\prime \prime} \\
\kappa & \kappa^{\prime} & \kappa^{\prime \prime}
\end{array}\right) \\
& \times e_{j_{1}}\left(\alpha, \kappa \mid \vec{k}_{1}\right) e_{j_{2}}\left(\alpha^{\prime}, \kappa^{\prime} \mid \vec{k}_{2}\right) e_{j_{3}}\left(\alpha^{\prime \prime}, \kappa^{\prime \prime} \mid \vec{k}_{3}\right) \times e^{i \vec{k}_{1} \cdot \vec{l}} e^{i \vec{k}_{2} \cdot \vec{l}^{\prime}} e^{i \vec{k}_{3} \cdot \vec{l}^{\prime \prime}} .
\end{aligned}
$$

Ils peuvent être considérés comme les transformées de Fourier des constantes de force d'ordre 3. Part la propriété

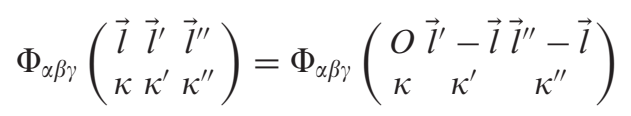

ces coefficients s'expriment également comme

$$
\begin{aligned}
& V_{3}\left(j_{1}, j_{2}, j_{3} \mid \vec{k}_{1}, \vec{k}_{2}, \vec{k}_{3}\right) \\
= & \left(\hbar^{3} \frac{r}{N}\right)^{\frac{1}{2}} \sum_{\alpha, \kappa} \sum_{\alpha^{\prime}, \kappa^{\prime}} \sum_{\alpha^{\prime \prime}, \kappa^{\prime \prime}} \sum_{\vec{l}^{\prime}, \vec{l}^{\prime \prime}} \frac{1}{\sqrt{m_{\kappa} m_{\kappa^{\prime}} m_{\kappa^{\prime \prime}}}} \frac{1}{\sqrt{8 \omega_{j_{1}}\left(\vec{k}_{1}\right) \omega_{j_{2}}\left(\vec{k}_{2}\right) \omega_{j_{3}}\left(\vec{k}_{3}\right)}} \\
& \times \Phi_{\alpha \beta \gamma}\left(\begin{array}{ccc}
O & \vec{l}^{\prime} & \vec{l}^{\prime \prime} \\
\kappa & \kappa^{\prime} & \kappa^{\prime \prime}
\end{array}\right) \\
& \times e_{j_{1}}\left(\alpha, \kappa \mid \vec{k}_{1}\right) e_{j_{2}}\left(\alpha^{\prime}, \kappa^{\prime} \mid \vec{k}_{2}\right) e_{j_{3}}\left(\alpha^{\prime \prime}, \kappa^{\prime \prime} \mid \vec{k}_{3}\right) \times e^{-i \vec{k}_{2} \cdot \vec{l}^{\prime}} e^{-i \vec{k}_{3} \cdot \vec{l}^{\prime \prime}} \\
& \times \Delta\left(\vec{k}_{1}+\vec{k}_{2}+\vec{k}_{3}\right) .
\end{aligned}
$$

Cette manière d'exprimer les coefficients est élégante car elle souligne la conservation des vecteurs $\vec{k}$ lors de processus anharmoniques.

En parfaite analogie, nous obtenons pour les termes d'ordre quatre

$$
\begin{aligned}
U_{4}= & \frac{1}{24} \sum_{j_{1}, \vec{k}_{1}} \sum_{j_{2}, \vec{k}_{2}} \sum_{j_{3}, \vec{k}_{3}} \sum_{j_{3}, \vec{k}_{3}} V_{4}\left(j_{1}, j_{2}, j_{3}, j_{4} \mid \vec{k}_{1}, \vec{k}_{2}, \vec{k}_{3}, \vec{k}_{4}\right) \\
& \times\left(\mathbf{a}_{j_{1}}\left(\vec{k}_{1}\right)+\mathbf{a}_{j_{1}}^{+}\left(-\vec{k}_{1}\right)\right)\left(\mathbf{a}_{j_{2}}\left(\vec{k}_{2}\right)+\mathbf{a}_{j_{2}}^{+}\left(-\vec{k}_{2}\right)\right)\left(\mathbf{a}_{j_{3}}\left(\vec{k}_{3}\right)+\mathbf{a}_{j_{3}}^{+}\left(-\vec{k}_{3}\right)\right)\left(\mathbf{a}_{j_{4}}\left(\vec{k}_{4}\right)+\mathbf{a}_{j_{4}}^{+}\left(-\vec{k}_{4}\right)\right) .
\end{aligned}
$$

Nous rappelant les difficultés qu'on rencontre quand on veut calculer les constantes de force harmoniques, il devient évident que les constantes de force d'ordre supérieur sont hors de notre portée, sauf pour des systèmes extrêmement simples. On peut néanmoins tirer beaucoup de renseignements 
sur les effets anharmoniques en étudiant la forme générale des processus anharmoniques à partir des expressions 4.20 et 4.24 .

Dans une théorie de perturbation, l'effet du potentiel anharmonique est de modifier les fonctions d'onde ainsi que les énergies propres. Nous ne considérons pour l'instant pas ces complications qui apparaissent quand les fonctions d'ondes sont dégénérées. Nous serons néanmoins obligés de revenir sur ce point plus tard. Soit $\left|n^{(0)}\right\rangle$ une fonction propre de l'Hamiltonien harmonique - c'est à dire l'Hamiltonien non perturbé - de la forme 3.240

$$
\left|n^{(0)}\right\rangle \equiv\left|n_{1}(\vec{k}), \ldots, n_{j}(\vec{k})\right\rangle=\prod_{j=1}^{3 r} \frac{\left(\hat{a}_{j}^{+}\right)^{n_{j}(\vec{k})}}{\sqrt{n_{j}(\vec{k}) !}}|0, \ldots, 0\rangle,
$$

avec l'énergie $E_{n}^{(0)}$. En utilisant le formalisme de la théorie des perturbations pour des systèmes dont les niveaux d'énergie sont non-dégénérés, la correction des valeurs propres au premier ordre sera

$$
E_{n}^{(1)}=\left\langle n^{(0)}\left|U_{\text {anh }}\right| n^{(0)}\right\rangle
$$

de sorte que les niveaux d'énergie s'expriment par $E_{n}=E_{n}^{(0)}+E_{n}^{(1)}$. Pour la fonction d'onde nous obtenons la correction

$$
\left|n^{(1)}\right\rangle=\sum_{m \neq n} \frac{\left\langle m^{(0)}\left|U_{\mathrm{anh}}\right| n^{(0)}\right\rangle}{E_{n}^{(0)}-E_{m}^{(0)}}\left|m^{(0)}\right\rangle .
$$

Dans cette expression la somme court sur tous les états harmoniques $\left|m^{(0)}\right\rangle$ différents de $\left|n^{(0)}\right\rangle$. Une fonction propre harmonique acquiert donc des contributions de toutes les autres fonctions d'onde harmoniques pour lesquelles l'élément de matrice $\left\langle m^{(0)}\left|U_{\text {anh }}\right| n^{(0)}\right\rangle$ est non-nul.

Le système anharmonique le plus simple est un oscillateur isolé dans un potentiel qui comporte en plus du terme harmonique en $x^{2}$ une correction en $x^{3}$. Il nous sert d'illustration du calcul de perturbation. L'Hamiltonien de cette oscillateur anharmonique s'écrit comme

$$
\mathbf{H}=\frac{\hbar \omega}{2}\left(\hat{\xi}^{2}+\hat{\pi}^{2}\right)+U_{\mathrm{anh}}\left(\hat{\xi}^{3}\right)=\hbar \omega\left(\frac{1}{2}+\hat{a}^{+} \hat{a}\right)+U_{\mathrm{anh}}\left(\hat{a}^{+}, \hat{a}\right)
$$

avec

$$
\begin{aligned}
U_{\mathrm{anh}}\left(\hat{a}^{+}, \hat{a}\right)=\epsilon(\hbar \omega) \xi^{3} & =\frac{\epsilon(\hbar \omega)}{(\sqrt{2})^{3}}\left(\mathbf{a}^{+^{3}}+\mathbf{a}^{3}+3\left(\mathbf{a}^{+} \mathbf{a}\right) \mathbf{a}^{+}+3\left(\mathbf{a}^{+} \mathbf{a}+1\right) \mathbf{a}\right) \\
& =\frac{\epsilon(\hbar \omega)}{(\sqrt{2})^{3}}\left(\mathbf{a}^{+3}+\mathbf{a}^{3}+3 \mathbf{N} \mathbf{a}^{+}+3(\mathbf{N}+1) \mathbf{a}\right),
\end{aligned}
$$

où nous avons utilisé les relations entre les opérateurs introduits en section 2.1.2. L'importance de la perturbation est régie par le paramètre $\epsilon$. Les éléments de matrice non-nuls se calculent comme

$$
\begin{aligned}
\left\langle n-1\left|U_{\text {anh }}\right| n\right\rangle & =3 \epsilon \sqrt{\left(\frac{n}{2}\right)^{3}} \hbar \omega, \\
\left\langle n+1\left|U_{\text {anh }}\right| n\right\rangle & =3 \epsilon \sqrt{\left(\frac{n+1}{2}\right)^{3}} \hbar \omega, \\
\left\langle n-3\left|U_{\text {anh }}\right| n\right\rangle & =\epsilon \sqrt{\frac{n(n-1)(n-2)}{8}} \hbar \omega, \\
\left\langle n+3\left|U_{\text {anh }}\right| n\right\rangle & =\epsilon \sqrt{\frac{(n+3)(n+2)(n+1)}{8}} \hbar \omega .
\end{aligned}
$$


Ceci nous permet de calculer les énergies corrigées ainsi que les fonctions d'onde corrigées. Etant donné que la perturbation, selon l'expression 4.29, soit augmente soit réduit le nombre d'occupation $n$, et que le produit scalaire entre deux états avec des $n$ différents est nul, il suit que

$$
\left\langle n\left|U_{\text {anh }}\right| n\right\rangle=0 .
$$

L'énergie ne change donc pas au premier ordre de perturbation. L'expression pour l'énergie au deuxième ordre de perturbation donne

$$
E_{n}=\hbar \omega\left\{\left(n+\frac{1}{2}\right)-\frac{15}{4} \epsilon^{2}\left(n+\frac{1}{2}\right)^{2}-\frac{7}{16} \epsilon^{2}\right\} .
$$

Tous les niveaux d'énergie sont donc réduits par rapport aux valeurs harmoniques. La renormalisation est plus forte pour les niveaux plus élevés. Même l'énergie fondamentale est concernée. On appelle ces corrections d'énergie "self-energy corrections". Les niveaux ne sont plus équidistants. Nous obtenons pour la différence entre niveaux adjacents

$$
E_{n}-E_{n-1}=\hbar \omega\left(1-\frac{15}{2} \epsilon^{2} n\right) .
$$

Remplaçant les éléments de matrice 4.30 dans l'expression 4.27, nous obtenons les fonctions d'onde corrigées

$$
\begin{aligned}
\left|n^{(1)}\right\rangle= & \left|n^{(0)}\right\rangle \\
& +3 \epsilon \sqrt{\left(\frac{n}{2}\right)^{3}} \mid(n-1)^{(0)\rangle} \\
& -3 \epsilon \sqrt{\left(\frac{n+1}{2}\right)^{3}} \mid(n+1)^{(0)\rangle} \\
& +\frac{\epsilon}{3} \sqrt{\frac{n(n-1)(n-2)}{8}}\left|(n-3)^{(0)}\right\rangle \\
& -\frac{\epsilon}{3} \sqrt{\frac{(n+3)(n+2)(n+1)}{8}}\left|(n+3)^{(0)}\right\rangle
\end{aligned}
$$

Chaque fonction d'onde corrigée contient donc en plus de la fonction harmonique correspondante $\left(\left|n^{(0)}\right\rangle\right.$ ), des contributions des fonctions d'ondes harmoniques des niveaux \pm 1 et \pm 3 . Pour démontrer les conséquences physiques de ces contributions, nous calculons la valeur moyenne de l'opérateur $\xi$ pour l'état fondamental

$$
\left\langle 0^{(1)}|\xi| 0^{(1)}\right\rangle=\frac{1}{\sqrt{2}}\left\langle 0^{(1)}\left|\left(a^{+}+a\right)\right| 0^{(1)}\right\rangle=-\frac{3}{4} \epsilon .
$$

Ceci correspond au déplacement de la position par rapport au minimum du potentiel. Dans l'exemple de la figure $42, \epsilon$ est négatif. La position d'équilibre de la fonction d'onde fondamentale se déplace donc vers des valeurs plus grandes de $x$. C'est bien compréhensible, étant donné que le potentiel est plus plat pour les grandes distances. Quand le nombre d'occupation monte avec la température, le décentrage s'accentue. L'expansion thermique du solide est donc un bon indicateur de l'anharmonicité.

Une autre conséquence de l'anharmonicité est la modification des règles de sélection pour l'interaction avec un champ électromagnétique. Dans l'approximation dipolaire, celles-ci sont régies 
a)

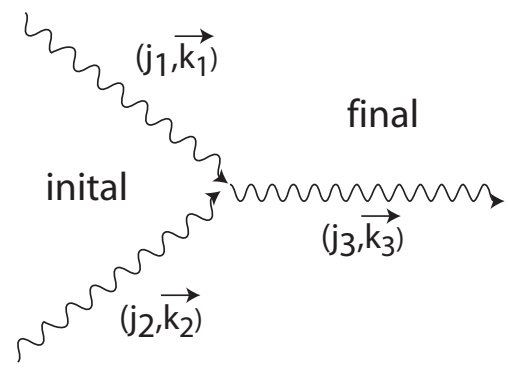

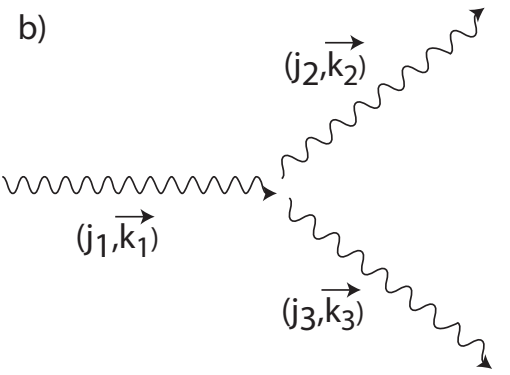

e) c)

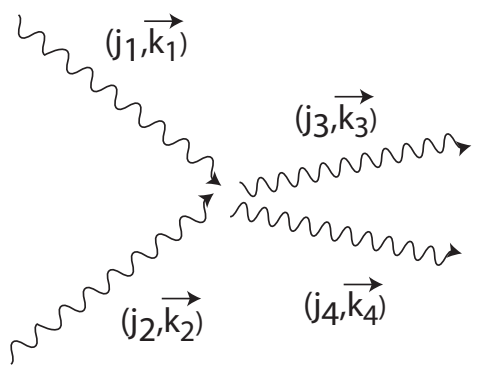

d)

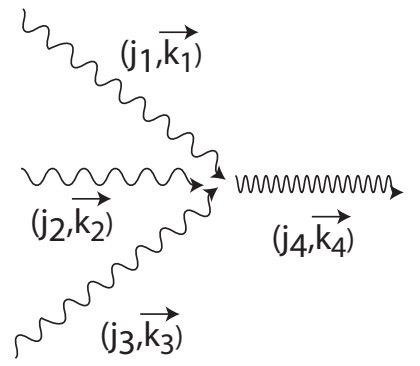

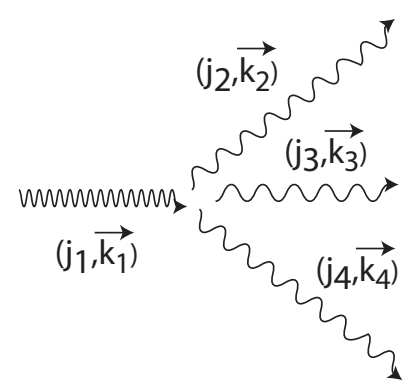

Figure 45. Représentation schématique des interactions entre phonons. Les termes anharmoniques d'ordre 3 contribuent aux processus présentés en haut de la figure. Ils correspondent à la fusion de deux phonons en un seul (a) ou à la désintégration d'un phonon en deux (b). Les processus d'ordre 4 sont au nombre de trois. Deux phonons se transforment en deux autres (c), trois phonons fusionnent en un seul (d) et un phonon se désintègre en trois. Tous ces processus doivent respecter la conservation de l'énergie et du moment cristallin (voir le texte).

par les éléments de matrice de l'opérateur $\xi$. Dans l'approximation harmonique, seuls les éléments

$$
\langle 1|\xi| 0\rangle=\frac{1}{\sqrt{2}}
$$

sont non nuls. En incluant les termes anharmoniques, on rajoute

$$
\left\langle 2^{(1)}|\xi| 0^{(1)}\right\rangle=\frac{1}{\sqrt{2}} \epsilon .
$$

On observera donc une ligne supplémentaire dans les spectres d'absorption (“overtone”) à une fréquence qui n'est que légèrement décalée par rapport au double de la ligne fondamentale.

Après cette petite excursion dans le monde de l'oscillateur anharmonique simple, revenons aux phonons et étudions la forme des éléments de matrice $\left\langle m^{(0)}\left|U_{\mathrm{anh}}\right| n^{(0)}\right\rangle$ qui gouvernent les corrections anharmoniques.

- Les termes anharmoniques d'ordre $q$ en $\xi$ sont des combinaisons linéaires de termes issus du produits de ${ }^{85} s \leq q$ opérateurs d'annihilation $\mathbf{a}_{j_{1}}\left(\vec{k}_{1}\right) \cdot \ldots \cdot \mathbf{a}_{j_{s}}\left(\vec{k}_{s}\right)$ par $q-s$ opérateurs de création $\mathbf{a}_{j_{s+1}}^{+}\left(\vec{k}_{s+1}\right) \cdot \ldots \cdot \mathbf{a}_{j_{q}}^{+}\left(\vec{k}_{q}\right)$. Les opérateurs d'annihilation réduiront de un les nombres d'occupation des phonons $\left(n_{j_{1}}\left(\vec{k}_{1}\right), \ldots, n_{j_{s}}\left(\vec{k}_{s}\right)\right)$ d'un état $\left|n^{(0)}\right\rangle$. Par conséquent ils donneront

\footnotetext{
85 Nous négligeons ici les termes où plus d'un opérateur concerne le même mode normal, c'est-à-dire tous les termes qui figurent dans l'expression 4.34 pour l'oscillateur isolé. Ce sont des cas rares pour un système macroscopique. Ils compliquent le formalisme parce que ces opérateurs ne commutent pas.
} 
un résultat nul si les phonons qui leur correspondent dans l'état $\left|n^{(0)}\right\rangle$ ne sont pas occupés. Par analogie, les $q-s$ opérateurs de création augmenteront l'occupation des phonons $\left(n_{j_{s+1}}\left(\vec{k}_{s+1}\right), \ldots, n_{j_{q}}\left(\vec{k}_{q}\right)\right)$ leur correspondant de un. Par conséquent, les éléments de matrice du terme d'anharmonicité d'ordre $q$ seront non nuls uniquement entre des états $\left|n^{(0)}\right\rangle$ et $\left|m^{(0)}\right\rangle$ dont $r$ nombres d'occupation changent ${ }^{86}$.

- Pour le terme d'ordre trois, cela signifie qu'une fonction d'onde harmonique se transforme suite à deux processus. Le premier implique la fusion de deux phonons en un seul et le deuxième la désintégration d'un phonon en deux. Ces processus sont présentés schématiquement sur la figure 45.

- Pour le terme anharmonique d'ordre quatre, on a en plus de la fusion de trois en un et de la désintégration de un en trois, la transformation de deux en deux (aussi appelée diffusion des phonons) à prendre en compte (voir figure 45).

L'expression 4.27 implique que les corrections dominantes proviennent des états avec une énergie proche de celle de l'état non-perturbé à cause du terme en

$$
\frac{1}{E_{n}^{(0)}-E_{m}^{(0)}} \text {. }
$$

Dans un cristal macroscopique, le niveau de dégénérescence pour une énergie donnée n'est pas négligeable. La théorie de perturbation pour états non-dégénerés n'est donc pas applicable, et surtout pas à $T=0 .{ }^{87}$ Une solution est proposée par la théorie quantique des champs [38]. Il est hors de question de discuter ce formalisme. Nous pouvons néanmoins faire les constats suivants.

1. Les processus contribuant aux corrections anharmoniques seront de la forme décrite dans la figure 45. On doit néanmoins rester prudent et ne pas prendre ces diagrammes comme de véritables événements de diffusion de particules (phonons) dans le temps. Ils doivent être évalués dans l'ensemble thermodynamique choisi. Les phonons sont un outil mathématique et leurs recombinaisons perpétuelles, une manière formelle de décrire un système qui n'est pas harmonique.

2. Sous l'influence du potentiel anharmonique, les dégénérescences des états harmoniques seront levées sauf celles qui sont imposées par la symétrie. Les niveaux pour un vecteur $\vec{k}$ donné se décomposeront et acquerront une largeur finie. La perturbation peut être considérée faible si la largeur de la bande des niveaux décomposés est faible par rapport à la séparation des surfaces de dispersion. Ceci implique qu'on n'aura plus affaire à un système de $3 r$ modes discrets par vecteur d'onde. Les largeurs des niveaux sont appelées largeurs intrinsèques ${ }^{88}$ des phonons.

3. Les processus de recombinaison seront ceux qui conservent l'énergie. En clair, un processus décrit par l'élément de matrice $\left\langle m^{(0)}\left|U_{\mathrm{anh}}\right| n^{(0)}\right\rangle$ ne contribue à la renormalisation de l'énergie ou à la décomposition du niveau, que si

$$
\sum_{j, \vec{k}} \hbar \omega_{j}(\vec{k}) n_{j}^{\text {init }}(\vec{k})=\sum_{j, \vec{k}} \hbar \omega_{j}(\vec{k}) n_{j}^{\text {final }}(\vec{k}),
$$

avec $n_{j}^{\text {init }}(\vec{k})$ et $n_{j}^{\text {init }}(\vec{k})$ représentent les populations des phonons dans les états $\left|n^{(0)}\right\rangle$ et $\left|m^{(0)}\right\rangle$ respectivement ${ }^{89}$.

\footnotetext{
${ }^{86}$ Ceci implique que la correction des valeurs des niveaux d'énergie $\left(E^{(1)}\right)$ est nulle au premier ordre pour des niveaux non dégénérés. Elle n'intervient alors qu'au deuxième ordre des perturbations.

87 Dans le sous-espace des états dégénérés, aucune perturbation n'est malheureusement faible. Nous devons donc diagonaliser le potentiel anharmonique dans ce sous-espace pour décrire l'effet anharmonique. Cette tâche est d'une complexité analytique insurmontable.

88 Ils sont à distinguer des largeurs de bande qu'on obtient quant on projette une surface de dispersion sur l'axe d'énergie.

${ }^{89}$ Nous parlons d'un système à l'équilibre thermodynamique, c'est à dire que son énergie moyenne est constante.
} 


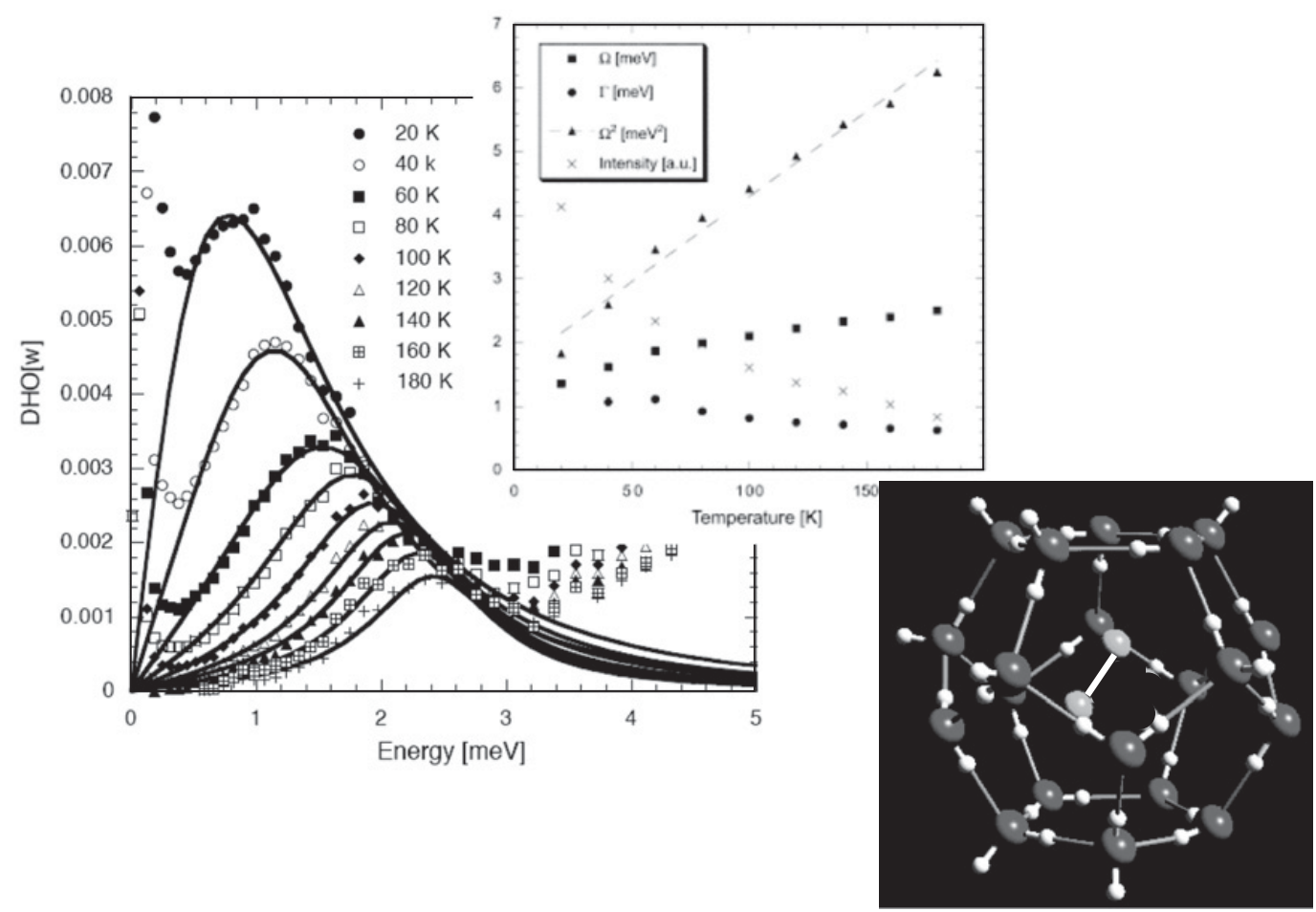

Figure 46. Les molécules de $\mathrm{N}_{2}$ remplissent les cages de clathrate en laissant de grandes parties vide. Le potentiel dans lequel elles se trouvent est donc très anharmonique. L'excitation ramollit fortement quand on baisse la température. Ce comportement anormal est contraire à celui de la plupart des phonons. La forme spectrale de l'excitation mesurée par diffusion inélastique des neutrons est bien décrite par un oscillateur amorti (se référer à 4.40). La fréquence au carré baisse linéairement avec la température tandis que la largeur augmente avec la température [37].

4. Les états propres de $U_{\text {anh }}$ peuvent toujours être étiquetés selon les représentations irréductibles du groupe des translations du cristal. Ceci implique la conservation du moment cristallin (se référer aussi au chapitre III). Par conséquent, un processus contribue uniquement si

$$
\sum_{j, \vec{k}} \vec{k} n_{j}^{\text {init }}(\vec{k})=\sum_{j, \vec{k}} \vec{k} n_{j}^{\text {final }}(\vec{k})+\vec{G}
$$

où $\vec{G}$ dénote un vecteur quelconque du réseau réciproque. Il est utile de rappeler ici que le moment cristallin n'a rien à voir avec l'impulsion.

Pour le spectroscopiste, on peut résumer la situation de la manière suivante : les processus anharmoniques ont principalement deux conséquences observables. Premièrement, les phonons pour un $\vec{k}$ donné acquièrent une largeur. Deuxièmement, leur fréquence centrale change avec la température. Les deux effets peuvent être décrits de façon satisfaisante en introduisant pour chaque mode un oscillateur amorti. La susceptibilité (ou plus précisément sa partie imaginaire) est donnée par l'expression [36]

$$
\chi_{(j, \vec{k})}^{\prime \prime}(\omega)=A \frac{4 \omega \Gamma(j, \vec{k}) \Omega(j, \vec{k})}{\left(\omega^{2}-\Omega(j, \vec{k})^{2}\right)^{2}-4 \omega^{2} \Gamma(j, \vec{k})^{2}} .
$$



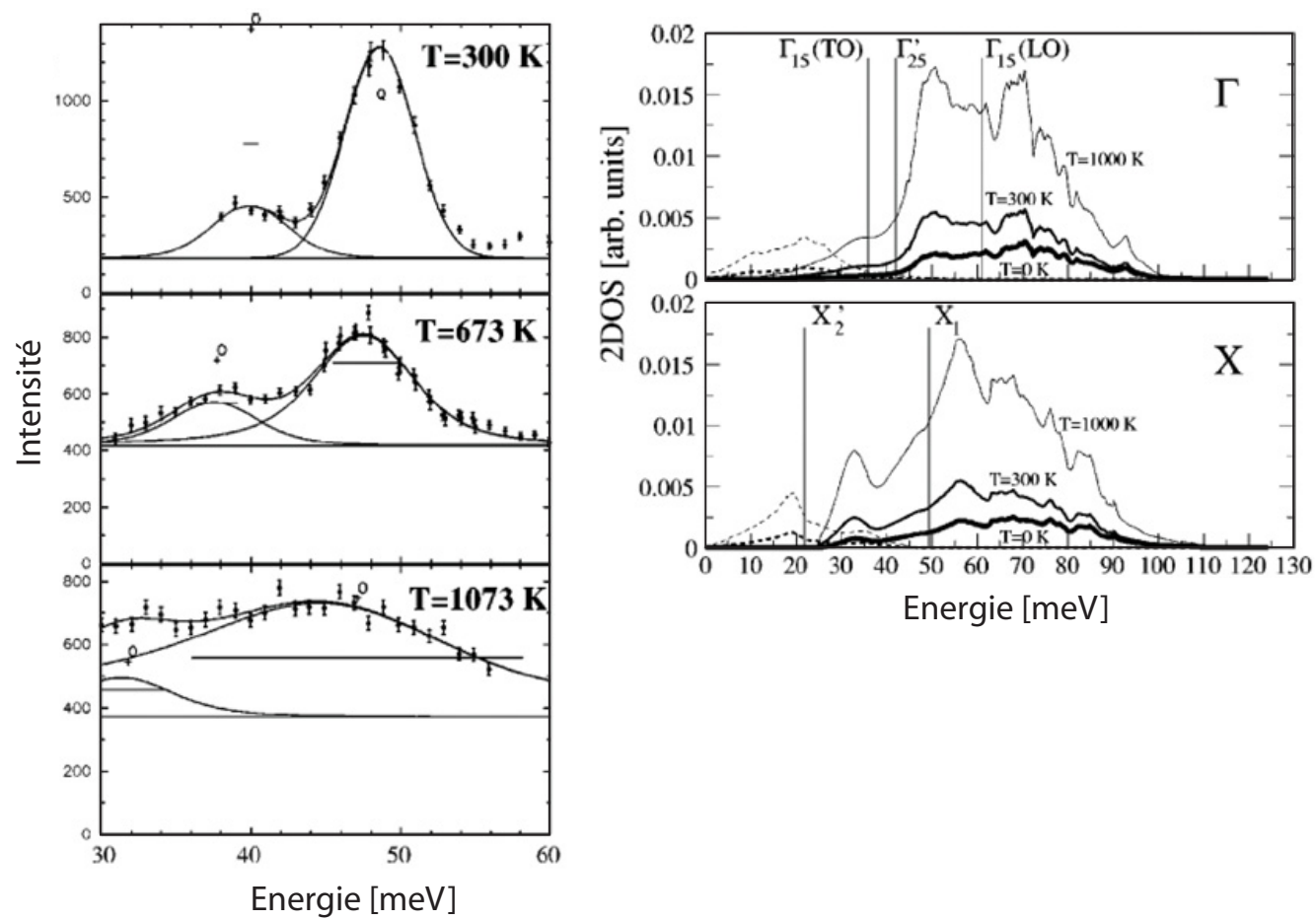

Figure 47. A gauche: élargissement de deux phonons spécifiques du conducteur super-ionique $\mathrm{CaF}_{2}$ en centre de zone. Les mesures ont été faites par diffusion inélastique des neutrons. A $1073 \mathrm{~K}$ la largeur des pics est telle que les deux excitations ne peuvent pratiquement plus être séparées. A droite: densité d'états à deux phonons $D(\vec{k}, \omega)$ pour le point $\Gamma$ et le point $X$. Les courbes en traits pleins correspondent à la contribution des processus de sommation, les courbes en pointillés aux processus de différence. Le rapport entre les largeurs des phonons peut être bien expliqué par la variation de $D(\vec{k}, \omega)$ pour ce qui concerne le point $\Gamma$. Par exemple, l'expérience donne une largeur du mode $\Gamma_{15}(L O)$ trois fois plus élevée que celle du mode $\Gamma_{15}(T O) . D(\vec{k}, \omega)$ peut aussi expliquer qu'à haute température, les largeurs augmentent linéairement avec $T$. La situation n'est pas aussi simple au point $X$. pour le mode $X_{2}^{\prime}$ il y a très peu de volume de phase. Le mode transverse acoustique a une courbure négative (voir figure 31 pour les courbes de dispersion). Cela offre très peu de canaux de désintégration. Il devrait donc posséder une largeur assez faible par rapport aux autres modes. Ce n'est pas ce que nous observons expérimentalement. Les coefficients de couplage pour ce mode doivent, par conséquent, être beaucoup plus forts que la moyenne. La forte anharmonicité dans ce composé serait responsable de la conductivité ionique des ions de fluor à haute température [35].

$\Omega(j, \vec{k})$ est la fréquence et $\Gamma(j, \vec{k})$ la largeur de l'oscillateur $(j, \vec{k})$ (se référer aussi au chapitre III). Nous montrons deux exemples concrets de phonons amortis sur les figures 46 et 47. Dans le premier cas, il s'agit du mouvement de la molécule $\mathrm{N}_{2}$ dans une cage de clathrate. La molécule est petite par rapport à la taille de la cage. Les expériences ont démontré qu'il pouvait y avoir occupation d'une cage par deux molécules hôtes [37]. A haute température, l'amplitude de vibration est suffisamment élevée pour que la molécule de $\mathrm{N}_{2}$ explore les parois répulsives de la cage. Quand la température baisse, l'amplitude diminue et la molécule "se rend compte" du fond plat du potentiel. La fréquence baisse et la largeur augmente. On parle de mode de "rattling" (ou mode hochet, voir chapitre de H. Mutka consacré à ces modes dans cet ouvrage). La susceptibilité mesurée par diffusion inélastique des neutrons se décrit bien par l'expression 4.40 pour l'oscillateur amorti. Les paramètres obtenus pour la fréquence et pour la largeur suivent la dépendance attendue pour un mode mou.

Dans l'exemple suivant nous regardons plus en détail la largeur des phonons dans le conducteur superionic $\mathrm{CaF}_{2}$, déjà abordé dans le contexte de l'expansion thermique. Selon la théorie de perturbation 
[38] seuls les termes d'ordre 3 contribuent à la largeur. Les termes d'ordre quatre n'influent que sur la renormalisation de l'énergie. Formellement on obtient

$$
\begin{aligned}
\Gamma_{(j, \vec{k})}(\omega)= & \frac{18 \pi}{\hbar^{2}} \sum_{j_{1}, \vec{k}_{1}} \sum_{j_{2}, \vec{k}_{2}}\left|V_{3}\left(j, j_{1}, j_{2} \mid \vec{k}, \vec{k}_{1}, \vec{k}_{2}\right)\right|^{2} \\
& \times\left\{\left(n_{1}+n_{2}+1\right)\left[\delta\left(\omega_{1}+\omega_{2}-\omega\right)-\delta\left(\omega_{1}+\omega_{2}+\omega\right)\right]\right. \\
& \left.+\left(n_{2}-n_{1}\right)\left[\delta\left(\omega_{1}-\omega_{2}-\omega\right)-\delta\left(\omega_{1}-\omega_{2}+\omega\right)\right]\right\}
\end{aligned}
$$

où nous avons introduit les abréviations

$$
\omega_{i}=\omega_{j_{i}}\left(\vec{k}_{i}\right) \quad \text { et } \quad n_{i}=\frac{1}{\exp \left(\beta \hbar \omega_{j_{i}}\left(\vec{k}_{i}\right)\right)-1}, \quad i=1,2
$$

pour les fréquences et pour les facteurs d'occupation des modes. Les coefficients anharmoniques $V_{3}\left(j, j_{1}, j_{2} \mid \vec{k}, \vec{k}_{1}, \vec{k}_{2}\right)$ sont difficiles à calculer. En première approximation nous pouvons formuler l'hypothèse selon laquelle ils ne dépendent pas de leurs arguments. ${ }^{90}$ Dans ce cas la largeur $\Gamma_{j, \vec{k}}(\omega)$ est proportionnelle à l'expression

$$
\begin{aligned}
D(\vec{k}, \omega)= & \sum_{j_{1}, \vec{k}_{1}} \sum_{j_{2}, \vec{k}_{2}} \sum_{\vec{G}} \delta\left(\vec{k}-\left(\vec{k}_{1}+\vec{k}_{2}+\vec{G}\right)\right) \\
& \times\left\{\left(n_{1}+n_{2}+1\right) \delta\left(\omega_{1}+\omega_{2}-\omega\right)+\left(n_{2}-n_{1}\right) \delta\left(\omega_{1}-\omega_{2}-\omega\right)\right\} .
\end{aligned}
$$

$\vec{G}$ dénote les vecteurs du réseau réciproque. Cette grandeur est appelée densité d'états à deux phonons. Elle ne dépend que des surfaces de dispersion dans l'approximation harmonique et est par conséquent accessible aux calculs. $D(\vec{k}, \omega)$ mesure le nombre de combinaisons possibles pour que

1. deux phonons $\omega_{1}$ et $\omega_{2}$ fusionnent en un phonon de fréquence $\omega=\omega_{1}+\omega_{2}$ (processus de sommation illustré par le diagramme (a) de la figure 45),

2. un phonon $\omega_{1}$ absorbe un phonon $\omega_{2}$ pour produire un phonon de fréquence $\omega=\omega_{1}-\omega_{2}$ (processus de différence illustré par le diagramme (b) dans la figure 45. Ce processus impliquant la destruction du phonon $\omega_{2}$ est interdit à $T=0$.)

tout en préservant le moment cristallin. En d'autres termes, $D(\vec{k}, \omega)$ mesure le volume de l'espace des phases qui est susceptible de contribuer aux processus anharmoniques pour un mode donné. Si ce volume est très petit pour un ordre de perturbation donné, il pourra s'avérer nécessaire d'avoir recours à des termes de correction d'ordre supérieur. Dans le cas du $\mathrm{CaF}_{2}$, la densité d'états à deux phonons suffit pour expliquer la variation de la largeur des phonons avec la fréquence pour le centre de zone. On a par contre des difficultés avec le mode le plus bas au point $X$. Les constantes anharmoniques des processus contribuant à l'élargissement de ce mode doivent donc être plus importantes que la moyenne.

\subsection{Phonons et transport thermique}

La chaleur dans un solide peut être transportée soit par les électrons soit par les ions. Dans les deux cas, c'est la redistribution de l'énergie cinétique des particules qui est en jeu. Pour le système des ions, l'énergie cinétique est liée directement au niveau d'occupation des phonons. De manière mathématique, le transport de chaleur se décrit par la relation

$$
J_{\alpha}=\sum_{\beta} \kappa_{\alpha \beta} \frac{\partial T}{\partial x_{\beta}}
$$

\footnotetext{
${ }^{90}$ Cette hypothèse sera forcément fausse dans le cas des modes acoustiques, déjà à cause du facteur $1 / \sqrt{8 \omega_{1} \omega_{2} \omega_{3}}$.
} 


\section{$\mathrm{Ge}_{2}$ structure Clathrate de diamant $\mathrm{Ge}_{46}$ vide}
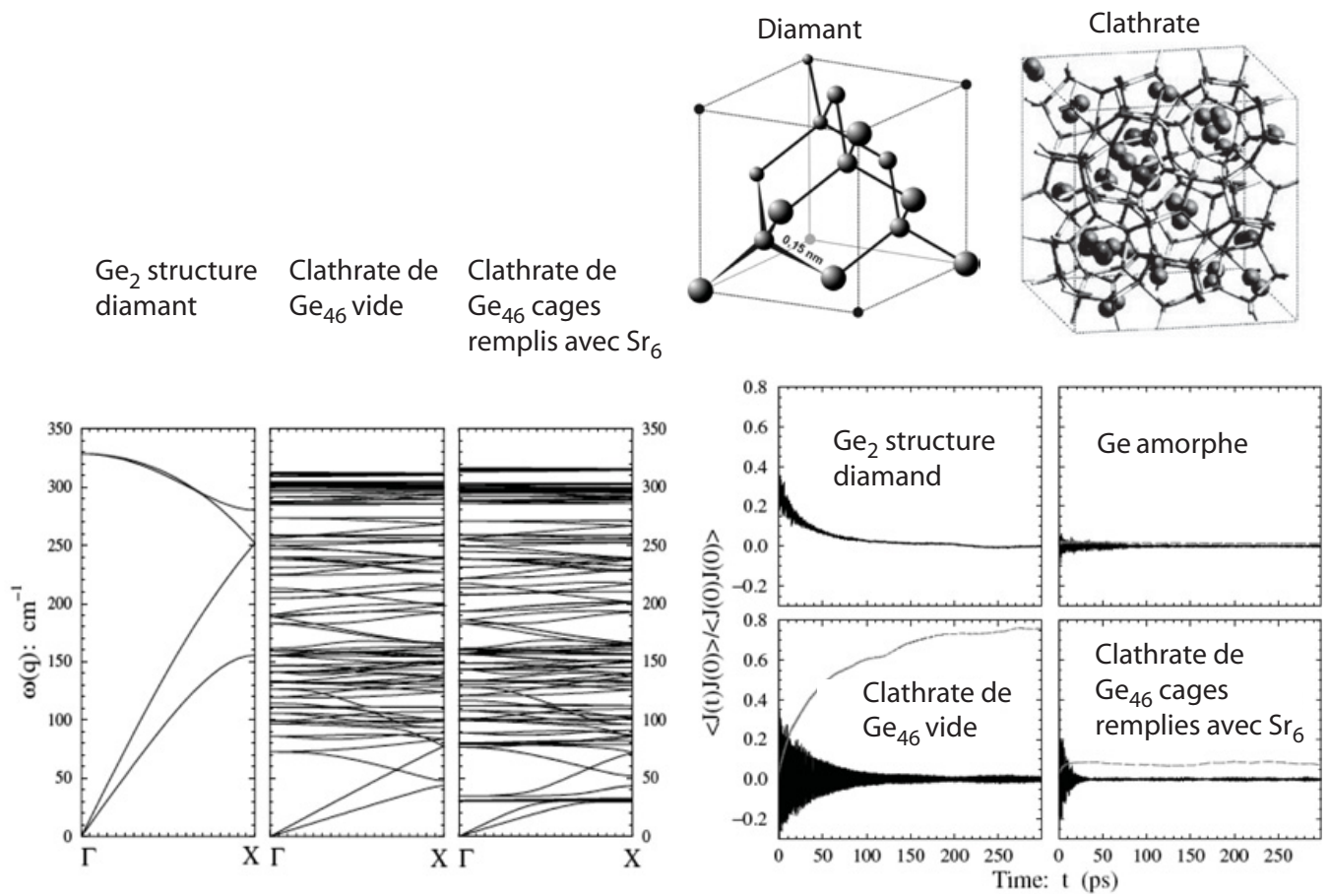

Figure 48. Lois de dispersion et fonctions de corrélation pour le courant thermique $J(t)$ obtenues par des calculs de dynamique moléculaire [13] en utilisant l'expression 2.206. On compare quatre systèmes, qui sont tous basés sur le germanium. Le germanium cristallin possède la structure du diamant avec deux atomes par maille primitive. Les surfaces de dispersion comportent trois modes acoustiques et trois modes optiques. Dans la direction montrée ici, les modes transverses sont dégénérés. Le courant thermique du germanium cristallin décroît de manière exponentielle. Il peut, par conséquent, être décrit en bonne approximation par un seul temps de relaxation $\tau$. Le germanium amorphe est simulé par une grande maille désordonnée de 512 atomes. Le courant thermique chute très rapidement à une valeur proche de zéro. C'est en accord avec l'observation selon laquelle la conductivité thermique du germanium amorphe est extrêmement réduite par rapport au cristal (de l'ordre d'un facteur 100). Le clathrate $\mathrm{Ge}_{46}$ possède 46 atomes par maille primitive. Ce motif de grande taille produit un grand nombre de modes optiques dans une zone de Brillouin réduite par rapport à celle du germanium cristallin. Le courant thermique reflète bien le changement de structure. Il comporte une enveloppe qui n'est pas trop différente de la courbe du cristal simple. Ceci indique que les durées de vie des phonons ne sont pas trop différentes dans les deux composés. Par contre le courant thermique dans le clathrathe oscille fortement autour de zéro. Les fréquences d'oscillations correspondent à la gamme de fréquence des phonons optiques (la composante de forme $\exp \left(-t / \tau_{0}\right)$ n'est responsable que pour $10 \%$ à la fonction de corrélation de $J(t))$. C'est cette composante qui détermine la conductivité thermique, et qui s'avère donc être un facteur 10 en dessous de celle du germanium avec la structure du diamant. Quand on remplit les cages du clathrate avec des atomes de $\mathrm{Sr}$, la forme de la fonction de corrélation du courant thermique reste très similaire à celle trouvée pour le clathrate vide, c'est-à-dire qu'on trouve une enveloppe avec des oscillations fortes. La différence est que l'enveloppe décroît beaucoup plus rapidement en présence des atomes de Sr. Ceci se traduit par une conductivité réduite d'un autre ordre de grandeur. C'est l'influence des modes localisés, qu'on retrouve dans la dispersion comme modes plats autour d'environ $30 \mathrm{~cm}^{-1}$.

qui connecte le flux d'énergie $\vec{J}$ au gradient de température $\vec{\nabla} T$. Les coefficients $\kappa_{\alpha \beta}$ forment le tenseur de la conductivité thermique et relient la composante du gradient de température $\partial T / \partial x_{\beta}$ dans la direction $\beta$ au flux d'énergie $J_{\alpha}$ observé dans la direction $\alpha$.

Nous avons déjà rencontré une version simplifiée de cette relation, qui n'est valable que pour les milieux isotropes, dans l'introduction (équation 2.202 de la section 2.4.2). En appliquant la théorie de la réponse linéaire nous avons également établi une relation entre les fluctuations du flux $\vec{J}$ et les constantes 
de conductivité thermique, que nous rappelons ici

$$
\kappa=\frac{V}{3 k_{\mathrm{B}} T^{2}} \int_{0}^{\infty} \sum_{i=x, y, z}\left\langle J_{i}(t) J_{i}(0)\right\rangle d t
$$

Dans la figure 48, nous montrons des exemples concrets du calcul de ces fonctions de fluctuations. Ils s'agit dans tous les cas de composés de germanium mais avec des structures très différentes. On peut relativement bien corréler les changements structuraux avec les changements dans les fonctions de corrélation du courant thermique, et donc dans la conductivité thermique. On voit en particulier que le nombre ainsi que le type des surfaces de dispersion jouent un rôle important dans la conductivité thermique.

Pour élucider -au moins qualitativement- les mécanismes qui sont responsables de la conductivité thermique, nous allons suivre une approche plus classique que celle des fonctions de corrélations. Elle consiste à décrire le courant thermique par l'intermédiaire de ses porteurs, les phonons, et de faire ensuite un lien avec les constantes de conductivité.

En présence d'un gradient de température, nous obtenons nécessairement un gradient d'énergie interne [1]

$$
\frac{\partial u}{\partial x}=\left(\frac{\partial u}{\partial T}\right)\left(\frac{\partial T}{\partial x}\right)=C(T)\left(\frac{\partial T}{\partial x}\right) .
$$

La dépendance de l'énergie interne par rapport à la température est, comme l'indique cette expression, donnée par la chaleur spécifique. Un phonon donné contribue à la chaleur spécifique à hauteur de (voir 3.184)

$$
C_{j}(\vec{k}, T)=\frac{\left(\hbar \omega_{j}(\vec{k})\right)^{2}}{k_{\mathrm{B}} T^{2}} n_{j}(\vec{k})\left(n_{j}(\vec{k})+1\right)=\frac{\left(\hbar \omega_{j}(\vec{k})\right)^{2}}{2 k_{\mathrm{B}} T^{2}} \frac{1}{\sinh ^{2}\left(\frac{\hbar \omega_{j}(\vec{k})}{2 k_{\mathrm{B}} T}\right)} .
$$

La théorie cinétique du transport est basée sur l'hypothèse selon laquelle le courant est maintenu non perturbé sur une distance $l$ pour un temps $\tau$. $l$ est le libre parcours moyen et $\tau=l / v$ le temps de collision, respectivement. La théorie cinétique donne pour le tenseur de conductivité

$$
\kappa_{\alpha \beta}(T)=\sum_{j} \sum_{\vec{k}}\left[C_{j}(\vec{k}, T) \cdot v_{j \alpha}(\vec{k}, T) \cdot v_{j \beta}(\vec{k}, T) \cdot \tau_{j \beta}(T)\right],
$$

où $v_{j \alpha}(\vec{q}, T)$ sont les vitesses de groupe, c'est-à-dire les dérivées des surfaces de dispersion de type $j$ par rapport à la direction $\alpha$.

Dans l'approximation harmonique, il est possible de déterminer les vitesses de groupe des phonons. Les temps de relaxation relèvent du domaine des interactions anharmoniques. Il est relativement difficile de les déterminer pour des systèmes réels. Pour cette raison, le sujet de la conductivité thermique reste d'une grande actualité et donne souvent lieu à des discussions controversées, en particulier quand la conductivité thermique observée est fortement réduite par rapport à des systèmes de référence. C'est par exemple le cas des amorphes par rapport aux cristaux ainsi que des systèmes avec cages par rapport à des homologues sans cages. Quel aspect des vibrations est responsable pour ces différences? Il ne peut certainement pas être le but de cette section de répondre à ces questions. Nous nous limiterons à quelques remarques assez générales.

Nous commençons par constater que chaque phonon porte une énergie $\hbar \omega$ avec sa vitesse de groupe. Le courant thermique associé à un groupe de phonons avec des nombres d'occupation $n_{j}(\vec{k})$ est donc donné par l'expression

$$
\vec{J}=\frac{1}{V} \sum_{\vec{k}, j} \hbar \omega_{j}(\vec{k}) \vec{\nabla}_{\vec{k}}\left(\omega_{j}(\vec{k})\right) n_{j}(\vec{k})
$$


Les porteurs de chaleur les plus efficaces sont les phonons avec une grande vitesse de groupe. Les modes correspondant à ce critère sont en géneral les phonons acoustiques ainsi que les modes qui, même s'ils font nominalement partie des branches optiques, dans les faits prolongent les modes acoustiques. Le croisement de branches optiques avec des branches acoustiques aura en général tendance à diminuer la vitesse de groupe moyenne des porteurs de chaleur. Des structures avec des grandes mailles (et/ou contenant un grand nombre de motifs par maille) auront donc toutes les chances de posséder une conductivité thermique réduite. Dans ce contexte, la maille doit être considerée comme le volume dans lequel règne le désordre, c'est-à-dire dans lequel il n'y a pas de symétrie de translation. Dans le cas extrême, la maille coïncide avec le cristal. Nous retrouvons l'amorphe qui comme nous l'avons déjà indiqué possède une conductivité thermique réduite par rapport au cristal.

A l'équilibre, il y a autant de phonons avec une vitesse de groupe positive qu'avec une vitesse de groupe négative. C'est une conséquence directe de la propriété $\omega_{j}(\vec{k})=\omega_{j}(-\vec{k})$ des relations de dispersion qui implique que $n_{j}(\vec{k})=n_{j}(-\vec{k})$. Le courant thermique total à l'équilibre est donc nul car $\vec{\nabla}_{\vec{k}}\left(\omega_{j}(-\vec{k})\right)=-\vec{\nabla}_{\vec{k}}\left(\omega_{j}(\vec{k})\right)$, rendant nulle la somme dans l'éq. 4.49. Ce résultat est attendu pour un système à l'équilibre. Pour initier un courant thermique non nul, il faut donc créer une distribution de phonons déséquilibrée par rapport au vecteur d'onde $\vec{k}$

$$
\sum_{j, \vec{k}} \vec{k} n_{j}(\vec{k}) \neq 0 .
$$

Dans un cristal harmonique il n'y a aucune interaction entre les phonons. Par conséquent le moment cristallin sera conservé et un courant thermique, une fois créé, sera maintenu en dépit de l'absence de tout gradient de température. Un cristal harmonique a donc une conductivité thermique infinie. C'est déjà moins évident.

Si maintenant on tient compte des interactions anharmoniques entre phonons, deux cas sont à distinguer :

1. Les interactions entre phonons ne comportent que des processus normaux. Des processus normaux sont des processus qui conservent strictement la somme des moments cristallins. Par exemple pour un processus normal de fusion à trois phonons (voir figure 45(a) et figure 49), on vérifiera

$$
\vec{k}_{1}+\vec{k}_{2}=\vec{k}_{3}
$$

Des processus normaux seront incapables de ramener la distribution des phonons complètement à l'équilibre. C'est un constat qu'on peut démontrer strictement, mais qui est déjà intuitivement assez clair. On trouve le même phénomène dans un nuage de gaz en mouvement. Les molécules du gaz vont se heurter les unes aux autres, mais lors de chaque collision l'impulsion est préservée. Le centre de gravité du nuage continuera à se déplacer avec son impulsion initale. En parfaite analogie avec le nuage de gaz, les facteurs d'occupation des phonons vont converger vers une distribution qui conservera la somme initiale des moments cristallins. Le courant thermique est maintenu, la conductivité est donc infinie comme dans le cas d'un système harmonique.

2. On parle de processus "Umklapp = (renversement de la direction)" quand le moment cristallin n'est conservé qu'à un vecteur du réseau réciproque près (voir figure 49). Pour un processus Umklapp de fusion à trois phonons, on vérifiera donc

$$
\vec{k}_{1}+\vec{k}_{2}=\vec{k}_{3}+\vec{G}
$$

Les processus Umklapp sont capables de renverser la vitesse de groupe. Ils sont donc capables de rétablir l'équilibre de la distribution des phonons et amènent à une conductivité thermique finie. Une 


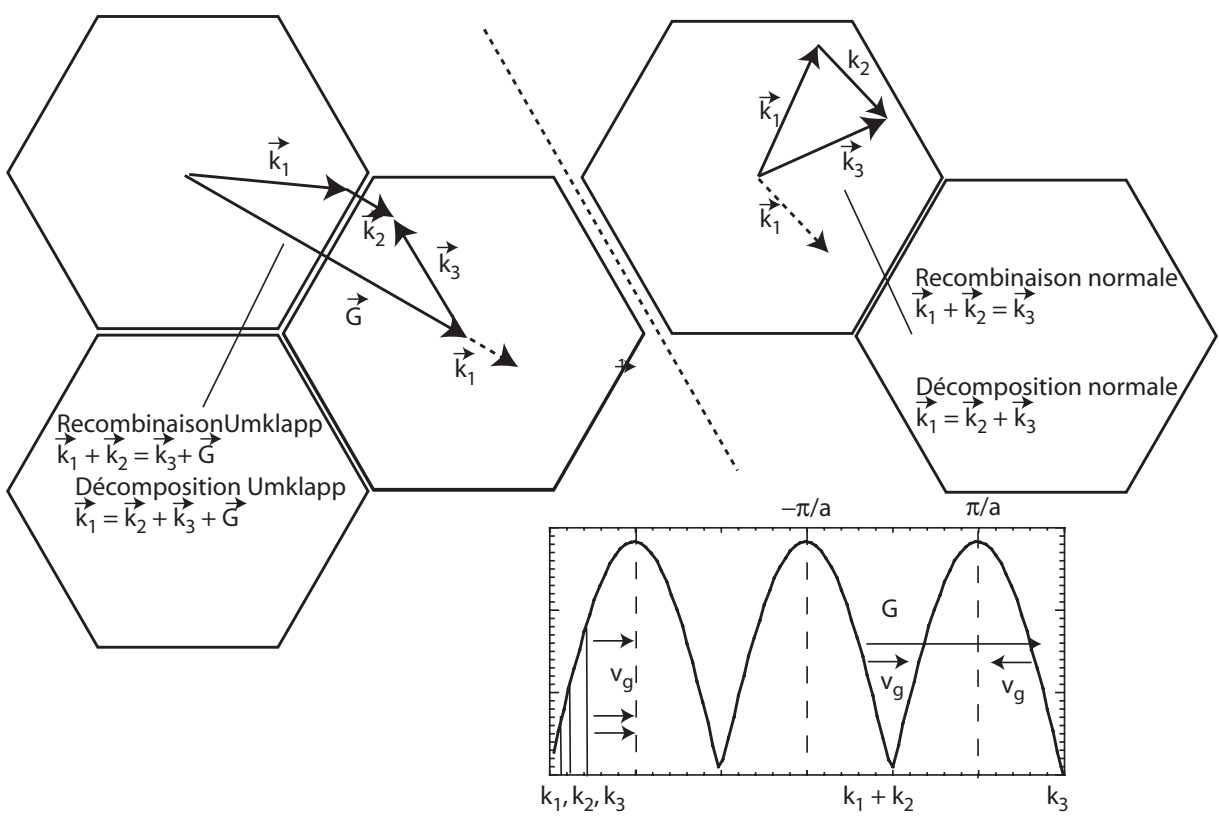

Figure 49. Processus Umklapp et processus normaux. Nous ne montrons que des illustrations pour des processus à trois phonons. Dans une recombinaison normale, deux phonons avec des vecteurs d'onde $\vec{k}_{1}$ et $\vec{k}_{2}$ se combinent pour donner un phonon avec un vecteur $\vec{k}_{3}$, naturellement en conservant l'énergie. Les vecteurs $\vec{k}_{1}, \vec{k}_{2}$ et $\vec{k}_{3}$ sont à prendre dans la première zone de Brillouin qui, dans cette illustration, est de forme hexagonale. Un processus de décomposition normale suit le même schéma. Seul le rôle des phonons est interchangé. Un phonon avec un vecteur d'onde $\vec{k}_{1}$ se décompose en deux phonons avec des vecteurs d'ondes $\vec{k}_{2}$ et $\vec{k}_{3}$. Quand il s'agit d'un processus de recombinaison Umklapp, la somme des vecteurs d'onde $\vec{k}_{1}$ et $\vec{k}_{2}$ sort de la première zone de Brillouin et idem pour la somme de $\vec{k}_{2}$ et $\vec{k}_{3}$ dans le cas de la décomposition. Comme nous essayons de l'illustrer dans le petit schéma de courbe de dispersion en bas à droite, les vitesses de groupe ne peuvent être inversées que par des processus d'Umklapp. Ces processus sont donc capables d'inverser la direction du courant d'énergie. C'est la raison pour laquelle ce sont les processus Umklapp qui déterminent la conductivité thermique d'un gaz de phonons. Pour que deux phonons puissent se combiner en un, il faut à la fois conserver le moment cristallin et l'énergie. Pour les phonons d'une branche acoustique, ce n'est pas possible sauf si la dispersion est rigoureusement linéaire. Le processus montré à titre d' illustration dans le schéma de la courbe de dispersion est par conséquent interdit parce que dans la réalité la courbe de dispersion possède une courbure négative. Nous voudrions terminer avec une remarque concernant la classification des processus en processus normaux et en processus Umklapp. A priori rien ne nous oblige à choisir la zone de Brillouin pour paver l'espace réciproque. En changeant de maille primitive on pourrait donc convertir des processus normaux en processus Umklapp. En d'autres termes la définition d'un processus Umklapp peut paraître ambigüe. Nous affirmons que ce changement de maille ne changerait en rien la physique mais rendrait la discussion des processus anharmoniques très lourde. Ce qui compte pour la conductivité thermique, c'est l'inversion de la vitesse de groupe. Le choix de la zone de Brillouin pour décrire cette inversion est idéal parce qu'il s'agit d'une zone de proximité. En d'autres termes, la zone de Brillouin regroupe tous les vecteurs d'onde autour des vecteurs du réseau réciproque les plus proches. La puissance de ce concept de proximité devient évidente quand on essaye de démontrer que lors d'une décomposition, la vitesse de groupe ne pourra pas augmenter, ni pour les processus normaux ni pour les processus Umklapp [47].

expression analytique [46] peut être obtenue pour le temps de relaxation résultant de la désintegration d'un phonon en deux autres par processus Umklapp sous certaines conditions. ${ }^{91}$ Au premier ordre des perturbations et en se limitant à des processus qui n'impliquent qu'une seule branche de dispersion,

\footnotetext{
91 Le sujet nous semble trop complexe pour être détaillé ici. Le lecteur est prié de se renseigner lui-même sur le champs d'application de cette théorie en consultant le papier original. Nous la mentionnons ici pour donner une idée des grandeurs physiques qui entrent dans la détermination des temps de relaxation.
} 
on obtient pour un mode $j$ de vecteur d'onde $\vec{k}$

$$
\frac{1}{\tau_{j}(\vec{k})}=\sum_{\vec{q}} 2\left|C_{3}\right|^{2} \frac{\hbar}{M^{3} \omega \omega_{1} \omega_{2}} \pi \delta\left(\omega+\omega_{1}-\omega_{2}\right)\left(n\left(\omega_{1}\right)-n\left(\omega_{2}\right)\right)
$$

où le coefficient de couplage s'exprime selon

$$
\left|C_{3}\right|^{2}=\frac{4 \gamma_{j}^{2} V_{\text {prim }}}{r} \frac{M^{2}}{v_{g}^{2}} \omega^{2} \omega_{1}^{2} \omega_{2}^{2} .
$$

On s'est limité à un système avec une seule masse $M . V_{\text {prim }}$ est le volume de la maille primitive et $r$ le nombre d'atomes dans cette maille. $\gamma_{j}$ est le paramètre de Grüneisen associé au mode en question, qu'on a déjà rencontré lors de la discussion de l'expansion thermique dans la section 4.1. La sommation court sur tous les processus de désintégration qui respectent les relations de conservation du moment cristallin

$$
\vec{k}+\vec{q}=\vec{q}^{\prime}+\vec{G}
$$

et de l'énergie

$$
\omega_{j^{\prime \prime}}\left(\vec{q}^{\prime}+\vec{G}\right)=\omega_{j^{\prime \prime}}\left(\vec{q}^{\prime}\right)=\omega_{j}(\vec{k})+\omega_{j^{\prime}}(\vec{q}) .
$$

Le temps de relaxation $\tau_{j}(\vec{k})$ est le temps qu'il faudra au système pour revenir à l'équilibre, si seulement l'occupation du mode $\omega_{j}(\vec{k})$ en départait. Le fait que lors de la désintegration, l'énergie et le moment cristallin doivent être préservés est une restriction sévère qui a des conséquences immédiates. Cela implique par exemple qu'un phonon avec une dispersion linéaire $\omega=c k$ ne pourra se désintégrer en deux autres phonons de ce type que si ces phonons possèdent des vitesses de groupe plus basses [47]. Ainsi un phonon longitudinal pourra se désintégrer en deux phonons transverses

$$
L \rightarrow T+T
$$

ou en un phonon transverse plus un phonon longitudinal

$$
L \rightarrow L+T,
$$

mais pas en deux phonons longitudinaux sauf si ceux-ci possédaient une dispersion anormale. Puisque les phonons transverses fournissent en général la surface de dispersion la plus basse, ils ne peuvent pas se désintégrer par des processus Umklapp. Ils ont donc des durées de vie assez longues à basse température.

Seuls les phonons de fréquence $\hbar \omega<k_{\mathrm{B}} T$ sont peuplés. A très basse température, cela nous limite aux modes acoustiques avec un vecteur d'onde très petit. La combinaison de tels vecteurs d'onde donnera impérativement lieu à des processus normaux. Pour obtenir un processus Umklapp, il faudra rajouter un phonon du bord de la zone de Brillouin dont l'occupation diminue de manière exponentielle avec la température. L'effet des processus Umklapp est ainsi prépondérant à une température relativement haute. A basse température, la conductivité thermique est régie par la chaleur spécifique (voir éq. 4.48) qui augmente comme $T^{3}$ (voir expression 3.184). Dans un domaine de température intermédiaire, ce sont des processus qui impliquent la présence de désordre (par exemple isotopique) et/ou l'effet de taille finie des cristaux réels qui sont les facteurs limitants les plus important du libre parcours et donc de la conductivité.

Nous montrons un exemple de calcul selon l'expression 4.53 et sa comparaison avec l'expérience sur la figure 50.

En dehors de cette compréhension générale, réussir à calculer la conductivité thermique à partir de la dynamique du réseau promet d'être d'une grande utilité pour optimiser des circuits miniaturisés. Dans tous ces dispositifs il faut dégager la chaleur produite par les courants électriques en utilisant des ponts 

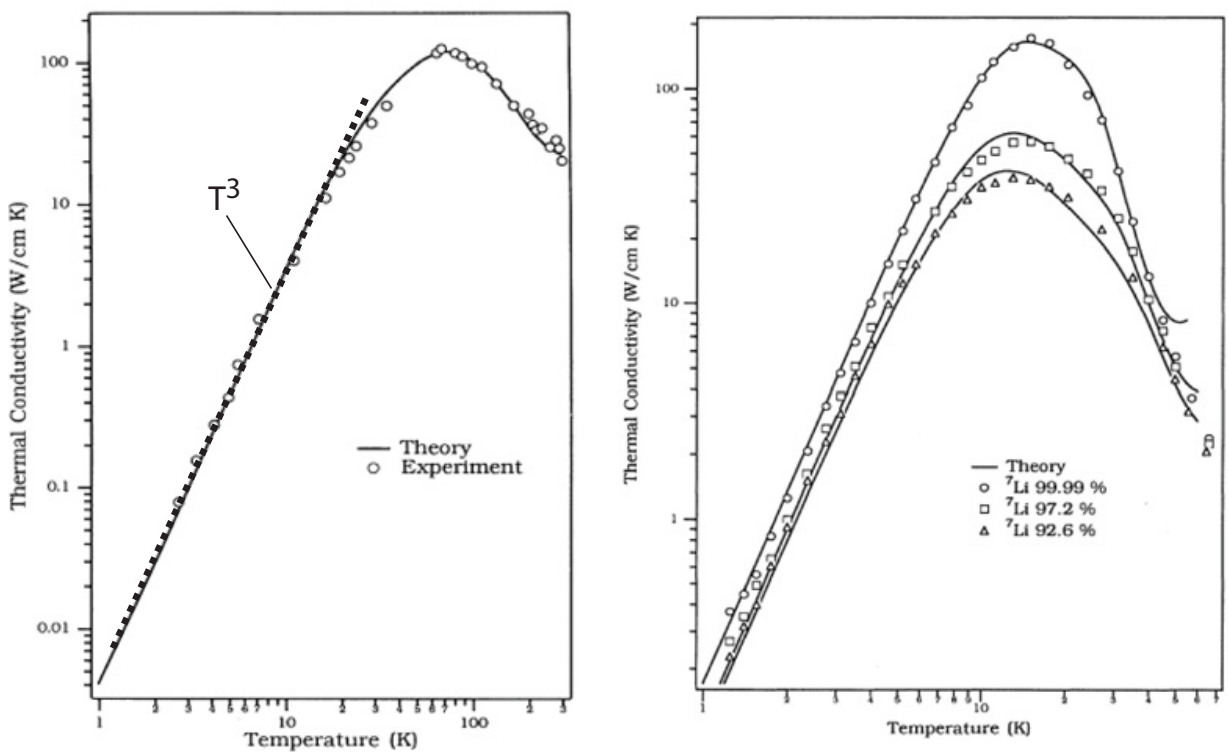

Figure 50. Conductivité thermique pour le diamant et pour le LiF calculée dans un formalisme où le temps de relaxation est basé sur l'expression 4.53 [46]. Les calculs incluent aussi des termes qui prennent en compte la diffusion des phonons par des défauts, ainsi que l'effet de la taille finie des cristaux. Les paramètres de Grüneisen sont des paramètres libres et adaptés aux expériences. La conductivité thermique augmente à basse température, comme la chaleur spécifique, c'est à dire en $T^{3}$. Ceci est dû au peuplement des porteurs de chaleur. Dans les cristaux très purs, l'influence de la taille est bien marquée. Les expériences pour le LiF à $99.99 \%$ de pureté isotopique ont été obtenus avec des cristaux de dimension linéaire d'à peu près $10 \mathrm{~mm}$. En diminuant la taille des cristaux d'un ordre de grandeur, on perd un ordre de grandeur dans la conductivité thermique à basse température. Dans le cas du LiF, ont voit également très bien l'effet dû au désordre isotopique. Moins de $10 \%$ de défauts réduisent la conductivité de pratiquement un ordre de grandeur au maximum de la courbe. L'effet de la taille et de la pureté isotopique indique la complexité du problème quand on est confronté à étudier la conductivité thermique. Du côté expérimental, la qualité des échantillons joue un rôle fondamental. Le problème devient très prononcé quand les systèmes étudiés, comme par exemple les clathrates, ont par nature la tendance à former des structures plus ou moins poreuses [14] (voir figure 12). Du côté de la théorie, on réalise que pour expliquer la conductivité thermique il est indispensable d'utiliser des descriptions détaillées du système. Dans la plupart des cas on n'observe pas de grosses anomalies dûes à des anharmonicités énormes mais plutôt les conséquences subtiles de l'anharmonicité normale. A haute température, les processus Umklapp se mettent en place tandis que la chaleur spécifique reste plus ou moins constante. De ces deux effets combinés resulte une baisse de la conductivité thermique. Les calculs montrent que les phonons acoustiques doivent d'abord se combiner par les processus normaux avant de participer à des processus Umklapp impliquant des phonons de bord de zone.

thermiques, qui ont eux aussi les dimensions du nanomètre. On peut s'imaginer des nanofils en silicium ou des nanotubes de carbone. Les propriétés de conduction sont fortement modifiées quand on passe du volume à la taille finie $[48,49]$ (voir la figure 51 ).

Non seulement le spectre des phonons d'un nanotube isolé diffère considérablement de celui d'un feuillet de graphène, en dépit du fait que les nanotubes peuvent être considérés comme de simples feuillets de graphène enroulés (voir figure 52, et le chapitre consacré à ce sujet dans cet ouvrage), mais en plus les canaux de recombinaison et de décomposition dûs aux effets anharmoniques, sont modifiés. En particulier, la notion de vecteur de propagation doit être adaptée au caractère unidimensionnel du nanotube. Sa composante perpendiculaire à l'axe du tube ne peut plus être considerée comme quasicontinue. Elle prend des valeurs discrètes

$$
\vec{k}_{\perp}=n_{\perp} \frac{2 \pi}{\left|C_{h}\right|^{2}} \vec{C}_{h}, \quad n_{\perp}=-r / 2, \ldots, 0,1, \ldots, r / 2,
$$



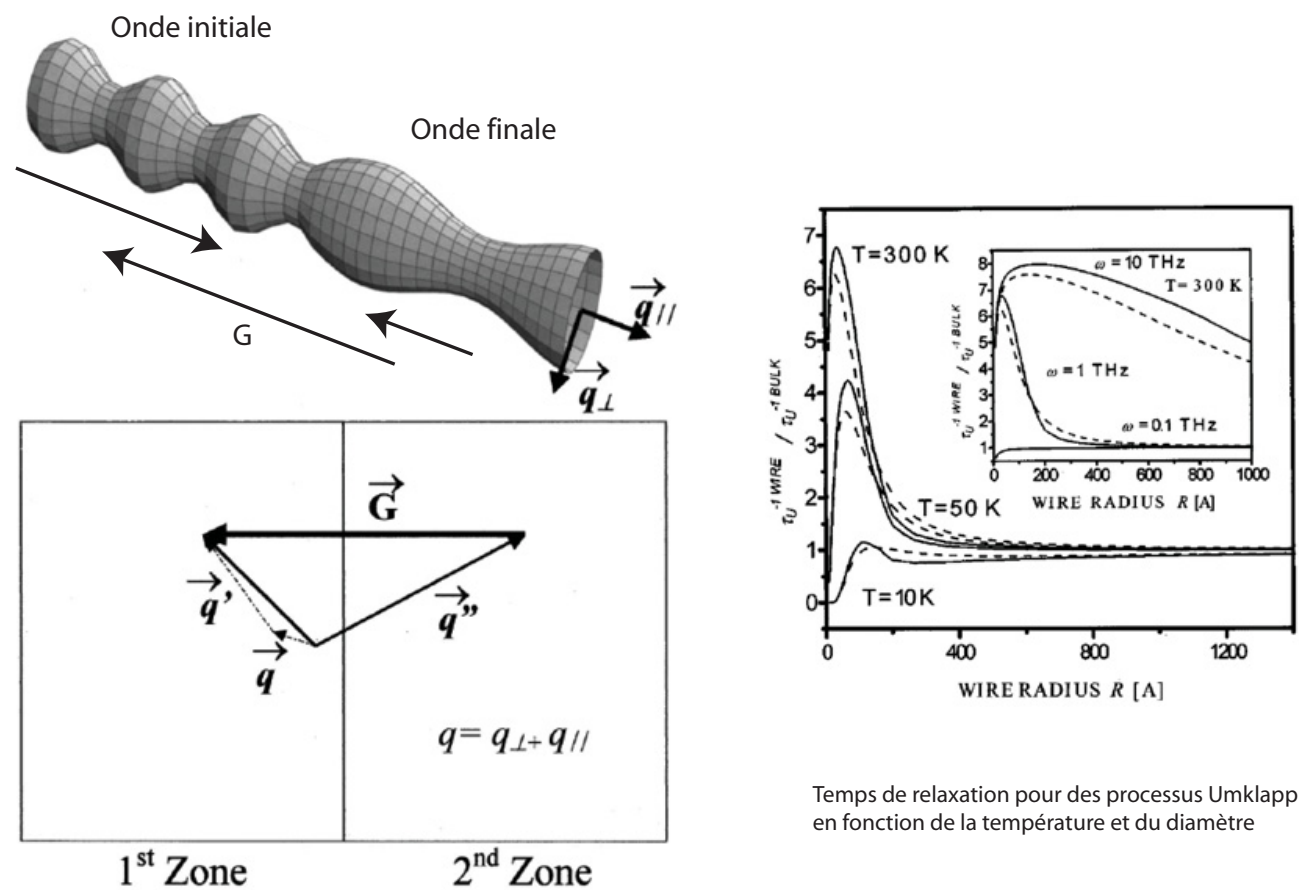

Figure 51. Dans le cas d'un nanofil, les processus Umklapp peuvent se visualiser dans l'espace direct au moins pour les modes élastiques. Cette visualisation possède à nos yeux une certaine valeur pédagogique. Le schéma est pris de l'article de Khituna et Wang [48]. Un mode élastique (phonon acoustique) avec un vecteur d'onde $\vec{q}^{\prime \prime}$ se décompose en deux modes avec vecteurs d'ondes $\vec{q}$ et $\vec{q}^{\prime}$. S'agissant d'un processus Umklapp, la composante axiale, c'est-à-dire le long du fil, est preservée à un vecteur réciproque $\vec{G}$ près. La direction de propagation est inversée et la longueur de modulation a augmenté dû au fait que les vecteurs d'ondes les régissant sont devenus plus courts. L'énergie doit naturellement être conservée, c'est-à-dire $\omega\left(\vec{q}^{\prime \prime}\right)=\omega(\vec{q})+\omega\left(\vec{q}^{\prime}\right)$. Attention à ne pas prêter trop de réalité à cette représentation graphique, qui restera un pur outil pédagogique. Les états, et en particulier l'état quantique correspondant à la superposition des deux phonons finaux, ne sont pas des états cohérents et surtout pas des ondes classiques. Ce point a déjà été élaboré dans le texte. La figure de droite montre le taux de relaxation en fonction de la température et du diamètre d'un nanofil de silicium. Les valeurs pour le silicium en volume sont pris comme référence. On voit que l'effet de taille devient prononcé à partir d'une dizaine de nanomètres.

avec $r$ le nombre de paires d'atomes dans la cellule primitive du tube et $\vec{C}_{h}$ le vecteur chiral du tube. Cette limitation modifie considérablement le nombre et le type de combinaisons de phonons capables de subir des processus de Umklapp. La conservation du moment cristallin discret perpendiculaire à l'axe du tube correspond à la conservation du moment angulaire. Seul les processus anharmoniques, qui conservent le moment angulaire, sont permis.Les restrictions pour les processus de Umklapp deviennent de plus en plus sévères à fur et à mesure que le diamètre du tube diminue. On s'attend donc à une augmentation significative de la conductivité thermique. Pour des tubes de petit diamètre et sans défaut, des $\kappa$ de plusieurs $\mathrm{kW} /(\mathrm{m}-\mathrm{K})$ semblent possibles. Les calculs convergent sur ce point. Il faut néanmoins souligner que les valeurs absolues calculées par plusieurs groupes montrent une variation d'au moins un ordre de grandeur ${ }^{92}$ Ces difficultés -en dépit de la bonne connaissance de la dynamique des nanotubessoulignent une fois de plus que le calcul de la conductivité thermique continue à constituer un défi majeur.

92 Voir la discussion dans l'article de Gu et Chen [49] pour plus de détails. 


\subsection{Couplage électron-phonon}

De même qu'un cristal harmonique sans défauts possède une conductivité thermique infinie, les électrons de Bloch sont des conducteurs parfaits. Ce constat est la conséquence du fait que des paquets d'onde construits à partir d'une bande d'électrons de Bloch autour d'un vecteur d'onde $\vec{k}_{0}$ (voir section 2.1.4 et 3.13) se propageront avec une vitesse moyenne de

$$
\vec{v}_{j}\left(\vec{k}_{0}\right)=\left.\frac{1}{\hbar} \vec{\nabla}_{\vec{k}} E_{\mathrm{el}}^{j}(\vec{k})\right|_{\vec{k}_{0}},
$$

qui ne dépendra pas du temps. Il est évident que ce mouvement électronique sans dissipation d'énergie ne correspond pas à l'expérience. Les métaux ont une résistance finie, qui de plus dépend fortement de la température. La raison qui explique l'incapacité du modèle de Bloch à rendre compte de cette réalité, tient au fait qu'il ne décrit pas les interactions entre électrons, ni celles entre ions et électrons. Nous manquons donc des aspects essentiels de la physique en appliquant ce modèle trop simplifié.

Dans ce chapitre, qui ne couvre que les aspects de la physique des solides liés aux excitations du réseau, nous nous limiterons à discuter les interactions des électrons avec le réseau. Nous maintiendrons le concept d'électrons indépendants et n'abandonnerons uniquement la notion du réseau rigide inhérente à l'expression 3.255. Les états de Bloch étant déduits dans l'hypothèse d'un réseau parfaitement périodique, on comprend que les vibrations qui détruisent cette périodicité, constitueront des canaux de dissipation.

En traitant l'exemple des fullerènes, nous avions vu que les vibrations des molécules $\mathrm{C}_{60}$ réagissent fortement à un transfert de charge. Cet effet se comprend par la modification des interactions entre atomes de carbone induite par la présence des électrons. On peut inverser l'argument et supposer que l'occupation d'un phonon rendra la molécule de $\mathrm{C}_{60}$ plus ou moins attirante pour les électrons. Un électron plus ou moins localisé, qui saute d'une molécule de $\mathrm{C}_{60}$ à une autre dans un cristal métallique de fullérène, a toutes les chances d'inciter celle-ci à vibrer sur son passage. Les vibrations seront encore présentes alors que l'électron sera depuis longtemps parti à cause de la faiblesse relative de sa masse qui impose une échelle de temps typique des électrons très petite. Ces vibrations auront une influence sur les autres électrons du voisinage. La vibration de la molécule transmet donc une interaction indirecte entre les électrons. Ce phénomène est connu sous le nom de couplage électron-électron par médiation de phonon. Si cette interaction est attractive, elle peut déclencher la formation de paires d'électrons, appelées "paires de Cooper". La condensation de ces paires sera responsable de la supraconductivité. L'interaction des électrons avec le réseau peut donc être rendue responsable à la fois de la résistance et de l'absence de résistance dans les métaux. Actuellement les pires conducteurs à température élevée se révèlent souvent les meilleurs supraconducteurs à basse température.

L'interaction entre les ions et les électrons ne peut alors pas être décrite par un simple potentiel électronique ressenti par les ions et vice versa. On sort du cadre de l'approximation adiabatique introduite dans la section 3.1. Elle avait déjà été identifiée à ce moment comme problématique pour les métaux en raison de l'absence d'une séparation nette des échelles d'énergies. Néanmoins nous pouvons utiliser les concepts developpés à l'aide de l'approximation adiabatique, c'est-à dire les phonons et les électrons de bandes, comme points de départ de l'étude des interactions entre électrons et réseau. Dans l'hypothèse où le couplage électron-phonon n'est pas trop fort, cela nous permet de traiter ce dernier par la théorie des perturbations. Nous partons donc de deux systèmes dont nous sommes capables de décrire les états quantiques. Le premier système est celui des phonons harmoniques maintenant bien connu dont l'Hamiltonien est

$$
\mathbf{H}=\sum_{\vec{k}} \sum_{j=1}^{3 r} \hbar \omega_{j}(\vec{k})\left(\mathbf{a}_{j}^{+}(\vec{k}) \mathbf{a}_{j}(\vec{k})+\frac{1}{2}\right) .
$$




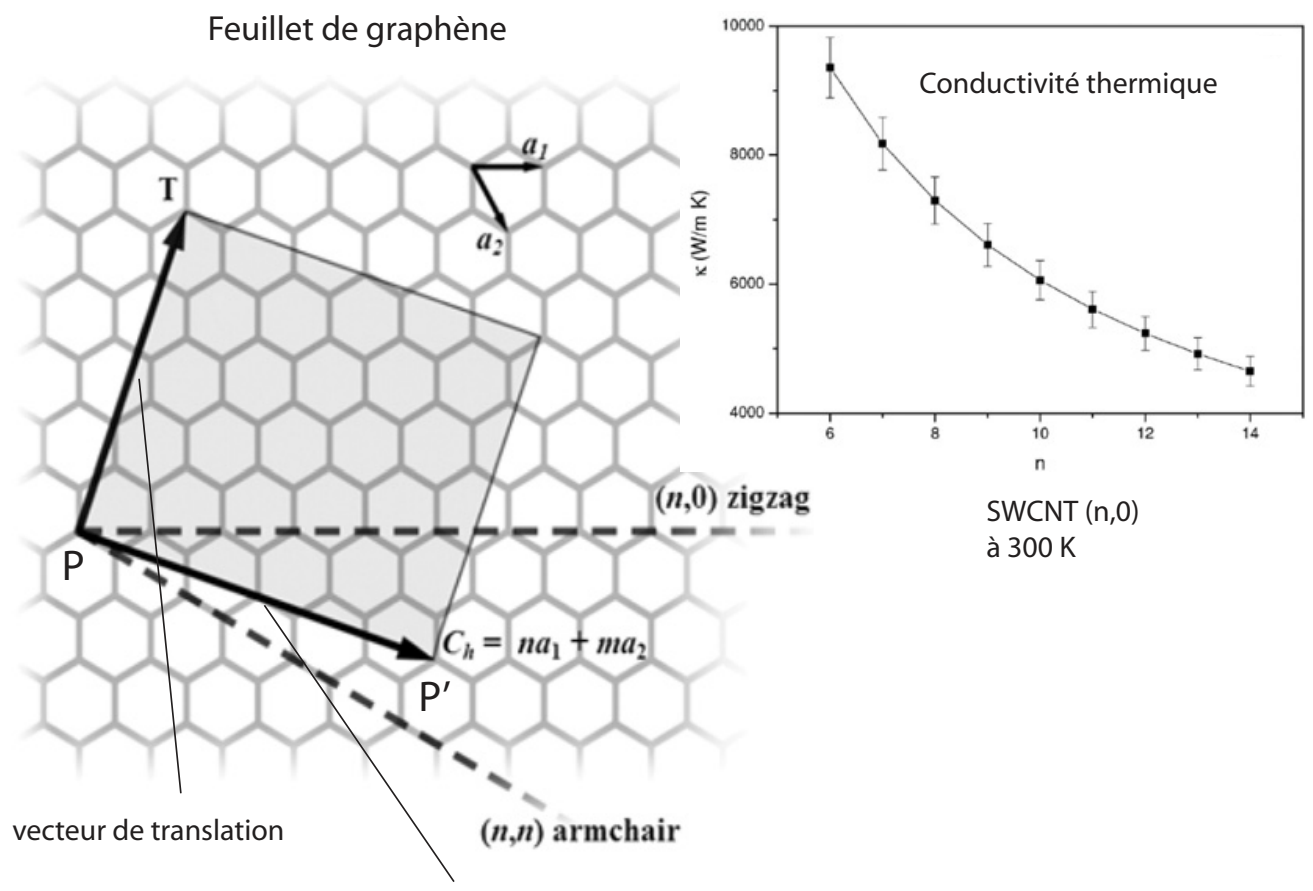

vecteur chiral

Figure 52. Un nanotube de carbone peut être consideré comme un feuillet de graphène bi-dimensionnel enroulé sur lui-même. Le vecteur $\vec{C}_{h}$, appelé vecteur chiral, permet de définir la géométrie d'un nanotube de manière univoque. Ce vecteur fait correspondre les points $P$ et $P^{\prime}$ après enroulement de la bande de graphène et définit ainsi la circonférence du tube. La composante du vecteur d'onde $\vec{k}$ le long de $\vec{C}_{h}$ dans le système du feuillet se transforme en vecteur $k_{\perp}$ lorsque le tube est enroulé. Les conditions de périodicité induites par l'enroulement rendent les valeurs de $\vec{k}_{\perp}$ discrètes (voir équation 4.59). La figure de droite montre les résultats d'un calcul pour des nanotubes de type zig-zag $(n, 0)$ [50]. On découvre une nette dépendance de la conductivité du diamètre des tubes. Les valeurs absolues indiquées ici sont à prendre avec précaution. Les prédictions, ainsi que les mesures, varient considérablement sur ce point.

Le deuxième système est celui des électrons indépendants ${ }^{93}$ avec l'Hamiltonien

$$
\mathbf{H}=\sum_{\vec{q}} \sum_{m, \sigma} \epsilon_{m, \sigma}(\vec{q})\left(\mathbf{c}_{m, \sigma}^{+}(\vec{q}) \mathbf{c}_{m, \sigma}(\vec{q})\right),
$$

où les indices $m$ et $\sigma$ désignent la bande et le spin, respectivement. Le vecteur d'onde $\vec{q}$ court, comme pour les phonons, sur toute la zone de Brillouin.

Nous introduisons un couplage entre les deux systèmes par l'intermédaire d'un terme

$$
\begin{aligned}
& \frac{1}{\omega_{j}(\vec{k})} \sum_{\vec{k}, j} \sum_{\vec{q}} \sum_{m, n} \sum_{\sigma, \sigma^{\prime}} g\left(\vec{q}, m, n, \sigma, \sigma^{\prime} \mid \vec{k}, j\right) \\
& \quad \times\left[\mathbf{c}_{m, \sigma}^{+}(\vec{k}+\vec{q}) \mathbf{c}_{n, \sigma^{\prime}}(\vec{q}) \mathbf{a}_{j}(\vec{k})+\mathbf{c}_{m, \sigma}^{+}(\vec{k}+\vec{q}) \mathbf{c}_{n, \sigma^{\prime}}(\vec{q}) \mathbf{a}_{j}^{+}(-\vec{k})\right] .
\end{aligned}
$$

\footnotetext{
93 Il peut s'agir des électrons de Bloch, des électrons de Hartree-Fock ou des électrons issus des équations de Kohn et Sham [44] basées sur la théorie de la fonctionnelle de la densité [45]. L'important est qu'on arrive à traiter l'interaction entre électrons de manière effective, c'est-à-dire en l'incluant dans un potentiel effectif $V_{\text {eff }}$.
} 


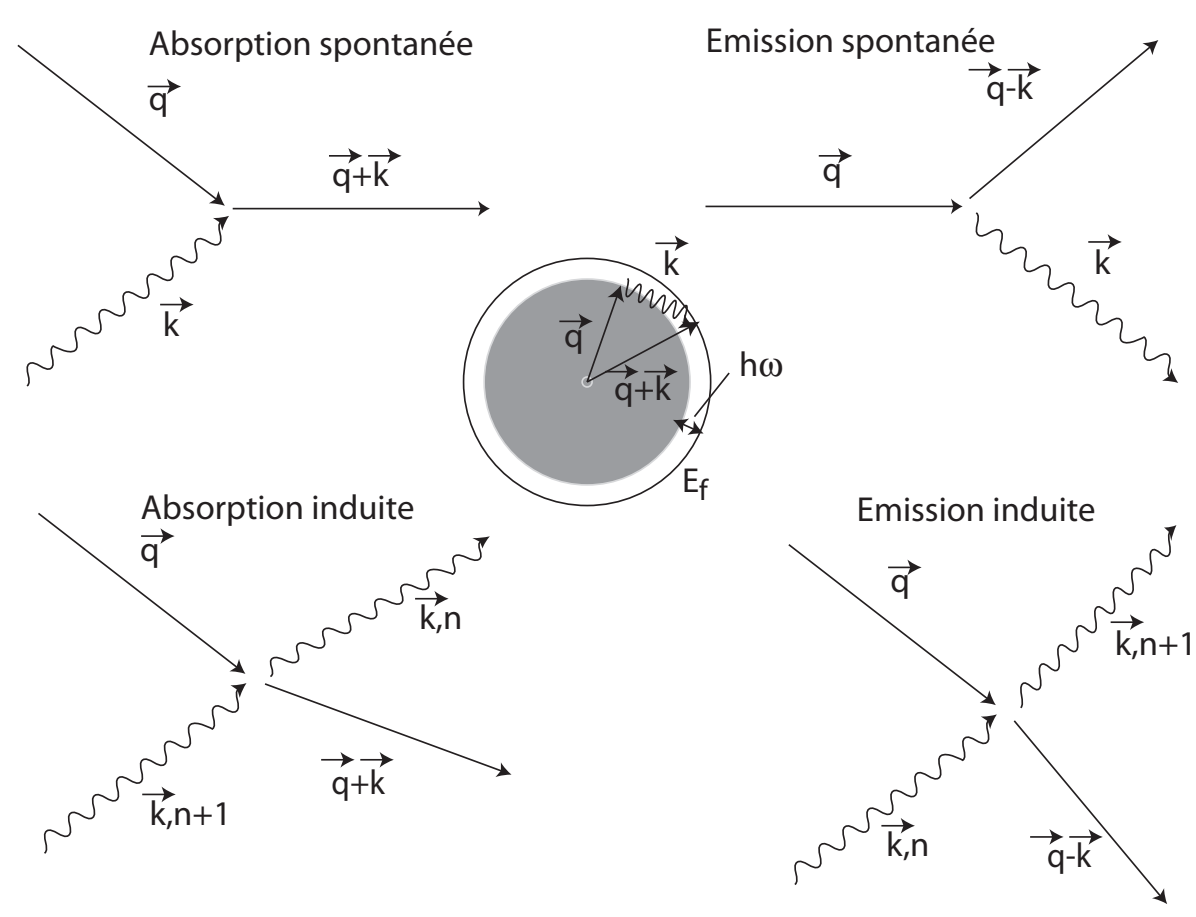

Figure 53. Illustration graphique des processus contribuant à l'interaction entre électrons et phonons. Les électrons sont représentés par les lignes droites et les phonons par des lignes ondulées. Lors des événements spontanés, les états phononiques passent de l'état fondamental à un état excité (emission) ou d'un état excité à l'état fondamental (absorption). Les événements induits sont caractérisés par le passage d'un état phononique du niveau $n$ au niveau $n+1$ (émission) ou du niveau $n+1$ au niveau $n$ (absorption). Le moment cristallin est conservé par ces processus. La figure du milieu démontre que ce sont les électrons dans une couche énergétique d'une épaisseur de $\hbar \omega$ autour de la surface de Fermi qui sont concernés.

$\mathbf{c}_{m, \sigma}^{+}(\vec{k}+\vec{q})$ est l'opérateur de création d'un électron de la bande $m$, de spin $\sigma$ et de vecteur d'onde $\vec{k}+\vec{q} \cdot \mathbf{a}_{j}(\vec{k})$ est l'opérateur d'annihilation pour un phonon de vecteur d'onde $\vec{k} \cdot \mathbf{c}_{m, \sigma}(\vec{q})$ est l'opérateur d'annihilation pour un électron de spin $\sigma$ et de vecteur d'onde $\vec{q}$. Les termes de la forme

$$
\mathbf{c}_{m, \sigma}^{+}(\vec{k}+\vec{q}) \mathbf{c}_{n, \sigma^{\prime}}(\vec{q}) \mathbf{a}_{j}(\vec{k})
$$

décrivent donc l'absorption d'un phonon de la branche $j$ et de vecteur d'onde $\vec{k}$ par l'électron de la bande $n$ avec le spin $\sigma^{\prime}$ et de vecteur d'onde $\vec{q}$. Après l'absorption du phonon, l'électron passe dans la bande $m$ et porte un spin $\sigma$ (voir diagramme de la figure 53). La probabilité de cette transition est régie par l'elément de matrice

$$
g\left(\vec{q}, m, n, \sigma, \sigma^{\prime} \mid \vec{k}, j\right) \propto\left\langle\vec{q}, m, \sigma\left|\Delta V_{j, \vec{k}}(\vec{r})\right| \vec{q}+\vec{k}, n, \sigma^{\prime}\right\rangle .
$$

$\Delta V_{j, \vec{k}}(\vec{r})$ désigne le changement dans le potentiel effectif des électrons (par exemple de type KohnSham, si nous employons la théorie de la fonctionnelle de la densité) induit par le mouvement ionique associé au mode $(j, \vec{k})$. Dans la même veine, les termes de la forme

$$
\mathbf{c}_{m, \sigma}^{+}(\vec{k}+\vec{q}) \mathbf{c}_{n, \sigma^{\prime}}(\vec{q}) \mathbf{a}_{j}^{+}(-\vec{k})
$$

sont l'expression mathématique de l'émission de phonons par les électrons. L'Hamiltonien d'interaction respecte par construction la conservation du moment cristallin, que nous avons déjà invoquée lors de la discussion des processus anharmoniques. La disymétrie entre le nombre d'opérateurs électroniques et phononiques dans l'expression 4.63 traduit le fait que le nombre d'électrons est conservé tandis que les 
phonons peuvent être créés ou annihilés. Dans le langage des quasi-particules on pourra aussi parler de la création simultanée d'un électron et d'un trou dans le lac de Fermi.

Les fonctions $g\left(\vec{q}, m, n, \sigma, \sigma^{\prime} \mid \vec{k}, j\right)$ sont les éléments de couplage électron-phonon. Elles décrivent l'interaction entre les deux sous-systèmes au-delà du simple potentiel électronique dont l'influence est déjà incorporée dans les états des phonons. C'est un point important que nous voudrions souligner. Si on étudie les phonons d'un matériau, on sonde déjà principalement le système électronique parce que c'est ce dernier qui détermine les forces d'interaction. Dans l'approximation du premier ordre, les élements de matrice sont determinés par les dérivées du potentiel ressenti par les électrons par rapport aux positions ioniques $\vec{R}(\vec{l}, \kappa)$. Pour un mode harmonique $j$ avec vecteur propre $\vec{e}_{j}(\kappa)$ nous obtenons

$$
\left\langle\vec{q}, m, \sigma\left|\Delta V_{j, \vec{k}}(\vec{r})\right| \vec{q}+\vec{k}, n, \sigma^{\prime}\right\rangle \propto \sum_{\vec{l}} \sum_{\kappa, \alpha} e_{j}(\kappa, \alpha) e^{i \vec{k} \cdot \vec{l}} \frac{\partial V(\vec{r})}{\partial \vec{R}_{\alpha}(\vec{l}, \kappa)} .
$$

Il est évident que le couplage dépendra de la symétrie des phonons et que les états électroniques réagiront de manière spécifique à la perturbation. Les phonons, qui modifieront considérablement le potentiel effectif électronique, auront une influence forte sur le paysage des électrons et faciliteront le passage entre bandes. Ces passages sont -par définition- interdits dans la structure non-perturbée. Les phonons peuvent être considérés comme des "pompes", qui transfèrent dynamiquement les électrons d'une région de la surface de Fermi vers une autre. Dans la théorie de la fonctionnelle de la densité, les constantes de couplage peuvent être calculées à partir de la structure de bande en déterminant le changement du potentiel de Kohn-Sham résultant du déplacement rigide des ions selon les vecteurs propres des phonons ("frozen phonon method"). Le couplage électron-phonon est responsable de la résistivité des métaux à haute température. C'est l'occupation des phonons qui, en augmentant linéairement avec la température, est responsable de la dépendence linéaire de la résistivité en $T$. A basse température, nous observons le phénomène inverse. Le couplage peut induire la supraconductivité. La théorie de la supraconductivité est trop complexe pour être traitée ici. Nous nous limitons à quelques descriptions sommaires, et renvoyons le lecteur au chapitre (S. Pailhès) consacré entièrement à ce sujet dans cet ouvrage. Sans pouvoir en donner la dérivation mathématique, nous remarquons qu'il est possible d'éliminer les fonctions d'onde des phonons et de décrire l'interaction instantanée entre électrons par l'Hamiltonien

$$
\begin{aligned}
\mathbf{H}_{\mathrm{el}} & =\sum_{\vec{q}} \sum_{m, \sigma} \epsilon_{m, \sigma}(\vec{q})\left(\mathbf{c}_{m, \sigma}^{+}(\vec{q}) \mathbf{c}_{m, \sigma}(\vec{q})\right) \\
& -\frac{1}{2} \sum_{\vec{k}} \sum_{\vec{q} \vec{q}^{\prime}} \sum_{m, n} \sum_{\sigma \sigma^{\prime}} v\left(\vec{q}, \vec{q}^{\prime}, \vec{k}, \sigma, \sigma^{\prime}, m, n\right)\left(\mathbf{c}_{m, \sigma}^{+}(\vec{q}+\vec{k}) \mathbf{c}_{n, \sigma^{\prime}}^{+}\left(\vec{q}^{\prime}-\vec{k}\right) \mathbf{c}_{m, \sigma}(\vec{q}) \mathbf{c}_{n, \sigma^{\prime}}\left(\vec{q}^{\prime}\right)\right) .
\end{aligned}
$$

Le deuxième terme de cet Hamiltonien décrit l'échange de moment cristallin entre deux électrons par collision. La probabilité de collision est donnée par l'élément de matrice effectif $v\left(\vec{q}, \vec{q}^{\prime}, \vec{k}, \sigma, \sigma^{\prime}, m, n\right)$, qui dépend des états initiaux et finaux des électrons ainsi que du moment $\vec{k}$ échangé. La difficulté de la supraconductivité réside dans le fait qu'on ne peut pas attaquer le problème dans une théorie de perturbation. La théorie de la supraconductivité démontre que (i) l'interaction médiée par les phonons peut être attractive et (ii) qu'un bon candidat pour l'état supraconducteur est un condensat de paires de Cooper $[39,40]$. Formellement cet état peut s'écrire comme

$$
\Phi=\sum_{m=0}^{\infty} \frac{1}{m !}\left(\sum_{\vec{q}} f_{\vec{q}} \mathbf{c}^{+}(\vec{q}, \uparrow) \mathbf{c}^{+}(-\vec{q}, \downarrow)\right)^{m}|0\rangle .
$$

$f_{q}$ est la fonction de Fermi. L'opérateur $\mathbf{c}^{+}(\vec{q}, \uparrow) \mathbf{c}^{+}(-\vec{q}, \downarrow)$ crée une paire d'électrons de vecteurs et de spins opposés. Puisque ces paires forment des bosons, elles peuvent occuper le même état à plusieurs 


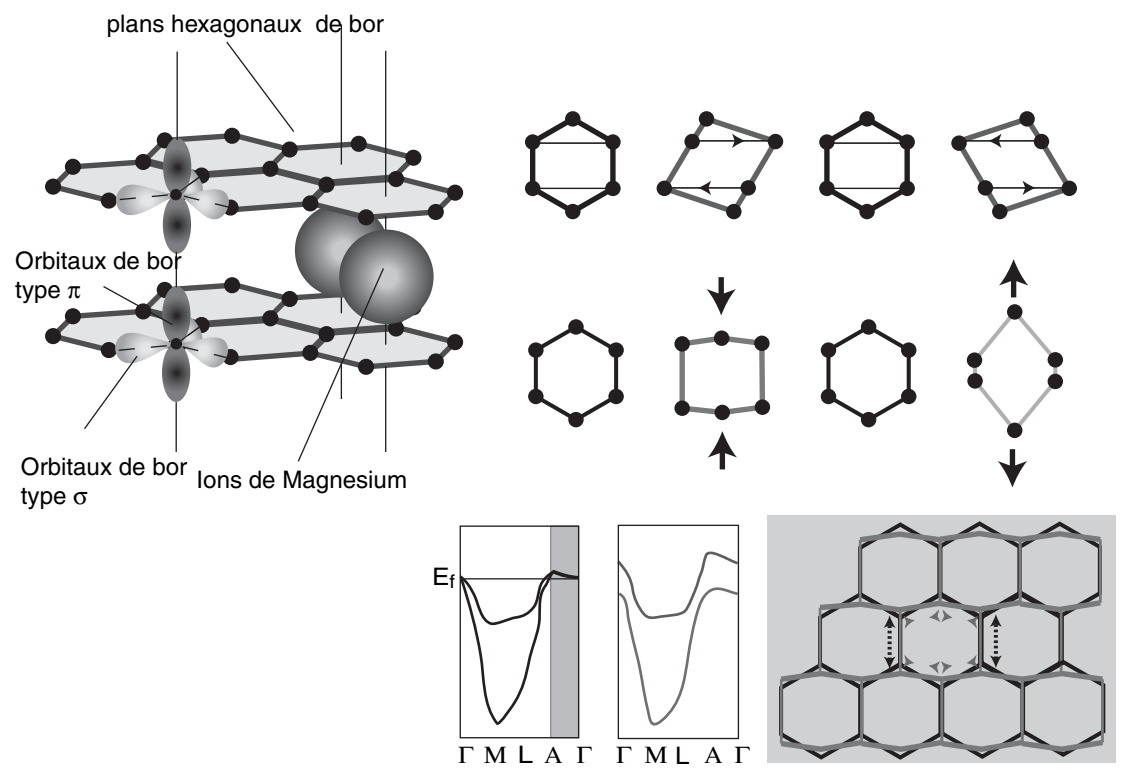

Figure 54. En haut à gauche : illustration schématique de la structure du $\mathrm{MgB}_{2}$. Il y a deux type de bandes provenant des orbitales $\sigma$ et $\pi$ des ions du bore. En haut à droite : motif de vibration du réseau des ions de bore pour deux modes au point $\Gamma$. Ces modes $\mathrm{E}_{2 g}$ sont dégénérés et, étant confinés dans le plan, montrent peu de dispersion perpendiculaire au plan, c'est-à-dire dans la direction $\Gamma-A$. En bas : une déformation des plans de bore selon le vecteurs propre de ces modes - rendant les hexagones plus rectangulaires - modifie fortement la structure des bandes de type $\sigma$, comme le démontrent les calculs [41]. C'est particulièrement bien visible dans la direction $A-\Gamma$ de l'espace réciproque ou la dégénérescence des bandes électroniques au niveau de Fermi est levée. On s'attend selon l'équation 4.67 à un fort couplage.

rendant le condensat de Bose ${ }^{94}$ possible. La forme de l'état fondamental rappelle la forme des états cohérents, que l'on avait rencontrés dans la section 3.12. Cette ressemblance n'est pas surprenante si nous considérons que l'état supraconducteur doit décrire le flux des électrons dans l'absence de résistance macroscopique.

Tous les électrons ne participent pas de la même façon au couplage. Il est évident que ceux qui seront concernés en premier lieu seront les électrons qui se trouvent proche de la surface de Fermi. Nous nous attendons à des effets particulièrement prononcés dans le cas où un vecteur $\vec{k}$ relie beaucoup d'états de la surface de Fermi entre eux. On parle de nesting de la surface de Fermi. Le nesting est un phénomème habituel dans des système de basse dimensionalité. Ces arguments ne sont pas spécifiques à la supraconductivité mais s'appliquent à tout phénomène relié au couplage électron-phonon. Un exemple typique pour un supraconducteur dont le couplage est médié par les phonons et qui montre une structure bidimensionnelle en couche est le $\mathrm{MgB}_{2}$. Il a attiré pas mal d'attention à cause de sa température de transition $T_{c} \approx 39 \mathrm{~K}$ très élevée pour un cristal aussi simple et avec un couplage conventionnel.

Jusqu'ici nous nous sommes concentrés sur les conséquences du couplage électron-phonon pour les propriétés de transport des électrons. Du côté des excitations du réseau le couplage avec les électrons produit principalement deux effets.

1. Les modes qui contribuent fortement au couplage acquièrent une largeur. Ce phénomène est analogue à celui rencontré lors de la discussion de l'anharmonicité.

\footnotetext{
${ }^{94}$ Un condensat de Bose-Einstein est un état de la matière formé de bosons caractérisé par une fraction macroscopique de particules dans l'état quantique de plus basse énergie.
} 

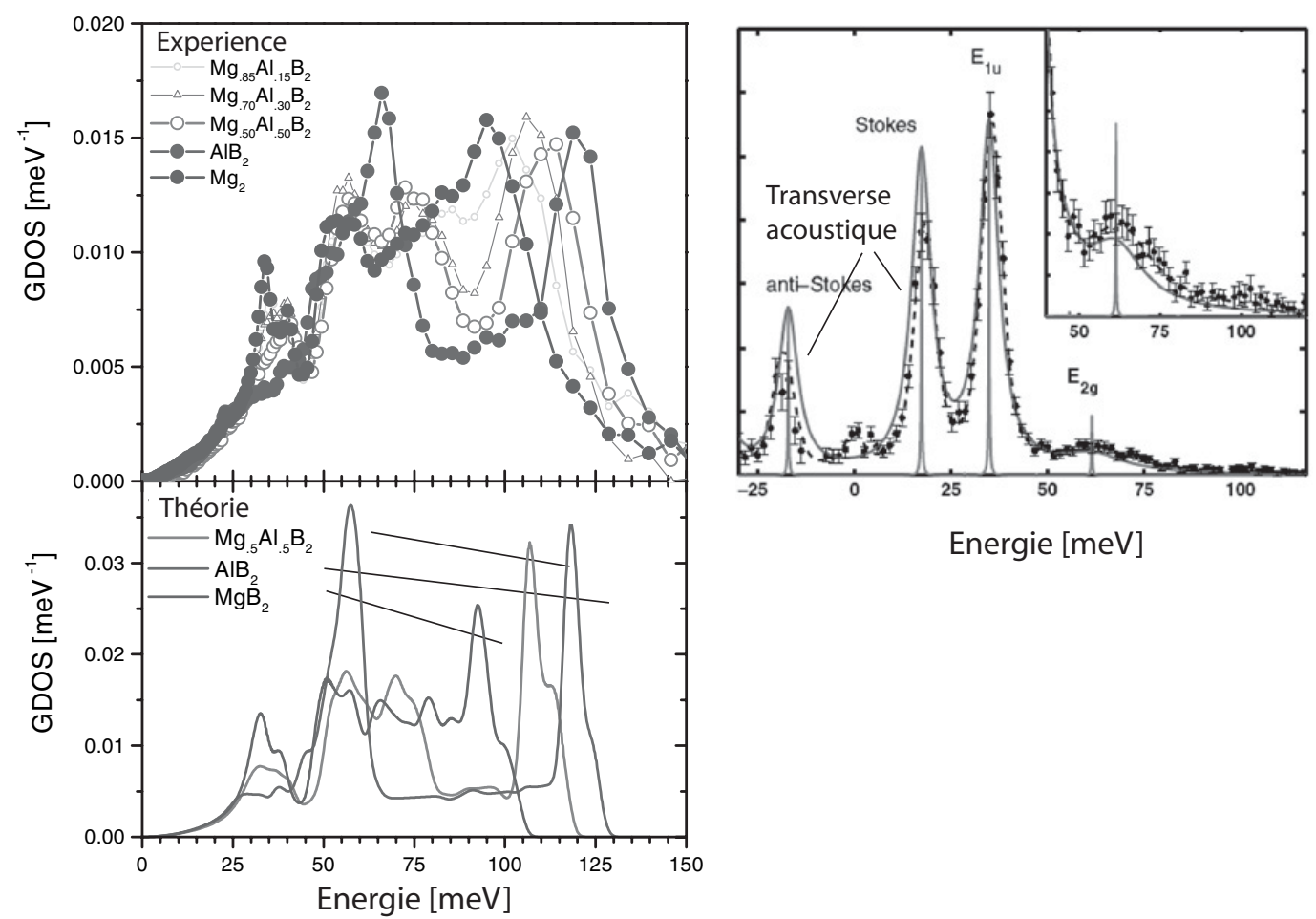

Figure 55. A gauche : densité détats des phonons pour $\mathrm{Mg}_{1-x} \mathrm{Al}_{x} \mathrm{~B}_{2}$ mesurée par diffusion inélastique des neutrons et comparaison avec un calcul DFT [42]. Le changement de l'état électronique accompagnant la substitution ionique a de fortes répercussions sur le spectre des phonons. Les modes dont les fréquences sont les plus renormalisées contribuent aussi le plus au couplage électron-phonon. Parmi eux, on trouve en particulier les modes $\mathrm{E}_{2 g}$, que nous avons déjà rencontrés (figure 54). A droite : signaux de phonons mesurés par diffusion des rayons-X sur monocristal [43]. Les données expérimentales (points et lignes interrompues) sont présentées avec des calculs intégrant l'élargissement dû au couplage électron phonon ainsi qu'avec la résolution de l'instrument (les pics fins donnent les fréquences sans couplage et sans résolution). Le signal très large correspond au mode $\mathrm{E}_{2 \mathrm{~g}}$ demontrant sa forte implication dans le couplage.

2. Les fréquences des modes impliqués dans le couplage réagissent fortement aux changements du système électronique. Expérimentalement de tels changements peuvent être induits par le dopage ou par la substitution d'ions. Il faut néanmoins bien séparer les effets dûs à un effet de masse ou à une modification de liaison, de ceux imputables purement au système électronique.

3. La transition supraconductrice modifie profondement l'état électronique. Il est donc particulièrement intéressant de scruter la région de température autour de $T_{c}$ pour déceler des modifications de la réponse phononique. On peut s'attendre à que ces effets soient particulièrement prononcés quand les énergies $\hbar \omega$ des phonons, qui sont couplés aux électrons, sont proches de $k_{\mathrm{B}} T_{c}$.

Nous montrons quelques résultat obtenus sur les phonons du $\mathrm{MgB}_{2}$ sur les figures 54 et 55 .

\section{Remerciements}

Nous voudrions remercier Efim Kats pour la correction de plusieurs formules dans la partie final de cet article ainsi que Laurence Tellier pour avoir corrigé l'orthographe. Alexander Schober à contribué la programmation des chaînes linéaires en Mathematica. H. S. voudrait dédier ce chapitre à Wolfgang Rüby, son ancien professeur de français, en espérant qu'il ne sera pas trop déçu de la qualité linguistique. 


\section{Références}

[1] N.W. Ashcroft and N.D. Mermin, Physique des Solides, EDP Science (2002).

[2] Ch. Kittel, Physique de l'état solide, Dunod (1998).

[3] M.T. Dove, Structure and Dynamics: an atomic view of materials, Oxford master series in condensed matter physics, Oxford University Press, Oxford (2003).

[4] C. Cohen-Tannoudji, B. Diu, F. Laloë, Mécanique quantique - Tome 1 et Tome 2, Hermann (2007).

[5] S.J. Blundell and K.M. Blundell, Concepts of Thermal Physics, Oxford University Press, Oxford (2006).

[6] Ch. Kittel and H. Kroemer, Thermal Physics. 2è édition, Freeman (1980).

[7] H. Schober, Neutron instrumentation in Neutron Applications in Earth, Energy and Environmental Sciences, pp. 37-104, L. Liang, R. Rinaldi and H. Schober (Eds.) Springer, New York (2008).

[8] B. Houchmandzadeh, Phys. Rev. E 66, 052902 (2002).

[9] R. Kubo, Rep. Prog. Phys. 29, 255 (1966).

[10] S.C. Johnson and T.D. Gutierrez, Am. J. Phys. 70, 227 (2002).

[11] R. Peierls, Ann. Phys. (Leibzig) 3, 1055 (1929).

[12] J.M. Ziman, Electrons and Phonons, Claredon Press, Oxford (1960).

[13] Jianjun Dong,O.F. Sankey, and Ch.W. Myles, Phys. Rev. Lett. 86, 2361 (2001).

[14] M.M. Koza and H. Schober, Vibrational Dynamics and Guest Host Coupling in Clathrate Hydrates in Neutron Applications in Earth, Energy and Environmental Sciences, pp. 351-389, L. Liang, R. Rinaldi and H. Schober (Eds.) Springer, New York (2008).

[15] J. Jensen and A.R. Mackintosh, Rare Earth Magnetism: Structures and Excitations, Clarendon Press, Oxford (1991).

[16] A.I. Krivchikov, B.Ya. Gorodilov, O.A. Korolyuk, V.G. Manzhelii, O.O. Romantsova, H. Conrad, W. Press, J.S. Tse, and D.D. Klug", Phys. Rev. B 73, 64203 (2006).

[17] J.V. Pearce, J. Bossy, H. Schober, H.R. Glyde, D.R. Daughton, and N. Mulders, Phys. Rev. Lett. 93145303 (2004).

[18] I.F. Silvera, Rev. Mod. Phys. 52, 393 (1980)

[19] S. Pisana, M. Lazzeri, C. Casiraghi, K.S. Novoselov. A.K. Geim, A.C. Ferrari, and F. Mauri, Nature Materials 6, 198 (2007).

[20] A.A. Maradudin, E.W. Montroll, G.H. Weiss, and I.P. Ipatova, Theory of Lattice Dynamics in the Harmonic Approximation, Academic, New York, (1971).

[21] R.J. Bell and P. Dean, Philos. Mag. 25, 1381 (1972).

[22] Götz Eckold, Symmetry Aspects of Excitations, Phonons in the International Tables of Crystallography, Vol. D, chapter 2.1 .

[23] J.P. Elliot, P.G. Dawber, Symmetry in Physics, Vol 1 and Vol. 2, Oxford University Press, New York (1984).

[24] J. von Neumann, E. Wigner, Physik. Z. 30, 467 (1929).

[25] S. Baroni, S. de Gironcoli, A. Dal Corso, and P. Giannozzi, Rev. Mod. Phys. 73, 515 (2001).

[26] D. Strauch and B. Dorner, J. Phys.: Condens. Matter 5, 6149 (1993).

[27] H. Schober, D. Strauch, K. Nützel, B. Dorner, J. Phys.: Condens. Matter 5, 6155 (1993).

[28] X. Gonze, J.-C. Charlier, D.C. Allan, and M.P. Teter Phys. Rev. B 50, 13035 (1994).

[29] Changyol Lee and X.Gonze, Phys. Rev B 51, 8610 (1995).

[30] M. G. Tucker, D.A. Keen, and M.T. Dove, Mineralogical Magazine, 65, 489 (2001).

[31] F. Gompf, B. Renker, H. Schober, P. Adelmann, and R. Heid, Inelastic neutron scattering results on pure and doped fullerenes, J. Supercond. 7, 643 (1994).

[32] H. Schober, A. Tölle, B. Renker, R. Heid, and F. Gompf, Phys. Rev. B, 56, 5937-5950 (1997).

[33] H. Schober, B. Renker, and R. Heid, Phys. Rev. B, 60, 998-1004 (1999). 
[34] R. Mittal, S.L. Chaplot, H. Schober, and T.A. Mary, Phys. Rev. Lett. 86, 4692 (2001).

[35] K. Schmalzl, D. Strauch, and H. Schober, Phys. Rev. B 68, 144301 (2003).

[36] B. Fåk and B. Dorner, Physica B 234-236, 1107 (1997).

[37] H. Schober, H. Itoh, A. Klapproth, V. Chihaia, and W.F. Kuhs, Eur. Phys. J. E 12, 41-49 (2003).

[38] A.A. Maradudin and A.E. Fein, Phys. Rev. 128, 2589 (1962).

[39] L.N. Cooper, Phys. Rev. 104, 1189 (1956).

[40] J. Bardeen, L.N. Cooper, and J.R. Shrieffer, 108, 1175 (1957).

[41] J.M. An and W.E. Pickett, Phys. Rev. Lett. 86, 4366 (2001).

[42] B. Renker, K.B. Bohnen, R. Heid, D. Ernst, H. Schober, M. Koza, P. Adelmann, P. Schweiss, and T. Wolf, Phys. Rev. Lett. 88, 67001 (2002).

[43] A. Shukla, M. Calandra, M. d'Astuto, M. Lazzeri, F. Mauri, C. Bellin, M. Krisch, J. Karpinski, S.M. Kazakov, J. Jun, D. Daghero, and K. Parlinski, Phys. Rev. Lett. 90, 095506 (2003).

[44] W. Kohn and L.J. Sham, Phys. Rev. 140, A1133 (1965).

[45] P. Hohenberg and W. Kohn, Phys. Rev. 136, B864 (1964).

[46] Y.-J. Han and P.G. Klemens, Phys. Rev. B 48, 6033 (1993).

[47] M. Lax, P. Hu, and V. Narayanamurti, Phys. Rev. B 23, 3095 (1981).

[48] A. Khituna and K. L. Wang, Applied Physics Letters, 79, 851 (2001).

[49] Yunfeng Gu and Yunfein Chen, Phys. Rev. B, 76 (2007).

[50] J.X. Cao, X.H. Yan, Y. Xiao, and J W. Ding, Phys. Rev. B 69, 073407 (2004). 